\title{
Total synthesis of gymnocin-A
}

Takeo Sakai, Shingo Matsushita, Shogo Arakawa, Koichi Mori, Miki Tanimoto, Akihiro Tokumasu, Tatsuji Yoshida, Yuji Mori*

Faculty of Pharmacy, Meijo University, 150 Yagotoyama, Tempaku-ku, Nagoya 468-8503, Japan

Supporting Information

Table of Contents

General S 2

Synthesis of epoxysulfone 8 $\mathrm{S} 2$

Synthesis of the FGH fragment 16 (Scheme 2) S 5

Synthesis of the FGHIJKLMN epoxysulfone 23 (Scheme 3) S 12

Synthesis of gymnocin-A (1) (Scheme 4) S 25

Copies of ${ }^{1} \mathrm{H}$ and ${ }^{13} \mathrm{C}$ NMR spectra S 43 
General All air- and moisture-sensitive reactions were carried out under an argon atmosphere in dry, freshly distilled solvents under anhydrous conditions. The term "dried" refers to the drying of an organic solution over $\mathrm{MgSO}_{4}$ followed by filtration. Flash chromatography was carried out with silica gel (spherical, neutral, particle size 40-50 mm). Melting points are uncorrected. Chemical shifts are reported in ppm relative to internal TMS ( $\delta 0.00 \mathrm{ppm}$ ) or to the solvent signals $\delta 8.71 \mathrm{ppm}\left(\mathrm{C}_{5} \mathrm{D}_{5} \mathrm{~N}\right)$ for ${ }^{1} \mathrm{H} \mathrm{NMR}$ spectra, and to the solvent signals $\delta 77.0 \mathrm{ppm}\left(\mathrm{CDCl}_{3}\right)$ or $\delta 149.9 \mathrm{ppm}\left(\mathrm{C}_{5} \mathrm{D}_{5} \mathrm{~N}\right)$ for ${ }^{13} \mathrm{C}$ NMR spectra. Data are reported as follows: chemical shift, integration, multiplicity ( $\mathrm{s}=$ singlet, $\mathrm{d}=$ doublet, $\mathrm{t}=$ triplet, $\mathrm{q}=$ quartet, $\mathrm{m}=$ multiplet, $\mathrm{br}=$ broad). The high-resolution mass spectra were recorded on a magnetic sector FAB mass spectrometer or a TOF-ESI mass spectrometer.

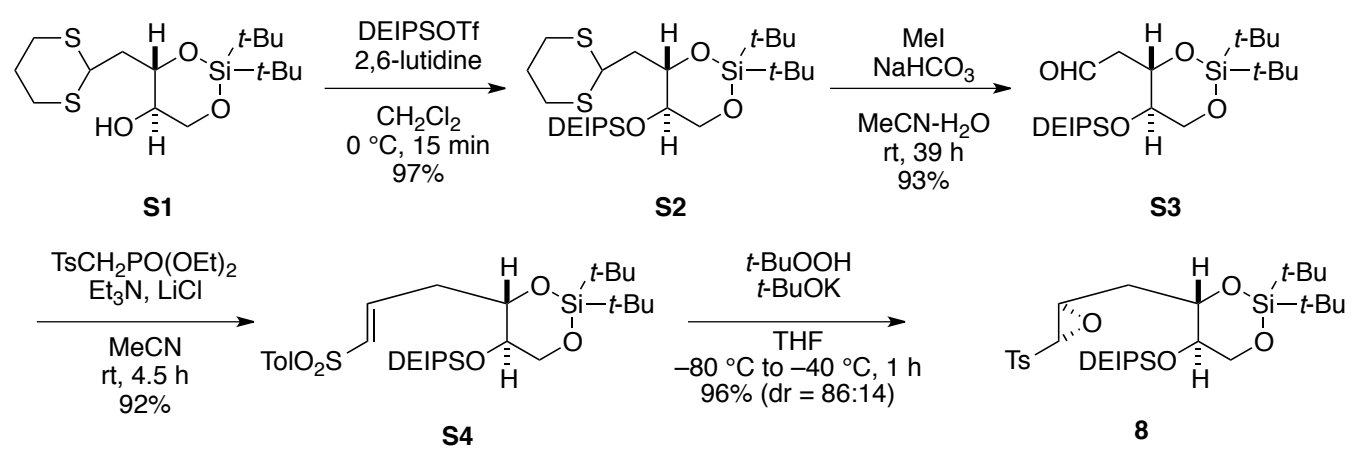

Scheme S1. Synthesis of 8

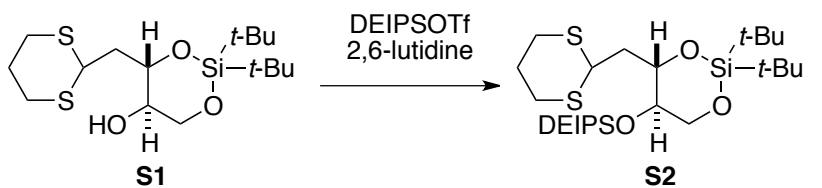

DEIPS ether S2. To a solution of alcohol S1 ${ }^{1}$ (1.04 g, $\left.2.85 \mathrm{mmol}\right)$ in $\mathrm{CH}_{2} \mathrm{Cl}_{2}(27 \mathrm{~mL})$ and 2,6-lutidine $(0.50 \mathrm{~mL}, 4.3 \mathrm{mmol})$ at $0{ }^{\circ} \mathrm{C}$ was added DEIPSOTf $(0.75 \mathrm{~mL} .3 .1$ mmol), and the reaction mixture was stirred at $0{ }^{\circ} \mathrm{C}$ for $15 \mathrm{~min}$. The reaction was quenched with saturated aqueous $\mathrm{NaHCO}_{3}$ solution, and the mixture was extracted with EtOAc. The extract was washed with water and brine, dried, and concentrated under reduced pressure. Purification by flash chromatography (4\% EtOAc in hexane) afforded

\footnotetext{
${ }^{1}$ Sakai, T.; Asano, H.; Furukawa, K.; Oshima, R.; Mori, Y. Org. Lett. 2014, 16, $2268-2271$.
} 
DEIPS ether S2 $(1.37 \mathrm{~g}, 97 \%)$ as a colorless oil. $[\alpha]^{24}-5.8\left(c 0.30, \mathrm{CHCl}_{3}\right)$; IR $\left(\mathrm{CHCl}_{3}\right)$ 1472, 1125, 1105, 1075, 1008, 944, $925 \mathrm{~cm}^{-1} ;{ }^{1} \mathrm{H} \mathrm{NMR}\left(500 \mathrm{MHz}, \mathrm{CDCl}_{3}\right) \delta$ $4.28(1 \mathrm{H}, \mathrm{dd}, J=11.0,3.5 \mathrm{~Hz}), 4.09(1 \mathrm{H}, \mathrm{ddd}, J=10.3,8.9,2.1 \mathrm{~Hz}), 4.01(1 \mathrm{H}, \mathrm{dd}, J=$ 10.3, $4.6 \mathrm{~Hz}), 3.76(1 \mathrm{H}, \mathrm{t}, J=10.3 \mathrm{~Hz}), 3.52(1 \mathrm{H}, \mathrm{ddd}, J=9.8,8.9,4.5 \mathrm{~Hz}), 2.92(1 \mathrm{H}$, ddd, $J=14.0,10.1,3.0 \mathrm{~Hz}), 2.87(1 \mathrm{H}, \mathrm{ddd}, J=14.2,5.8,3.3 \mathrm{~Hz}), 2.86(1 \mathrm{H}, \mathrm{ddd}, J=$ 14.0, 5.8, $3.3 \mathrm{~Hz}), 2.78$ (1H, ddd, $J=14.2,10.3,2.8 \mathrm{~Hz}), 2.37(1 \mathrm{H}$, ddd, $J=13.5,11.2$, $2.0 \mathrm{~Hz}), 2.11(1 \mathrm{H}, \mathrm{dtt}, J=14.2,5.8,2.9 \mathrm{~Hz}), 1.93(1 \mathrm{H}, \mathrm{dtt}, J=13.8,10.3,3.3 \mathrm{~Hz}), 1.73$ (1H, ddd, $J=13.5,10.6,3.7 \mathrm{~Hz}), 1.05(9 \mathrm{H}, \mathrm{s}), 0.98(9 \mathrm{H}, \mathrm{s}), 1.06-0.97(12 \mathrm{H}, \mathrm{m}), 0.91$ $(1 \mathrm{H}, \mathrm{m}), 0.66-0.58(4 \mathrm{H}, \mathrm{m}) ;{ }^{13} \mathrm{C} \mathrm{NMR}\left(150 \mathrm{MHz}, \mathrm{CDCl}_{3}\right) \delta 75.0,71.5,69.3,42.9,40.5$, 29.8, 29.1, 27.5, 27.1, 26.2, 22.7, 19.9, 17.24, 17.22, 12.9, 7.1, 6.9, 4.0, 3.8; HRFABMS $m / z$ calcd for $\mathrm{C}_{23} \mathrm{H}_{48} \mathrm{O}_{3} \mathrm{~S}_{2} \mathrm{Si}_{2} \mathrm{Na}\left(\mathrm{MNa}^{+}\right)$515.2481, found 515.2492.

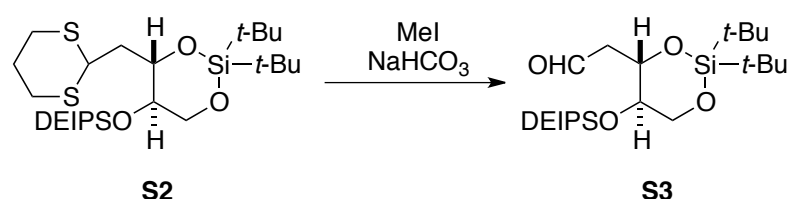

Aldehyde S3. To a solution of dithioacetal S2 (13.15 g, $44.9 \mathrm{mmol})$ in MeCN (335 mL) and water $(48 \mathrm{~mL})$ at room temperature were added $\mathrm{NaHCO}_{3}(44.82 \mathrm{~g}, 534 \mathrm{mmol})$ and MeI (49.8 mL, $803 \mathrm{mmol})$, and the suspension was stirred at room temperature for $39 \mathrm{~h}$. The reaction mixture was filtered and the residue was washed with EtOAc. The filtrate and washings are combined, concentrated to half of the volume under reduced pressure and extracted with EtOAc. The extract was washed with saturated aqueous $\mathrm{Na}_{2} \mathrm{~S}_{2} \mathrm{O}_{3}$ solution, water, brine, dried, and concentrated under reduced pressure. Purification by flash chromatography (4\% EtOAc in hexane) afforded S3 (9.98 g, 93\%) as a colorless oil. $[\alpha]^{27}$ D $-34.9\left(c 1.00, \mathrm{CHCl}_{3}\right)$; IR $\left(\mathrm{CHCl}_{3}\right) 1726,1472,1097,1011,825 \mathrm{~cm}^{-1} ;{ }^{1} \mathrm{H}$ NMR (500 MHz, $\left.\mathrm{CDCl}_{3}\right) \delta 9.85(1 \mathrm{H}, \mathrm{dd}, J=3.4,1.6 \mathrm{~Hz}), 4.38(1 \mathrm{H}, \mathrm{td}, J=9.1,3.4 \mathrm{~Hz})$, $4.06(1 \mathrm{H}, \mathrm{dd}, J=10.5,4.6 \mathrm{~Hz}), 3.81(1 \mathrm{H}, \mathrm{t}, J=10.3 \mathrm{~Hz}), 3.61(1 \mathrm{H}$, ddd, $J=9.9,8.9$, $4.6 \mathrm{~Hz}), 2.82(1 \mathrm{H}, \mathrm{ddd}, J=15.6,3.2,1.6 \mathrm{~Hz}), 2.49$ (1H, ddd, $J=15.6,9.2,3.4 \mathrm{~Hz}), 1.04$ $(9 \mathrm{H}, \mathrm{s}), 0.98(6 \mathrm{H}, \mathrm{d}, J=7.1 \mathrm{~Hz}), 0.97(6 \mathrm{H}, \mathrm{t}, J=7.8 \mathrm{~Hz}), 0.96(9 \mathrm{H}, \mathrm{s}), 0.91$ (1H, septet, $J=7.1 \mathrm{~Hz}), 0.63(4 \mathrm{H}, \mathrm{q}, J=7.8 \mathrm{~Hz}) ;{ }^{13} \mathrm{C} \mathrm{NMR}\left(150 \mathrm{MHz}, \mathrm{CDCl}_{3}\right) \delta 201.7,74.8,71.1$, 69.1, 48.8, 27.4, 26.9, 22.6, 19.8, 17.16, 17.14, 12.9, 7.0, 6.9, 4.0, 3.8; HRFABMS $m / z$ calcd for $\mathrm{C}_{20} \mathrm{H}_{43} \mathrm{O}_{4} \mathrm{Si}_{2} \mathrm{Na}\left(\mathrm{MNa}^{+}\right) 425.2519$, found 425.2504. 


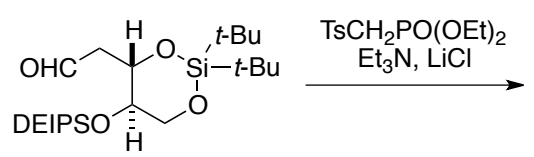

S3

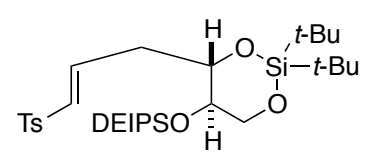

S4

Vinyl sulfone S4. To a solution of aldehyde S3 (9.98 g, $24.8 \mathrm{mmol})$, diethyl p-toluenesulfonylmethylphosphonate $(9.10 \mathrm{~g}, 29.7 \mathrm{mmol})$, and $\mathrm{LiCl}(1.26 \mathrm{~g}, 29.7$ $\mathrm{mmol})$ in $\mathrm{MeCN}(100 \mathrm{~mL})$ was added $\mathrm{Et}_{3} \mathrm{~N}(4.14 \mathrm{~mL}, 29.7 \mathrm{mmol})$, and the reaction mixture was stirred at room temperature for $4.5 \mathrm{~h}$. The reaction was quenched with saturated aqueous $\mathrm{NH}_{4} \mathrm{Cl}$ solution. The reaction mixture was extracted with EtOAc, and the extract was washed with water and brine, dried, and concentrated under reduced pressure. Purification by flash chromatography (10\% EtOAc in hexane) afforded vinyl sulfone S4 (12.65 g, 92\%) as a colorless oil. $[\alpha]^{28}-26.2\left(c 1.00, \mathrm{CHCl}_{3}\right)$; IR $\left(\mathrm{CHCl}_{3}\right)$ 1598, 1472, 1318, 1145, 1106, $825 \mathrm{~cm}^{-1} ;{ }^{1} \mathrm{H}$ NMR (500 MHz, $\left.\mathrm{CDCl}_{3}\right) \delta 7.76(2 \mathrm{H}, \mathrm{d}, J=$ $8.2 \mathrm{~Hz}), 7.30(2 \mathrm{H}, \mathrm{d}, J=8.2 \mathrm{~Hz}), 7.03(1 \mathrm{H}, \mathrm{dt}, J=14.9,7.3 \mathrm{~Hz}), 6.42(1 \mathrm{H}, \mathrm{d}, J=15.1$ $\mathrm{Hz}), 4.00(1 \mathrm{H}, \mathrm{dd}, J=10.3,4.6 \mathrm{~Hz}), 3.87(1 \mathrm{H}, \mathrm{td}, J=8.8,2.7 \mathrm{~Hz}), 3.72(1 \mathrm{H}, \mathrm{t}, J=10.3$ $\mathrm{Hz}), 3.50(1 \mathrm{H}, \mathrm{td}, J=9.5,4.7 \mathrm{~Hz}), 2.75(1 \mathrm{H}, \mathrm{dddd}, J=14.4,6.6,2.5,1.6 \mathrm{~Hz}), 2.42(3 \mathrm{H}$, s), $2.28(1 \mathrm{H}, \mathrm{dddd}, J=14.4,8.7,7.8,0.9 \mathrm{~Hz}), 0.97(9 \mathrm{H}, \mathrm{s}), 0.95(6 \mathrm{H}, \mathrm{d}, J=6.9 \mathrm{~Hz})$, $0.94(6 \mathrm{H}, \mathrm{t}, J=7.8 \mathrm{~Hz}), 0.91(9 \mathrm{H}, \mathrm{s}), 0.89(1 \mathrm{H}, \mathrm{m}), 0.61-0.55(4 \mathrm{H}, \mathrm{m}) ;{ }^{13} \mathrm{C} \mathrm{NMR}(150$ $\left.\mathrm{MHz} \mathrm{CDCl}_{3}\right) \delta 144.1,143.0,137.8,132.8,129.8,127.6,77.3,71.1,69.0,36.6,27.4$, 26.9, 22.5, 21.5, 19.8, 17.2, 17.1, 12.9, 7.0, 6.9, 4.0, 3.8; HRFABMS $m / z$ calcd for $\mathrm{C}_{28} \mathrm{H}_{50} \mathrm{O}_{5} \mathrm{SSi}_{2} \mathrm{Na}\left(\mathrm{MNa}^{+}\right)$577.2815, found 577.2802.

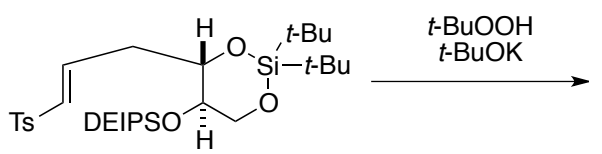

S4

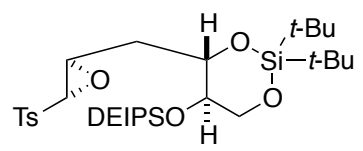

8

Epoxy sulfone 8. To a solution of $t$-BuOK (1.35 g, $12.1 \mathrm{mmol})$ in THF $(40 \mathrm{~mL})$ at $-80{ }^{\circ} \mathrm{C}$ were added $t$-BuOOH $(2.54 \mathrm{~mL}$ of a $5.5 \mathrm{M}$ solution in nonane, $14.0 \mathrm{mmol})$ and a solution of vinyl sulfone S4 $(5.17 \mathrm{~g}, 9.31 \mathrm{mmol})$ in THF $(53 \mathrm{~mL})$. The reaction mixture was stirred at $-80{ }^{\circ} \mathrm{C}$ for 10 min and warmed to $-40{ }^{\circ} \mathrm{C}$ over $1 \mathrm{~h}$, and then the reaction was quenched with saturated aqueous $\mathrm{NH}_{4} \mathrm{Cl}$ solution. The reaction mixture was extracted with EtOAc, and the extract was washed with water and brine, dried, and concentrated under reduced pressure. Purification by flash chromatography $(10 \%$ EtOAc in hexane) afforded epoxysulfone $8(5.11 \mathrm{~g}, 96 \%, 86: 14$ diastereomeric mixture by ${ }^{1} \mathrm{H}$ NMR) as a colorless oil. $[\alpha]^{27}{ }_{\mathrm{D}}-14.2\left(c 0.98, \mathrm{CHCl}_{3}\right)$; IR $\left(\mathrm{CHCl}_{3}\right) 1597,1472$, 
1328, 1154, 1108, 1010, $824 \mathrm{~cm}^{-1} ;{ }^{1} \mathrm{H}$ NMR for the major isomer $\left(500 \mathrm{MHz}, \mathrm{CDCl}_{3}\right) \delta$ $7.81(2 \mathrm{H}, \mathrm{d}, J=8.1 \mathrm{~Hz}), 7.37(2 \mathrm{H}, \mathrm{d}, J=8.1 \mathrm{~Hz}), 4.05(1 \mathrm{H}, \mathrm{d}, J=1.7 \mathrm{~Hz}), 4.00(1 \mathrm{H}, \mathrm{dd}$, $J=10.6,4.6 \mathrm{~Hz}), 3.89(1 \mathrm{H}, \mathrm{td}, J=9.1,2.8 \mathrm{~Hz}), 3.77(1 \mathrm{H}, \mathrm{ddd}, J=6.2,4.5,1.7 \mathrm{~Hz})$, $3.73(1 \mathrm{H}, \mathrm{t}, J=10.3 \mathrm{~Hz}), 3.54(1 \mathrm{H}, \mathrm{ddd}, J=9.9,9.1,4.5 \mathrm{~Hz}), 2.46(3 \mathrm{H}, \mathrm{s}), 2.09(1 \mathrm{H}$, ddd, $J=14.2,5.7,2.8 \mathrm{~Hz}), 1.88(1 \mathrm{H}$, ddd, $J=14.2,9.2,4.7 \mathrm{~Hz}), 1.03(9 \mathrm{H}, \mathrm{s}), 0.96(9 \mathrm{H}$, s), 0.96-0.92 (12H, m), $0.87(1 \mathrm{H}, \mathrm{m}), 0.62-0.56(4 \mathrm{H}, \mathrm{m}) ;{ }^{1} \mathrm{H}$ NMR for the minor isomer $\left(500 \mathrm{MHz}, \mathrm{CDCl}_{3}\right) \delta 7.81(2 \mathrm{H}, \mathrm{d}, J=8.1 \mathrm{~Hz}), 7.37(2 \mathrm{H}, \mathrm{d}, J=8.1 \mathrm{~Hz}), 4.01(1 \mathrm{H}, \mathrm{dd}, J$ $=10.6,4.6 \mathrm{~Hz}), 3.95(1 \mathrm{H}, \mathrm{ddd}, J=10.3,9.1,2.3 \mathrm{~Hz}), 3.94(1 \mathrm{H}, \mathrm{d}, J=1.7 \mathrm{~Hz}), 3.75(1 \mathrm{H}$, m), $3.73(1 \mathrm{H}, \mathrm{t}, J=10.3 \mathrm{~Hz}), 3.50(1 \mathrm{H}, \mathrm{ddd}, J=10.2,9.1,4.5 \mathrm{~Hz}), 2.46(3 \mathrm{H}, \mathrm{s}), 2.13$ $(1 \mathrm{H}, \mathrm{ddd}, J=13.9,6.8,2.0 \mathrm{~Hz}), 1.61(1 \mathrm{H}, \mathrm{ddd}, J=13.9,10.5,5.4 \mathrm{~Hz}), 1.06(9 \mathrm{H}, \mathrm{s})$, $0.99(9 \mathrm{H}, \mathrm{s}), 0.96-0.92(12 \mathrm{H}, \mathrm{m}), 0.87(1 \mathrm{H}, \mathrm{m}), 0.62-0.56(4 \mathrm{H}, \mathrm{m}) ;{ }^{13} \mathrm{C}$ NMR for the major isomer $\left(125 \mathrm{MHz}, \mathrm{CDCl}_{3}\right) \delta 145.4,133.9,129.9,128.8,75.9,70.9,69.0,68.2$, 55.6, 34.8, 27.4, 27.0, 22.5, 21.7, 19.8, 17.2, 17.1, 12.9, 7.0, 6.9, 3.9, 3.7; ${ }^{13} \mathrm{C}$ NMR for the minor isomer $\left(125 \mathrm{MHz}, \mathrm{CDCl}_{3}\right) \delta 145.4,133.9,129.9,128.8,75.9,71.4,69.0,68.2$, 55.5, 34.8, 27.5, 27.0, 22.6, 21.7, 19.8, 17.2, 17.1, 12.9, 7.0, 6.9, 3.9, 3.7; HRFABMS $m / z$ calcd for $\mathrm{C}_{28} \mathrm{H}_{50} \mathrm{O}_{6} \mathrm{SSi}_{2} \mathrm{Na}\left(\mathrm{MNa}^{+}\right)$593.2764, found 593.2783.

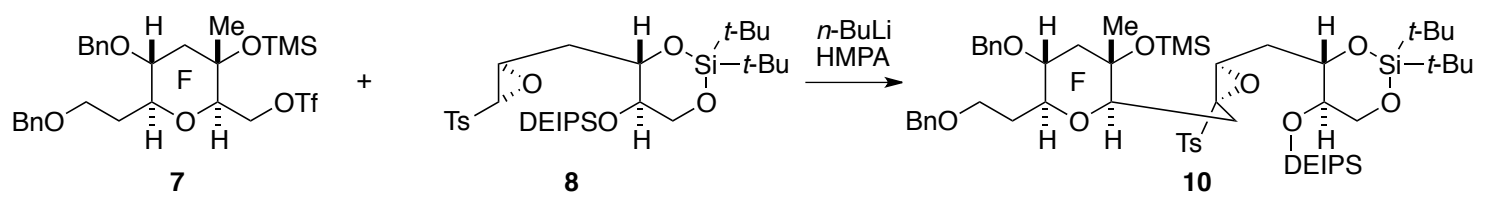

Coupling product 10. To a solution of triflate $7(683 \mathrm{mg}, 1.16 \mathrm{mmol})$ and epoxysulfone 8 (86:14 diastereomeric mixture, $1.93 \mathrm{~g}, 3.38 \mathrm{mmol})$ in THF (33 $\mathrm{mL})$ and HMPA $(3.03 \mathrm{~mL}, 17.3 \mathrm{mmol})$ at $-100{ }^{\circ} \mathrm{C}$ was added $n$-BuLi $(2.16 \mathrm{~mL}$ of a $1.55 \mathrm{M}$ solution in hexane, $3.35 \mathrm{mmol}$ ), and the reaction mixture was stirred at $-100{ }^{\circ} \mathrm{C}$ for $1 \mathrm{~h}$. The reaction was quenched with saturated aqueous $\mathrm{NH}_{4} \mathrm{Cl}$ solution. The reaction mixture was extracted with EtOAc, and the extract was washed with water and brine, dried, and concentrated under reduced pressure. Unreacted triflate 7 (61 mg, 9\%) and epoxy sulfone 8 (80:20 diastereomeric mixture, $1.22 \mathrm{~g}, 63 \%$ ) were first recovered through flash chromatography $\left(75 \% \rightarrow 100 \% \mathrm{CH}_{2} \mathrm{Cl}_{2}\right.$ in hexane), before coupling product 10 (832 mg, 71\%, 91:9 diastereomeric mixture by ${ }^{1} \mathrm{H}$ NMR) were obtained through the same chromatography $\left(6 \rightarrow 10 \%\right.$ EtOAc in hexane). Colorless oil; $[\alpha]^{27}$ -32.0 (c 1.00, $\left.\mathrm{CHCl}_{3}\right)$; IR $\left(\mathrm{CHCl}_{3}\right) 2959,2935,2863,1471,1104 \mathrm{~cm}^{-1} ;{ }^{1} \mathrm{H}$ NMR (500 $\left.\mathrm{MHz}, \mathrm{CDCl}_{3}\right) \delta 7.69(2 \mathrm{H}, \mathrm{d}, J=8.2 \mathrm{~Hz}), 7.29-7.13(12 \mathrm{H}, \mathrm{m}), 4.44$ and 4.37 (each $1 \mathrm{H}, \mathrm{d}$, 
$J=11.7 \mathrm{~Hz}), 4.42$ and $4.34($ each $1 \mathrm{H}, \mathrm{d}, J=11.5 \mathrm{~Hz}), 3.92(1 \mathrm{H}, \mathrm{dd}, J=10.4,4.7 \mathrm{~Hz})$, $3.73(1 \mathrm{H}, \mathrm{td}, J=8.8,2.7 \mathrm{~Hz}), 3.63(1 \mathrm{H}, \mathrm{dd}, J=7.8,4.1 \mathrm{~Hz}), 3.63(1 \mathrm{H}, \mathrm{t}, J=10.3 \mathrm{~Hz})$, $3.53(1 \mathrm{H}, \mathrm{td}, J=8.7,4.6 \mathrm{~Hz}), 3.49-3.42(2 \mathrm{H}, \mathrm{m}), 3.07(1 \mathrm{H}, \mathrm{d}, J=9.9 \mathrm{~Hz}), 3.01(1 \mathrm{H}, \mathrm{td}$, $J=9.6,2.1 \mathrm{~Hz}), 2.92(1 \mathrm{H}, \mathrm{ddd}, J=11.0,9.4,4.4 \mathrm{~Hz}), 2.69(1 \mathrm{H}, \mathrm{d}, J=15.4 \mathrm{~Hz}), 2.32$ $(3 \mathrm{H}, \mathrm{s}), 2.10-2.03(2 \mathrm{H}, \mathrm{m}), 1.87(1 \mathrm{H}, \mathrm{ddd}, J=14.2,7.8,2.7 \mathrm{~Hz}), 1.72(1 \mathrm{H}, \mathrm{ddd}, J=$ 14.2, 8.9, $4.3 \mathrm{~Hz}), 1.53(1 \mathrm{H}, \mathrm{dd}, J=15.3,10.3 \mathrm{~Hz}), 1.39$ (1H, dddd, $J=14.2,10.1,7.3$, $4.8 \mathrm{~Hz}), 1.35(1 \mathrm{H}, \mathrm{t}, J=11.5 \mathrm{~Hz}), 0.96(12 \mathrm{H}, \mathrm{s}), 0.88(9 \mathrm{H}, \mathrm{s}), 0.86-0.81(12 \mathrm{H}, \mathrm{m}), 0.77$ $(1 \mathrm{H}, \mathrm{m}), 0.53-0.45(4 \mathrm{H}, \mathrm{m}), 0.06(9 \mathrm{H}, \mathrm{s}) ;{ }^{13} \mathrm{C} \mathrm{NMR}\left(125 \mathrm{MHz}, \mathrm{CDCl}_{3}\right) \delta 144.8,138.9$, 138.2 , 133.6, 129.8, 129.5, 128.4, 128.2, 127.8, 127.74, 127.70, 127.3, 80.5, 78.0, 76.5, 76.4, 74.8, 73.4, 73.1, 71.3, 71.1, 69.2, 67.4, 57.6, 45.9, 32.5, 32.4, 27.5, 27.0, 23.9, 22.6, 21.7, 21.3, 19.9, 17.31, 17.26, 12.9, 7.1, 7.0, 4.0, 3.9, 2.6; HRFABMS $m / z$ calcd for $\mathrm{C}_{54} \mathrm{H}_{86} \mathrm{O}_{10} \mathrm{SSi}_{3} \mathrm{Na}\left(\mathrm{MNa}^{+}\right)$1033.5147, found 1033.5168 .

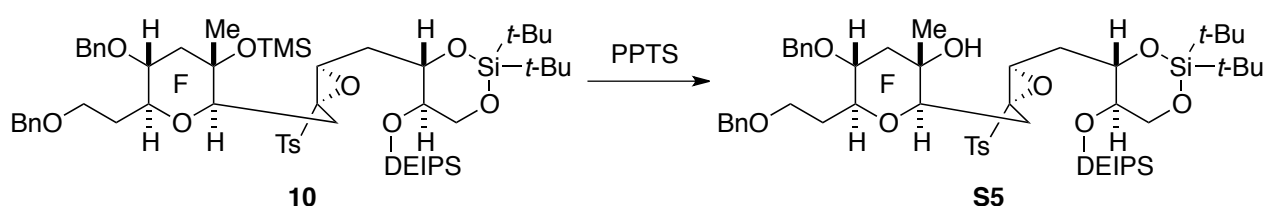

Epoxy alcohol S5. To a solution of TMS ether 10 (5.38 g, $5.32 \mathrm{mmol})$ in $\mathrm{CH}_{2} \mathrm{Cl}_{2}(53$ $\mathrm{mL}$ ) and $\mathrm{MeOH}(53 \mathrm{~mL})$ was added PPTS $(267 \mathrm{mg}, 1.06 \mathrm{mmol})$, and the reaction mixture was stirred at $0{ }^{\circ} \mathrm{C}$ for $19 \mathrm{~h}$. The reaction was quenched with $\mathrm{Et}_{3} \mathrm{~N}(2 \mathrm{~mL})$, and the reaction mixture was concentrated under reduced pressure. Purification by flash chromatography (30\% EtOAc in hexane) afforded S5 (91:9 diastereomeric mixture, $4.64 \mathrm{~g}, 93 \%)$ as a colorless amorphous solid. $[\alpha]^{24} \mathrm{D}-49.1$ (c 1.05, $\left.\mathrm{CHCl}_{3}\right)$; IR $\left(\mathrm{CHCl}_{3}\right)$ 3567, 2959, 2935, 2863, 1471, $1104 \mathrm{~cm}^{-1} ;{ }^{1} \mathrm{H}$ NMR (500 MHz, $\left.\mathrm{CDCl}_{3}\right) \delta 7.80(2 \mathrm{H}, \mathrm{d}, J$ $=8.2 \mathrm{~Hz}), 7.37-7.23(12 \mathrm{H}, \mathrm{m}), 4.56$ and 4.41 (each $1 \mathrm{H}, \mathrm{d}, J=11.6 \mathrm{~Hz}), 4.52$ and 4.46 (each 1H, d, $J=11.9 \mathrm{~Hz}), 3.99(1 \mathrm{H}, \mathrm{dd}, J=10.5,4.5 \mathrm{~Hz}), 3.77(1 \mathrm{H}, \mathrm{td}, J=9.2,2.6 \mathrm{~Hz})$, $3.71(1 \mathrm{H}, \mathrm{t}, J=10.2 \mathrm{~Hz}), 3.63(1 \mathrm{H}, \mathrm{dd}, J=8.5,4.0 \mathrm{~Hz}), 3.60(1 \mathrm{H}, \mathrm{ddd}, J=9.1,7.8,4.8$ $\mathrm{Hz}), 3.55(1 \mathrm{H}, \mathrm{ddd}, J=9.1,7.8,7.1 \mathrm{~Hz}), 3.51(1 \mathrm{H}, \mathrm{ddd}, J=10.2,9.4,4.5 \mathrm{~Hz}), 3.39(1 \mathrm{H}$, $\mathrm{d}, J=7.9 \mathrm{~Hz}), 3.25(1 \mathrm{H}, \mathrm{td}, J=9.5,2.3 \mathrm{~Hz}), 3.10(1 \mathrm{H}, \mathrm{ddd}, J=10.6,9.5,4.5 \mathrm{~Hz}), 2.75$ $(1 \mathrm{H}, \mathrm{d}, J=15.9 \mathrm{~Hz}), 2.43(3 \mathrm{H}, \mathrm{s}), 2.30(1 \mathrm{H}, \mathrm{dd}, J=11.9,4.5 \mathrm{~Hz}), 2.21(1 \mathrm{H}, \mathrm{dtd}, J=$ $13.8,7.8,2.1 \mathrm{~Hz}), 1.91(1 \mathrm{H}, \mathrm{ddd}, J=13.9,8.5,2.5 \mathrm{~Hz}), 1.82(1 \mathrm{H}, \mathrm{ddd}, J=13.9,9.6,4.0$ $\mathrm{Hz}), 1.67(1 \mathrm{H}, \mathrm{dd}, J=15.9,8.8 \mathrm{~Hz}), 1.63(1 \mathrm{H}$, br s) $1.51(1 \mathrm{H}$, dddd, $J=13.8,9.9,7.1$, $4.8 \mathrm{~Hz}), 1.38(1 \mathrm{H}, \mathrm{t}, J=11.3 \mathrm{~Hz}), 1.06(9 \mathrm{H}, \mathrm{s}), 1.05(3 \mathrm{H}, \mathrm{s}), 0.96(9 \mathrm{H}, \mathrm{s}), 0.93(3 \mathrm{H}, \mathrm{t}, J$ $=7.9 \mathrm{~Hz}), 0.93(3 \mathrm{H}, \mathrm{d}, J=7.0 \mathrm{~Hz}), 0.92(3 \mathrm{H}, \mathrm{d}, J=7.0 \mathrm{~Hz}), 0.91(3 \mathrm{H}, \mathrm{t}, J=7.9 \mathrm{~Hz})$, 
$0.86(1 \mathrm{H}, \mathrm{m}), 0.58(4 \mathrm{H}, \mathrm{q}, J=7.9 \mathrm{~Hz}) ;{ }^{13} \mathrm{C} \mathrm{NMR}\left(125 \mathrm{MHz}, \mathrm{CDCl}_{3}\right) \delta 145.4,138.8$, 138.1, 132.4, 129.9, 129.6, 128.4, 128.2, 127.76, 127.75, 127.68, 127.4, 80.8, 78.3, 76.4, 76.3, 75.0, 73.2, 71.2, 71.0, 70.6, 69.0, 67.4, 58.2, 46.0, 32.6, 32.4, 27.5, 27.0, 23.9, 22.6, 21.7, 19.95, 19.86, 17.3, 17.2, 12.8, 7.1, 7.0, 4.0, 3.8; HRFABMS $m / z$ calcd for $\mathrm{C}_{51} \mathrm{H}_{78} \mathrm{O}_{10} \mathrm{SSi}_{2} \mathrm{Na}\left(\mathrm{MNa}^{+}\right)$961.4752, found 961.4782 .

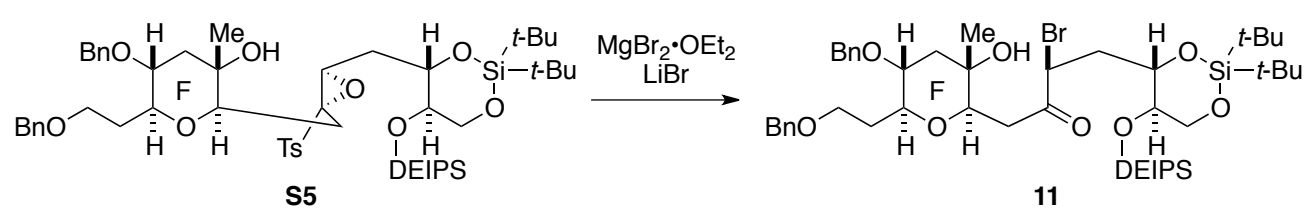

Bromoketone 11. To a solution of $\mathbf{S 5}$ (91:9 diastereomeric mixture, $1.43 \mathrm{~g}, 1.49 \mathrm{mmol}$ ) in $\mathrm{CH}_{2} \mathrm{Cl}_{2}(37 \mathrm{~mL})$ at $-15{ }^{\circ} \mathrm{C}$ were added $\mathrm{LiBr}(260 \mathrm{mg}, 2.99 \mathrm{mmol})$ and $\mathrm{MgBr}_{2} \cdot \mathrm{OEt}_{2}$ $(772 \mathrm{mg}, 2.99 \mathrm{mmol})$. The reaction mixture was warmed to $0{ }^{\circ} \mathrm{C}$ and stirred at same temperature for $3 \mathrm{~h}$, before the reaction was quenched with satd $\mathrm{NaHCO}_{3}$ solution. The resulting mixture was extracted with EtOAc, and the extract was washed with water and brine, dried, and concentrated under reduced pressure. Purification by flash chromatography $(25 \rightarrow 35 \%$ EtOAc in hexane) afforded bromoketone 11 (91:9 diastereomeric mixture, $1.28 \mathrm{~g}, 99 \%)$ as a colorless oil. $[\alpha]^{23}-63.0\left(c 1.02, \mathrm{CHCl}_{3}\right)$; IR $\left(\mathrm{CHCl}_{3}\right)$ 3587, 2960, 2935, 2862, 1717, 1472, $1102 \mathrm{~cm}^{-1} ;{ }^{1} \mathrm{H}$ NMR $\left(500 \mathrm{MHz}, \mathrm{CDCl}_{3}\right) \delta$ 7.36-7.23 (10H, m), $4.65(1 \mathrm{H}, \mathrm{dd}, J=10.5,3.7 \mathrm{~Hz}), 4.58$ and 4.44 (each $1 \mathrm{H}, \mathrm{d}, J=11.6$ $\mathrm{Hz}), 4.46$ and 4.44 (each 1H, d, $J=11.9 \mathrm{~Hz}), 4.05(1 \mathrm{H}, \mathrm{td}, J=9.5,2.0 \mathrm{~Hz}), 4.02(1 \mathrm{H}$, $\mathrm{dd}, J=10.5,4.8 \mathrm{~Hz}), 3.79(1 \mathrm{H}, \mathrm{t}, J=10.2 \mathrm{~Hz}), 3.74(1 \mathrm{H}, \mathrm{dd}, J=6.4,5.7 \mathrm{~Hz})$, $3.62-3.52(3 \mathrm{H}, \mathrm{m}), 3.38(1 \mathrm{H}, \mathrm{td}, J=9.2,2.6 \mathrm{~Hz}), 3.18(1 \mathrm{H}, \mathrm{ddd}, J=10.8,9.3,4.5 \mathrm{~Hz})$, $2.87(1 \mathrm{H}, \mathrm{d}, J=5.7 \mathrm{~Hz}), 2.87(1 \mathrm{H}, \mathrm{d}, J=6.4 \mathrm{~Hz}), 2.46(1 \mathrm{H}, \mathrm{ddd}, J=14.4,10.6,2.0 \mathrm{~Hz})$, $2.32(1 \mathrm{H}, \mathrm{dd}, J=11.9,4.5 \mathrm{~Hz}), 2.22(1 \mathrm{H}, \mathrm{dtd}, J=13.9,7.7,2.7 \mathrm{~Hz}), 1.88(1 \mathrm{H}, \mathrm{ddd}, J=$ 14.4, 10.4, $3.5 \mathrm{~Hz}), 1.62(1 \mathrm{H}, \mathrm{dddd}, J=13.9,9.6,7.1,5.1 \mathrm{~Hz}), 1.65(1 \mathrm{H}$, br s), $1.54(1 \mathrm{H}$, t, $J=11.5 \mathrm{~Hz}), 1.16(3 \mathrm{H}, \mathrm{s}), 1.06(9 \mathrm{H}, \mathrm{s}), 0.96(9 \mathrm{H}, \mathrm{s}), 0.97-0.95(12 \mathrm{H}, \mathrm{m}), 0.91(1 \mathrm{H}$, m), 0.65-0.60 (4H, m); ${ }^{13} \mathrm{C}$ NMR (125 MHz, $\left.\mathrm{CDCl}_{3}\right) \delta 202.0,138.6,138.0,128.4$, $128.3,127.8,127.74,127.73,127.4,79.7,78.4,76.2,75.9,73.1,71.4,71.1,70.4,69.2$, 67.0, 51.1, 45.9, 39.1, 38.2, 32.4, 27.5, 27.0, 22.7, 21.0, 19.8, 17.20, 17.16, 12.8, 7.0, 6.9, 3.9, 3.7; HRFABMS $m / z$ calcd for $\mathrm{C}_{44} \mathrm{H}_{71} \mathrm{O}_{8} \mathrm{BrSi}_{2} \mathrm{Na}\left(\mathrm{MNa}^{+}\right)$885.3769, found 885.3755 . 


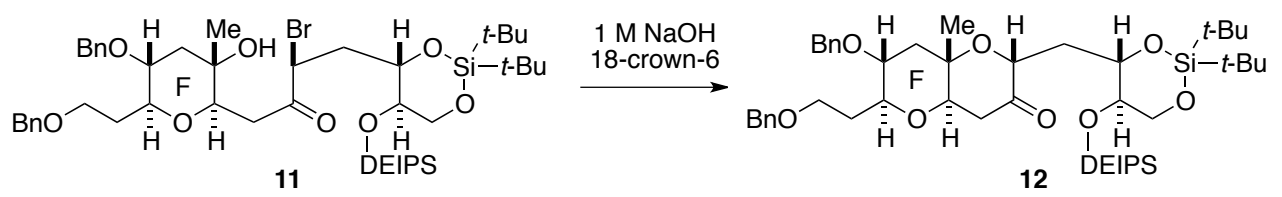

Six-membered ketone 12. To a solution of bromoketone $11(1.28 \mathrm{~g}, 1.48 \mathrm{mmol})$ in THF (148 mL) at $0{ }^{\circ} \mathrm{C}$ were added $1 \mathrm{M} \mathrm{NaOH}(4.44 \mathrm{~mL}, 4.44 \mathrm{mmol})$ and 18-crown-6 (391 $\mathrm{mg}, 1.48 \mathrm{mmol}$ ), and the reaction mixture was stirred at $0{ }^{\circ} \mathrm{C}$ for $19 \mathrm{~h}$. The reaction was quenched with saturated aqueous $\mathrm{NH}_{4} \mathrm{Cl}$ solution. The reaction mixture was extracted with EtOAc/hexane (1:1), and the extract was washed with water and brine, dried, and concentrated under reduced pressure. Purification by flash chromatography (15\% EtOAc in hexane) afforded ketone 12 (852 mg, 74\%, single isomer by ${ }^{1} \mathrm{H}$ NMR) as a colorless oil. $[\alpha]^{26}-59.3\left(c\right.$ 1.00, $\left.\mathrm{CHCl}_{3}\right)$; IR $\left(\mathrm{CHCl}_{3}\right) 2960,2936,2863,1718$, 1471, $1099 \mathrm{~cm}^{-1} ;{ }^{1} \mathrm{H}$ NMR (600 MHz, $\left.\mathrm{CDCl}_{3}\right) \delta 7.37-7.25(10 \mathrm{H}, \mathrm{m}), 4.63$ and 4.47 (each $1 \mathrm{H}, \mathrm{d}, J=11.4 \mathrm{~Hz}), 4.50$ and 4.48 (each $1 \mathrm{H}, \mathrm{d}, J=11.9 \mathrm{~Hz}), 4.15(1 \mathrm{H}, \mathrm{d}, J=6.6$ $\mathrm{Hz}), 4.12(1 \mathrm{H}, \mathrm{ddd}, J=10.8,8.8,2.6 \mathrm{~Hz}), 3.96(1 \mathrm{H}, \mathrm{dd}, J=10.5,4.4 \mathrm{~Hz}), 3.81(1 \mathrm{H}, \mathrm{dd}$, $J=11.9,6.4 \mathrm{~Hz}), 3.73(1 \mathrm{H}, \mathrm{t}, J=10.3 \mathrm{~Hz}), 3.61(1 \mathrm{H}, \mathrm{ddd}, J=9.2,7.7,5.1 \mathrm{~Hz}), 3.57$ $(1 \mathrm{H}, \mathrm{ddd}, J=9.2,7.3,7.2 \mathrm{~Hz}), 3.52(1 \mathrm{H}, \mathrm{ddd}, J=10.1,9.1,4.4 \mathrm{~Hz}), 3.42(1 \mathrm{H}, \mathrm{td}, J=$ 9.2, $2.6 \mathrm{~Hz}), 3.35(1 \mathrm{H}, \mathrm{ddd}, J=11.0,9.5,4.7 \mathrm{~Hz}), 2.75(1 \mathrm{H}, \mathrm{ddd}, J=18.5,6.4,1.1 \mathrm{~Hz})$, $2.41(1 \mathrm{H}, \mathrm{dd}, J=11.8,4.5 \mathrm{~Hz}), 2.33(1 \mathrm{H}, \mathrm{ddd}, J=13.9,6.8,2.6 \mathrm{~Hz}), 2.29(1 \mathrm{H}, \mathrm{dd}, J=$ 18.5, 12.1 Hz), 2.28 (1H, dddd, $J=14.1,7.7,7.3,2.6 \mathrm{~Hz}), 2.12$ (1H, ddd, $J=13.8,10.9$, $2.0 \mathrm{~Hz}), 1.72(1 \mathrm{H}$, dddd, $J=14.1,8.8,6.8,5.3 \mathrm{~Hz}), 1.63(1 \mathrm{H}, \mathrm{t}, J=11.5 \mathrm{~Hz}), 1.20(3 \mathrm{H}$, s), $1.00(9 \mathrm{H}, \mathrm{s}), 0.99-0.95(12 \mathrm{H}, \mathrm{m}), 0.94(9 \mathrm{H}, \mathrm{s}), 0.89(1 \mathrm{H}, \mathrm{m}), 0.65-0.60(4 \mathrm{H}, \mathrm{m}) ;{ }^{13} \mathrm{C}$ NMR $\left(150 \mathrm{MHz}, \mathrm{CDCl}_{3}\right) \delta 208.7,138.5,137.9,128.4,128.3,128.0,127.8,127.6$, 127.5, 78.6, 75.9, 75.7, 74.9, 74.0, 72.9, 71.83, 71.76, 71.1, 68.9, 66.8, 43.0, 40.1, 37.3, 32.2, 27.4, 26.9, 22.6, 20.0, 17.3, 17.2, 15.4, 12.9, 7.04, 6.97, 4.0, 3.8; HRFABMS m/z calcd for $\mathrm{C}_{44} \mathrm{H}_{70} \mathrm{O}_{8} \mathrm{Si}_{2} \mathrm{Na}\left(\mathrm{MNa}^{+}\right)$805.4507, found 805.4515.

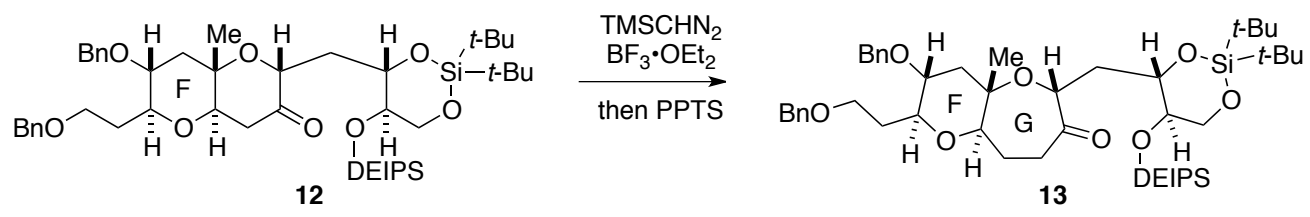

Seven-membered ketone 13. To a suspension of the six-membered ketone 12 (1.76 g, $2.25 \mathrm{mmol})$ and $\mathrm{MS} 4 \AA\left(11.3 \mathrm{~g}, 50 \mathrm{mg}\right.$ per $1 \mathrm{~mL}$ of solvent) in $\mathrm{CH}_{2} \mathrm{Cl}_{2}(225 \mathrm{~mL})$ at $-80{ }^{\circ} \mathrm{C}$ were added $\mathrm{BF}_{3} \cdot \mathrm{OEt}_{2}(1.45 \mathrm{~mL}, 11.3 \mathrm{mmol})$ and $\mathrm{TMSCH}_{2} \mathrm{~N}_{2}(5.65 \mathrm{~mL}$ of a 2.0 
$\mathrm{M}$ solution in hexane, $11.3 \mathrm{mmol}$ ), and the reaction mixture was stirred at $-80{ }^{\circ} \mathrm{C}$ for 2 h. The reaction was quenched with saturated aqueous $\mathrm{NaHCO}_{3}$ solution, and the reaction mixture was extracted with EtOAc. The extract was washed with water and brine, dried, and concentrated under reduced pressure to give the crude product $(2.12 \mathrm{~g})$. A mixture of the crude product and PPTS $(613 \mathrm{mg}, 2.44 \mathrm{mmol})$ in $\mathrm{CH}_{2} \mathrm{Cl}_{2}(72 \mathrm{~mL})$ and $\mathrm{MeOH}(72 \mathrm{~mL})$ was stirred at room temperature for $20.5 \mathrm{~h}$. The reaction was quenched with $\mathrm{Et}_{3} \mathrm{~N}(3 \mathrm{~mL})$, and the reaction mixture was concentrated under reduced pressure. Purification by flash chromatography (15\% EtOAc in hexane) afforded ketone 13 (1.62 g, 91\%) as a colorless oil. $[\alpha]^{24}-23.7$ (c 1.19, $\left.\mathrm{CHCl}_{3}\right)$; IR $\left(\mathrm{CHCl}_{3}\right)$ 2960, 2936, 2863, 1708, 1472, $1096 \mathrm{~cm}^{-1} ;{ }^{1} \mathrm{H}$ NMR (600 MHz, $\left.\mathrm{CDCl}_{3}\right) \delta 7.36-7.25(10 \mathrm{H}, \mathrm{m}), 4.61$ and 4.43 (each 1H, d, $J=11.4 \mathrm{~Hz}$ ), 4.54 and 4.45 (each 1H, d, $J=11.9 \mathrm{~Hz}), 4.19(1 \mathrm{H}, \mathrm{dd}, J$ $=7.5,3.5 \mathrm{~Hz}), 4.03(1 \mathrm{H}, \mathrm{ddd}, J=9.9,8.9,2.0 \mathrm{~Hz}), 3.98(1 \mathrm{H}, \mathrm{dd}, J=10.5,4.5 \mathrm{~Hz}), 3.75$ $(1 \mathrm{H}, \mathrm{t}, J=10.4 \mathrm{~Hz}), 3.61(2 \mathrm{H}, \mathrm{dd}, J=7.5,6.0 \mathrm{~Hz}), 3.50(1 \mathrm{H}, \mathrm{ddd}, J=9.9,9.0,4.5 \mathrm{~Hz})$, $3.40(1 \mathrm{H}, \mathrm{td}, J=9.3,2.5 \mathrm{~Hz}), 3.32(1 \mathrm{H}, \mathrm{dd}, J=11.6,4.0 \mathrm{~Hz}), 3.26(1 \mathrm{H}, \mathrm{ddd}, J=11.0$, 9.4, 4.9 Hz), 2.95 (1H, ddd, $J=14.1,12.0,2.6 \mathrm{~Hz}), 2.32(1 \mathrm{H}, \mathrm{ddd}, J=12.1,7.2,1.6 \mathrm{~Hz})$, $2.29(1 \mathrm{H}, \mathrm{dd}, J=12.1,4.8 \mathrm{~Hz}), 2.25(1 \mathrm{H}, \mathrm{dtd}, J=14.3,7.7,2.6 \mathrm{~Hz}), 2.19(1 \mathrm{H}, \mathrm{ddd}, J=$ 13.8, 7.5, $2.2 \mathrm{~Hz}), 1.85(1 \mathrm{H}, \mathrm{ddd}, J=13.9,10.1,3.5 \mathrm{~Hz}), 1.80(1 \mathrm{H}$, ddd, $J=14.1,6.6$, 4.0, 2.6 Hz), $1.69(1 \mathrm{H}, \mathrm{t}, J=11.6 \mathrm{~Hz}), 1.64(1 \mathrm{H}, \mathrm{ddt}, J=14.4,8.9,5.6 \mathrm{~Hz}), 1.46(1 \mathrm{H}$, tdd, $J=13.7,12.1,1.3 \mathrm{~Hz}), 1.09(3 \mathrm{H}, \mathrm{s}), 1.06(9 \mathrm{H}, \mathrm{s}), 0.98(3 \mathrm{H}, \mathrm{d}, J=7.1 \mathrm{~Hz}), 0.98(3 \mathrm{H}$, d, $J=7.0 \mathrm{~Hz}), 0.98(3 \mathrm{H}, \mathrm{t}, J=7.9 \mathrm{~Hz}), 0.97(3 \mathrm{H}, \mathrm{t}, J=7.9 \mathrm{~Hz}), 0.94(9 \mathrm{H}, \mathrm{s}), 0.91(1 \mathrm{H}$, m), 0.66-0.60 (4H, m); ${ }^{13} \mathrm{C}$ NMR (150 MHz, $\left.\mathrm{CDCl}_{3}\right) \delta 216.6,138.6,138.1,128.4$, $128.3,127.8,127.73,127.70,127.4,84.1,78.5,76.4$ (x2), 76.3, 74.7, 72.8, 71.4, 70.9, 68.9, 66.6, 44.6, 39.4, 38.1, 32.3, 27.6, 26.9, 25.3, 22.6, 19.9, 17.23, 17.21, 15.4, 12.9, 7.1, 7.0, 4.0, 3.8; HRFABMS $m / z$ calcd for $\mathrm{C}_{45} \mathrm{H}_{72} \mathrm{O}_{8} \mathrm{Si}_{2} \mathrm{Na}\left(\mathrm{MNa}^{+}\right)$819.4663, found 819.4662 .

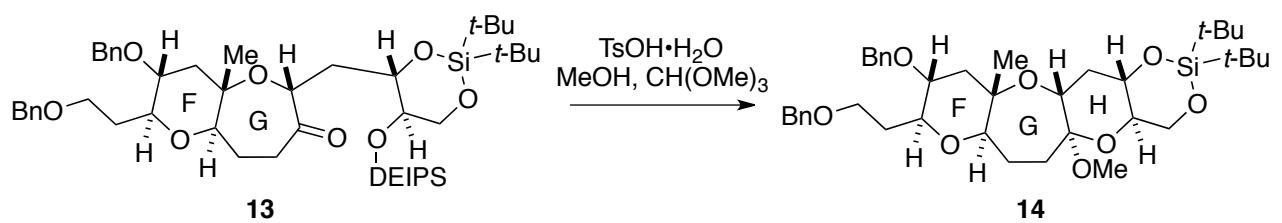

Methyl acetal 14. A mixture of ketone $13(2.22 \mathrm{~g}, 2.79 \mathrm{mmol})$ and $\mathrm{TsOH} \cdot \mathrm{H}_{2} \mathrm{O}(840 \mathrm{mg}$, $4.42 \mathrm{mmol})$ in $\mathrm{CH}_{2} \mathrm{Cl}_{2} / \mathrm{MeOH} / \mathrm{CH}(\mathrm{OMe})_{3}(10: 1: 10,56 \mathrm{~mL})$ was stirred at room temperature for $8 \mathrm{~h}$. The reaction was quenched with $\mathrm{Et}_{3} \mathrm{~N}(3 \mathrm{~mL})$, and the reaction 
mixture was concentrated under reduced pressure. Purification by flash chromatography (15\% EtOAc in hexane) afforded methyl acetal 14 (1.75 g, 94\%) as a colorless oil. $[\alpha]^{24}{ }_{\mathrm{D}}-3.8$ (c 0.33, $\left.\mathrm{CHCl}_{3}\right)$; IR $\left(\mathrm{CHCl}_{3}\right) 2935,2861,1472,1077 \mathrm{~cm}^{-1} ;{ }^{1} \mathrm{H}$ NMR (600 $\left.\mathrm{MHz}, \mathrm{CDCl}_{3}\right) \delta$ 7.35-7.25 (10H, m), 4.60 and 4.42 (each $\left.1 \mathrm{H}, \mathrm{d}, J=11.4 \mathrm{~Hz}\right), 4.52$ and 4.45 (each 1H, d, $J=12.1 \mathrm{~Hz}), 4.04(1 \mathrm{H}, \mathrm{dd}, J=10.0,4.9 \mathrm{~Hz}), 3.79(1 \mathrm{H}, \mathrm{t}, J=10.3 \mathrm{~Hz})$, $3.76(1 \mathrm{H}, \mathrm{ddd}, J=11.4,9.5,4.6 \mathrm{~Hz}), 3.62(1 \mathrm{H}, \mathrm{dd}, J=11.7,4.8 \mathrm{~Hz}), 3.59(2 \mathrm{H}, \mathrm{dd}, J=$ 7.6, 6.1 Hz), $3.48(1 \mathrm{H}, \mathrm{dd}, J=10.3,5.5 \mathrm{~Hz}), 3.42(1 \mathrm{H}, \mathrm{ddd}, J=10.5,9.5,5.0 \mathrm{~Hz}), 3.31$ $(1 \mathrm{H}, \mathrm{td}, J=9.2,2.8 \mathrm{~Hz}), 3.29(3 \mathrm{H}, \mathrm{s}), 3.20(1 \mathrm{H}, \mathrm{ddd}, J=11.2,9.3,4.5 \mathrm{~Hz}), 2.25(1 \mathrm{H}$, $\mathrm{dd}, J=12.4,4.7 \mathrm{~Hz}), 2.23(1 \mathrm{H}, \mathrm{dtd}, J=14.1,7.7,2.8 \mathrm{~Hz}), 2.12(1 \mathrm{H}, \mathrm{ddd}, J=15.5,9.1$, $4.4 \mathrm{~Hz}), 2.05(1 \mathrm{H}, \mathrm{dt}, J=12.0,4.6 \mathrm{~Hz}), 1.86-1.78(2 \mathrm{H}, \mathrm{m}), 1.68-1.59$ (3H, m), 1.53 $(1 \mathrm{H}, \mathrm{dddd}, J=14.7,10.4,8.8,4.4 \mathrm{~Hz}), 1.20(3 \mathrm{H}, \mathrm{s}), 1.03(9 \mathrm{H}, \mathrm{s}), 0.97(9 \mathrm{H}, \mathrm{s}) ;{ }^{13} \mathrm{C}$ NMR (150 MHz, $\left.\mathrm{CDCl}_{3}\right) \delta 138.7,138.3,128.34,128.29,127.8,127.6$ (x2), 127.4, 100.1, 81.0, 77.6, 76.2, 76.0, 72.7, 72.6, 72.0, 70.6, 68.9, 67.0, 66.9, 47.7, 44.5, 36.2, 32.2, 29.9, 27.4, 27.0, 24.5, 22.6, 19.9, 15.9; HRFABMS $m / z$ calcd for $\mathrm{C}_{39} \mathrm{H}_{58} \mathrm{O}_{8} \mathrm{SiNa}$ $\left(\mathrm{MNa}^{+}\right)$705.3799, found 705.3819.

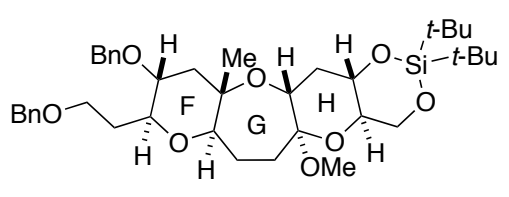

14

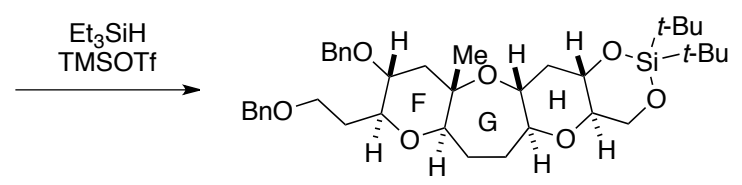

15

FGH tricyclic ether 15. To a solution of methyl acetal 14 (1.19 g,1.74 mmol) in $\mathrm{CH}_{2} \mathrm{Cl}_{2}(87 \mathrm{~mL})$ at $0{ }^{\circ} \mathrm{C}$ were added $\mathrm{Et}_{3} \mathrm{SiH}(2.8 \mathrm{~mL}, 17.4 \mathrm{mmol})$ and TMSOTf $(1.6 \mathrm{~mL}$, $8.7 \mathrm{mmol}$ ), and the reaction mixture was stirred at $0{ }^{\circ} \mathrm{C}$ for $10 \mathrm{~min}$. The reaction was quenched with saturated aqueous $\mathrm{NaHCO}_{3}$ solution, and the reaction mixture was extracted with EtOAc. The extract was washed with water and brine, dried, and concentrated under reduced pressure. Purification by flash chromatography $(15 \%$ EtOAc in hexane) afforded the tricyclic ether $15(1.01 \mathrm{~g}, 89 \%)$ as a colorless oil. $[\alpha]^{27}$ $-24.8\left(c\right.$ 1.67, $\left.\mathrm{CHCl}_{3}\right)$; IR $\left(\mathrm{CHCl}_{3}\right) 2935,2861,1473,1092 \mathrm{~cm}^{-1} ;{ }^{1} \mathrm{H}$ NMR (600 MHz, $\left.\mathrm{CDCl}_{3}\right) \delta 7.35-7.25(10 \mathrm{H}, \mathrm{m}), 4.61$ and 4.42 (each $1 \mathrm{H}, \mathrm{d}, J=11.4 \mathrm{~Hz}$ ), 4.53 and 4.44 (each $1 \mathrm{H}, \mathrm{d}, J=12.1 \mathrm{~Hz}), 4.10(1 \mathrm{H}, \mathrm{dd}, J=10.1,5.0 \mathrm{~Hz}), 3.75(1 \mathrm{H}, \mathrm{t}, J=10.3 \mathrm{~Hz})$, $3.73(1 \mathrm{H}, \mathrm{ddd}, J=11.4,9.2,4.6 \mathrm{~Hz}), 3.58(2 \mathrm{H}, \mathrm{dd}, J=7.6,6.0 \mathrm{~Hz}), 3.55(1 \mathrm{H}, \mathrm{ddd}, J=$ 11.0, 9.3, $5.0 \mathrm{~Hz}), 3.31(1 \mathrm{H}, \mathrm{ddd}, J=9.2,7.2,6.7 \mathrm{~Hz}), 3.29(1 \mathrm{H}, \mathrm{td}, J=9.2,2.6 \mathrm{~Hz})$, $3.23(1 \mathrm{H}, \mathrm{ddd}, J=11.0,9.5,4.6 \mathrm{~Hz}), 3.21(1 \mathrm{H}, \mathrm{ddd}, J=10.3,9.3,5.0 \mathrm{~Hz}), 3.08(1 \mathrm{H}, \mathrm{dd}$, 
$J=9.7,4.6 \mathrm{~Hz}), 2.29(1 \mathrm{H}, \mathrm{dt}, J=12.1,4.6 \mathrm{~Hz}), 2.28(1 \mathrm{H}, \mathrm{dd}, J=12.0,4.7 \mathrm{~Hz}), 2.23$ $(1 \mathrm{H}, \mathrm{dtd}, J=14.0,7.7,2.5 \mathrm{~Hz}), 2.11(1 \mathrm{H}, \mathrm{dq}, J=15.1,7.4 \mathrm{~Hz}), 1.76-1.65(2 \mathrm{H}, \mathrm{m})$, $1.65-1.58(2 \mathrm{H}, \mathrm{m}), 1.51(1 \mathrm{H}, \mathrm{q}, J=11.4 \mathrm{~Hz}), 1.50(1 \mathrm{H}, \mathrm{t}, J=11.5 \mathrm{~Hz}), 1.19(3 \mathrm{H}, \mathrm{s})$, $1.03(9 \mathrm{H}, \mathrm{s}), 0.97(9 \mathrm{H}, \mathrm{s}) ;{ }^{13} \mathrm{C}$ NMR $\left(150 \mathrm{MHz}, \mathrm{CDCl}_{3}\right) \delta 138.6,138.1,128.4,128.3$, 127.8, 127.7, 127.6, 127.4, 82.9, 79.9, 78.1, 76.6, 76.2, 75.3, 72.8, 72.4, 70.8, 69.0, 66.84, 66.77, 44.6, 41.0, 32.3, 29.4, 27.4, 27.0, 25.1, 22.6, 19.9, 16.2; HRFABMS m/z calcd for $\mathrm{C}_{38} \mathrm{H}_{56} \mathrm{O}_{7} \mathrm{SiNa}\left(\mathrm{MNa}^{+}\right)$675.3693, found 675.3691 .

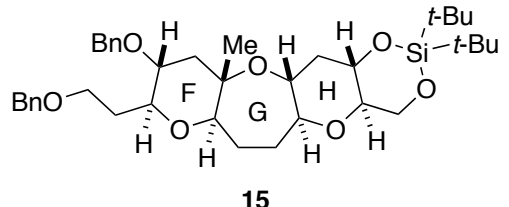

15

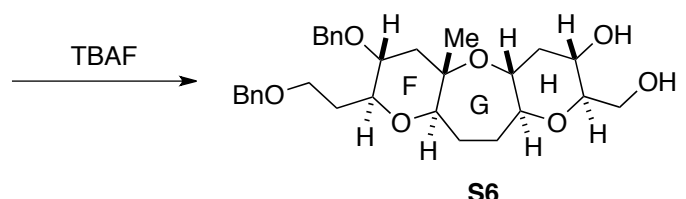

S6

FGH diol S6. To a solution of tricyclic ether 15 (366 mg, $0.560 \mathrm{mmol})$ in THF (6 mL) was added $\mathrm{Bu}_{4} \mathrm{NF}(1.51 \mathrm{~mL}$ of a $1.0 \mathrm{M}$ solution in THF, $1.51 \mathrm{mmol})$, and the reaction mixture was stirred at room temperature for $4.5 \mathrm{~h}$, before the reaction was quenched with saturated aqueous $\mathrm{NH}_{4} \mathrm{Cl}$ solution. The reaction mixture was extracted with EtOAc, and the extract was washed with brine, dried, and concentrated under reduced pressure. Purification by flash chromatography (80\% EtOAc in hexane) afforded diol S6 (276 mg, $96 \%$ ) as a colorless oil. $[\alpha]^{27}-26.1\left(c 0.30, \mathrm{CHCl}_{3}\right)$; IR $\left(\mathrm{CHCl}_{3}\right) 3600,3459,2951$, 2871, 1455, $1091 \mathrm{~cm}^{-1} ;{ }^{1} \mathrm{H}$ NMR (600 MHz, $\left.\mathrm{CDCl}_{3}\right) \delta$ 7.35-7.25 (10H, m), 4.61 and 4.42 (each $1 \mathrm{H}, \mathrm{d}, J=11.4 \mathrm{~Hz}$ ), 4.53 and 4.45 (each $1 \mathrm{H}, \mathrm{d}, J=12.1 \mathrm{~Hz}), 3.84(1 \mathrm{H}, \mathrm{dd}, J$ $=11.4,3.9 \mathrm{~Hz}), 3.74(1 \mathrm{H}, \mathrm{dd}, J=11.4,5.1 \mathrm{~Hz}), 3.61(1 \mathrm{H}, \mathrm{ddd}, J=11.0,9.5,4.8 \mathrm{~Hz})$, $3.59(2 \mathrm{H}, \mathrm{dd}, J=7.7,5.9 \mathrm{~Hz}), 3.51(1 \mathrm{H}, \mathrm{ddd}, J=10.9,9.3,4.8 \mathrm{~Hz}), 3.32-3.27(2 \mathrm{H}, \mathrm{m})$, 3.24 (1H, ddd, $J=11.0,9.3,4.8 \mathrm{~Hz}), 3.13(1 \mathrm{H}, \mathrm{ddd}, J=9.2,5.1,4.0 \mathrm{~Hz}), 3.09$ (1H, dd, $J=8.6,5.3 \mathrm{~Hz}), 2.28(1 \mathrm{H}, \mathrm{dd}, J=11.9,4.6 \mathrm{~Hz}), 2.25(1 \mathrm{H}, \mathrm{dt}, J=11.9,4.6 \mathrm{~Hz}), 2.23$ $(1 \mathrm{H}, \mathrm{dtd}, J=14.1,7.7,2.6 \mathrm{~Hz}), 2.13(1 \mathrm{H}, \mathrm{dq}, J=14.5,7.3 \mathrm{~Hz}), 2.01$ (1H, br s), 1.93 (1H, br s), 1.74-1.57 (4H, m), $1.50(1 \mathrm{H}, \mathrm{t}, J=11.6 \mathrm{~Hz}), 1.49(1 \mathrm{H}, \mathrm{q}, J=11.6 \mathrm{~Hz}), 1.19$ $(3 \mathrm{H}, \mathrm{s}) ;{ }^{13} \mathrm{C} \mathrm{NMR}\left(150 \mathrm{MHz}, \mathrm{CDCl}_{3}\right) \delta 138.6,138.1,128.4,128.3,127.8,127.69$, $127.65,127.4,83.0,80.5,79.5,78.1,76.2,75.4,72.8,70.8,68.9,66.9,66.8,63.2,44.6$, 40.9, 32.3, 29.6, 25.2, 16.2; HRFABMS $m / z$ calcd for $\mathrm{C}_{30} \mathrm{H}_{40} \mathrm{O}_{7} \mathrm{Na}\left(\mathrm{MNa}^{+}\right)$535.2672, found 535.2667. 


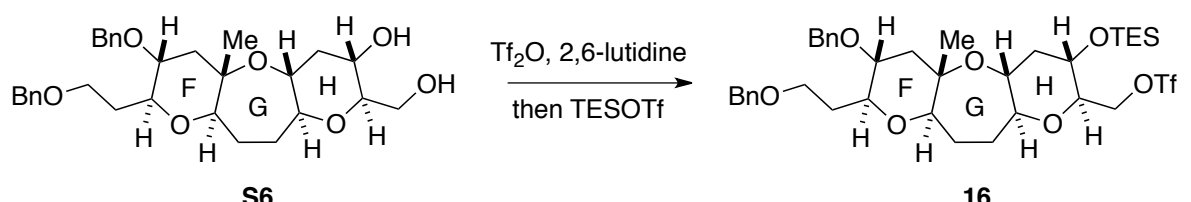

s6

16

FGH triflate 16. To a solution of diol $\mathbf{S 6}(276 \mathrm{mg}, 0.538 \mathrm{mmol})$ in 2,6-lutidine $(0.188$ $\mathrm{mL}, 1.61 \mathrm{mmol})$ and $\mathrm{CH}_{2} \mathrm{Cl}_{2}(7 \mathrm{~mL})$ at $-80{ }^{\circ} \mathrm{C}$ was added $\mathrm{Tf}_{2} \mathrm{O}(0.093 \mathrm{~mL}, 0.549$ $\mathrm{mmol})$, and the reaction mixture was stirred at $-80{ }^{\circ} \mathrm{C}$ for $40 \mathrm{~min}$. TESOTf $(0.183 \mathrm{~mL}$, $0.807 \mathrm{mmol}$ ) was then added, and the reaction mixture was stirred at $-80{ }^{\circ} \mathrm{C}$ for $30 \mathrm{~min}$. The reaction was quenched with saturated aqueous $\mathrm{NaHCO}_{3}$ solution, and the reaction mixture was extracted with EtOAc. The extract was washed with brine, dried, and concentrated under reduced pressure. Purification by flash chromatography $(10 \%$ EtOAc in hexane) afforded triflate $16(391 \mathrm{mg}, 96 \%)$ as a colorless oil. $[\alpha]^{27}+9.8(c$ 0.97, $\left.\mathrm{CHCl}_{3}\right)$; IR $\left(\mathrm{CHCl}_{3}\right) 2957,2877,1415,1145,1096,1071,956 \mathrm{~cm}^{-1} ;{ }^{1} \mathrm{H}$ NMR (600 $\left.\mathrm{MHz}, \mathrm{CDCl}_{3}\right) \delta$ 7.34-7.26 (10H, m), $4.69(1 \mathrm{H}, \mathrm{dd}, J=10.4,1.3 \mathrm{~Hz}), 4.60(1 \mathrm{H}, \mathrm{d}, J=$ $11.4 \mathrm{~Hz}), 4.53(1 \mathrm{H}, \mathrm{d}, J=11.9 \mathrm{~Hz}), 4.52(1 \mathrm{H}, \mathrm{dd}, J=10.4,5.5 \mathrm{~Hz}), 4.45(1 \mathrm{H}, \mathrm{d}, J=$ $11.9 \mathrm{~Hz}), 4.42(1 \mathrm{H}, \mathrm{d}, J=11.4 \mathrm{~Hz}), 3.59(2 \mathrm{H}, \mathrm{dd}, J=7.5,6.1 \mathrm{~Hz}), 3.56(1 \mathrm{H}, \mathrm{ddd}, J=$ 11.0, 9.4, $4.6 \mathrm{~Hz}), 3.48$ (1H, ddd, $J=10.8,9.4,4.8 \mathrm{~Hz}), 3.31-3.25(3 \mathrm{H}, \mathrm{m}), 3.23(1 \mathrm{H}$, ddd, $J=10.8,9.4,4.6 \mathrm{~Hz}), 3.08(1 \mathrm{H}, \mathrm{dd}, J=9.0,4.8 \mathrm{~Hz}), 2.28(1 \mathrm{H}, \mathrm{dd}, J=12.1,4.6$ Hz), $2.23(1 \mathrm{H}, \mathrm{dtd}, J=13.9,7.7,2.4 \mathrm{~Hz}), 2.19(1 \mathrm{H}, \mathrm{ddd}, J=12.3,4.8,4.8 \mathrm{~Hz}), 2.12$ $(1 \mathrm{H}, \mathrm{m}), 1.73-1.66(3 \mathrm{H}, \mathrm{m}), 1.62(1 \mathrm{H}, \mathrm{ddt}, J=14.2,8.7,5.9 \mathrm{~Hz}), 1.51(1 \mathrm{H}, \mathrm{q}, J=11.9$ $\mathrm{Hz}), 1.50(1 \mathrm{H}, \mathrm{t}, J=11.6 \mathrm{~Hz}), 1.20(3 \mathrm{H}, \mathrm{s}), 0.95(9 \mathrm{H}, \mathrm{t}, J=8.1 \mathrm{~Hz}), 0.61(6 \mathrm{H}, \mathrm{q}, J=8.1$ $\mathrm{Hz}) ;{ }^{13} \mathrm{C} \mathrm{NMR}\left(150 \mathrm{MHz}, \mathrm{CDCl}_{3}\right) \delta 138.6,138.1,128.4,128.3,127.8,127.68,127.66$, $127.5,118.6\left(\mathrm{q}, J_{C-F}=320.1 \mathrm{~Hz}\right), 82.8,79.7,78.5,78.1,76.2,75.6,75.5,72.8,70.8$, 68.7, 66.8, 65.9, 44.7, 41.4, 32.3, 29.2, 25.1, 16.3, 6.7, 4.9; HRFABMS $m / z$ calcd for $\mathrm{C}_{37} \mathrm{H}_{53} \mathrm{O}_{9} \mathrm{~F}_{3} \mathrm{SSiNa}\left(\mathrm{MNa}^{+}\right)$781.3029, found 781.3036.

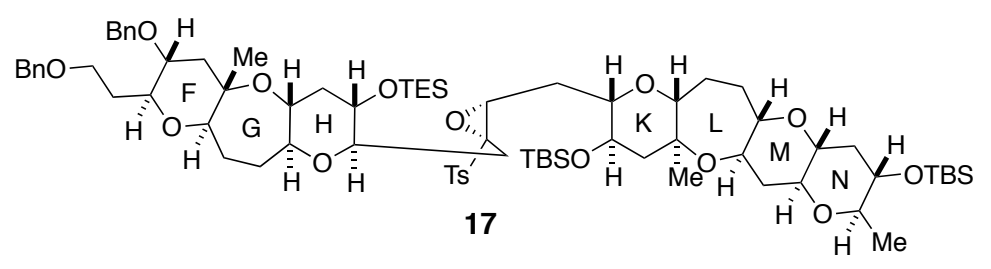

FGH-KLMN coupling product 17. To a solution of the FGH-triflate 16 (163 mg, $0.215 \mathrm{mmol})$ and the KLMN-epoxy sulfone 4 (72:28 diastereomeric mixture, $240 \mathrm{mg}$, $0.313 \mathrm{mmol})$ in THF $(4 \mathrm{~mL})$ and HMPA $(0.222 \mathrm{~mL}, 1.28 \mathrm{mmol})$ at $-100{ }^{\circ} \mathrm{C}$ was added 
$n$-BuLi $(0.192 \mathrm{~mL}$ of a $1.63 \mathrm{M}$ solution in hexane, $0.313 \mathrm{mmol})$, and the reaction mixture was stirred at $-100{ }^{\circ} \mathrm{C}$ for $0.5 \mathrm{~h}$. The reaction was quenched with saturated aqueous $\mathrm{NH}_{4} \mathrm{Cl}$ solution. The reaction mixture was extracted with EtOAc, and the extract was washed with brine, dried, and concentrated under reduced pressure. Purification by flash chromatography $\left(0 \rightarrow 10 \%\right.$ EtOAc in $\left.\mathrm{CH}_{2} \mathrm{Cl}_{2}\right)$ afforded coupling product 17 (94:6 diastereomeric mixture, $255 \mathrm{mg}, 86 \%$ ) as a colorless amorphous solid. Unreacted KLMN epoxy sulfone 4 (32:68 diastereomeric mixture, $90 \mathrm{mg}, 37 \%$ ) was recovered after flash chromatography. $[\alpha]^{27}+14.3\left(c 1.42, \mathrm{CHCl}_{3}\right) ; \mathrm{IR}\left(\mathrm{CHCl}_{3}\right) 2955$, 2877, 1463, $1078 \mathrm{~cm}^{-1} ;{ }^{1} \mathrm{H}$ NMR $\left(600 \mathrm{MHz}, \mathrm{CDCl}_{3}\right) \delta 7.81(2 \mathrm{H}, \mathrm{d}, J=8.1 \mathrm{~Hz})$, $7.36-7.26(12 \mathrm{H}, \mathrm{m}), 4.58(1 \mathrm{H}, \mathrm{d}, J=11.4 \mathrm{~Hz}), 4.52(1 \mathrm{H}, \mathrm{d}, J=11.9 \mathrm{~Hz}), 4.45(1 \mathrm{H}, \mathrm{d}, J$ $=11.9 \mathrm{~Hz}), 4.40(1 \mathrm{H}, \mathrm{d}, J=11.4 \mathrm{~Hz}), 3.86(1 \mathrm{H}, \mathrm{t}, J=6.1 \mathrm{~Hz}), 3.58(2 \mathrm{H}, \mathrm{dd}, J=6.3,7.2$ Hz), 3.54 (1H, ddd, $J=10.6,9.4,5.0 \mathrm{~Hz}), 3.44(1 \mathrm{H}, \mathrm{ddd}, J=10.8,9.3,4.9 \mathrm{~Hz})$, 3.33-3.23 (4H, m), 3.23-3.17 (2H, m), 3.16-3.09 (3H, m), 3.02-2.96 (2H, m), $2.93(1 \mathrm{H}$, ddd, $J=11.4,9.0,4.0 \mathrm{~Hz}), 2.88(1 \mathrm{H}, \mathrm{d}, J=15.4 \mathrm{~Hz}), 2.83(1 \mathrm{H}, \mathrm{t}, J=9.6 \mathrm{~Hz}), 2.69(1 \mathrm{H}$, $\mathrm{dt}, J=8.9,6.8 \mathrm{~Hz}), 2.44(3 \mathrm{H}, \mathrm{s}), 2.28-2.20(3 \mathrm{H}, \mathrm{m}), 2.20-2.10(2 \mathrm{H}, \mathrm{m}), 2.05-1.99(2 \mathrm{H}$, m), $1.96(1 \mathrm{H}, \mathrm{ddd}, J=14.6,6.7,2.9 \mathrm{~Hz}), 1.89-1.80(2 \mathrm{H}, \mathrm{m}), 1.80-1.66(3 \mathrm{H}, \mathrm{m})$, $1.65-1.54(4 \mathrm{H}, \mathrm{m}), 1.54-1.38(5 \mathrm{H}, \mathrm{m}), 1.25(1 \mathrm{H}, \mathrm{t}, J=11.4 \mathrm{~Hz}), 1.22(3 \mathrm{H}, \mathrm{d}, J=5.9$ Hz), $1.21(3 \mathrm{H}, \mathrm{s}), 1.13(3 \mathrm{H}, \mathrm{s}), 0.96(9 \mathrm{H}, \mathrm{t}, J=7.9 \mathrm{~Hz}), 0.88(9 \mathrm{H}, \mathrm{s}), 0.86(9 \mathrm{H}, \mathrm{s}), 0.60$ $(6 \mathrm{H}, \mathrm{q}, J=7.9 \mathrm{~Hz}), 0.07(6 \mathrm{H}, \mathrm{s}), 0.06(3 \mathrm{H}, \mathrm{s}), 0.04(3 \mathrm{H}, \mathrm{s}) ;{ }^{13} \mathrm{C}$ NMR $(150 \mathrm{MHz}$, $\left.\mathrm{CDCl}_{3}\right) \delta 144.8,138.6,138.1,134.4,129.54,129.46,128.34,128.28,127.8,127.65$, $127.60,127.4,83.1,83.0,80.2,79.7,79.0,78.4,78.0,77.9,76.12,76.08,75.7,75.3$, 75.2, 74.3, 72.8, 72.1, 70.7, 70.4, 69.59, 69.55, 69.2, 66.8, 57.7, 48.5, 44.7, 41.6, 39.2, $37.7,32.3,29.8,29.6,29.4,27.7,25.8,25.7,25.20,25.15,21.7,18.2,17.9,17.8,16.22$, $16.16,6.9,5.0,-4.1,-4.2,-4.7,-4.8$; HRFABMS $m / z$ calcd for $\mathrm{C}_{75} \mathrm{H}_{118} \mathrm{O}_{15} \mathrm{SSi}_{3} \mathrm{Na}$ $\left(\mathrm{MNa}^{+}\right)$1397.7397, found 1397.7371.

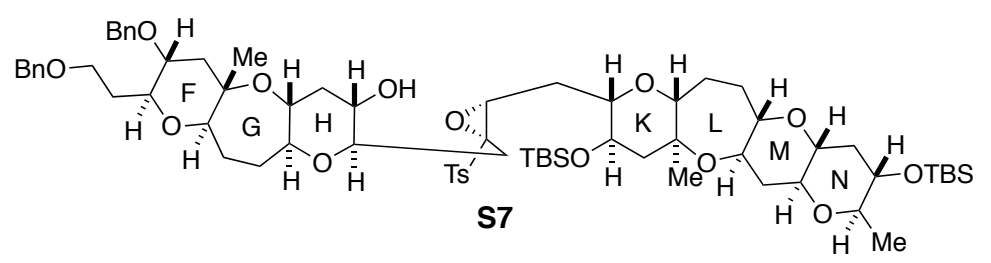

FGH-KLMN epoxy alcohol S7. To a solution of TES ether $17(255 \mathrm{mg}, 0.185 \mathrm{mmol})$ in $\mathrm{CH}_{2} \mathrm{Cl}_{2}(3.7 \mathrm{~mL})$ and $\mathrm{MeOH}(3.7 \mathrm{~mL})$ was added PPTS (50 mg, $\left.0.20 \mathrm{mmol}\right)$, and the reaction mixture was stirred at $0{ }^{\circ} \mathrm{C}$ for $19 \mathrm{~h}$. The reaction was quenched with $\mathrm{Et}_{3} \mathrm{~N}(1$ 
$\mathrm{mL}$ ), and the reaction mixture was concentrated under reduced pressure. Purification by flash chromatography (30\% EtOAc in hexane) afforded S7 (94:6 diastereomeric mixture, $231 \mathrm{mg}, 99 \%)$ as a colorless amorphous solid. $[\alpha]_{\mathrm{D}}^{27}-2.0\left(\mathrm{c} 1.22, \mathrm{CHCl}_{3}\right)$; IR $\left(\mathrm{CHCl}_{3}\right)$ 3513, 2954, 2932, 2860, $1078 \mathrm{~cm}^{-1} ;{ }^{1} \mathrm{H} \mathrm{NMR}\left(600 \mathrm{MHz}, \mathrm{CDCl}_{3}\right) \delta 7.81(2 \mathrm{H}, \mathrm{d}$, $J=8.4 \mathrm{~Hz}), 7.38-7.25(12 \mathrm{H}, \mathrm{m}), 4.60(1 \mathrm{H}, \mathrm{d}, J=11.4 \mathrm{~Hz}), 4.53(1 \mathrm{H}, \mathrm{d}, J=12.1 \mathrm{~Hz})$, $4.45(1 \mathrm{H}, \mathrm{d}, J=12.1 \mathrm{~Hz}), 4.41(1 \mathrm{H}, \mathrm{d}, J=11.4 \mathrm{~Hz}), 3.73(1 \mathrm{H}, \mathrm{dd}, J=6.8,5.5 \mathrm{~Hz}), 3.58$ $(2 \mathrm{H}, \mathrm{dd}, J=7.5,5.9 \mathrm{~Hz}), 3.53(1 \mathrm{H}$, ddd, $J=10.7,9.3,5.0 \mathrm{~Hz}), 3.41(1 \mathrm{H}$, ddd, $J=10.8$, 9.2, 4.8 Hz), $3.38(1 \mathrm{H}, \mathrm{ddd}, J=10.8,9.2,4.8 \mathrm{~Hz}), 3.33-3.16(7 \mathrm{H}, \mathrm{m}), 3.15-3.07(2 \mathrm{H}$, m), $3.02(1 \mathrm{H}, \mathrm{dd}, J=8.0,6.3 \mathrm{~Hz}), 3.00-2.97(2 \mathrm{H}, \mathrm{m}), 2.93(1 \mathrm{H}, \mathrm{ddd}, J=11.7,9.0,4.2$ $\mathrm{Hz}), 2.55(1 \mathrm{H}, \mathrm{dd}, J=15.9,3.6 \mathrm{~Hz}), 2.46(3 \mathrm{H}, \mathrm{s}), 2.28-2.10(6 \mathrm{H}, \mathrm{m}), 2.03-1.92(3 \mathrm{H}$, $\mathrm{m}), 1.88(1 \mathrm{H}, \mathrm{d}, J=7.1 \mathrm{~Hz}, \mathrm{OH}), 1.87(1 \mathrm{H}, \mathrm{dd}, J=15.9,6.8 \mathrm{~Hz}), 1.83-1.67(4 \mathrm{H}, \mathrm{m})$, $1.66-1.60(3 \mathrm{H}, \mathrm{m}), 1.57-1.37(5 \mathrm{H}, \mathrm{m}), 1.32(1 \mathrm{H}, \mathrm{t}, J=11.0 \mathrm{~Hz}), 1.22(3 \mathrm{H}, \mathrm{d}, J=6.1$ $\mathrm{Hz}), 1.19(3 \mathrm{H}, \mathrm{s}), 1.15(3 \mathrm{H}, \mathrm{s}), 0.88(9 \mathrm{H}, \mathrm{s}), 0.85(9 \mathrm{H}, \mathrm{s}), 0.071(3 \mathrm{H}, \mathrm{s}), 0.067(3 \mathrm{H}, \mathrm{s})$, $0.06(3 \mathrm{H}, \mathrm{s}), 0.03(3 \mathrm{H}, \mathrm{s}) ;{ }^{13} \mathrm{C} \mathrm{NMR}\left(150 \mathrm{MHz}, \mathrm{CDCl}_{3}\right) \delta 145.3,138.6,138.1,133.1$, $129.7,129.6,128.4,128.3,127.8,127.7,127.6,127.4,83.14,83.11,80.1,79.7,79.4$, $78.5,78.1,77.7,76.2,76.1,75.7,75.3,75.1,74.2,72.8,72.1,70.8,69.8,69.60,69.58$, 69.1, 66.8, 58.6, 48.5, 44.6, 41.6, 39.2, 37.7, 32.3, 29.9, 29.6, 29.5, 28.6, 25.8, 25.7, $25.25,25.20,21.7,18.2,17.9,17.8,16.20,16.16,-4.1,-4.2,-4.7,-4.8$; HRFABMS $m / z$ calcd for $\mathrm{C}_{69} \mathrm{H}_{104} \mathrm{O}_{15} \mathrm{SSi}_{2} \mathrm{Na}\left(\mathrm{MNa}^{+}\right)$1260.6532, found 1260.6555 .

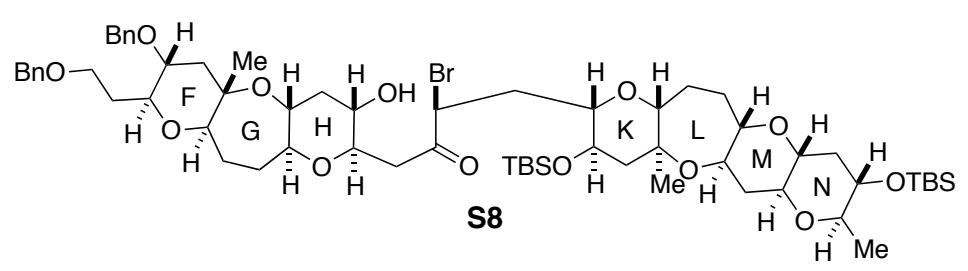

FGH-KLMN bromoketone S8. To a solution of $\mathbf{S 7}$ (94:6 diastereomeric mixture, 1.43 $\mathrm{g}, 1.49 \mathrm{mmol})$ and $\mathrm{LiBr}(31.8 \mathrm{mg}, 0.366 \mathrm{mmol})$ in $\mathrm{CH}_{2} \mathrm{Cl}_{2}(37 \mathrm{~mL})$ at $-15^{\circ} \mathrm{C}$ was added $\mathrm{MgBr}_{2} \cdot \mathrm{OEt}_{2}$ (94 mg, $0.366 \mathrm{mmol}$ ), and the mixture was stirred at $-15{ }^{\circ} \mathrm{C}$ for $1 \mathrm{~h}$. An additional amount of $\mathrm{MgBr}_{2} \cdot \mathrm{OEt}_{2}$ (94 $\mathrm{mg}, 0.366 \mathrm{mmol}$ ) was added, and then the reaction mixture was stirred at $0{ }^{\circ} \mathrm{C}$ for $6 \mathrm{~h}$, before the reaction was quenched with saturated aqueous $\mathrm{NaHCO}_{3}$ solution. The resulting mixture was extracted with EtOAc, and the extract was washed with brine, dried, and concentrated under reduced pressure. Purification by flash chromatography (30\% EtOAc in hexane) afforded bromoketone $\mathbf{S 8}$ (90:10 diastereomeric mixture, $207 \mathrm{mg}, 96 \%$ ) as a colorless amorphous solid. $[\alpha]^{28}$ 
-27.0 (c 0.97, $\left.\mathrm{CHCl}_{3}\right)$; IR $\left(\mathrm{CHCl}_{3}\right) 3513,2954,2932,2860,1716,1075 \mathrm{~cm}^{-1} ;{ }^{1} \mathrm{H} \mathrm{NMR}$ $\left(600 \mathrm{MHz}, \mathrm{CDCl}_{3}\right) \delta 7.36-7.25(10 \mathrm{H}, \mathrm{m}), 4.60(1 \mathrm{H}, \mathrm{d}, J=11.4 \mathrm{~Hz}), 4.56(1 \mathrm{H}, \mathrm{dd}, J=$ $10.3,3.9 \mathrm{~Hz}), 4.53(1 \mathrm{H}, \mathrm{d}, J=12.1 \mathrm{~Hz}), 4.45(1 \mathrm{H}, \mathrm{d}, J=12.1 \mathrm{~Hz}), 4.41(1 \mathrm{H}, \mathrm{d}, J=11.4$ $\mathrm{Hz}), 3.59(2 \mathrm{H}, \mathrm{dd}, J=7.4,6.1 \mathrm{~Hz}), 3.52(1 \mathrm{H}, \mathrm{ddd}, J=10.8,9.4,5.0 \mathrm{~Hz}), 3.50-3.45(2 \mathrm{H}$, m), $3.40(1 \mathrm{H}, \mathrm{ddd}, J=10.8,9.0,4.8 \mathrm{~Hz}), 3.36(1 \mathrm{H}, \mathrm{dddd}, J=10.8,9.0,5.8,4.8 \mathrm{~Hz})$, 3.16-3.32 (7H, m), $3.13(1 \mathrm{H}, \mathrm{dd}, J=10.4,3.8 \mathrm{~Hz}), 3.07(1 \mathrm{H}, \mathrm{t}, J=7.2 \mathrm{~Hz}), 3.06(1 \mathrm{H}$, dd, $J=16.0,4.6 \mathrm{~Hz}), 2.98$ (1H, ddd, $J=11.4,9.0,4.2 \mathrm{~Hz}), 2.92(1 \mathrm{H}, \mathrm{ddd}, J=11.4,9.0$, $4.2 \mathrm{~Hz}), 2.87(1 \mathrm{H}, \mathrm{dd}, J=16.0,6.8 \mathrm{~Hz}), 2.35(1 \mathrm{H}, \mathrm{ddd}, J=14.9,10.4,2.0 \mathrm{~Hz})$, 2.29-2.20 (4H, m), $2.16(1 \mathrm{H}, \mathrm{dt}, J=12.0,4.4 \mathrm{~Hz}), 2.14-2.05(2 \mathrm{H}, \mathrm{m}), 2.01(1 \mathrm{H}, \mathrm{dd}, J=$ 12.3, $4.8 \mathrm{~Hz}), 1.86-1.75(3 \mathrm{H}, \mathrm{m}), 1.74-1.58(6 \mathrm{H}, \mathrm{m}), 1.56(1 \mathrm{H}, \mathrm{t}, J=11.6 \mathrm{~Hz}), 1.49$ $(1 \mathrm{H}, \mathrm{t}, J=11.6 \mathrm{~Hz}), 1.44(1 \mathrm{H}, \mathrm{q}, J=11.6 \mathrm{~Hz}), 1.42(1 \mathrm{H}, \mathrm{q}, J=11.6 \mathrm{~Hz}), 1.41(1 \mathrm{H}, \mathrm{q}, J$ $=11.2 \mathrm{~Hz}), 1.21(3 \mathrm{H}, \mathrm{d}, J=6.2 \mathrm{~Hz}), 1.19(3 \mathrm{H}, \mathrm{s}), 1.17(3 \mathrm{H}, \mathrm{s}), 0.87(18 \mathrm{H}, \mathrm{s}), 0.07(3 \mathrm{H}$, s), $0.06(6 \mathrm{H}, \mathrm{s}), 0.06(3 \mathrm{H}, \mathrm{s}) ;{ }^{13} \mathrm{C} \mathrm{NMR}\left(150 \mathrm{MHz}, \mathrm{CDCl}_{3}\right) \delta 202.4,138.6,138.1,128.4$, $128.3,127.8,127.7,127.6,127.4,83.1,83.0,79.7,79.63,79.56,78.4,78.1,77.6,76.2$, 76.1, 75.7, 75.4, 75.2, 72.8, 72.1, 70.8, 70.1, 69.6, 69.4, 69.0, 66.8, 51.2, 48.6, 44.6, 42.2, 41.3, 39.2, 37.7, 35.8, 32.3, 29.6, 29.5, 25.7 (x2), 25.2, 25.1, 18.2, 17.90, 17.88, $16.24,16.22,-4.0,-4.2,-4.7,-4.8$; HRFABMS $m / z$ calcd for $\mathrm{C}_{62} \mathrm{H}_{97} \mathrm{BrO}_{13} \mathrm{Si}_{2} \mathrm{Na}$ $\left(\mathrm{MNa}^{+}\right)$1207.5549, found 1207.5549.



FGHI-KLMN six-membered ketone 18. To a solution of bromoketone S8 (203 mg, $0.171 \mathrm{mmol})$ in $\mathrm{CH}_{2} \mathrm{Cl}_{2}(3.4 \mathrm{~mL})$ was added DBU $(0.0281 \mathrm{~mL}, 0.188 \mathrm{mmol})$ at room temperature, and the reaction mixture was stirred for $2 \mathrm{~h}$. The reaction was quenched with saturated aqueous $\mathrm{NH}_{4} \mathrm{Cl}$ solution, and the mixture was extracted with EtOAc. The extract was washed with brine, dried, and concentrated. Purification by flash chromatography (30\% EtOAc in hexane) afforded cyclic ketone 18 (single diastereomer, $164 \mathrm{mg}, 87 \%)$ as a colorless amorphous solid; $[\alpha]^{25}{ }_{\mathrm{D}}-5.0\left(c \mathrm{1.11}, \mathrm{CHCl}_{3}\right)$; IR $\left(\mathrm{CHCl}_{3}\right)$ 2955, 2933, 2859, 1724, $1075 \mathrm{~cm}^{-1}$; ${ }^{1} \mathrm{H}$ NMR (600 MHz, $\left.\mathrm{CDCl}_{3}\right) \delta 7.25-7.35$ (10H, m), $4.62(1 \mathrm{H}, \mathrm{d}, J=11.4 \mathrm{~Hz}), 4.53(1 \mathrm{H}, \mathrm{d}, J=12.1 \mathrm{~Hz}), 4.45(1 \mathrm{H}, \mathrm{d}, J=12.1 \mathrm{~Hz}), 4.42(1 \mathrm{H}$, d, $J=11.4 \mathrm{~Hz}), 3.98(1 \mathrm{H}, \mathrm{dd}, J=6.0,4.5 \mathrm{~Hz}), 3.60(1 \mathrm{H}, \mathrm{ddd}, J=11.2,9.2,4.8 \mathrm{~Hz})$, 
$3.59(2 \mathrm{H}, \mathrm{dd}, J=7.3,6.1 \mathrm{~Hz}), 3.51(1 \mathrm{H}, \mathrm{ddd}, J=10.6,9.2,4.8 \mathrm{~Hz}), 3.47(1 \mathrm{H}, \mathrm{ddd}, J=$ 11.0, 9.4, 5.0 Hz), 3.16-3.35 (9H, m), $3.11(1 \mathrm{H}, \mathrm{t}, J=6.7 \mathrm{~Hz}), 3.08(1 \mathrm{H}, \mathrm{t}, J=6.7 \mathrm{~Hz})$, $2.98(1 \mathrm{H}, \mathrm{ddd}, J=11.6,9.0,4.0 \mathrm{~Hz}), 2.92(1 \mathrm{H}, \mathrm{ddd}, J=11.4,9.2,4.0 \mathrm{~Hz}), 2.88(1 \mathrm{H}, \mathrm{dd}$, $J=16.3,5.0 \mathrm{~Hz}), 2.35-2.26(4 \mathrm{H}, \mathrm{m}), 2.26-2.20(2 \mathrm{H}, \mathrm{m}), 2.19-2.11(2 \mathrm{H}, \mathrm{m}), 2.07(1 \mathrm{H}$, m), $1.96(1 \mathrm{H}, \mathrm{dd}, J=12.3,4.8 \mathrm{~Hz}), 1.77(1 \mathrm{H}, \mathrm{ddd}, J=14.3,8.8,4.2 \mathrm{~Hz}), 1.65-1.75(6 \mathrm{H}$, m), $1.62(1 \mathrm{H}, \mathrm{ddt}, J=14.2,9.0,5.9 \mathrm{~Hz}), 1.55(1 \mathrm{H}, \mathrm{q}, J=11.4 \mathrm{~Hz}), 1.52(1 \mathrm{H}, \mathrm{t}, J=11.2$ $\mathrm{Hz}), 1.50(1 \mathrm{H}, \mathrm{t}, J=11.2 \mathrm{~Hz}), 1.42(1 \mathrm{H}, \mathrm{q}, J=11.6 \mathrm{~Hz}), 1.41(1 \mathrm{H}, \mathrm{q}, J=11.2 \mathrm{~Hz}), 1.21$ $(3 \mathrm{H}, \mathrm{d}, J=5.9 \mathrm{~Hz}), 1.21(3 \mathrm{H}, \mathrm{s}), 1.19(3 \mathrm{H}, \mathrm{s}), 0.87(9 \mathrm{H}, \mathrm{s}), 0.86(9 \mathrm{H}, \mathrm{s}), 0.064(3 \mathrm{H}, \mathrm{s})$, $0.062(3 \mathrm{H}, \mathrm{s}), 0.060(3 \mathrm{H}, \mathrm{s}), 0.04(3 \mathrm{H}, \mathrm{s}) ;{ }^{13} \mathrm{C} \mathrm{NMR}\left(150 \mathrm{MHz}, \mathrm{CDCl}_{3}\right) \delta 206.2,138.6$, $138.1,128.4,128.3,127.8,127.7,127.6,127.4,83.0,82.9,79.6,79.49,79.47,78.6$, $78.4,78.1,76.2,76.1,75.7,75.5,75.4,75.3,74.7,72.7,72.0,70.8,70.4,69.8,69.1$, $66.7,48.8,44.6,44.5,39.2,37.7,37.6,32.5,32.3,29.6,29.5,25.73,25.70,25.2,25.1$, $18.1,17.89,17.85,16.3,16.2,-4.1,-4.2,-4.7,-4.8$; HRFABMS $m / z$ calcd for $\mathrm{C}_{62} \mathrm{H}_{96} \mathrm{O}_{13} \mathrm{SiNa}\left(\mathrm{MNa}^{+}\right)$1127.6287, found 1127.6270.



FGHIJKLMN nonacyclic acetal S9. A mixture of ketone 18 (164 mg, $0.148 \mathrm{mmol})$ and $\mathrm{TsOH} \cdot \mathrm{H}_{2} \mathrm{O}(85 \mathrm{mg}, 0.44 \mathrm{mmol})$ in 1,2-dichloroethane $(3 \mathrm{~mL})$ and $\mathrm{MeOH}(3 \mathrm{~mL})$ was refluxed for $1 \mathrm{~h}$. Trimethyl orthoformate $(2.0 \mathrm{~mL}, 18.3 \mathrm{mmol})$ was then added and the reaction mixture was refluxed for additional $2 \mathrm{~h}$. After cooling to room temperature, the reaction was quenched with $\mathrm{Et}_{3} \mathrm{~N}(0.5 \mathrm{~mL})$, and the reaction mixture was concentrated under reduced pressure. Purification by flash chromatography $(50 \%$ EtOAc in $\mathrm{CH}_{2} \mathrm{Cl}_{2}$ ) afforded methyl acetal $\mathbf{S 9}(120 \mathrm{mg}, 91 \%)$ as a colorless solid. Mp $250-253{ }^{\circ} \mathrm{C} ;[\alpha]_{\mathrm{D}}^{28}+29.5\left(c \mathrm{1.34}, \mathrm{CHCl}_{3}\right)$; IR $\left(\mathrm{CHCl}_{3}\right)$ 3502, 2952, 2875, 1092, 1059, $1038 \mathrm{~cm}^{-1} ;{ }^{1} \mathrm{H}$ NMR $\left(600 \mathrm{MHz}, \mathrm{CDCl}_{3}\right) \delta 7.36-7.24(10 \mathrm{H}, \mathrm{m}), 4.61(1 \mathrm{H}, \mathrm{d}, J=11.4$ $\mathrm{Hz}), 4.52(1 \mathrm{H}, \mathrm{d}, J=12.1 \mathrm{~Hz}), 4.44(1 \mathrm{H}, \mathrm{d}, J=12.1 \mathrm{~Hz}), 4.40(1 \mathrm{H}, \mathrm{d}, J=11.4 \mathrm{~Hz})$, $3.58(2 \mathrm{H}, \mathrm{dd}, J=7.1,6.5 \mathrm{~Hz}), 3.56-3.51(2 \mathrm{H}, \mathrm{m}), 3.38$ (1H, ddd, $J=12.3,9.3,4.4 \mathrm{~Hz})$, 3.34-3.25 (3H, m), $3.23(3 \mathrm{H}, \mathrm{s}), 3.25-3.15(5 \mathrm{H}, \mathrm{m}), 3.12-3.06(3 \mathrm{H}, \mathrm{m}), 3.03(1 \mathrm{H}$, ddd, $J=11.0,9.0,4.0 \mathrm{~Hz}), 2.99$ (1H, ddd, $J=11.0,9.0,4.0 \mathrm{~Hz}), 2.94(1 \mathrm{H}, \mathrm{ddd}, J=11.0,9.0$, $4.0 \mathrm{~Hz}), 2.45(1 \mathrm{H}, \mathrm{dd}, J=13.0,3.3 \mathrm{~Hz}), 2.33(1 \mathrm{H}, \mathrm{dt}, J=11.3,4.2 \mathrm{~Hz}), 2.29(1 \mathrm{H}, \mathrm{dd}, J$ 
$=12.1,4.6 \mathrm{~Hz}), 2.23(1 \mathrm{H}, \mathrm{dtd}, J=14.1,7.7,2.2 \mathrm{~Hz}), 2.21-2.17(2 \mathrm{H}, \mathrm{m}), 2.16-2.07(2 \mathrm{H}$, m), $2.03(1 \mathrm{H}, \mathrm{dt}, J=10.6,4.0 \mathrm{~Hz}), 1.96(1 \mathrm{H}, \mathrm{dd}, J=11.9,4.2 \mathrm{~Hz}), 1.87(1 \mathrm{H}, \mathrm{q}, J=11.4$ $\mathrm{Hz}), 1.86(1 \mathrm{H}, \mathrm{m}), 1.79-1.64(5 \mathrm{H}, \mathrm{m}), 1.64-1.55(3 \mathrm{H}, \mathrm{m}), 1.55(1 \mathrm{H}, \mathrm{t}, J=12.3 \mathrm{~Hz})$, $1.50(1 \mathrm{H}, \mathrm{t}, J=11.7 \mathrm{~Hz}), 1.44(1 \mathrm{H}, \mathrm{q}, J=11.6 \mathrm{~Hz}), 1.38(1 \mathrm{H}, \mathrm{q}, J=11.0 \mathrm{~Hz}), 1.31(1 \mathrm{H}$, $\mathrm{t}, J=12.3 \mathrm{~Hz}), 1.28(3 \mathrm{H}, \mathrm{d}, J=5.9 \mathrm{~Hz}), 1.28(3 \mathrm{H}, \mathrm{s}), 1.17(3 \mathrm{H}, \mathrm{s}) ;{ }^{13} \mathrm{C}$ NMR $(150 \mathrm{MHz}$, $\left.\mathrm{CDCl}_{3}\right) \delta 138.6,138.1,128.4,128.3,127.8,127.6(\mathrm{x} 2), 127.4,96.4,83.7,83.1,80.1$, 79.6, 78.7, 78.11, 78.07, 77.83, 77.77, 76.2, 76.1, 75.8, 75.7, 75.3, 74.7, 72.7, 71.5, 70.7, 70.2, 69.3, 68.2, 66.8, 47.1, 44.5, 44.3, 38.6, 37.5, 37.4, 34.7, 32.3, 29.9, 29.6, 29.4, 25.2, 25.0, 17.8, 16.7, 16.2; HRFABMS $m / z$ calcd for $\mathrm{C}_{51} \mathrm{H}_{70} \mathrm{O}_{13} \mathrm{Na}\left(\mathrm{MNa}^{+}\right)$913.4714, found 913.4700. The stereochemistry of the methyl acetal S9 was determined by 2D ROESY experiments and coupling constants in the ${ }^{1} \mathrm{H}$ NMR spectrum.
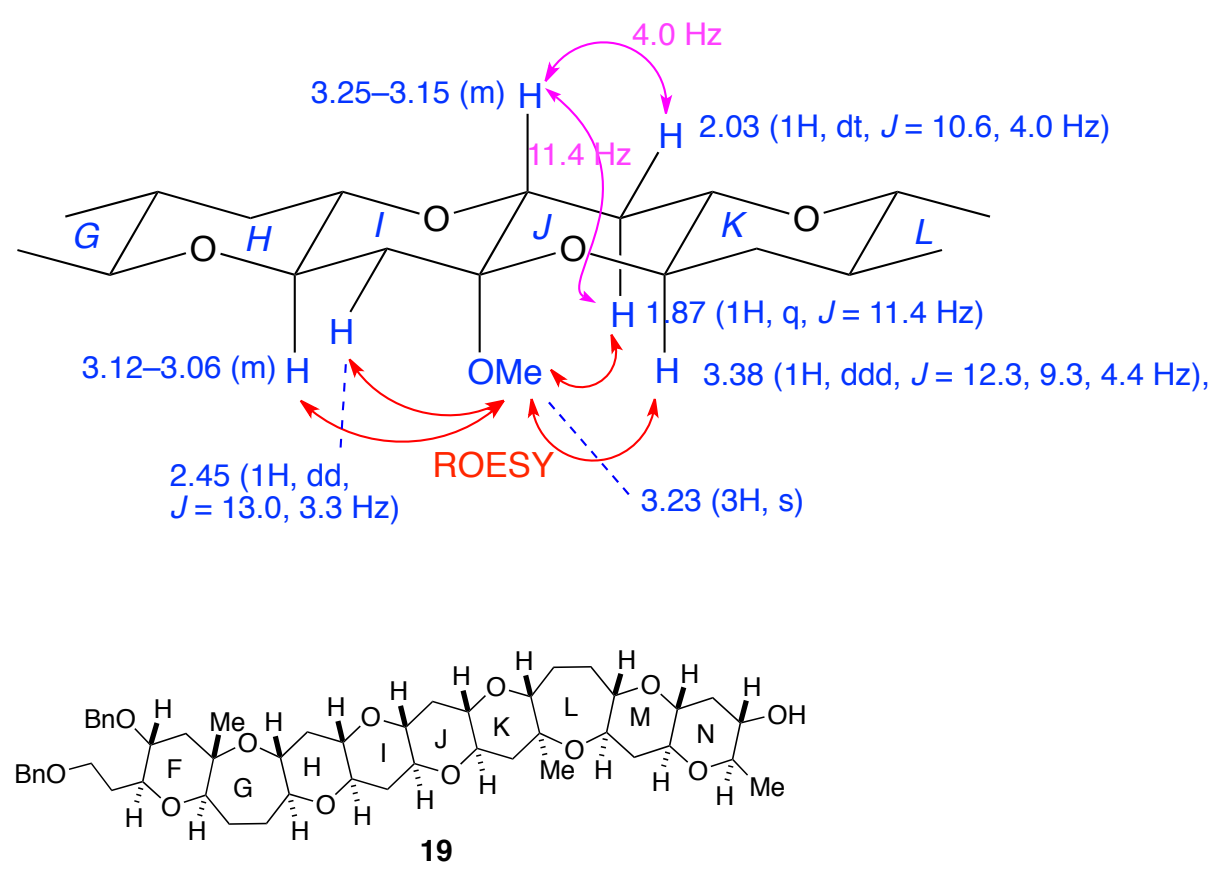

FGHIJKLMN nonacyclic ether 19. To a solution of methyl acetal S9 (64 mg, 0.072 $\mathrm{mmol})$ in $\mathrm{CH}_{2} \mathrm{Cl}_{2}(2.5 \mathrm{~mL})$ at $0{ }^{\circ} \mathrm{C}$ were added $\mathrm{Et}_{3} \mathrm{SiH}(0.195 \mathrm{~mL}, 1.22 \mathrm{mmol})$ and TMSOTf $(0.055 \mathrm{~mL}, 0.31 \mathrm{mmol})$, and the reaction mixture was stirred at $0{ }^{\circ} \mathrm{C}$ for $2 \mathrm{~h}$. The reaction was quenched with saturated aqueous $\mathrm{NaHCO}_{3}$ solution, and the reaction mixture was extracted with EtOAc. The extract was washed with brine, dried, and concentrated under reduced pressure. Purification by flash chromatography $(50 \%$ EtOAc in $\mathrm{CH}_{2} \mathrm{Cl}_{2}$ ) afforded the nonacyclic ether 19 (59 mg, 94\%) as a colorless solid. Mp 231-233 ${ }^{\circ} \mathrm{C} ;[\alpha]^{25}{ }_{\mathrm{D}}+10.2\left(c\right.$ 1.18, $\left.\mathrm{CHCl}_{3}\right)$; IR $\left(\mathrm{CHCl}_{3}\right) 3482,3007,2954,2875$, 1083, $1062 \mathrm{~cm}^{-1} ;{ }^{1} \mathrm{H}$ NMR $\left(600 \mathrm{MHz}, \mathrm{CDCl}_{3}\right) \delta 7.37-7.22(10 \mathrm{H}, \mathrm{m}), 4.60(1 \mathrm{H}, \mathrm{d}, J=$ 
$11.4 \mathrm{~Hz}), 4.52(1 \mathrm{H}, \mathrm{d}, J=12.1 \mathrm{~Hz}), 4.43(1 \mathrm{H}, \mathrm{d}, J=12.1 \mathrm{~Hz}), 4.40(1 \mathrm{H}, \mathrm{d}, J=11.4 \mathrm{~Hz})$, $3.58(2 \mathrm{H}, \mathrm{dd}, J=7.3,6.2 \mathrm{~Hz}), 3.54(1 \mathrm{H}, \mathrm{ddd}, J=11.0,9.4,4.8 \mathrm{~Hz}), 3.52(1 \mathrm{H}, \mathrm{ddd}, J=$ 11.0, 9.4, 4.8 Hz), 3.32-3.20 (5H, m), 3.20-3.14 (2H, m), 3.13-3.04 (4H, m), 3.04-2.95 (4H, m), $2.92(1 \mathrm{H}, \mathrm{ddd}, J=10.6,9.4,4.4 \mathrm{~Hz}), 2.33-2.25(4 \mathrm{H}, \mathrm{m}), 2.22(1 \mathrm{H}, \mathrm{dtd}, J=$ 13.9, 7.7, $2.4 \mathrm{~Hz}), 2.22-2.15(2 \mathrm{H}, \mathrm{m}), 2.15-2.08(2 \mathrm{H}, \mathrm{m}), 2.06(1 \mathrm{H}, \mathrm{dd}, J=11.9,4.2$ $\mathrm{Hz}), 1.97(1 \mathrm{H}, \mathrm{br} \mathrm{s}), 1.86(1 \mathrm{H}, \mathrm{m}), 1.78-1.64(5 \mathrm{H}, \mathrm{m}), 1.61(1 \mathrm{H}, \mathrm{ddt}, J=14.2,8.7,5.8$ $\mathrm{Hz}), 1.53(1 \mathrm{H}, \mathrm{t}, J=12.1 \mathrm{~Hz}), 1.49(1 \mathrm{H}, \mathrm{t}, J=12.1 \mathrm{~Hz}), 1.46(2 \mathrm{H}, \mathrm{q}, J=11.4 \mathrm{~Hz}), 1.43$ $(1 \mathrm{H}, \mathrm{q}, J=11.4 \mathrm{~Hz}), 1.41(1 \mathrm{H}, \mathrm{q}, J=10.4 \mathrm{~Hz}), 1.36(1 \mathrm{H}, \mathrm{q}, J=11.4 \mathrm{~Hz}), 1.26(3 \mathrm{H}, \mathrm{d}, J$ $=5.9 \mathrm{~Hz}), 1.25(3 \mathrm{H}, \mathrm{s}), 1.17(3 \mathrm{H}, \mathrm{s}) ;{ }^{13} \mathrm{C} \mathrm{NMR}\left(150 \mathrm{MHz}, \mathrm{CDCl}_{3}\right) \delta 138.5,138.0,128.3$, $128.2,127.7,127.60,127.58,127.4,83.5,83.0,79.9,79.5,78.00,77.99,77.4,76.9$ (x2), 76.7 (x2), 76.2, 76.0, 75.9, 75.62, 75.57, 75.3, 72.7, 71.3, 70.7, 70.1, 69.2, 66.7, 44.5 (x2), 38.5, 37.5, 37.4, 35.1 (x2), 32.2, 29.5, 29.3, 25.1, 25.0, 17.8, 16.5, 16.1; HRFABMS $m / z$ calcd for $\mathrm{C}_{50} \mathrm{H}_{68} \mathrm{O}_{12} \mathrm{Na}\left(\mathrm{MNa}^{+}\right)$883.4608, found 883.4636.

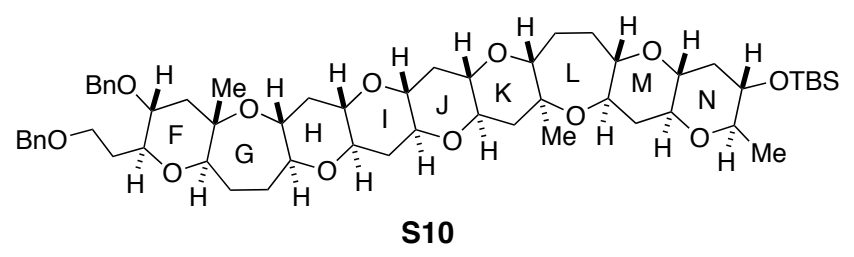

FGHIJKLMN TBS ether S10. To a solution of alcohol 19 (96 mg, $0.111 \mathrm{mmol})$ in $\mathrm{CH}_{2} \mathrm{Cl}_{2}(2 \mathrm{~mL})$ and 2,6 -lutidine $(0.033 \mathrm{~mL}, 0.28 \mathrm{mmol})$ at $0{ }^{\circ} \mathrm{C}$ was added TBSOTf $(0.044 \mathrm{~mL}$. $0.19 \mathrm{mmol})$, and the reaction mixture was stirred at $0{ }^{\circ} \mathrm{C}$ for $15 \mathrm{~min}$. The reaction was quenched with saturated aqueous $\mathrm{NaHCO}_{3}$ solution, and the mixture was extracted with EtOAc. The extract was washed with brine, dried, and concentrated under reduced pressure. Purification by flash chromatography (30\% EtOAc in hexane) afforded TBS ether S10 (110 mg, quant.) as a colorless solid. Mp 197-198 ${ }^{\circ} \mathrm{C} ;[\alpha]^{24}{ }_{\mathrm{D}}$ +20.0 (c 1.18, $\left.\mathrm{CHCl}_{3}\right)$; IR $\left(\mathrm{CHCl}_{3}\right)$ 3006, 2955, 2876, $1068 \mathrm{~cm}^{-1} ;{ }^{1} \mathrm{H} \mathrm{NMR}(600 \mathrm{MHz}$, $\left.\mathrm{CDCl}_{3}\right) \delta 7.35-7.24(10 \mathrm{H}, \mathrm{m}), 4.61(1 \mathrm{H}, \mathrm{d}, J=11.4 \mathrm{~Hz}), 4.52(1 \mathrm{H}, \mathrm{d}, J=12.1 \mathrm{~Hz}), 4.44$ $(1 \mathrm{H}, \mathrm{d}, J=12.1 \mathrm{~Hz}), 4.41(1 \mathrm{H}, \mathrm{d}, J=11.4 \mathrm{~Hz}), 3.58(2 \mathrm{H}, \mathrm{dd}, J=7.5,6.1 \mathrm{~Hz}), 3.55(1 \mathrm{H}$, ddd, $J=11.0,9.3,4.8 \mathrm{~Hz}), 3.53(1 \mathrm{H}$, ddd, $J=11.0,9.3,4.8 \mathrm{~Hz}), 3.32-3.26(3 \mathrm{H}, \mathrm{m})$, 3.26-3.16 (4H, m), 3.10 (1H, ddd, $J=11.5,9.5,4.4 \mathrm{~Hz}), 3.10-3.05$ (3H, m), 3.05-2.95 (4H, m), $2.92(1 \mathrm{H}, \mathrm{ddd}, J=11.5,9.2,4.2 \mathrm{~Hz}), 2.33-2.26(3 \mathrm{H}, \mathrm{m}), 2.26-2.20(2 \mathrm{H}, \mathrm{m})$, $2.18(2 \mathrm{H}, \mathrm{dt}, J=12.1,4.4 \mathrm{~Hz}), 2.16-2.07(2 \mathrm{H}, \mathrm{m}), 2.06(1 \mathrm{H}, \mathrm{dd}, J=12.0,4.3 \mathrm{~Hz}), 1.86$ $(1 \mathrm{H}$, dddd, $J=13.9,7.5,6.0,4.4 \mathrm{~Hz}), 1.79-1.64(5 \mathrm{H}, \mathrm{m}), 1.61$ (1H, ddt, $J=13.9,9.2$, 
$5.9 \mathrm{~Hz}), 1.53(1 \mathrm{H}, \mathrm{t}, J=11.9 \mathrm{~Hz}), 1.49(1 \mathrm{H}, \mathrm{t}, J=11.9 \mathrm{~Hz}), 1.46(2 \mathrm{H}, \mathrm{q}, J=11.9 \mathrm{~Hz})$, $1.44(1 \mathrm{H}, \mathrm{q}, J=11.6 \mathrm{~Hz}), 1.42(1 \mathrm{H}, \mathrm{q}, J=11.4 \mathrm{~Hz}), 1.41(1 \mathrm{H}, \mathrm{q}, J=11.4 \mathrm{~Hz}), 1.26(3 \mathrm{H}$, s), $1.20(3 \mathrm{H}, \mathrm{d}, J=6.1 \mathrm{~Hz}), 1.18(3 \mathrm{H}, \mathrm{s}), 0.87(9 \mathrm{H}, \mathrm{s}), 0.061(3 \mathrm{H}, \mathrm{s}), 0.059(3 \mathrm{H}, \mathrm{s}) ;{ }^{13} \mathrm{C}$ NMR (150 MHz, $\left.\mathrm{CDCl}_{3}\right) \delta 138.6,138.1,128.4,128.3,127.8,127.7,127.6,127.4,83.6$, 83.1, 80.0, 79.5, 78.4, 78.1, 77.5, 77.00, 76.99, 76.8 (x2), 76.2, 76.02, 75.97, 75.7, 75.6, 75.3, 72.7, 72.1, 70.7, 70.1, 69.2, 66.8, 44.6 (x2), 39.2, 37.61, 37.58, 35.2 (x2), 32.3, 29.6, 29.5, 25.7, 25.2, 25.1, 18.2, 17.9, 16.6, 16.2, -4.2, -4.8; HRFABMS $m / z$ calcd for $\mathrm{C}_{56} \mathrm{H}_{82} \mathrm{O}_{12} \mathrm{SiNa}\left(\mathrm{MNa}^{+}\right)$997.5473, found 997.5483. The stereochemisty of the IJ-ring system was determined by chemical shifts and coupling constants.
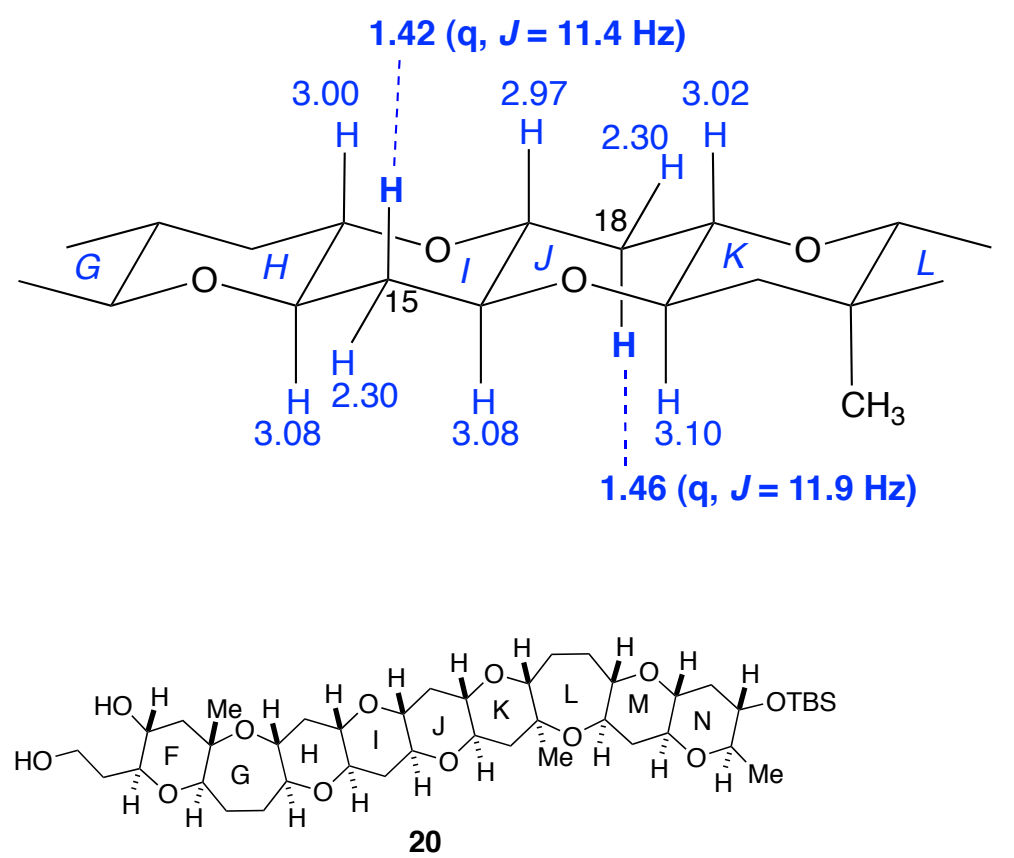

FGHIJKLMN diol 20. A mixture of dibenzyl ether S10 (110 mg, $0.112 \mathrm{mmol}$ ) and $10 \% \mathrm{Pd}(\mathrm{OH})_{2}-\mathrm{C}(14.7 \mathrm{mg})$ in EtOAc/MeOH $(1: 1,4 \mathrm{~mL})$ was stirred under hydrogen atmosphere at $40{ }^{\circ} \mathrm{C}$ for $4 \mathrm{~h}$. The resulting gray suspension was filtered through a Celite pad and the pad was washed with $\mathrm{CH}_{2} \mathrm{Cl}_{2}$. The filtrate and washings were combined and concentrated under reduced pressure. Purification by flash chromatography $(10 \%$ $\mathrm{MeOH}$ in $\mathrm{CH}_{2} \mathrm{Cl}_{2}$ ) afforded diol 20 (85 mg, 95\%) as a colorless solid. Mp 260-262 ${ }^{\circ} \mathrm{C}$; $[\alpha]^{23}{ }_{\mathrm{D}}+48.5\left(c\right.$ 0.29, $\left.\mathrm{CHCl}_{3}\right)$; IR $\left(\mathrm{CHCl}_{3}\right) 3499,3006,2955,2876,1065 \mathrm{~cm}^{-1} ;{ }^{1} \mathrm{H} \mathrm{NMR}$ $\left(600 \mathrm{MHz}\right.$, pyridine- $\left.\mathrm{d}_{5}\right) \delta 6.67(1 \mathrm{H}, \mathrm{d}, J=5.5 \mathrm{~Hz}, \mathrm{OH}), 6.07(1 \mathrm{H}, \mathrm{t}, J=5.0 \mathrm{~Hz}, \mathrm{OH})$, $4.18(2 \mathrm{H}, \mathrm{dt}, J=7.0,5.0 \mathrm{~Hz}), 3.82(1 \mathrm{H}, \mathrm{ddt}, J=11.0,9.7,4.9,4.9 \mathrm{~Hz}), 3.74(1 \mathrm{H}, \mathrm{ddd}, J$ $=11.0,9.2,5.0 \mathrm{~Hz}), 3.72(1 \mathrm{H}, \mathrm{ddd}, J=11.0,9.0,4.9 \mathrm{~Hz}), 3.69(1 \mathrm{H}, \mathrm{td}, J=8.8,3.3 \mathrm{~Hz})$, $3.42(1 \mathrm{H}, \mathrm{ddd}, J=10.6,8.8,4.4 \mathrm{~Hz}), 3.41-3.34(3 \mathrm{H}, \mathrm{m}), 3.33(1 \mathrm{H}, \mathrm{dq}, J=8.8,6.1 \mathrm{~Hz})$, 
3.31-3.25 (3H, m), $3.23(1 \mathrm{H}, \mathrm{ddd}, J=11.4,9.2,4.3 \mathrm{~Hz}), 3.19(1 \mathrm{H}, \mathrm{ddd}, J=11.7,9.5$, $4.0 \mathrm{~Hz}), 3.18-3.14(2 \mathrm{H}, \mathrm{m}), 3.13(1 \mathrm{H}, \mathrm{ddd}, J=11.9,9.2,4.2 \mathrm{~Hz}), 3.06$ (1H, ddd, $J=$ 11.4, 8.9, 4.1 Hz), $2.66(1 \mathrm{H}, \mathrm{dtd}, J=13.8,7.6,3.3 \mathrm{~Hz}), 2.49-2.55(2 \mathrm{H}, \mathrm{m}), 2.47(1 \mathrm{H}, \mathrm{dd}$, $J=12.1,4.8 \mathrm{~Hz}), 2.36-2.44(3 \mathrm{H}, \mathrm{m}), 2.28(1 \mathrm{H}, \mathrm{dd}, J=11.7,4.0 \mathrm{~Hz}), 2.23(1 \mathrm{H}, \mathrm{m}), 2.13$ $(1 \mathrm{H}, \mathrm{dq}, J=14.4,7.5 \mathrm{~Hz}), 2.04(1 \mathrm{H}, \mathrm{ddt}, J=13.8,8.5,5.0 \mathrm{~Hz}), 1.98(1 \mathrm{H}, \mathrm{t}, J=11.9$ $\mathrm{Hz}), 1.94(1 \mathrm{H}, \mathrm{m}), 1.81-1.90(4 \mathrm{H}, \mathrm{m}), 1.82(1 \mathrm{H}, \mathrm{t}, J=12.0 \mathrm{~Hz}), 1.77(1 \mathrm{H}, \mathrm{ddd}, J=14.1$, $12.8,6.8 \mathrm{~Hz}), 1.71(1 \mathrm{H}, \mathrm{q}, J=11.6 \mathrm{~Hz}), 1.70(1 \mathrm{H}, \mathrm{q}, J=11.4 \mathrm{~Hz}), 1.68(2 \mathrm{H}, \mathrm{q}, J=11.2$ $\mathrm{Hz}), 1.60(1 \mathrm{H}, \mathrm{q}, J=11.4 \mathrm{~Hz}), 1.354(3 \mathrm{H}, \mathrm{s}), 1.345(3 \mathrm{H}, \mathrm{s}), 1.28(3 \mathrm{H}, \mathrm{d}, J=6.1 \mathrm{~Hz})$, $0.89(9 \mathrm{H}, \mathrm{s}), 0.083(3 \mathrm{H}, \mathrm{s}), 0.075(3 \mathrm{H}, \mathrm{s}) ;{ }^{13} \mathrm{C}$ NMR $\left(150 \mathrm{MHz}\right.$, pyridine-d $\left.\mathrm{d}_{5}\right) \delta 83.8,83.3$, 81.5, 80.4, 79.9, 78.6, 77.9, 77.4 (x2), 77.2, 77.0, 76.5, 76.4, 76.2, 76.04, 76.01, 72.6, 70.8, 70.0, 69.4, 59.2, 49.6, 45.3, 39.9, 38.4, 38.3, 37.0, 35.95, 35.87, 30.0, 29.8, 25.9, $25.8,25.6,18.6,18.1,16.8,16.4,-4.1,-4.7$; HRFABMS $m / z$ calcd for $\mathrm{C}_{42} \mathrm{H}_{70} \mathrm{O}_{12} \mathrm{SiNa}$ $\left(\mathrm{MNa}^{+}\right)$817.4534, found 817.4543.

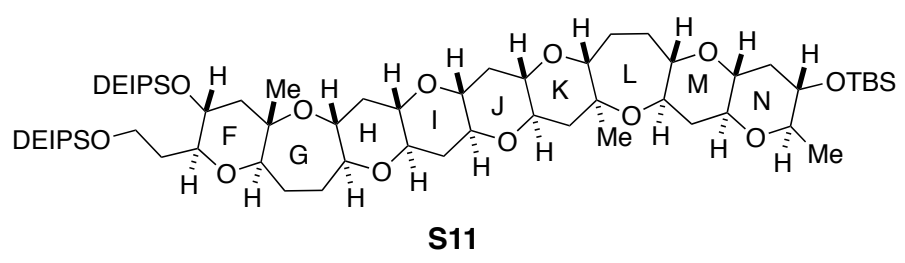

FGHIJKLMN bis(DEIPS) ether S11. To a solution of diol $20(85 \mathrm{mg}, 0.107 \mathrm{mmol})$ in $\mathrm{CH}_{2} \mathrm{Cl}_{2}(2 \mathrm{~mL})$ and 2,6 -lutidine $(0.062 \mathrm{~mL}, 0.535 \mathrm{mmol})$ at $0{ }^{\circ} \mathrm{C}$ was added DEIPSOTf $(0.078 \mathrm{~mL} .0 .321 \mathrm{mmol})$, and the reaction mixture was stirred at $0{ }^{\circ} \mathrm{C}$ for $15 \mathrm{~min}$. The reaction was quenched with saturated aqueous $\mathrm{NaHCO}_{3}$ solution, and the mixture was extracted with EtOAc. The extract was washed with brine, dried, and concentrated under reduced pressure. Purification by flash chromatography (20\% EtOAc in hexane) afforded bis(DEIPS) ether S11 (113 mg, quant.) as a colorless amorphous solid. $[\alpha]^{23}$ +17.6 (c 1.05, $\left.\mathrm{CHCl}_{3}\right)$; IR $\left(\mathrm{CHCl}_{3}\right) 2956,2876,1463,1080,1068 \mathrm{~cm}^{-1} ;{ }^{1} \mathrm{H}$ NMR (600 $\left.\mathrm{MHz}, \mathrm{CDCl}_{3}\right) \delta 3.74(2 \mathrm{H}, \mathrm{dd}, J=8.0,5.0 \mathrm{~Hz}), 3.55(1 \mathrm{H}, \mathrm{dt}, J=9.7,4.9 \mathrm{~Hz}), 3.52(1 \mathrm{H}$, $\mathrm{dt}, J=9.7,4.9 \mathrm{~Hz}), 3.43(1 \mathrm{H}, \mathrm{ddd}, J=11.0,9.1,4.9 \mathrm{~Hz}), 3.29(1 \mathrm{H}$, ddd, $J=10.8,8.8$, $4.6 \mathrm{~Hz}), 3.29-3.16(4 \mathrm{H}, \mathrm{m}), 3.14(1 \mathrm{H}, \mathrm{ddd}, J=10.0,9.0,1.9 \mathrm{~Hz}), 3.10$ (1H, ddd, $J=$ 12.1, 9.4, 4.4 Hz), 3.09-2.94 (7H, m), $2.92(1 \mathrm{H}, \mathrm{ddd}, J=11.4,9.0,4.0 \mathrm{~Hz}), 2.30(1 \mathrm{H}, \mathrm{dt}$, $J=11.4,3.7 \mathrm{~Hz}), 2.29(1 \mathrm{H}, \mathrm{dt}, J=11.4,3.7 \mathrm{~Hz}), 2.22(1 \mathrm{H}, \mathrm{dt}, J=11.7,4.3 \mathrm{~Hz}), 2.18$ $(2 \mathrm{H}, \mathrm{dt}, J=12.1,4.4 \mathrm{~Hz}), 2.05-2.15(3 \mathrm{H}, \mathrm{m}), 2.05(1 \mathrm{H}, \mathrm{dd}, J=11.9,4.2 \mathrm{~Hz}), 2.02(1 \mathrm{H}$, $\mathrm{dd}, J=12.3,4.8 \mathrm{~Hz}), 1.86(1 \mathrm{H}$, dddd, $J=14.4,8.5,6.4,4.4 \mathrm{~Hz}), 1.79-1.64(5 \mathrm{H}, \mathrm{m})$, 
$1.534(1 \mathrm{H}, \mathrm{t}, J=12.0 \mathrm{~Hz}), 1.525(1 \mathrm{H}, \mathrm{t}, J=11.6 \mathrm{~Hz}), 1.46(2 \mathrm{H}, \mathrm{q}, J=11.4 \mathrm{~Hz})$, $1.44-1.35(4 \mathrm{H}, \mathrm{m}), 1.26(3 \mathrm{H}, \mathrm{s}), 1.20(3 \mathrm{H}, \mathrm{d}, J=6.1 \mathrm{~Hz}), 1.20(3 \mathrm{H}, \mathrm{s}), 1.02-0.89(26 \mathrm{H}$, $\mathrm{m}), 0.87(9 \mathrm{H}, \mathrm{s}), 0.65-0.57(8 \mathrm{H}, \mathrm{m}), 0.061(3 \mathrm{H}, \mathrm{s}), 0.059(3 \mathrm{H}, \mathrm{s}) ;{ }^{13} \mathrm{C} \mathrm{NMR}(150 \mathrm{MHz}$, $\left.\mathrm{CDCl}_{3}\right) \delta 83.6,83.0,80.0,79.6,79.5,78.4,77.5,77.01,76.99,76.79,76.76,76.03$, 75.96, 75.7, 75.6, 75.4, 72.1, 70.4, 70.1, 69.2, 59.5, 48.9, 44.6, 39.2, 37.63, 37.60, 35.4, 35.2 (x2), 29.6, 29.5, 25.7, 25.2, 25.1, 18.2, 17.9, 17.3 (x3), 16.6, 16.2, 13.0, 12.8, 7.11, 7.08, 7.0 (x2), 4.1, 3.9, 3.2 (x2), -4.2, -4.8; HRFABMS $m / z$ calcd for $\mathrm{C}_{56} \mathrm{H}_{102} \mathrm{O}_{12} \mathrm{Si}_{3} \mathrm{Na}$ $\left(\mathrm{MNa}^{+}\right)$1073.6577, found 1073.6584.

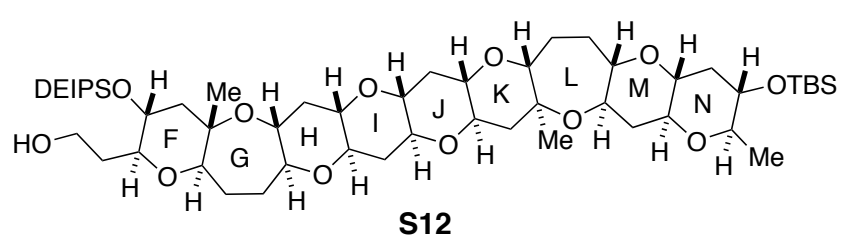

FGHIJKLMN alcohol S12. To a solution of bis(DEIPS) ether S11 (113 mg, 0.107 $\mathrm{mmol})$ in $\mathrm{CH}_{2} \mathrm{Cl}_{2}(1.1 \mathrm{~mL})$ and $\mathrm{MeOH}(1.1 \mathrm{~mL})$ was added PPTS (5.0 mg, $\left.0.021 \mathrm{mmol}\right)$, and the reaction mixture was stirred at $0{ }^{\circ} \mathrm{C}$ for $40 \mathrm{~min}$. The reaction was quenched with $\mathrm{Et}_{3} \mathrm{~N}(0.1 \mathrm{~mL})$, and the reaction mixture was concentrated under reduced pressure. Purification by flash chromatography $(30 \rightarrow 40 \%$ EtOAc in hexane) afforded S11 (89 mg, $90 \%$ ) as a colorless solid. Mp 176-178 ${ }^{\circ} \mathrm{C} ;[\alpha]^{24}{ }_{\mathrm{D}}+27.3\left(\mathrm{c} 1.00, \mathrm{CHCl}_{3}\right)$; IR $\left(\mathrm{CHCl}_{3}\right)$ 3523, 2956, 2877, $1067 \mathrm{~cm}^{-1}$; ${ }^{1} \mathrm{H} \mathrm{NMR}\left(600 \mathrm{MHz}, \mathrm{CDCl}_{3}\right) \delta 3.79$ (2H, q, $J=5.5 \mathrm{~Hz}$ ), 3.57-3.49 (2H, m), 3.50 (1H, ddd, $J=11.0,9.2,4.8 \mathrm{~Hz}), 3.29$ (1H, ddd, $J=10.8,9.0$, $4.6 \mathrm{~Hz}), 3.28-3.21(3 \mathrm{H}, \mathrm{m}), 3.20-3.15(3 \mathrm{H}, \mathrm{m}), 3.13-3.05(3 \mathrm{H}, \mathrm{m}), 3.02(1 \mathrm{H}, \mathrm{ddd}, J=$ 11.3, 9.5, $4.4 \mathrm{~Hz}), 3.01(1 \mathrm{H}, \mathrm{ddd}, J=11.0,9.2,4.4 \mathrm{~Hz}), 2.99-2.94(2 \mathrm{H}, \mathrm{m}), 2.92(1 \mathrm{H}$, ddd, $J=11.5,9.2,4.0 \mathrm{~Hz}), 2.71(1 \mathrm{H}, \mathrm{t}, J=5.5 \mathrm{~Hz}, \mathrm{OH}), 2.30(1 \mathrm{H}, \mathrm{dt}, J=11.8,3.7 \mathrm{~Hz})$, $2.29(1 \mathrm{H}, \mathrm{dt}, J=11.8,3.7 \mathrm{~Hz}), 2.22(1 \mathrm{H}, \mathrm{dt}, J=11.8,4.4 \mathrm{~Hz}), 2.20-2.15(2 \mathrm{H}, \mathrm{m}), 2.12$ $(1 \mathrm{H}, \mathrm{dt}, J=14.5,7.2 \mathrm{~Hz}), 2.11(1 \mathrm{H}, \mathrm{dt}, J=14.5,7.2 \mathrm{~Hz}), 2.08-2.00(3 \mathrm{H}, \mathrm{m}), 1.86(1 \mathrm{H}$, dddd, $J=14.4,8.3,6.4,4.4 \mathrm{~Hz}), 1.82-1.58(6 \mathrm{H}, \mathrm{m}), 1.534(1 \mathrm{H}, \mathrm{t}, J=12.0 \mathrm{~Hz}), 1.530$ $(1 \mathrm{H}, \mathrm{t}, J=12.0 \mathrm{~Hz}), 1.46(2 \mathrm{H}, \mathrm{q}, J=11.4 \mathrm{~Hz}), 1.42(1 \mathrm{H}, \mathrm{q}, J=11.4 \mathrm{~Hz}), 1.41(2 \mathrm{H}, \mathrm{q}, J$ $=11.4 \mathrm{~Hz}), 1.26(3 \mathrm{H}, \mathrm{s}), 1.22(3 \mathrm{H}, \mathrm{s}), 1.20(3 \mathrm{H}, \mathrm{d}, J=6.1 \mathrm{~Hz}), 1.00-0.95(12 \mathrm{H}, \mathrm{m})$, 0.89 (1H, septet, $J=6.8 \mathrm{~Hz}), 0.87(9 \mathrm{H}, \mathrm{s}), 0.67-0.58(4 \mathrm{H}, \mathrm{m}), 0.060(3 \mathrm{H}, \mathrm{s}), 0.059(3 \mathrm{H}$, s); ${ }^{13} \mathrm{C}$ NMR (150 MHz, $\left.\mathrm{CDCl}_{3}\right) \delta 84.0,83.6,83.1,79.8,79.6,78.4,77.5,77.0$ (x2), 76.8, 76.7, 76.02, 75.97, 75.7, 75.6, 75.1, 72.1, 70.1, 70.0, 69.6, 61.9, 48.5, 44.6, 39.2, $37.6,37.5,35.19,35.16,33.9,29.5,29.4,25.7,25.4,25.1,18.2,17.9,17.3(x 2), 16.6$, 
16.1, 12.9, 7.1, 7.0, 4.0, 3.8, -4.2, -4.8; HRFABMS m/z calcd for $\mathrm{C}_{49} \mathrm{H}_{86} \mathrm{O}_{12} \mathrm{Si}_{2} \mathrm{Na}$ $\left(\mathrm{MNa}^{+}\right)$945.5556, found 945.5566.

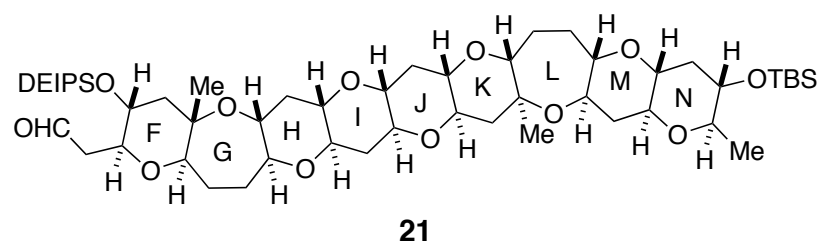

FGHIJKLMN aldehyde 21. To a solution of alcohol S12 (95 $\mathrm{mg}, 0.103 \mathrm{mmol})$ in $\mathrm{CH}_{2} \mathrm{Cl}_{2}(3.0 \mathrm{~mL})$ was added Dess-Martin periodinane $(88 \mathrm{mg}, 0.206 \mathrm{mmol})$, and the reaction mixture was stirred at room temperature for $2 \mathrm{~h}$. The reaction was quenched with a 1:1 mixture of saturated aqueous $\mathrm{Na}_{2} \mathrm{~S}_{2} \mathrm{O}_{3}$ solution and saturated aqueous $\mathrm{NaHCO}_{3}$ solution, and the mixture was extracted with EtOAc. The extract was washed with and brine, dried, and concentrated under reduced pressure. Purification by flash chromatography (30\% EtOAc in hexane) afforded aldehyde 21 (89 $\mathrm{mg}, 93 \%)$ as a colorless solid. Mp 180-182 ${ }^{\circ} \mathrm{C}$; $[\alpha]^{21}{ }_{\mathrm{D}}+28.1$ (c 0.95, $\left.\mathrm{CHCl}_{3}\right)$; IR $\left(\mathrm{CHCl}_{3}\right) 2956,2877$, 1727, 1080, $1069 \mathrm{~cm}^{-1} ;{ }^{1} \mathrm{H}$ NMR $\left(600 \mathrm{MHz}, \mathrm{CDCl}_{3}\right) \delta 9.75(1 \mathrm{H}, \mathrm{dd}, J=2.9,1.7 \mathrm{~Hz})$, $3.58(1 \mathrm{H}, \mathrm{td}, J=8.8,3.3 \mathrm{~Hz}), 3.48-3.56(3 \mathrm{H}, \mathrm{m}), 3.29(1 \mathrm{H}, \mathrm{ddd}, J=10.8,9.0,4.8 \mathrm{~Hz})$, 3.27-3.16 (5H, m), 3.13-3.05 (3H, m), 3.05-2.94 (4H, m), 2.92 (1H, ddd, $J=11.2,8.8$, $4.0 \mathrm{~Hz}), 2.76(1 \mathrm{H}, \mathrm{ddd}, J=16.1,3.3,1.7 \mathrm{~Hz}), 2.43(1 \mathrm{H}, \mathrm{ddd}, J=16.1,8.8,3.1 \mathrm{~Hz}), 2.30$ $(1 \mathrm{H}, \mathrm{dt}, J=11.8,3.7 \mathrm{~Hz}), 2.29(1 \mathrm{H}, \mathrm{dt}, J=11.8,4.7 \mathrm{~Hz}), 2.22(1 \mathrm{H}, \mathrm{dt}, J=11.7,4.3 \mathrm{~Hz})$, 2.20-2.15 (2H, m), 2.15-2.08 (2H, m), 2.07 (1H, dd, $J=11.8,5.0 \mathrm{~Hz}), 2.05(1 \mathrm{H}, \mathrm{dd}, J$ $=11.8,5.0 \mathrm{~Hz}), 1.86(1 \mathrm{H}, \mathrm{dddd}, J=14.4,8.3,6.4,4.4 \mathrm{~Hz}), 1.80-1.64(5 \mathrm{H}, \mathrm{m}), 1.57(1 \mathrm{H}$, t, $J=11.8 \mathrm{~Hz}), 1.53(1 \mathrm{H}, \mathrm{t}, J=11.8 \mathrm{~Hz}), 1.46(2 \mathrm{H}, \mathrm{q}, J=11.4 \mathrm{~Hz}), 1.42(1 \mathrm{H}, \mathrm{q}, J=$ $11.4 \mathrm{~Hz}), 1.41(2 \mathrm{H}, \mathrm{q}, J=11.4 \mathrm{~Hz}), 1.26(3 \mathrm{H}, \mathrm{s}), 1.21(3 \mathrm{H}, \mathrm{s}), 1.20(3 \mathrm{H}, \mathrm{d}, J=6.1 \mathrm{~Hz})$, $0.98(3 \mathrm{H}, \mathrm{d}, J=6.9 \mathrm{~Hz}), 0.972(3 \mathrm{H}, \mathrm{d}, J=6.9 \mathrm{~Hz}), 0.971(3 \mathrm{H}, \mathrm{t}, J=7.9 \mathrm{~Hz}), 0.96(3 \mathrm{H}, \mathrm{t}$, $J=7.9 \mathrm{~Hz}), 0.89(1 \mathrm{H}, \mathrm{spt}, J=6.9 \mathrm{~Hz}), 0.87(9 \mathrm{H}, \mathrm{s}), 0.67-0.56(4 \mathrm{H}, \mathrm{m}), 0.060(3 \mathrm{H}, \mathrm{s})$, $0.059(3 \mathrm{H}, \mathrm{s}) ;{ }^{13} \mathrm{C} \mathrm{NMR}\left(150 \mathrm{MHz}, \mathrm{CDCl}_{3}\right) \delta$ 201.4, 83.6, 83.3, 79.9, 79.5, 78.4, 78.3, 77.5, 77.0 (x2), 76.8, 76.7, 76.02, 75.96, 75.7, 75.6, 75.2, 72.1, 70.1, 69.9, 69.6, 48.5, 46.2 , 44.6, 39.2, 37.6, 37.5, 35.17, 35.16, 29.5, 29.4, 25.7, 25.09, 25.08, 18.2, 17.9, $17.25,17.22,16.6,16.2,12.9,7.1,7.0,4.0,3.8,-4.2,-4.8$; HRESIMS $m / z$ calcd for $\mathrm{C}_{50} \mathrm{H}_{88} \mathrm{O}_{12} \mathrm{Si}_{2} \mathrm{Na}\left(\mathrm{M}+\mathrm{CH}_{3} \mathrm{OH}+\mathrm{Na}^{+}\right)$975.5661, found 975.5658 . 


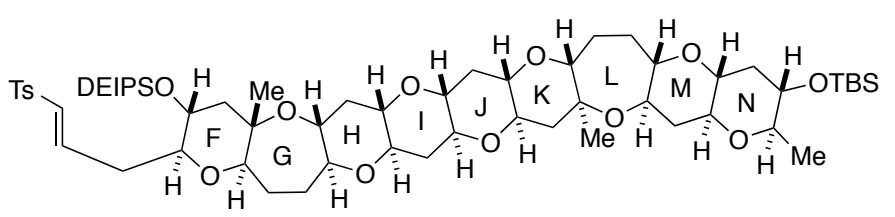

22

FGHIJKLMN vinyl sulfone 22. To a solution of aldehyde 21 (89 mg, $0.096 \mathrm{mmol}$ ), diethyl $p$-toluenesulfonymethylphosphonate $(36.0 \mathrm{mg}, 0.12 \mathrm{mmol})$, and $\mathrm{LiCl}(5.0 \mathrm{mg}$, $0.12 \mathrm{mmol})$ in THF $(2.0 \mathrm{~mL})$ was added $\mathrm{Et}_{3} \mathrm{~N}(0.017 \mathrm{~mL}, 0.12 \mathrm{mmol})$, and the reaction mixture was stirred at room temperature for $44 \mathrm{~h}$. The same amounts of diethyl p-toluenesulfonymethylphosphonate, $\mathrm{LiCl}$, and $\mathrm{Et}_{3} \mathrm{~N}(0.12 \mathrm{mmol}$ each) were added, and the reaction mixture was stirred for additional $5 \mathrm{~h}$. The reaction was quenched with saturated aqueous $\mathrm{NH}_{4} \mathrm{Cl}$ solution. The reaction mixture was extracted with EtOAc, and the extract was washed with brine, dried, and concentrated under reduced pressure. Purification by flash chromatography (30\% EtOAc in hexane) afforded vinyl sulfone 22 $(E: Z=95: 5,98 \mathrm{mg}, 95 \%)$ as a colorless solid. Mp $138-141{ }^{\circ} \mathrm{C} ;[\alpha]^{24} \mathrm{D}+39.2(c) 1.46$, $\left.\mathrm{CHCl}_{3}\right)$; IR $\left(\mathrm{CHCl}_{3}\right)$ 2956, 2877, 1727, 1082, $1068 \mathrm{~cm}^{-1} ;{ }^{1} \mathrm{H}$ NMR for the major $E$-isomer (600 MHz, $\left.\mathrm{CDCl}_{3}\right) \delta 7.75(2 \mathrm{H}, \mathrm{d}, J=8.2 \mathrm{~Hz}), 7.31(2 \mathrm{H}, \mathrm{d}, J=8.2 \mathrm{~Hz}), 6.99$ $(1 \mathrm{H}, \mathrm{dt}, J=15.1,7.2 \mathrm{~Hz}), 6.35(1 \mathrm{H}, \mathrm{d}, J=15.1 \mathrm{~Hz}), 3.53(2 \mathrm{H}, \mathrm{td}, J=9.3,5.0 \mathrm{~Hz}), 3.40$ (1H, ddd, $J=10.9,9.2,4.9 \mathrm{~Hz}), 3.29$ (1H, ddd, $J=10.6,8.8,4.6 \mathrm{~Hz}), 3.27-3.15(4 \mathrm{H}$, m), 3.15-3.05 (5H, m), 3.05-2.95 (4H, m), 2.92 (1H, ddd, $J=11.5,9.0,4.2 \mathrm{~Hz}), 2.69$ (1H, dddd, $J=14.8,7.2,2.6,1.1 \mathrm{~Hz}), 2.43(3 \mathrm{H}, \mathrm{s}), 2.34-2.27(2 \mathrm{H}, \mathrm{m}), 2.27-2.15(4 \mathrm{H}$, m), 2.14-2.08 (2H, m), $2.06(1 \mathrm{H}, \mathrm{dd}, J=12.1,4.2 \mathrm{~Hz}), 2.01(1 \mathrm{H}, \mathrm{dd}, J=12.3,4.8 \mathrm{~Hz})$, $1.86(1 \mathrm{H}$, dddd, $J=14.4,8.3,6.4,4.4 \mathrm{~Hz}), 1.78-1.63(5 \mathrm{H}, \mathrm{m}), 1.54(1 \mathrm{H}, \mathrm{t}, J=12.0 \mathrm{~Hz})$, $1.51(1 \mathrm{H}, \mathrm{t}, J=11.6 \mathrm{~Hz}), 1.45(1 \mathrm{H}, \mathrm{q}, J=11.4 \mathrm{~Hz}), 1.44(1 \mathrm{H}, \mathrm{q}, J=11.4 \mathrm{~Hz}), 1.42(1 \mathrm{H}$, q, $J=11.4 \mathrm{~Hz}), 1.41(2 \mathrm{H}, \mathrm{q}, J=11.4 \mathrm{~Hz}), 1.26(3 \mathrm{H}, \mathrm{s}), 1.20(3 \mathrm{H}, \mathrm{d}, J=6.2 \mathrm{~Hz}), 1.17$ $(3 \mathrm{H}, \mathrm{s}), 0.96-0.91(12 \mathrm{H}, \mathrm{m}), 0.87(9 \mathrm{H}, \mathrm{s}), 0.86(1 \mathrm{H}, \mathrm{m}), 0.62-0.51(4 \mathrm{H}, \mathrm{m}), 0.061(3 \mathrm{H}$, s), $0.060(3 \mathrm{H}, \mathrm{s}) ;{ }^{13} \mathrm{C} \mathrm{NMR}$ for the major $E$-isomer $\left(150 \mathrm{MHz}, \mathrm{CDCl}_{3}\right) \delta 144.1,143.4$, $137.8,132.3,129.8,127.6,83.6,83.1,81.0,79.9,79.5,78.4,77.4,77.0$ (x2), 76.74, 76.71, 76.0, 75.9, 75.7, 75.6, 75.1, 72.1, 70.1, 69.7, 69.5, 48.5, 44.6, 39.2, 37.6, 37.5, 35.2, 35.1, 33.8, 29.4 (x2), 25.7, 25.1 (x2), 21.6, 18.2, 17.9, 17.24, 17.21, 16.6, 16.1, 12.9, 7.1, 7.0, 4.0, 3.8, -4.2, -4.8; HRFABMS $m / z$ calcd for $\mathrm{C}_{57} \mathrm{H}_{92} \mathrm{O}_{13} \mathrm{SSi}_{2} \mathrm{Na}\left(\mathrm{MNa}^{+}\right)$ 1095.5695, found 1095.5724. 


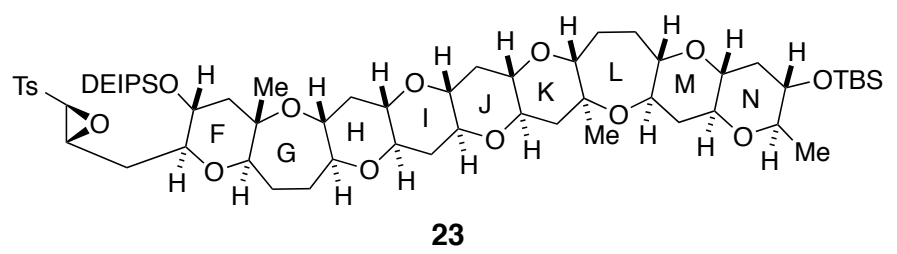

FGHIJKLMN epoxy sulfone 23. To a solution of $t$-BuOK (24 mg, $0.212 \mathrm{mmol})$ in THF $(1 \mathrm{~mL})$ at $-80{ }^{\circ} \mathrm{C}$ were added $t$-BuOOH $(0.058 \mathrm{~mL}$ of a $5.5 \mathrm{M}$ solution in nonane, $0.32 \mathrm{mmol}$ ) and a solution of vinyl sulfone 22 (98 $\mathrm{mg}, 0.092 \mathrm{mmol})$ in THF (2 mL). The reaction mixture was stirred at $-80{ }^{\circ} \mathrm{C}$ for $10 \mathrm{~min}$ and warmed to $-20{ }^{\circ} \mathrm{C}$ over $1 \mathrm{~h}$, and then the mixture was stirred at $-20{ }^{\circ} \mathrm{C}$ for $3 \mathrm{~h}$, before the reaction was quenched with saturated aqueous $\mathrm{NH}_{4} \mathrm{Cl}$ solution. The reaction mixture was extracted with EtOAc, and the extract was washed with brine, dried, and concentrated under reduced pressure. Purification by flash chromatography $\left(10 \rightarrow 15 \%\right.$ EtOAc in $\left.\mathrm{CH}_{2} \mathrm{Cl}_{2}\right)$ afforded trans-epoxy sulfone 23 (66:34 diastereomeric mixture, $92 \mathrm{mg}, 92 \%$ ) as a colorless oil. Cis-epoxy sulfones were removed by this chromatography. Mp $146-148{ }^{\circ} \mathrm{C} ;[\alpha]^{21} \mathrm{D}$ +27.9 (c 0.96, $\left.\mathrm{CHCl}_{3}\right)$; IR $\left(\mathrm{CHCl}_{3}\right) 2956,2877,1081,1066 \mathrm{~cm}^{-1} ;{ }^{1} \mathrm{H}$ NMR for the major isomer (600 MHz, $\left.\mathrm{CDCl}_{3}\right) \delta 7.80(2 \mathrm{H}, \mathrm{d}, J=8.3 \mathrm{~Hz}), 7.37(2 \mathrm{H}, \mathrm{d}, J=8.3 \mathrm{~Hz}), 3.91(1 \mathrm{H}$, $\mathrm{d}, J=1.7 \mathrm{~Hz}), 3.75(1 \mathrm{H}, \mathrm{td}, J=5.5,1.7 \mathrm{~Hz}), 3.55-3.51(2 \mathrm{H}, \mathrm{m}), 3.46(1 \mathrm{H}, \mathrm{ddd}, J=11.0$, 9.0, $4.8 \mathrm{~Hz}), 3.30(1 \mathrm{H}$, ddd, $J=10.5,8.6,4.6 \mathrm{~Hz}), 3.27-3.22(2 \mathrm{H}, \mathrm{m}), 3.21-3.17(2 \mathrm{H}$, m), 3.17-3.06 (5H, m), 3.05-2.95 (4H, m), 2.92 (1H, ddd, $J=11.4,9.0,4.2 \mathrm{~Hz}), 2.46$ $(3 \mathrm{H}, \mathrm{s}), 2.32-2.28(2 \mathrm{H}, \mathrm{m}), 2.22(1 \mathrm{H}, \mathrm{dt}, J=11.7,4.4 \mathrm{~Hz}), 2.20-2.16(2 \mathrm{H}, \mathrm{m})$, 2.15-2.08 (2H, m), 2.08-2.00 (3H, m), $1.86(1 \mathrm{H}, \mathrm{dddd}, J=14.4,8.3,6.4,4.4 \mathrm{~Hz})$, 1.81-1.65 (6H, m), 1.50-1.32 (7H, m), $1.26(3 \mathrm{H}, \mathrm{s}), 1.21(3 \mathrm{H}, \mathrm{d}, J=6.2 \mathrm{~Hz}), 1.20(3 \mathrm{H}$, s), 0.97-0.92 (12H, m), $0.87(9 \mathrm{H}, \mathrm{s}), 0.87(1 \mathrm{H}, \mathrm{m}), 0.62-0.55(4 \mathrm{H}, \mathrm{m}), 0.06(6 \mathrm{H}, \mathrm{s}) ;{ }^{1} \mathrm{H}$ NMR for the minor isomer $\left(600 \mathrm{MHz}, \mathrm{CDCl}_{3}\right) \delta 7.80(2 \mathrm{H}, \mathrm{d}, J=8.3 \mathrm{~Hz}), 7.38(2 \mathrm{H}, \mathrm{d}, J$ $=8.3 \mathrm{~Hz}), 3.91(1 \mathrm{H}, \mathrm{d}, J=1.7 \mathrm{~Hz}), 3.79(1 \mathrm{H}, \mathrm{ddd}, J=6.8,5.3,1.7 \mathrm{~Hz}), 3.55-3.51(2 \mathrm{H}$, m), $3.40(1 \mathrm{H}$, ddd, $J=10.8,9.0,4.8 \mathrm{~Hz}), 3.30$ (1H, ddd, $J=10.5,8.6,4.6 \mathrm{~Hz})$, 3.27-3.22 (2H, m), 3.21-3.17 (3H, m), 3.17-3.06 (4H, m), 3.05-2.95 (4H, m), $2.92(1 \mathrm{H}$, ddd, $J=11.4,9.0,4.2 \mathrm{~Hz}), 2.46(3 \mathrm{H}, \mathrm{s}), 2.32-2.28(2 \mathrm{H}, \mathrm{m}), 2.22(1 \mathrm{H}, \mathrm{dt}, J=11.7,4.4$ $\mathrm{Hz}), 2.20-2.16(2 \mathrm{H}, \mathrm{m}), 2.15-2.08(2 \mathrm{H}, \mathrm{m}), 2.08-2.00(3 \mathrm{H}, \mathrm{m}), 1.86(1 \mathrm{H}, \mathrm{dddd}, J=$ 14.4, 8.3, 6.4, 4.4 Hz), 1.81-1.65 (5H, m), $1.56(1 \mathrm{H}, \mathrm{m}), 1.50-1.32(7 \mathrm{H}, \mathrm{m}), 1.26(3 \mathrm{H}$, s), $1.21(3 \mathrm{H}, \mathrm{d}, J=6.2 \mathrm{~Hz}), 1.20(3 \mathrm{H}, \mathrm{s}), 0.97-0.92(12 \mathrm{H}, \mathrm{m}), 0.87(9 \mathrm{H}, \mathrm{s}), 0.87(1 \mathrm{H}$, $\mathrm{m}), 0.62-0.55(4 \mathrm{H}, \mathrm{m}), 0.06(6 \mathrm{H}, \mathrm{s}) ;{ }^{13} \mathrm{C} \mathrm{NMR}$ for the major isomer $\left(150 \mathrm{MHz}, \mathrm{CDCl}_{3}\right)$ ठ 145.5, 134.1, 129.9, 128.7, 83.6, 83.0, 79.9 (x2), 79.5, 78.4, 77.5, 77.0 (x2), 76.8, 76.7, 
76.0, 75.9, 75.7, 75.6, 75.1, 72.1, 70.1, 69.6 (x2), 68.0, 55.8, 48.5, 44.6, 39.2, 37.6, 37.5, $35.18,35.16,32.5,29.5,29.4,25.7,25.15,25.08,21.7,18.2$, 17.9, 17.3, 17.2, 16.6, 16.2, 12.9, 7.1, 7.0, 4.0, 3.8, $-4.2,-4.8 ;{ }^{13} \mathrm{C} \mathrm{NMR}$ for the minor isomer $\left(150 \mathrm{MHz}, \mathrm{CDCl}_{3}\right) \delta$ 145.5, 134.2, 130.0, 128.7, 83.6, 83.0, 79.9, 79.5, 79.4, 78.4, 77.5, 77.0 (x2), 76.8, 76.7, 76.0, 75.9, 75.7, 75.6, 75.2, 72.1, 70.2, 70.1, 69.6, 69.0, 55.6, 48.6, 44.6, 39.2, 37.6, $37.5,35.18,35.16,33.5,29.5,29.4,25.7,25.1,24.9,21.7,18.2$, 17.9, 17.24, 17.22, 16.6, 16.2, 12.9, 7.1, 7.0, 4.0, 3.8, -4.2, -4.8; HRFABMS $m / z$ calcd for $\mathrm{C}_{57} \mathrm{H}_{92} \mathrm{O}_{14} \mathrm{SSi}_{2} \mathrm{Na}$ $\left(\mathrm{MNa}^{+}\right)$1111.5644, found 1111.5725 .
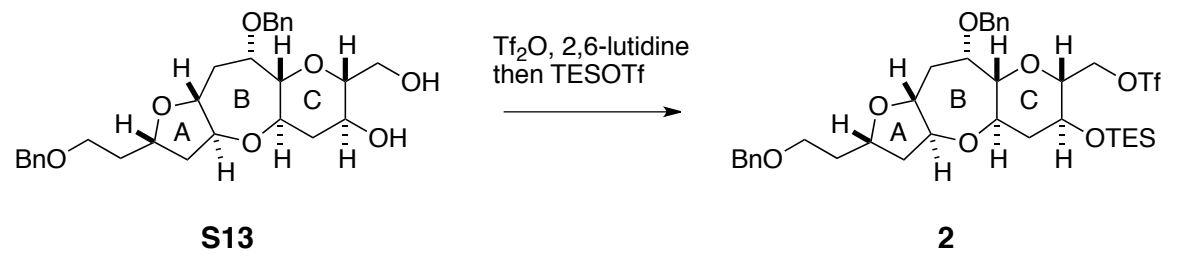

ABC triflate 2. To a solution of ABC diol S13 (126 mg, 0.260 mmol) in 2,6-lutidine (0.091 mL, $0.78 \mathrm{mmol})$ and $\mathrm{CH}_{2} \mathrm{Cl}_{2}(1 \mathrm{~mL})$ at $-80{ }^{\circ} \mathrm{C}$ was added $\mathrm{Tf}_{2} \mathrm{O}(0.045 \mathrm{~mL}, 0.27$ $\mathrm{mmol})$, and the reaction mixture was stirred at $-80^{\circ} \mathrm{C}$ for $30 \mathrm{~min}$. TESOTf $(0.065 \mathrm{~mL}$, $0.29 \mathrm{mmol}$ ) was then added, and the reaction mixture was stirred at $-80{ }^{\circ} \mathrm{C}$ for $30 \mathrm{~min}$. The reaction was quenched with saturated aqueous $\mathrm{NaHCO}_{3}$ solution, and the reaction mixture was extracted with EtOAc. The extract was washed with brine, dried, and concentrated under reduced pressure. Purification by flash chromatography $(15 \rightarrow 25 \%$ EtOAc in hexane) afforded triflate $2(162 \mathrm{mg}, 85 \%)$ as a colorless oil. $[\alpha]^{23}{ }_{\mathrm{D}}+23.8(c$ 1.28, $\left.\mathrm{CHCl}_{3}\right)$; IR $\left(\mathrm{CHCl}_{3}\right)$ 2957, 2877, 1415, 1146, 1094, $956 \mathrm{~cm}^{-1} ;{ }^{1} \mathrm{H}$ NMR (600 MHz, $\left.\mathrm{CDCl}_{3}\right) \delta$ 7.36-7.25 $(10 \mathrm{H}, \mathrm{m}), 4.71(1 \mathrm{H}, \mathrm{dd}, J=10.5,1.7 \mathrm{~Hz}), 4.65$ and 4.61 (each $1 \mathrm{H}$, $\mathrm{d}, J=12.1 \mathrm{~Hz}), 4.55(1 \mathrm{H}, \mathrm{dd}, J=10.5,5.6 \mathrm{~Hz}), 4.50$ and 4.47 (each $1 \mathrm{H}, \mathrm{d}, J=11.9 \mathrm{~Hz}$ ), $4.27(1 \mathrm{H}, \mathrm{ddd}, J=9.0,7.8,6.8 \mathrm{~Hz}), 4.14(1 \mathrm{H}$, quintet, $J=6.9 \mathrm{~Hz}), 4.01(1 \mathrm{H}, \mathrm{d}, J=6.4$ $\mathrm{Hz}), 3.93(1 \mathrm{H}, \mathrm{ddd}, J=11.5,9.1,4.6 \mathrm{~Hz}), 3.61-3.56(2 \mathrm{H}, \mathrm{m}), 3.55(2 \mathrm{H}, \mathrm{t}, J=6.4 \mathrm{~Hz})$, $3.31(1 \mathrm{H}, \mathrm{ddd}, J=9.0,5.6,1.7 \mathrm{~Hz}), 3.28(1 \mathrm{H}, \mathrm{d}, J=9.2 \mathrm{~Hz}), 2.44-2.37(2 \mathrm{H}, \mathrm{m})$, 2.00-1.84 (4H, m), $1.80(1 \mathrm{H}, \mathrm{dq}, J=12.8,6.8 \mathrm{~Hz}), 1.54(1 \mathrm{H}, \mathrm{q}, J=11.6 \mathrm{~Hz}), 0.95(9 \mathrm{H}$, $\mathrm{t}, J=8.0 \mathrm{~Hz}), 0.611(3 \mathrm{H}, \mathrm{dq}, J=15.4,8.0 \mathrm{~Hz}), 0.607(3 \mathrm{H}, \mathrm{dq}, J=15.4,8.0 \mathrm{~Hz}) ;{ }^{13} \mathrm{C}$ NMR $\left(150 \mathrm{MHz}, \mathrm{CDCl}_{3}\right) \delta 138.9,138.4,128.3,128.2,127.6,127.5,127.4,127.3$, $118.6\left(\mathrm{q}, J_{C-F}=319.5 \mathrm{~Hz}\right), 83.1,82.0,79.0,78.9,77.9,75.3,75.1,73.0,72.9,70.0,67.2$, 65.6, 40.0, 38.1, 36.3, 33.3, 6.7, 4.9; HRFABMS $m / z$ calcd for $\mathrm{C}_{35} \mathrm{H}_{49} \mathrm{O}_{9} \mathrm{~F}_{3} \mathrm{SSiNa}$ $\left(\mathrm{MNa}^{+}\right)$753.2716, found 753.2688. 
ABC-FGHIJKLMN Coupling product 24. To a solution of ABC-triflate 2 (123 mg, $0.168 \mathrm{mmol}$ ) and FGHIJKLMN-epoxy sulfone 23 (66:34 diastereomeric mixture, $92 \mathrm{mg}$, $0.084 \mathrm{mmol})$ in THF $(2 \mathrm{~mL})$ and HMPA $(0.111 \mathrm{~mL}, 0.636 \mathrm{mmol})$ at $-100{ }^{\circ} \mathrm{C}$ was added $n$-BuLi $(0.079 \mathrm{~mL}$ of a $1.60 \mathrm{M}$ solution in hexane, $0.126 \mathrm{mmol})$, and the reaction mixture was stirred at $-100{ }^{\circ} \mathrm{C}$ for $0.5 \mathrm{~h}$. The reaction was quenched with saturated aqueous $\mathrm{NH}_{4} \mathrm{Cl}$ solution. The reaction mixture was extracted with EtOAc, and the extract was washed with brine, dried, and concentrated under reduced pressure. Purification by flash chromatography $\left(30 \%\right.$ EtOAc in $\left.\mathrm{CH}_{2} \mathrm{Cl}_{2}\right)$ afforded coupling product 24 (66:34 diastereomeric mixture, $131 \mathrm{mg}$, 92\%). The diastereomers were partially separated by repeated chromatography.

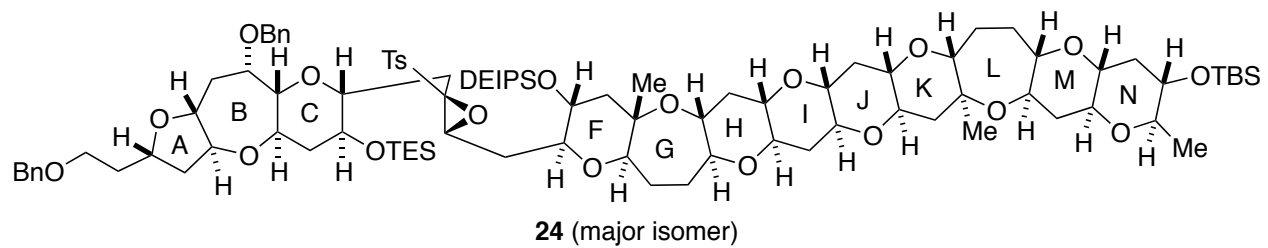

Major coupling product. Colorless amorphous solid; $[\alpha]^{23}{ }_{\mathrm{D}}+37.9\left(c 1.37, \mathrm{CHCl}_{3}\right)$; IR $\left(\mathrm{CHCl}_{3}\right)$ 2956, 2877, 1456, 1083, $1068 \mathrm{~cm}^{-1} ;{ }^{1} \mathrm{H} \mathrm{NMR}\left(600 \mathrm{MHz}, \mathrm{CDCl}_{3}\right) \delta 7.82(2 \mathrm{H}, \mathrm{d}$, $J=8.3 \mathrm{~Hz}$ ), 7.35-7.29 (8H, m), 7.29-7.24 (4H, m), 4.58 and 4.57 (each 1H, d, $J=12.5$ $\mathrm{Hz}), 4.48$ and 4.46 (each 1H, d, $J=11.9 \mathrm{~Hz}), 4.15(1 \mathrm{H}, \mathrm{ddd}, J=9.0,7.7,6.6 \mathrm{~Hz}), 4.11$ $(1 \mathrm{H}, \mathrm{dq}, J=12.3,6.9 \mathrm{~Hz}), 3.80(1 \mathrm{H}, \mathrm{t}, J=6.0 \mathrm{~Hz}), 3.78(1 \mathrm{H}, \mathrm{ddd}, J=11.6,9.2,4.8 \mathrm{~Hz})$, $3.73(1 \mathrm{H}, \mathrm{d}, J=6.4 \mathrm{~Hz}), 3.50-3.56(4 \mathrm{H}, \mathrm{m}), 3.48(1 \mathrm{H}, \mathrm{ddd}, J=10.9,9.4,4.9 \mathrm{~Hz}), 3.43$ $(1 \mathrm{H}, \mathrm{q}, J=8.1 \mathrm{~Hz}), 3.29(1 \mathrm{H}, \mathrm{ddd}, J=10.8,8.8,4.6 \mathrm{~Hz}), 3.27-3.06(10 \mathrm{H}, \mathrm{m})$, 3.05-2.95 (5H, m), $2.92(1 \mathrm{H}, \mathrm{ddd}, J=11.7,9.0,4.2 \mathrm{~Hz}), 2.84(1 \mathrm{H}, \mathrm{dd}, J=15.6,1.3 \mathrm{~Hz})$, $2.66(1 \mathrm{H}, \mathrm{d}, J=9.0 \mathrm{~Hz}), 2.43(3 \mathrm{H}, \mathrm{s}), 2.31(1 \mathrm{H}, \mathrm{dt}, J=11.4,3.9 \mathrm{~Hz}), 2.30(1 \mathrm{H}, \mathrm{dt}, J=$ 11.4, 3.9 Hz), 2.27-2.20 (3H, m), 2.18 (2H, dt, $J=12.1,4.3 \mathrm{~Hz}), 2.14-2.09$ (2H, m), 2.08-2.00 (3H, m), 1.96-1.81 (5H, m), 1.80-1.63 (8H, m), $1.54(1 \mathrm{H}, \mathrm{t}, J=12.1 \mathrm{~Hz})$, $1.50(1 \mathrm{H}, \mathrm{t}, J=12.1 \mathrm{~Hz}), 1.464(1 \mathrm{H}, \mathrm{q}, J=11.4 \mathrm{~Hz}), 1.458(1 \mathrm{H}, \mathrm{q}, J=11.4 \mathrm{~Hz}), 1.44$ $(1 \mathrm{H}, \mathrm{q}, J=11.4 \mathrm{~Hz}), 1.42(1 \mathrm{H}, \mathrm{q}, J=11.4 \mathrm{~Hz}), 1.41(1 \mathrm{H}, \mathrm{q}, J=11.4 \mathrm{~Hz}), 1.31(1 \mathrm{H}, \mathrm{q}, J$ $=11.6 \mathrm{~Hz}), 1.26(3 \mathrm{H}, \mathrm{s}), 1.21(3 \mathrm{H}, \mathrm{d}, J=6.1 \mathrm{~Hz}), 1.17(3 \mathrm{H}, \mathrm{s}), 0.97-0.90(21 \mathrm{H}, \mathrm{m})$, $0.87(9 \mathrm{H}, \mathrm{s}), 0.87(1 \mathrm{H}, \mathrm{m}), 0.63-0.53(10 \mathrm{H}, \mathrm{m}), 0.061(3 \mathrm{H}, \mathrm{s}), 0.059(3 \mathrm{H}, \mathrm{s}) ;{ }^{13} \mathrm{C} \mathrm{NMR}$ $\left(150 \mathrm{MHz}, \mathrm{CDCl}_{3}\right) \delta 144.7,139.2,138.4,134.7,129.5,129.4,128.3,128.2,127.6$, $127.5,127.4,127.2,83.6,83.1,82.5,82.1,80.8,79.9,79.6,78.9,78.4,78.1,77.8,77.5$, 77.00, 76.99, 76.8, 76.7, 76.0, 75.9, 75.7, 75.6, 75.2, 75.0, 74.2, 73.0, 72.8, 72.1, 70.4, 
$70.3,70.1,69.7,69.5,67.2,57.7,48.6,44.6,40.2,39.2,38.1,37.6,37.5,36.2,35.19$, $35.16,33.4,30.1,29.5,29.4,28.9,25.7,25.2,25.1,21.7,18.2,17.9,17.30,17.27,16.6$, 16.1, 12.9, 7.13, 7.07, 6.9, 5.0, 4.1, 3.8, -4.2, -4.8; HRESIMS $\mathrm{m} / \mathrm{z}$ calcd for $\mathrm{C}_{91} \mathrm{H}_{140} \mathrm{O}_{20} \mathrm{SSi}_{3} \mathrm{Na}\left(\mathrm{MNa}^{+}\right)$1691.8864, found 1691.8878 .

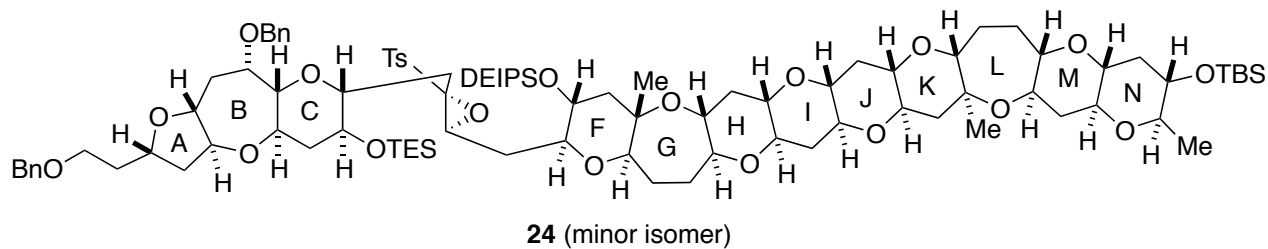

Minor coupling product. Colorless amorphous solid; $[\alpha]_{\mathrm{D}}^{22}+0.0\left(c 1.24, \mathrm{CHCl}_{3}\right)$; IR $\left(\mathrm{CHCl}_{3}\right)$ 2956, 2876, 1456, 1084, $1066 \mathrm{~cm}^{-1} ;{ }^{1} \mathrm{H}$ NMR $\left(600 \mathrm{MHz}, \mathrm{CDCl}_{3}\right) \delta 7.80(2 \mathrm{H}, \mathrm{d}$, $J=8.3 \mathrm{~Hz}$ ), 7.36-7.29 (10H, m), 7.28-7.24 (2H, m), 4.60 and 4.52 (each 1H, d, $J=$ $11.9 \mathrm{~Hz}$ ), 4.46 and 4.45 (each 1H, d, $J=11.9 \mathrm{~Hz}), 4.30$ (1H, q, $J=7.8 \mathrm{~Hz}$ ), 4.16-4.09 $(2 \mathrm{H}, \mathrm{m}), 3.97(1 \mathrm{H}, \mathrm{d}, J=6.1 \mathrm{~Hz}), 3.94(1 \mathrm{H}, \mathrm{ddd}, J=11.4,9.3,4.8 \mathrm{~Hz}), 3.67$ (1H, ddd, $J$ $=11.5,8.9,2.6 \mathrm{~Hz}), 3.60(1 \mathrm{H}, \mathrm{q}, J=8.3 \mathrm{~Hz}), 3.56-3.48(4 \mathrm{H}, \mathrm{m}), 3.29(1 \mathrm{H}, \mathrm{ddd}, J=$ 10.6, 8.8, 4.6 Hz), 3.26-3.22 (3H, m), 3.21-3.16 (4H, m), 3.15-3.06 (5H, m), 3.05-2.95 (4H, m), 2.92 (1H, ddd, $J=11.4,9.0,4.2 \mathrm{~Hz}), 2.43$ (3H, s), 2.42-2.27 (5H, m), 2.23 $(1 \mathrm{H}, \mathrm{dt}, J=11.6,4.3 \mathrm{~Hz}), 2.20-2.08(4 \mathrm{H}, \mathrm{m}), 2.06(1 \mathrm{H}, \mathrm{dd}, J=12.2,4.3 \mathrm{~Hz}), 1.93(2 \mathrm{H}$, t, $J=7.7 \mathrm{~Hz}), 1.91(1 \mathrm{H}, \mathrm{dd}, J=12.2,4.3 \mathrm{~Hz}), 1.88-1.80(5 \mathrm{H}, \mathrm{m}), 1.79-1.62(6 \mathrm{H}, \mathrm{m})$, $1.541(1 \mathrm{H}, \mathrm{q}, J=11.6 \mathrm{~Hz}), 1.539(1 \mathrm{H}, \mathrm{t}, J=12.0 \mathrm{~Hz}), 1.46(1 \mathrm{H}, \mathrm{q}, J=11.4 \mathrm{~Hz})$, 1.46-1.38 (6H, m), $1.26(3 \mathrm{H}, \mathrm{s}), 1.21(3 \mathrm{H}, \mathrm{d}, J=6.1 \mathrm{~Hz}), 1.05(3 \mathrm{H}, \mathrm{s}), 0.87(9 \mathrm{H}, \mathrm{s})$, $0.849(9 \mathrm{H}, \mathrm{t}, J=8.0 \mathrm{~Hz}), 0.848(3 \mathrm{H}, \mathrm{d}, J=7.3 \mathrm{~Hz}), 0.84(3 \mathrm{H}, \mathrm{d}, J=7.3 \mathrm{~Hz}), 0.82(3 \mathrm{H}, \mathrm{t}$, $J=8.0 \mathrm{~Hz}), 0.81(3 \mathrm{H}, \mathrm{t}, J=8.0 \mathrm{~Hz}), 0.71(1 \mathrm{H}$, septet, $J=7.3 \mathrm{~Hz}), 0.55-0.45(6 \mathrm{H}, \mathrm{m})$, 0.45-0.31 (4H, m), $0.061(3 \mathrm{H}, \mathrm{s}), 0.060(3 \mathrm{H}, \mathrm{s}) ;{ }^{13} \mathrm{C} \mathrm{NMR}\left(150 \mathrm{MHz}, \mathrm{CDCl}_{3}\right) \delta$ 145.1, 139.0, 138.4, 133.1, 129.8, 129.4, 128.3, 128.2, 127.54, 127.46, 127.2, 126.5, 83.6, 83.0, 82.7, 82.2, 80.2, 79.9, 79.5, 78.7, 78.4 (x2), 78.0, 77.5, 77.0 (x2), 76.7 (x2), 76.0, 75.9, 75.7, 75.6, 75.1, 74.9, 74.0, 73.0, 72.1, 71.5, 70.8, 70.5, 70.1, 70.0, 69.5, 67.2, 59.0, 48.6, 44.6, 40.6, 39.2, 38.1, 37.6, 37.5, 36.2, 35.2, 35.1, 32.1, 31.9, 30.5, 29.4 (x2), 25.7, 25.1 (x2), 21.6, 18.2, 17.9, 17.2 (x2), 16.6, 16.1, 12.8, 7.1, 7.0, 6.7, 4.9, 3.9, 3.7, -4.2, -4.8; HRESIMS $m / z$ calcd for $\mathrm{C}_{91} \mathrm{H}_{140} \mathrm{O}_{20} \mathrm{SSi}_{3} \mathrm{Na}\left(\mathrm{MNa}^{+}\right)$1691.8864, found 1691.8872. 


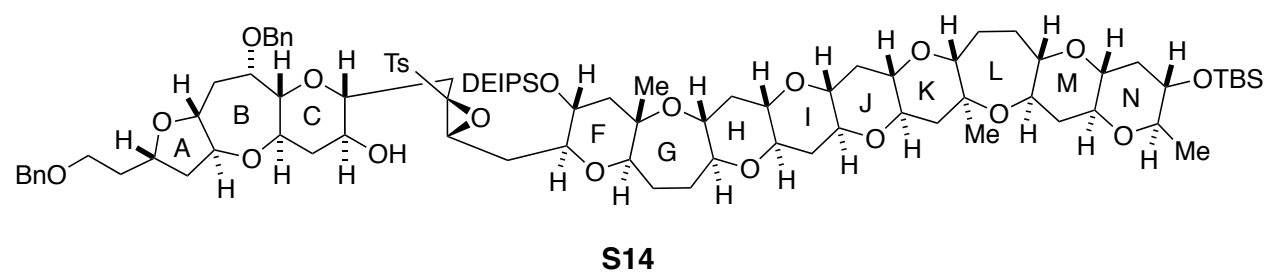

ABC-FGHIJKLMN epoxy alcohol S14. To a solution of the coupling product 24 (66:34 diastereomeric mixture, $124 \mathrm{mg}, 0.0744 \mathrm{mmol})$ in $\mathrm{CH}_{2} \mathrm{Cl}_{2}(0.75 \mathrm{~mL}$ ) and $\mathrm{EtOH}$ $(0.75 \mathrm{~mL})$ was added $( \pm)$-10-camphorsulfonic acid $(3.9 \mathrm{mg}, 0.017 \mathrm{mmol})$, and the reaction mixture was stirred at $0{ }^{\circ} \mathrm{C}$ for $3.5 \mathrm{~h}$. The reaction was quenched with $\mathrm{Et}_{3} \mathrm{~N}(0.1$ $\mathrm{mL}$ ), and the reaction mixture was concentrated under reduced pressure. Alcohol S14 $(68 \mathrm{mg})$ and the remaining starting TES ether $24(44 \mathrm{mg})$ were separated by flash chromatography $(40 \rightarrow 50 \%$ EtOAc in hexane). The recovered TES ether was subjected to the same protocol twice to afford additional alcohol S14 $(31 \mathrm{mg})$. In total, $99 \mathrm{mg}$ (65:35 diastereomeric mixture, 85\%) of $\mathbf{S 1 4}$ was obtained as a colorless solid. Mp $122-126{ }^{\circ} \mathrm{C} ;[\alpha]^{21}+20.7$ (c 0.91, $\left.\mathrm{CHCl}_{3}\right)$; IR $\left(\mathrm{CHCl}_{3}\right) 3517,2950,2874,1455,1082$ $\mathrm{cm}^{-1} ;{ }^{1} \mathrm{H} \mathrm{NMR}$ for the major isomer $\left(600 \mathrm{MHz}, \mathrm{CDCl}_{3}\right) \delta 7.81(2 \mathrm{H}, \mathrm{d}, J=8.3 \mathrm{~Hz})$, 7.35-7.24 (12H, m), $4.58(2 \mathrm{H}, \mathrm{s}), 4.48$ and 4.47 (each $1 \mathrm{H}, \mathrm{d}, J=11.9 \mathrm{~Hz}), 4.19(1 \mathrm{H}$, ddd, $J=9.0,8.0,7.0 \mathrm{~Hz}), 4.14-4.10(1 \mathrm{H}, \mathrm{m}), 3.90-3.85(1 \mathrm{H}, \mathrm{m}), 3.83(1 \mathrm{H}, \mathrm{d}, J=6.4$ $\mathrm{Hz}), 3.68(1 \mathrm{H}, \mathrm{t}, J=5.9 \mathrm{~Hz}), 3.59-3.57(5 \mathrm{H}, \mathrm{m}), 3.46(1 \mathrm{H}, \mathrm{ddd}, J=11.0,9.0,4.8 \mathrm{~Hz})$, 3.32-2.89 (18H, m), $2.57(1 \mathrm{H}, \mathrm{dd}, J=15.8,4.0 \mathrm{~Hz}), 2.44(3 \mathrm{H}, \mathrm{s}), 2.44-2.38(1 \mathrm{H}, \mathrm{m})$, 2.37-2.27 (3H, m), $2.23(1 \mathrm{H}, \mathrm{dt}, J=11.7,4.2 \mathrm{~Hz}), 2.20-2.04(6 \mathrm{H}, \mathrm{m}), 2.02(1 \mathrm{H}, \mathrm{dd}, J=$ 12.5, $4.8 \mathrm{~Hz}), 1.97-1.90(4 \mathrm{H}, \mathrm{m}), 1.88-1.64(10 \mathrm{H}, \mathrm{m}), 1.56-1.32(8 \mathrm{H}, \mathrm{m}), 1.26(3 \mathrm{H}, \mathrm{s})$, $1.21(3 \mathrm{H}, \mathrm{d}, J=6.1 \mathrm{~Hz}), 1.16(3 \mathrm{H}, \mathrm{s}), 0.94-0.76(13 \mathrm{H}, \mathrm{m}), 0.87(9 \mathrm{H}, \mathrm{s}), 0.60-0.43(4 \mathrm{H}$, $\mathrm{m}), 0.06(6 \mathrm{H}, \mathrm{s}) ;{ }^{1} \mathrm{H} \mathrm{NMR}$ for the minor isomer $\left(600 \mathrm{MHz}, \mathrm{CDCl}_{3}\right) \delta 7.79(2 \mathrm{H}, \mathrm{d}, J=$ $8.4 \mathrm{~Hz}$ ), 7.35-7.24 (12H, m), 4.54 and 4.53 (each $1 \mathrm{H}, \mathrm{d}, J=12.5 \mathrm{~Hz}$ ), 4.47 and 4.45 (each $1 \mathrm{H}, \mathrm{d}, J=11.9 \mathrm{~Hz}), 4.25(1 \mathrm{H}, \mathrm{q}, J=8.0 \mathrm{~Hz}), 4.14-4.10(1 \mathrm{H}, \mathrm{m}), 3.96-3.91(2 \mathrm{H}$, m), 3.90-3.85 (1H, m), 3.59-3.57 (5H, m), $3.38(1 \mathrm{H}, \mathrm{td}, J=8.5,3.7 \mathrm{~Hz}), 3.32-2.89$ (18H, m), 2.49 (1H, dd, $J=16.0,3.5 \mathrm{~Hz}), 2.43$ (3H, s), 2.44-2.38 (1H, m), 2.37-2.27 $(3 \mathrm{H}, \mathrm{m}), 2.23(1 \mathrm{H}, \mathrm{dt}, J=11.7,4.2 \mathrm{~Hz}), 2.20-2.04(6 \mathrm{H}, \mathrm{m}), 1.97-1.90(5 \mathrm{H}, \mathrm{m})$, 1.88-1.64 (10H, m), 1.56-1.32 (8H, m), $1.26(3 \mathrm{H}, \mathrm{s}), 1.21(3 \mathrm{H}, \mathrm{d}, J=6.1 \mathrm{~Hz}), 1.10(3 \mathrm{H}$, s), $0.94-0.76(13 \mathrm{H}, \mathrm{m}), 0.87(9 \mathrm{H}, \mathrm{s}), 0.60-0.43(4 \mathrm{H}, \mathrm{m}), 0.06(6 \mathrm{H}, \mathrm{s}) ;{ }^{13} \mathrm{C}$ NMR for the major isomer $\left(150 \mathrm{MHz}, \mathrm{CDCl}_{3}\right) \delta 145.1,138.9,138.4,133.2,129.6,129.5,128.3$, 128.1, 127.5 (x2), 127.4, 127.3, 83.6, 83.0, 82.7, 82.0, 80.3, 79.8, 79.5, 78.8, 78.3, 77.82, 77.77, 77.4, 76.9 (x2), 76.71, 76.67, 76.0, 75.9, 75.7, 75.6, 75.1, 75.0, 74.1, 72.9, 
$72.5,72.1,70.4,70.1,69.65,69.61,69.5,67.1,58.4,48.5,44.5,40.2,39.1,38.0,37.6$, $37.5,36.2,35.13,35.11,33.3,29.9,29.40$ (x2), 29.36, 25.7, 25.1, 25.0, 21.7, 18.1, 17.8, 17.24, 17.21, 16.5, 16.0, 12.8, 7.1, 7.0, 4.0, 3.8, $-4.2,-4.8 ;{ }^{13} \mathrm{C}$ NMR for the minor isomer $\left(150 \mathrm{MHz}, \mathrm{CDCl}_{3}\right) \delta 145.2,138.8,138.4,132.8,129.7,129.6,128.3,128.2$, $127.5,127.3,127.2,126.8,83.6,82.9,82.7,82.0,80.0,79.8,79.5,78.5,78.4,78.3,77.9$, 77.4, 76.9 (x2), 76.71, 76.67, 76.0, 75.9, 75.7, 75.6, 75.1, 74.9, 74.1, 72.9, 72.1, 72.0, $70.4,70.10,70.06,69.61,69.56,67.1,59.4,48.5,44.5,40.0,39.1,38.0,37.6,37.5,36.2$, $35.13,35.11,32.5,29.8,29.4$ (x2), 29.3, 25.7, 25.0, 24.9, 21.6, 18.1, 17.8, 17.21, 17.18, 16.5, 16.1, 12.8, 7.1, 7.0, 3.9, 3.7, -4.2, -4.8; HRFABMS $m / z$ calcd for $\mathrm{C}_{85} \mathrm{H}_{126} \mathrm{O}_{20} \mathrm{SSi}_{2} \mathrm{Na}\left(\mathrm{MNa}^{+}\right)$1577.7999, found 1577.8007 .

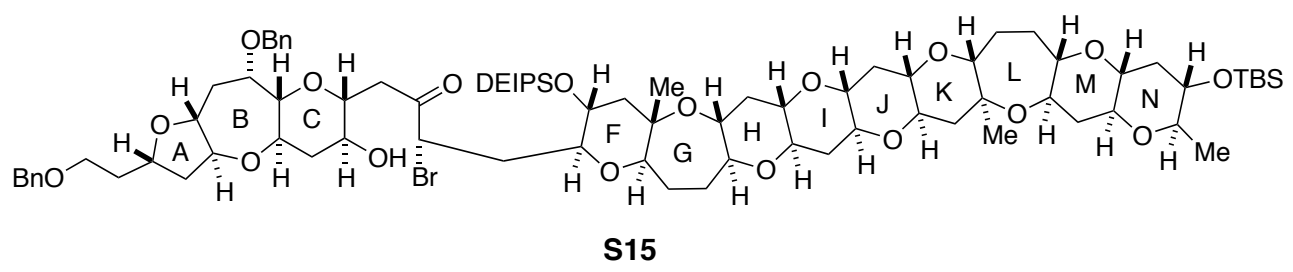

ABC-FGHIJKLMN bromoketone S15. To a solution of S14 (65:35 diastereomeric mixture, $99 \mathrm{mg}, 0.064 \mathrm{mmol})$ and $\mathrm{LiBr}(17 \mathrm{mg}, 0.19 \mathrm{mmol})$ in $\mathrm{CH}_{2} \mathrm{Cl}_{2}(1 \mathrm{~mL})$ at $-15^{\circ} \mathrm{C}$ was added $\mathrm{MgBr}_{2} \cdot \mathrm{OEt}_{2}$ (50 mg, $0.19 \mathrm{mmol}$ ), and the mixture was stirred at $0{ }^{\circ} \mathrm{C}$ for 17 h. The reaction was quenched with saturated aqueous $\mathrm{NaHCO}_{3}$ solution, and the reaction mixture was extracted with EtOAc, and the extract was washed with water, and brine, dried, and concentrated under reduced pressure. Purification by flash chromatography $(40 \rightarrow 50 \%$ EtOAc in hexane) afforded bromoketone S15 (71:29 diastereomeric mixture, $91 \mathrm{mg}, 97 \%)$ as a colorless amorphous solid. $[\alpha]^{25}+2.3(c$ 1.69, $\left.\mathrm{CHCl}_{3}\right)$; IR $\left(\mathrm{CHCl}_{3}\right) 3459,2951,2874,1718,1086 \mathrm{~cm}^{-1} ;{ }^{1} \mathrm{H}$ NMR for the major isomer (600 MHz, $\left.\mathrm{CDCl}_{3}\right)$ d 7.34-7.28 (8H, m), 7.28-7.24 (2H, m), 4.58 and 4.57 (each $1 \mathrm{H}, \mathrm{d}, J=12.1 \mathrm{~Hz}), 4.59-4.53(1 \mathrm{H}, \mathrm{m}), 4.48$ and 4.46 (each $1 \mathrm{H}, \mathrm{d}, J=11.9 \mathrm{~Hz}$ ), 4.26-4.20 (1H, m), 4.15-4.10 (1H, m), 3.96-3.90 (2H, m), 3.60-3.37 (8H, m), $3.29(1 \mathrm{H}$, ddd, $J=10.6,8.8,4.6 \mathrm{~Hz}), 3.25-3.17(5 \mathrm{H}, \mathrm{m}), 3.13-2.87(12 \mathrm{H}, \mathrm{m}), 2.44-2.34(3 \mathrm{H}, \mathrm{m})$, 2.32-2.26 (2H, m), $2.22(1 \mathrm{H}, \mathrm{dt}, J=11.6,4.2 \mathrm{~Hz}), 2.20-2.15(2 \mathrm{H}, \mathrm{m}), 2.14-2.04(4 \mathrm{H}$, m), 2.02-1.98 (1H, m), 1.96-1.90 (2H, m), 1.88-1.61 (9H, m), 1.55-1.38 (8H, m), 1.31-1.24 (1H, m), $1.26(3 \mathrm{H}, \mathrm{s}), 1.20(3 \mathrm{H}, \mathrm{d}, J=6.1 \mathrm{H}), 1.10(3 \mathrm{H}, \mathrm{s}), 1.00-0.95(12 \mathrm{H}$, $\mathrm{m}), 0.90-0.85(1 \mathrm{H}, \mathrm{m}), 0.87(9 \mathrm{H}, \mathrm{s}), 0.66-0.58(4 \mathrm{H}, \mathrm{m}), 0.060(3 \mathrm{H}, \mathrm{s}), 0.058(3 \mathrm{H}, \mathrm{s}) ;{ }^{1} \mathrm{H}$ NMR for the minor isomer $\left(600 \mathrm{MHz}, \mathrm{CDCl}_{3}\right) \delta 7.34-7.28(8 \mathrm{H}, \mathrm{m}), 7.28-7.24(2 \mathrm{H}, \mathrm{m})$, 
4.60 and 4.59 (each $1 \mathrm{H}, \mathrm{d}, J=12.1 \mathrm{~Hz}$ ), 4.59-4.53 (1H, m), 4.48 and 4.46 (each $1 \mathrm{H}, \mathrm{d}$, $J=11.9 \mathrm{~Hz}), 4.26-4.20(1 \mathrm{H}, \mathrm{m}), 4.15-4.10(1 \mathrm{H}, \mathrm{m}), 3.96-3.90(2 \mathrm{H}, \mathrm{m}), 3.60-3.37(8 \mathrm{H}$, m), $3.29(1 \mathrm{H}, \mathrm{ddd}, J=10.6,8.8,4.6 \mathrm{~Hz}), 3.25-3.17(5 \mathrm{H}, \mathrm{m}), 3.13-2.87(12 \mathrm{H}, \mathrm{m}), 2.66$ $(1 \mathrm{H}, \mathrm{ddd}, J=13.6,10.3,2.6 \mathrm{~Hz}), 2.44-2.34(2 \mathrm{H}, \mathrm{m}), 2.32-2.26(2 \mathrm{H}, \mathrm{m}), 2.22(1 \mathrm{H}, \mathrm{dt}, J$ $=11.6,4.2 \mathrm{~Hz}), 2.20-2.15(2 \mathrm{H}, \mathrm{m}), 2.14-2.04(4 \mathrm{H}, \mathrm{m}), 2.02-1.98(1 \mathrm{H}, \mathrm{m}), 1.96-1.90$ $(2 \mathrm{H}, \mathrm{m}), 1.88-1.61(9 \mathrm{H}, \mathrm{m}), 1.55-1.38(8 \mathrm{H}, \mathrm{m}), 1.31-1.24(1 \mathrm{H}, \mathrm{m}), 1.26(3 \mathrm{H}, \mathrm{s}), 1.20$ $(3 \mathrm{H}, \mathrm{d}, J=6.1 \mathrm{H}), 1.15(3 \mathrm{H}, \mathrm{s}), 1.00-0.95(12 \mathrm{H}, \mathrm{m}), 0.90-0.85(1 \mathrm{H}, \mathrm{m}), 0.87(9 \mathrm{H}, \mathrm{s})$, $0.66-0.58(4 \mathrm{H}, \mathrm{m}), 0.060(3 \mathrm{H}, \mathrm{s}), 0.058(3 \mathrm{H}, \mathrm{s}) ;{ }^{13} \mathrm{C}$ NMR for the major isomer $(150$ $\left.\mathrm{MHz}, \mathrm{CDCl}_{3}\right) \delta 201.7,138.9,138.4,128.3,128.2,127.5,127.43,127.36,127.3,83.6$, 83.0, 82.9, 82.0, 79.8, 79.7, 79.5, 78.9, 78.4, 78.0, 77.7, 77.4, 76.9 (x2), 76.71, 76.68, $76.0,75.9,75.7,75.6,75.1,75.0,72.9,72.7,72.1,70.5,70.10,70.06,69.6,68.8,67.2$, 50.7, 48.6, 44.5, 42.4, 39.9, 39.1, 38.0, 37.6, 37.4, 36.2, 35.5, 35.1 (x2), 33.3, 29.39, 29.36, 25.7, 25.1, 25.0, 18.1, 17.8, 17.2 (x2), 16.5, 16.0, 12.9, 7.04, 7.01, 4.0, 3.8, -4.2, $-4.8 ;{ }^{13} \mathrm{C}$ NMR for the minor isomer $\left(150 \mathrm{MHz}, \mathrm{CDCl}_{3}\right) \delta 202.0,138.9,138.4,128.3$, $128.2,127.5,127.4,127.29,127.27,83.6,82.90,82.89,82.0,80.0,79.7,79.5,78.8$, 78.4, 78.3, 77.7, 77.4, 76.9 (x2), 76.71, 76.65, 76.0, 75.9, 75.7, 75.6, 75.02, 74.96, 72.9, $72.7,72.1,70.5,70.1,69.7,69.6,69.0,67.2,50.3,48.5,44.5,41.6,40.0,39.1,38.0$, $37.6,37.4,36.9,36.2,35.1$ (x2), 33.2, 29.4, 29.3, 25.7, 25.0, 24.9, 18.1, 17.8, 17.2 (x2), $16.5,16.0,12.9,7.04,7.01,4.0,3.8,-4.2,-4.8$; HRFABMS $\mathrm{m} / z$ calcd for $\mathrm{C}_{78} \mathrm{H}_{119} \mathrm{O}_{18} \mathrm{BrSi}_{2} \mathrm{Na}\left(\mathrm{MNa}^{+}\right)$1501.7016, found 1501.6992 .

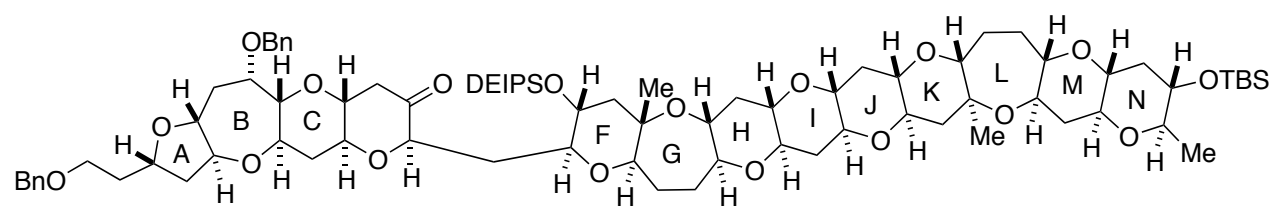

25

ABCD'-FGHIJKLMN ketone 25. To a solution of bromoketone S15 (91 mg, 0.062 $\mathrm{mmol})$ in $\mathrm{CH}_{2} \mathrm{Cl}_{2}(3.4 \mathrm{~mL})$ was added $\mathrm{DBU}(0.0102 \mathrm{~mL}, 0.068 \mathrm{mmol})$ at $0{ }^{\circ} \mathrm{C}$, and the reaction mixture was stirred at $0{ }^{\circ} \mathrm{C}$ for $3 \mathrm{~h}$. The reaction was quenched with saturated aqueous $\mathrm{NH}_{4} \mathrm{Cl}$ solution, and the mixture was extracted with EtOAc. The extract was washed with brine, dried, and concentrated. Purification by flash chromatography $(30 \rightarrow 50 \%$ EtOAc in hexane) afforded cyclic ketone 25 (single diastereomer, $76 \mathrm{mg}$, $88 \%$ ) as a colorless solid. Mp $152-153{ }^{\circ} \mathrm{C} ;[\alpha]^{23} \mathrm{D}+15.3\left(c 1.13, \mathrm{CHCl}_{3}\right)$; IR $\left(\mathrm{CHCl}_{3}\right)$ 2950, 2860, 1727, 1455, $1082 \mathrm{~cm}^{-1} ;{ }^{1} \mathrm{H}$ NMR (600 MHz, $\left.\mathrm{CDCl}_{3}\right) \delta 7.36-7.30(8 \mathrm{H}, \mathrm{m})$, 
7.30-7.25 (2H, m), 4.65 and 4.59 (each $1 \mathrm{H}, \mathrm{d}, J=11.9 \mathrm{~Hz}$ ), 4.50 and 4.47 (each $1 \mathrm{H}, \mathrm{d}$, $J=11.9 \mathrm{~Hz}), 4.28(1 \mathrm{H}, \mathrm{ddd}, J=9.0,8.0,7.0 \mathrm{~Hz}), 4.15(1 \mathrm{H}$, quintet, $J=7.0 \mathrm{~Hz}), 4.06$ $(1 \mathrm{H}, \mathrm{ddd}, J=11.4,9.0,4.9 \mathrm{~Hz}), 4.01(1 \mathrm{H}, \mathrm{dd}, J=6.1,4.1 \mathrm{~Hz}), 3.98(1 \mathrm{H}, \mathrm{d}, J=6.4 \mathrm{~Hz})$, $3.62(1 \mathrm{H}, \mathrm{q}, J=8.3 \mathrm{~Hz}), 3.56(2 \mathrm{H}, \mathrm{t}, J=6.6 \mathrm{~Hz}), 3.55-3.48(3 \mathrm{H}, \mathrm{m}), 3.31(1 \mathrm{H}, \mathrm{ddd}, J=$ 11.4, 9.2, 4.4 Hz), $3.28(1 \mathrm{H}, \mathrm{d}, J=9.0 \mathrm{~Hz}), 3.30-3.16(7 \mathrm{H}, \mathrm{m}), 3.13-3.05(4 \mathrm{H}, \mathrm{m})$, $3.05-2.88(5 \mathrm{H}, \mathrm{m}), 2.88(1 \mathrm{H}, \mathrm{dd}, J=16.5,5.9 \mathrm{~Hz}), 2.48(1 \mathrm{H}, \mathrm{dt}, J=12.1,4.5 \mathrm{~Hz})$, 2.43-2.33 (2H, m), $2.34(1 \mathrm{H}, \mathrm{dd}, J=16.5,11.2 \mathrm{~Hz}), 2.30(1 \mathrm{H}, \mathrm{dt}, J=11.2,4.0 \mathrm{~Hz})$, $2.28(1 \mathrm{H}, \mathrm{dt}, J=12.1,4.0 \mathrm{~Hz}), 2.22(1 \mathrm{H}, \mathrm{dt}, J=11.8,4.3 \mathrm{~Hz}), 2.18(2 \mathrm{H}, \mathrm{dt}, J=11.9$, $4.2 \mathrm{~Hz}), 2.14-2.03(3 \mathrm{H}, \mathrm{m}), 2.02-1.93(3 \mathrm{H}, \mathrm{m}), 1.93-1.83$ (3H, m), 1.83-1.77 (2H, m), 1.77-1.62 (5H, m), 1.61-1.37 (8H, m), $1.26(3 \mathrm{H}, \mathrm{s}), 1.20(3 \mathrm{H}, \mathrm{d}, J=6.1 \mathrm{~Hz}), 1.18(3 \mathrm{H}$, s), $0.98(3 \mathrm{H}, \mathrm{d}, J=7.2 \mathrm{~Hz}), 0.974(3 \mathrm{H}, \mathrm{d}, J=7.2 \mathrm{~Hz}), 0.968(3 \mathrm{H}, \mathrm{t}, J=7.9 \mathrm{~Hz}), 0.96$ $(3 \mathrm{H}, \mathrm{t}, J=7.9 \mathrm{~Hz}), 0.87(9 \mathrm{H}, \mathrm{s}), 0.88(1 \mathrm{H}$, septet, $J=7.9 \mathrm{~Hz}), 0.66-0.56(4 \mathrm{H}, \mathrm{m}), 0.061$ $(3 \mathrm{H}, \mathrm{s}), 0.059(3 \mathrm{H}, \mathrm{s}) ;{ }^{13} \mathrm{C} \mathrm{NMR}\left(150 \mathrm{MHz}, \mathrm{CDCl}_{3}\right) \delta 206.2,138.8,138.5,128.3,128.2$, 127.6, 127.5, 127.4, 127.3, 83.6, 82.9, 82.6, 82.0, 79.9, 79.7, 79.6, 79.1, 78.7, 78.4, 78.0, 77.5, $77.0(\mathrm{x} 2), 76.8(\mathrm{x} 2), 76.04,75.99,75.7,75.65,75.63,75.4,75.1,74.4,73.0,72.3$, 72.1, 70.6, 70.4, 70.2, 69.9, 67.2, 48.9, 44.60, 44.57, 39.2, 38.1, 37.6, 37.5, 36.3, 36.2, 35.2 (x2), 33.0, 32.5, 29.54, 29.47, 25.7, 25.2, 25.1, 18.2, 17.9, 17.31, 17.30, 16.6, 16.2, 13.0, 7.12, 7.10, 4.1, 3.9, -4.2, -4.8; HRFABMS $m / z$ calcd for $\mathrm{C}_{78} \mathrm{H}_{118} \mathrm{O}_{18} \mathrm{Si}_{2} \mathrm{Na}\left(\mathrm{MNa}^{+}\right)$ 1421.7754 , found 1421.7771 .
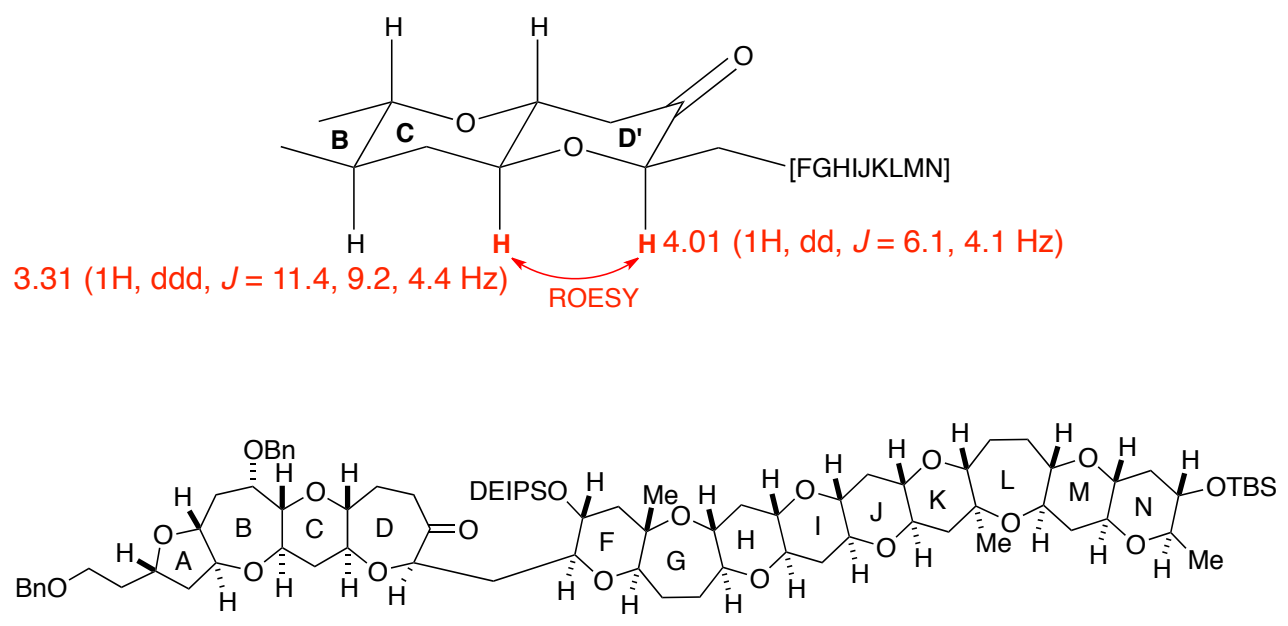

26

ABCD-FGHIJKLMN seven-membered ketone 26. To a suspension of 6-membered ketone $25(81 \mathrm{mg}, 0.058 \mathrm{mmol})$ and $\mathrm{MS} 4 \AA$ (300 $\mathrm{mg}, 50 \mathrm{mg}$ per $1 \mathrm{~mL}$ of solvent) in $\mathrm{CH}_{2} \mathrm{Cl}_{2}(6 \mathrm{~mL})$ at $-80{ }^{\circ} \mathrm{C}$ were added $\mathrm{BF}_{3} \cdot \mathrm{OEt}_{2}(0.037 \mathrm{~mL}, 0.29 \mathrm{mmol})$ and $\mathrm{TMSCH}_{2} \mathrm{~N}_{2}$ $(0.145 \mathrm{~mL}$ of a $2.0 \mathrm{M}$ solution in hexane, $0.29 \mathrm{mmol})$, and the reaction mixture was 
stirred at $-80{ }^{\circ} \mathrm{C}$ for $2 \mathrm{~h}$. The reaction was quenched with saturated aqueous $\mathrm{NaHCO}_{3}$ solution, and the reaction mixture was extracted with EtOAc. The extract was washed with water and brine, dried, and concentrated under reduced pressure to give the crude product $(99 \mathrm{mg})$. A mixture of the crude product and PPTS $(15 \mathrm{mg}, 0.058 \mathrm{mmol})$ in $\mathrm{CH}_{2} \mathrm{Cl}_{2}(1.75 \mathrm{~mL})$ and $\mathrm{MeOH}(1.75 \mathrm{~mL})$ was stirred at room temperature for $75 \mathrm{~min}$. The reaction was quenched with $\mathrm{Et}_{3} \mathrm{~N}(0.3 \mathrm{~mL})$, and the reaction mixture was concentrated under reduced pressure. Purification by flash chromatography $(30 \rightarrow 40 \%$ EtOAc in hexane) afforded ketone 26 (61 mg, 75\%) as a colorless solid. Mp $131-133{ }^{\circ} \mathrm{C} ;[\alpha]^{24}{ }_{\mathrm{D}}+25.5\left(c 1.32, \mathrm{CHCl}_{3}\right)$; IR $\left(\mathrm{CHCl}_{3}\right) 2949,2873,1716,1082 \mathrm{~cm}^{-1} ;{ }^{1} \mathrm{H}$ NMR (600 MHz, $\left.\mathrm{CDCl}_{3}\right)$ d 7.35-7.29 (8H, m), 7.28-7.24 (2H, m), 4.61 and 4.57 (each $1 \mathrm{H}, \mathrm{d}, J=12.1 \mathrm{~Hz}), 4.49$ and 4.46 (each $1 \mathrm{H}, \mathrm{d}, J=11.9 \mathrm{~Hz}), 4.25(1 \mathrm{H}, \mathrm{ddd}, J=8.8,7.8$, $7.0 \mathrm{~Hz}), 4.14(1 \mathrm{H}$, quintet, $J=6.9 \mathrm{~Hz}), 3.99(1 \mathrm{H}, \mathrm{dd}, J=6.4,4.6 \mathrm{~Hz}), 3.94(1 \mathrm{H}, \mathrm{d}, J=$ $6.4 \mathrm{~Hz}), 3.91(1 \mathrm{H}, \mathrm{ddd}, J=11.7,9.2,5.0 \mathrm{~Hz}), 3.59(1 \mathrm{H}, \mathrm{q}, J=8.1 \mathrm{~Hz}), 3.55(2 \mathrm{H}, \mathrm{t}, J=$ $6.6 \mathrm{~Hz}), 3.55-3.47(2 \mathrm{H}, \mathrm{m}), 3.41(1 \mathrm{H}, \mathrm{ddd}, J=10.8,9.2,4.8 \mathrm{~Hz}), 3.29(1 \mathrm{H}, \mathrm{ddd}, J=$ 10.6, 8.8, 4.6 Hz), 3.25-3.16 (7H, m), 3.13-3.05 (4H, m), 3.04-2.92 (5H, m), $2.91(1 \mathrm{H}$, ddd, $J=11.6,9.0,4.2 \mathrm{~Hz}), 2.85(1 \mathrm{H}$, ddd, $J=13.7,12.1,1.6 \mathrm{~Hz}), 2.40-2.34(2 \mathrm{H}, \mathrm{m})$, 2.32-2.26 (3H, m), $2.22(1 \mathrm{H}, \mathrm{dt}, J=11.7,4.2 \mathrm{~Hz}), 2.22-2.15(3 \mathrm{H}, \mathrm{m}), 2.14-2.06(3 \mathrm{H}$, m), $2.05(1 \mathrm{H}, \mathrm{dd}, J=12.3,4.6 \mathrm{~Hz}), 1.98(1 \mathrm{H}, \mathrm{dd}, J=12.3,4.6 \mathrm{~Hz}), 1.98-1.91(2 \mathrm{H}, \mathrm{m})$, $1.90-1.83(3 \mathrm{H}, \mathrm{m}), 1.82-1.63(7 \mathrm{H}, \mathrm{m}), 1.60(1 \mathrm{H}, \mathrm{q}, J=11.7 \mathrm{~Hz}), 1.53(1 \mathrm{H}, \mathrm{t}, J=12.3$ $\mathrm{Hz}), 1.51(1 \mathrm{H}, \mathrm{t}, J=12.3 \mathrm{~Hz}), 1.49-1.36(6 \mathrm{H}, \mathrm{m}), 1.26(3 \mathrm{H}, \mathrm{s}), 1.20(3 \mathrm{H}, \mathrm{d}, J=6.1 \mathrm{~Hz})$, $1.17(3 \mathrm{H}, \mathrm{s}), 0.97(3 \mathrm{H}, \mathrm{d}, J=7.2 \mathrm{~Hz}), 0.96(3 \mathrm{H}, \mathrm{d}, J=7.2 \mathrm{~Hz}), 0.96(6 \mathrm{H}, \mathrm{t}, J=7.9 \mathrm{~Hz})$, $0.88(1 \mathrm{H}, \mathrm{spt}, J=7.2 \mathrm{~Hz}), 0.87(9 \mathrm{H}, \mathrm{s}), 0.66-0.55(4 \mathrm{H}, \mathrm{m}), 0.060(3 \mathrm{H}, \mathrm{s}), 0.058(3 \mathrm{H}, \mathrm{s})$; ${ }^{13} \mathrm{C} \mathrm{NMR}\left(150 \mathrm{MHz}, \mathrm{CDCl}_{3}\right) \delta 215.7,138.8,138.4,128.3,128.1,127.5,127.45,127.35$, $127.3,83.9,83.6,82.7,82.6,82.1,80.14,80.11,79.9,79.5,78.8,78.4,78.2,77.8,77.4$, 77.0, 76.9, 76.73, 76.72, 76.0, 75.9, 75.7, 75.6, 75.3, 75.0, 72.9, 72.3, 72.1, 70.7, 70.1, 69.9, 69.8, 67.2 , 48.8, 44.6, 39.1, 38.1, 37.9, 37.6, 37.5, 36.5, 36.3, 35.8, 35.1 (x2), 33.1, 29.5, 29.44, 29.42, 25.7, 25.1, 24.9, 18.1, 17.9, 17.2 (x2), 16.5, 16.3, 12.9, 7.1, 7.0, 4.1, $3.8,-4.2,-4.8$; HRFABMS $m / z$ calcd for $\mathrm{C}_{79} \mathrm{H}_{120} \mathrm{O}_{18} \mathrm{Si}_{2} \mathrm{Na}\left(\mathrm{MNa}^{+}\right)$1435.7911, found 1435.7889. 


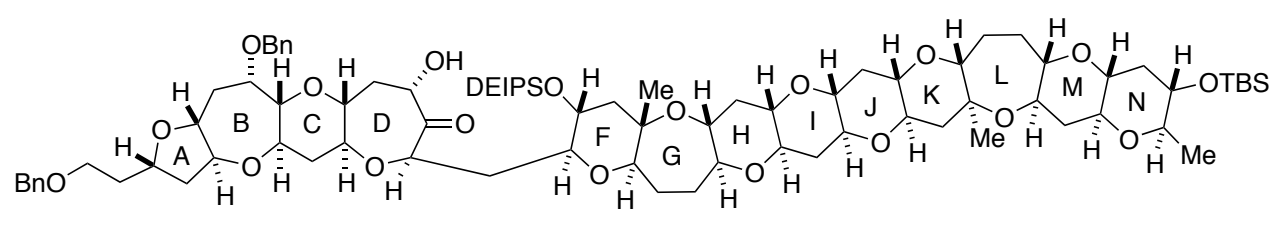

27

ABCD-FGHIJKLMN hydroxyl ketone 27. To a stirred solution of ketone 26 (48.6 $\mathrm{mg}, 0.0344 \mathrm{mmol})$ in THF $(10 \mathrm{~mL})$ at $-80{ }^{\circ} \mathrm{C}$ were added $\mathrm{Et}_{3} \mathrm{~N}(0.024 \mathrm{~mL}, 0.17 \mathrm{mmol})$, TMSCl $(0.022 \mathrm{~mL}, 0.17 \mathrm{mmol})$, and LiHMDS $(0.103 \mathrm{~mL}$ of a $1.0 \mathrm{M}$ solution in THF, $0.103 \mathrm{mmol}$ ). The reaction mixture was stirred at $-80{ }^{\circ} \mathrm{C}$ for $5 \mathrm{~min}$ and warmed to $-60{ }^{\circ} \mathrm{C}$ over $1 \mathrm{~h}$. The reaction was quenched with phosphate buffer $(\mathrm{pH} 7)$ and the reaction mixture was extracted with EtOAc. The extract was washed with brine, dried, and concentrated under reduced pressure to afford crude TMS enol ether (63 mg).

To a solution of the above TMS enol ether and $N$-methylmorpholine $N$-oxide (NMO) (403 mg, $3.448 \mathrm{mmol})$ in THF $(0.8 \mathrm{~mL})$ and water $(0.2 \mathrm{~mL})$ was added $\mathrm{OsO}_{4}$ $(0.035 \mathrm{~mL}$ of a $2.5 \mathrm{wt} \%$ solution in $t-\mathrm{BuOH}, 0.0034 \mathrm{mmol})$, and the reaction mixture was stirred at room temperature for $30 \mathrm{~h}$. The reaction mixture was extracted with EtOAc. The extract was washed with water and brine, dried, and concentrated under reduced pressure. The residue was purified by flash chromatography (40\% EtOAc in hexane) to give hydroxy ketone 27 (43.6 mg, 89\% for the two steps) as a colorless solid. $\mathrm{Mp} 131-133{ }^{\circ} \mathrm{C}$; $[\alpha]^{23}{ }_{\mathrm{D}}+34.4\left(c\right.$ 0.48, $\left.\mathrm{CHCl}_{3}\right)$; IR $\left(\mathrm{CHCl}_{3}\right)$ 3482, 2950, 2847, 1716, 1455, 1082, $1068 \mathrm{~cm}^{-1} ;{ }^{1} \mathrm{H}$ NMR (600 MHz, $\left.\mathrm{CDCl}_{3}\right) \delta 7.36-7.25(10 \mathrm{H}, \mathrm{m}), 4.63$ and 4.58 (each $1 \mathrm{H}, \mathrm{d}, J=12.1 \mathrm{~Hz}), 4.55(1 \mathrm{H}$, ddd, $J=12.7,4.8,3.5 \mathrm{~Hz}), 4.51$ and 4.48 (each $1 \mathrm{H}, \mathrm{d}, J=11.9 \mathrm{~Hz}), 4.25(1 \mathrm{H}, \mathrm{td}, J=8.1,7.4 \mathrm{~Hz}), 4.19(1 \mathrm{H}, \mathrm{dd}, J=6.2,3.3 \mathrm{~Hz})$, 4.15 (1H, quintet, $J=7.0 \mathrm{~Hz}), 3.98(1 \mathrm{H}, \mathrm{d}, J=6.4 \mathrm{~Hz}), 3.93(1 \mathrm{H}, \mathrm{ddd}, J=11.4,9.0,4.9$ $\mathrm{Hz}), 3.61(1 \mathrm{H}, \mathrm{d}, J=4.8 \mathrm{~Hz}, \mathrm{OH}), 3.60(1 \mathrm{H}, \mathrm{q}, J=8.1 \mathrm{~Hz}), 3.56(2 \mathrm{H}, \mathrm{t}, J=6.5 \mathrm{~Hz})$, $3.53(1 \mathrm{H}, \mathrm{ddd}, J=10.8,9.3,5.0 \mathrm{~Hz}), 3.49(1 \mathrm{H}$, ddd, $J=10.6,9.2,5.0 \mathrm{~Hz}), 3.38(1 \mathrm{H}$, ddd, $J=11.0,9.1,4.7 \mathrm{~Hz}), 3.32(1 \mathrm{H}, \mathrm{ddd}, J=11.2,9.2,4.4 \mathrm{~Hz}), 3.30$ (1H, ddd, $J=10.8$, 8.8, 4.6 Hz), 3.25 (1H, d, $J=9.4 \mathrm{~Hz}), 3.29-3.17(5 \mathrm{H}, \mathrm{m}), 3.13-3.04$ (4H, m), 3.05-2.96 (4H, m), $2.95(1 \mathrm{H}$, ddd, $J=11.2,8.8,4.0 \mathrm{~Hz}), 2.92(1 \mathrm{H}$, ddd, $J=11.3,9.0,4.2 \mathrm{~Hz})$, 2.43-2.27 (6H, m), $2.23(1 \mathrm{H}, \mathrm{dt}, J=11.6,4.4 \mathrm{~Hz}), 2.19(1 \mathrm{H}, \mathrm{dt}, J=11.7,4.4 \mathrm{~Hz}), 2.17$ $(1 \mathrm{H}, \mathrm{dt}, J=11.7,4.4 \mathrm{~Hz}), 2.15-2.04(3 \mathrm{H}, \mathrm{m}), 2.01-1.92(3 \mathrm{H}, \mathrm{m}), 1.91-1.82(4 \mathrm{H}, \mathrm{m})$, $1.82-1.73(3 \mathrm{H}, \mathrm{m}), 1.73-1.62(2 \mathrm{H}, \mathrm{m}), 1.61(1 \mathrm{H}, \mathrm{q}, J=12.1 \mathrm{~Hz}), 1.56(1 \mathrm{H}, \mathrm{m}), 1.54$ $(1 \mathrm{H}, \mathrm{t}, J=12.6 \mathrm{~Hz}), 1.54(1 \mathrm{H}, \mathrm{q}, J=12.6 \mathrm{~Hz}), 1.51(1 \mathrm{H}, \mathrm{t}, J=11.9 \mathrm{~Hz}), 1.48-1.38(5 \mathrm{H}$, m), $1.27(3 \mathrm{H}, \mathrm{s}), 1.21(3 \mathrm{H}, \mathrm{d}, J=6.1 \mathrm{~Hz}), 1.15(3 \mathrm{H}, \mathrm{s}), 0.982(3 \mathrm{H}, \mathrm{d}, J=7.2 \mathrm{~Hz}), 0.976$ 
$(3 \mathrm{H}, \mathrm{d}, J=7.2 \mathrm{~Hz}), 0.97(6 \mathrm{H}, \mathrm{t}, J=8.0 \mathrm{~Hz}), 0.90(1 \mathrm{H}$, septet, $J=7.9 \mathrm{~Hz}), 0.88(9 \mathrm{H}, \mathrm{s})$, 0.68-0.56 (4H, m), $0.070(3 \mathrm{H}, \mathrm{s}), 0.068(3 \mathrm{H}, \mathrm{s}) ;{ }^{13} \mathrm{C} \mathrm{NMR}\left(150 \mathrm{MHz}, \mathrm{CDCl}_{3}\right) \delta 214.9$, $138.8,138.5,128.3,128.2$, 127.6, 127.5, 127.39, 127.38, 83.6, 82.85, 82.79, 82.7, 82.1, $80.6,79.9,79.6,79.0,78.4,77.73,77.66,77.6,77.5,77.04,76.99,76.8(x 2), 76.04$, $75.98,75.7,75.6,75.4,75.0,73.0,72.3,72.1,71.2,70.7,70.2,70.0$ (x2), 67.2, 48.7, 44.6, 39.2, 39.0, 38.1, 37.6 (x2), 37.5, 37.0, 36.3, 35.20, 35.19, 33.1, 29.5, 29.4, 25.7, $25.1,24.6,18.2,17.9,17.3$ (x2), 16.6, 16.3, 13.0, 7.1, 7.0, 4.1, 3.9, -4.2,-4.8; HRFABMS $m / z$ calcd for $\mathrm{C}_{79} \mathrm{H}_{120} \mathrm{O}_{19} \mathrm{Si}_{2} \mathrm{Na}\left(\mathrm{MNa}^{+}\right)$1451.7860, found 1451.7853 .

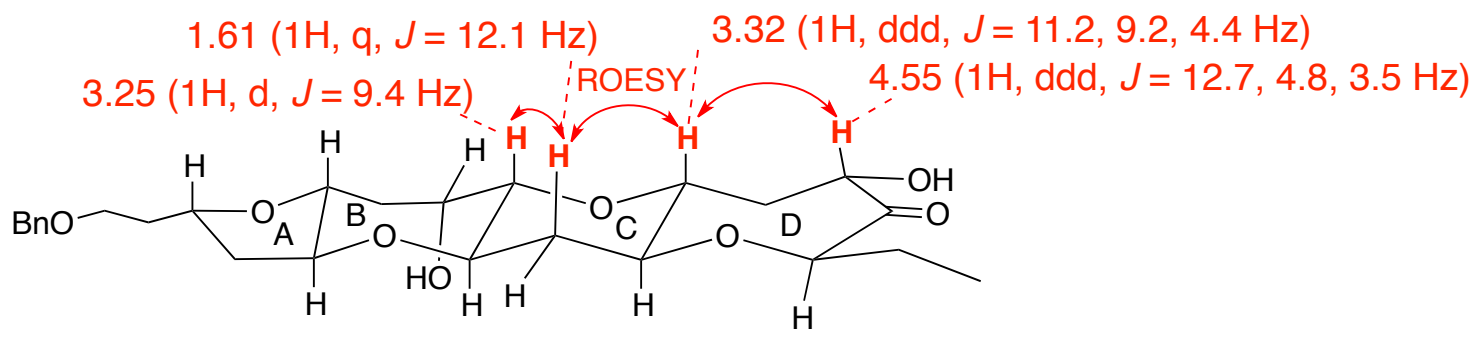

\section{ABCDEFGHIJKLMN hydroxyl acetal 28a and $28 \mathrm{~b}$.}

i) Methyl acetalization: a mixture of hydroxyl ketone 27 (45.4 $\mathrm{mg}, 0.0317 \mathrm{mmol})$ and $\mathrm{TsOH} \cdot \mathrm{H}_{2} \mathrm{O} \quad(0.050 \mathrm{~mL}, \quad 1.63 \mathrm{M}$ solution in $\mathrm{MeOH}, 0.082 \mathrm{mmol})$ in $\mathrm{CH}_{2} \mathrm{Cl}_{2} / \mathrm{CH}(\mathrm{OMe})_{3}(1: 1,1 \mathrm{~mL})$ was stirred at room temperature for $3 \mathrm{~h}$. The reaction was quenched with $\mathrm{Et}_{3} \mathrm{~N}(0.3 \mathrm{~mL})$, and the reaction mixture was concentrated under reduced pressure to give a crude methyl acetal containing a small amount of dimethyl orthoester of the D-ring hydroxyl group.

ii) Orthoester methanolysis: $\mathrm{Et}_{3} \mathrm{~N}$ in the crude acetal was completely removed by azeotropic evaporation with EtOAc. This crude acetal was dissolved in $\mathrm{CH}_{2} \mathrm{Cl}_{2} / \mathrm{MeOH}$ $(1: 1,1 \mathrm{~mL})$ and PPTS $(15.6 \mathrm{mg}, 0.062 \mathrm{mmol})$ was added. The reaction mixture was stirred at room temperature for $0.5 \mathrm{~h}$. The reaction was quenched with $\mathrm{Et}_{3} \mathrm{~N}(0.3 \mathrm{~mL})$, and the reaction mixture was concentrated under reduced pressure Purification by flash chromatography $(50 \rightarrow 60 \%$ EtOAc in hexane) afforded hydroxy methyl acetal 28a $(31.3$ $\mathrm{mg}, 75 \%$ ) and dihydroxy methyl acetal $\mathbf{2 8 b}$ (5.9 mg, 15\%).

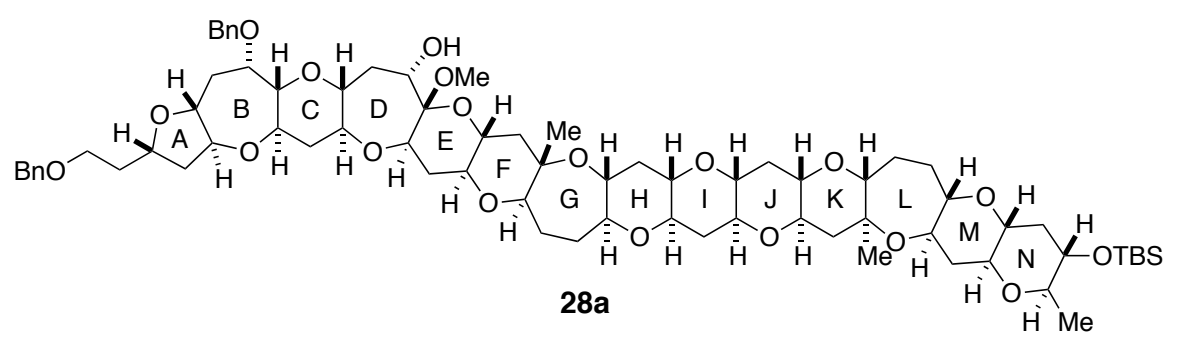


Hydroxy methyl acetal 28a. Colorless amorphous solid; $[\alpha]^{24}+51.7\left(c 0.42, \mathrm{CHCl}_{3}\right)$; IR $\left(\mathrm{CHCl}_{3}\right)$ 3512, 2948, 2874, 2856, $1066 \mathrm{~cm}^{-1} ;{ }^{1} \mathrm{H}$ NMR $\left(600 \mathrm{MHz}, \mathrm{CDCl}_{3}\right) \delta$ 7.37-7.30 (8H, m), 7.28-7.25 (2H, m), $4.63(2 \mathrm{H}, \mathrm{s}), 4.49$ and 4.46 (each $1 \mathrm{H}, \mathrm{d}, J=11.9$ $\mathrm{Hz}), 4.23(1 \mathrm{H}, \mathrm{q}, J=7.9 \mathrm{~Hz}), 4.12(1 \mathrm{H}$, quintet, $J=7.0 \mathrm{~Hz}), 4.08(1 \mathrm{H}, \mathrm{m}), 3.93(1 \mathrm{H}, \mathrm{d}$, $J=5.9 \mathrm{~Hz}), 3.91(1 \mathrm{H}, \mathrm{ddd}, J=11.6,9.0,4.8 \mathrm{~Hz}), 3.84(1 \mathrm{H}, \mathrm{dd}, J=11.9,4.4 \mathrm{~Hz})$, 3.59-3.50 (5H, m), 3.47 (1H, ddd, $J=11.7,9.3,4.6 \mathrm{~Hz}), 3.29(3 \mathrm{H}, \mathrm{s}), 3.33-3.16(8 \mathrm{H}$, $\mathrm{m}), 3.14(1 \mathrm{H}, \mathrm{d}, J=9.0 \mathrm{~Hz}), 3.12-3.06(3 \mathrm{H}, \mathrm{m}), 3.05-2.95(5 \mathrm{H}, \mathrm{m}), 2.92(1 \mathrm{H}, \mathrm{ddd}, J=$ $11.3,9.0,4.2 \mathrm{~Hz}), 2.38-2.32(4 \mathrm{H}, \mathrm{m}), 2.30(1 \mathrm{H}, \mathrm{dt}, J=11.0,3.7 \mathrm{~Hz}), 2.29(1 \mathrm{H}, \mathrm{dt}, J=$ 11.0, 3.7 Hz), $2.22(1 \mathrm{H}, \mathrm{dt}, J=11.5,4.0 \mathrm{~Hz}), 2.22-2.16(2 \mathrm{H}, \mathrm{m}), 2.14-2.08(3 \mathrm{H}, \mathrm{m})$, $2.06(1 \mathrm{H}, \mathrm{dd}, J=12.0,4.1 \mathrm{~Hz}), 1.98(1 \mathrm{H}, \mathrm{dd}, J=11.9,4.2 \mathrm{~Hz}), 1.96-1.90(2 \mathrm{H}, \mathrm{m})$, 1.90-1.81 (6H, m), 1.81-1.64 (5H, m), 1.59-1.50 (3H, m), 1.49-1.38 (5H, m), $1.27(3 \mathrm{H}$, s), $1.26(3 \mathrm{H}, \mathrm{s}), 1.20(3 \mathrm{H}, \mathrm{d}, J=6.1 \mathrm{~Hz}), 0.87(9 \mathrm{H}, \mathrm{s}), 0.06(6 \mathrm{H}, \mathrm{s}) ;{ }^{13} \mathrm{C} \mathrm{NMR}(150 \mathrm{MHz}$, $\left.\mathrm{CDCl}_{3}\right) \delta 139.0,138.5,128.3,128.2,127.6,127.53,127.48,127.3,99.7,83.6,83.5$, 82.7, 82.1, 79.8, 79.6, 78.9, 78.4 (x2), 78.3, 77.7, 77.5, 77.3, 77.02, 77.01, 76.8, 76.7, 76.04, 75.98, 75.8, 75.7, 75.6, 75.5, 75.0, 73.0, 72.3, 72.1, 70.8, 70.20, 70.17, 70.0, 68.8, 67.3, 48.1, 44.6, 44.1, 39.2, 38.1, 37.9, 37.6, 37.5, 36.4, 36.3, 35.19, 35.16, 33.3, 32.1, 29.5, 29.3, 25.7, 25.1, 25.0, 18.2, 17.9, 16.7, 16.6, -4.2, -4.8; HRFABMS $m / z$ calcd for $\mathrm{C}_{73} \mathrm{H}_{106} \mathrm{O}_{19} \mathrm{SiNa}\left(\mathrm{MNa}^{+}\right)$1337.6995, found 1337.6992.
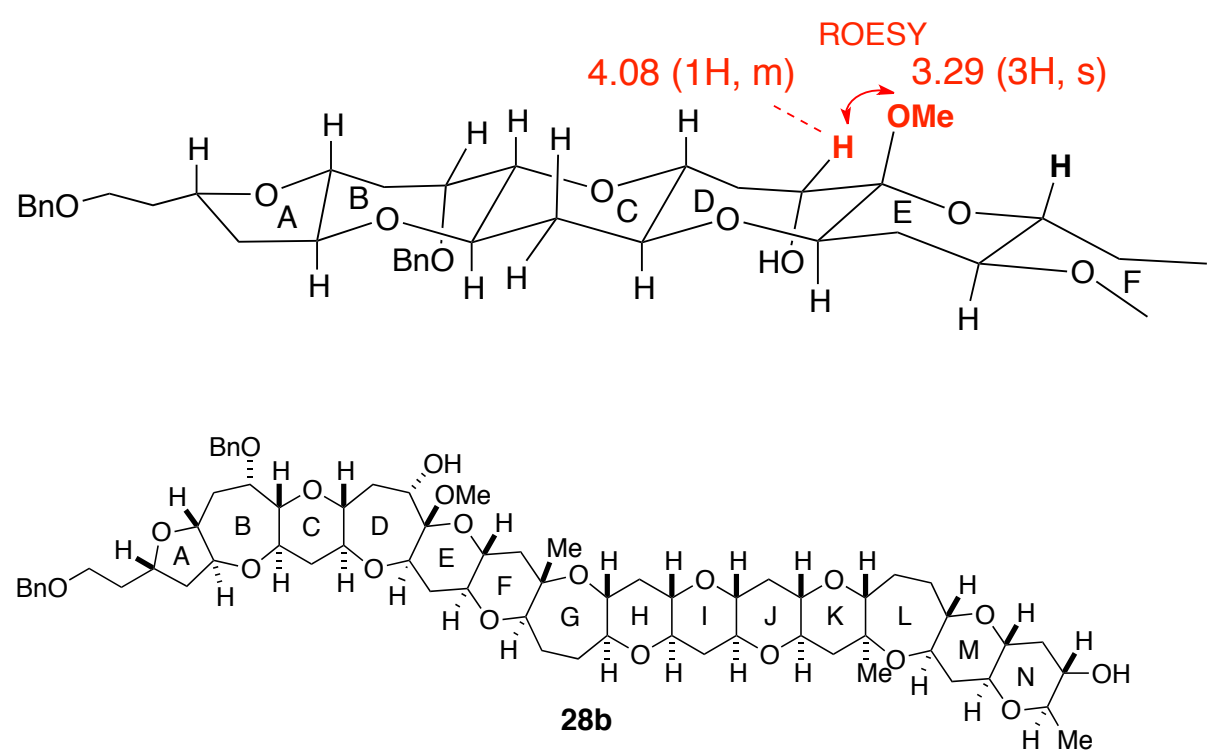

Dihydroxy methyl acetal 28b. Colorless solid; mp 270-273 ${ }^{\circ} \mathrm{C} ;[\alpha]^{28}{ }_{\mathrm{D}}+49.1(c 0.49$, $\left.\mathrm{CHCl}_{3}\right)$; IR $\left(\mathrm{CHCl}_{3}\right)$ 3480, 2946, 2873, $1064 \mathrm{~cm}^{-1} ;{ }^{1} \mathrm{H} \mathrm{NMR}\left(600 \mathrm{MHz}, \mathrm{CDCl}_{3}\right) \delta$ 7.37-7.30 (8H, m), 7.28-7.25 (2H, m), $4.63(2 \mathrm{H}, \mathrm{s}), 4.49$ and 4.46 (each 1H, d, $J=11.9$ $\mathrm{Hz}), 4.23(1 \mathrm{H}, \mathrm{q}, J=7.9 \mathrm{~Hz}), 4.12(1 \mathrm{H}$, quintet, $J=7.0 \mathrm{~Hz}), 4.08(1 \mathrm{H}, \mathrm{m}), 3.93(1 \mathrm{H}, \mathrm{d}$, 
$J=5.9 \mathrm{~Hz}), 3.91(1 \mathrm{H}, \mathrm{ddd}, J=11.6,9.0,4.8 \mathrm{~Hz}), 3.84(1 \mathrm{H}, \mathrm{dd}, J=11.9,4.4 \mathrm{~Hz})$, 3.59-3.50 (5H, m), $3.47(1 \mathrm{H}, \mathrm{ddd}, J=11.7,9.3,4.6 \mathrm{~Hz}), 3.29(3 \mathrm{H}, \mathrm{s}), 3.34-3.21(8 \mathrm{H}$, $\mathrm{m}), 3.14(1 \mathrm{H}, \mathrm{d}, J=9.0 \mathrm{~Hz}), 3.12-3.06(3 \mathrm{H}, \mathrm{m}), 3.05-2.93(6 \mathrm{H}, \mathrm{m}), 2.38-2.27(7 \mathrm{H}, \mathrm{m})$, 2.20 (1H, dt, $J=12.0,4.1 \mathrm{~Hz}), 2.19(1 \mathrm{H}, \mathrm{dt}, J=12.0,4.1 \mathrm{~Hz}), 2.14-2.08(3 \mathrm{H}, \mathrm{m}), 2.06$ $(1 \mathrm{H}, \mathrm{dd}, J=11.9,4.2 \mathrm{~Hz}), 1.98(1 \mathrm{H}, \mathrm{dd}, J=11.9,4.2 \mathrm{~Hz}), 1.96-1.90(2 \mathrm{H}, \mathrm{m})$, 1.90-1.82 (6H, m), 1.81-1.65 (5H, m), 1.58-1.50 (4H, m), 1.49-1.35 (5H, m), $1.28(3 \mathrm{H}$, $\mathrm{d}, J=6.2 \mathrm{~Hz}), 1.27(3 \mathrm{H}, \mathrm{s}), 1.26(3 \mathrm{H}, \mathrm{s}) ;{ }^{13} \mathrm{C} \mathrm{NMR}\left(150 \mathrm{MHz}, \mathrm{CDCl}_{3}\right) \delta 139.0,138.4$, $128.3,128.2$, 127.6, 127.53, 127.48, 127.3, 99.7, 83.6, 83.5, 82.7, 82.1, 79.8, 79.6, 78.8, 78.4, 78.3, 78.1, 77.7, 77.5, 77.3, 77.03, 77.01, 76.8, 76.7, 76.1, 76.0, 75.8, 75.7, 75.6, 75.5, 75.0, 73.0, 72.3, 71.5, 70.8, 70.18, 70.15, 70.0, 68.8, 67.3, 48.1, 44.6, 44.1, 38.6, 38.1, 37.9, 37.5, 37.4, 36.4, 36.3, 35.2, 35.1, 33.3, 32.1, 29.4, 29.3, 25.1, 25.0, 17.8, 16.7, 16.6; HRFABMS $m / z$ calcd for $\mathrm{C}_{67} \mathrm{H}_{92} \mathrm{O}_{19} \mathrm{Na}\left(\mathrm{MNa}^{+}\right)$1223.6131, found 1223.6144.

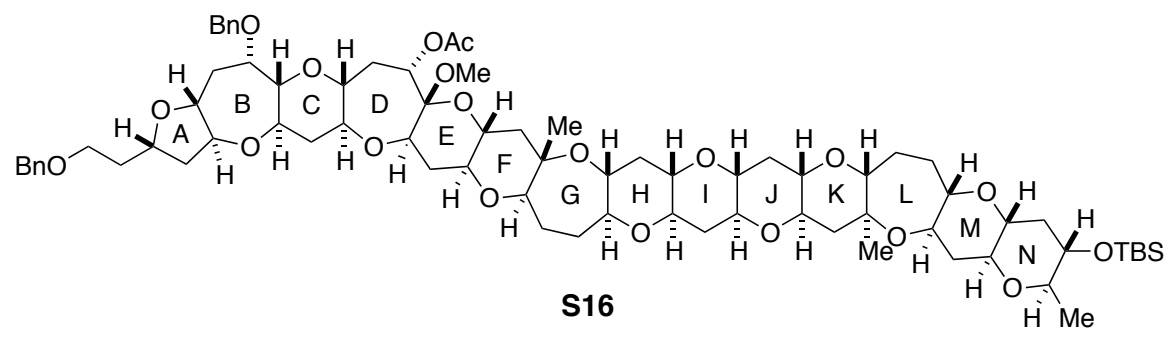

ABCDEFGHIJKLMN acetoxy acetal S16. To a solution of hydroxyl acetal 28a (31.3 $\mathrm{mg}, 0.0238 \mathrm{mmol})$ in pyridine $(1 \mathrm{~mL})$ and acetic anhydride $(1 \mathrm{~mL})$ was added $N, N$-dimethylaminopyridine $(1.0 \mathrm{mg}, 0.0082 \mathrm{mmol})$. The reaction mixture was stirred at room temperature for $2 \mathrm{~h}$, and then concentrated under reduced pressure. Purification by flash chromatography (50\% EtOAc in hexane) afforded acetylated acetal S16 (31.2 mg, 97\%) as a colorless amorphous solid; $[\alpha]^{22}{ }_{\mathrm{D}}+53.5\left(c\right.$ 0.38, $\left.\mathrm{CHCl}_{3}\right)$; IR $\left(\mathrm{CHCl}_{3}\right) 2949$, 2873, 2857, 1746, $1068 \mathrm{~cm}^{-1}$; ${ }^{1} \mathrm{H}$ NMR (600 MHz, $\left.\mathrm{CDCl}_{3}\right) \delta 7.36-7.29$ (8H, m), 7.29-7.23 (2H, m), $5.25(1 \mathrm{H}, \mathrm{t}, J=4.0 \mathrm{~Hz}), 4.61$ and 4.58 (each $1 \mathrm{H}, \mathrm{d}, J=12.3 \mathrm{~Hz}$ ), 4.49 and 4.46 (each $1 \mathrm{H}, \mathrm{d}, J=11.9 \mathrm{~Hz}), 4.22(1 \mathrm{H}$, ddd, $J=8.6,8.0,7.2 \mathrm{~Hz}), 4.12(1 \mathrm{H}$, quintet, $J=7.0 \mathrm{~Hz}), 3.92(1 \mathrm{H}, \mathrm{ddd}, J=11.7,9.0,5.0 \mathrm{~Hz}), 3.90(1 \mathrm{H}, \mathrm{d}, J=6.2 \mathrm{~Hz}), 3.87$ $(1 \mathrm{H}, \mathrm{dd}, J=11.9,4.4 \mathrm{~Hz}), 3.61-3.50(6 \mathrm{H}, \mathrm{m}), 3.40(1 \mathrm{H}, \mathrm{td}, J=9.0,4.1 \mathrm{~Hz}), 3.29(3 \mathrm{H}$, s), $3.29(1 \mathrm{H}, \mathrm{ddd}, J=10.6,9.0,4.4 \mathrm{~Hz}), 3.25-3.16(6 \mathrm{H}, \mathrm{m}), 3.12(1 \mathrm{H}, \mathrm{d}, J=9.0 \mathrm{~Hz})$, 3.12-3.05 (3H, m), 3.05-2.93 (5H, m), $2.91(1 \mathrm{H}, \mathrm{ddd}, J=11.7,9.0,4.2 \mathrm{~Hz}), 2.43(1 \mathrm{H}$, ddd, $J=16.6,8.8,4.2 \mathrm{~Hz}), 2.38(1 \mathrm{H}, \mathrm{dt}, J=12.2,4.6 \mathrm{~Hz}), 2.34(1 \mathrm{H}, \mathrm{dd}, J=14.1,6.2$ 
Hz), $2.30(1 \mathrm{H}, \mathrm{dt}, J=11.4,3.7 \mathrm{~Hz}), 2.28(1 \mathrm{H}, \mathrm{dt}, J=11.4,3.7 \mathrm{~Hz}), 2.22(1 \mathrm{H}, \mathrm{dt}, J=$ 11.6, 4.2 Hz), 2.21-2.16 (2H, m), 2.14-2.07 (3H, m), 2.05 (1H, dd, $J=12.1,4.4 \mathrm{~Hz})$, $1.98(3 \mathrm{H}, \mathrm{s}), 1.96-1.91(3 \mathrm{H}, \mathrm{m}), 1.90-1.65(11 \mathrm{H}, \mathrm{m}), 1.55(1 \mathrm{H}, \mathrm{q}, J=12.1 \mathrm{~Hz}), 1.53$ $(1 \mathrm{H}, \mathrm{t}, J=12.4 \mathrm{~Hz}), 1.50(1 \mathrm{H}, \mathrm{t}, J=12.4 \mathrm{~Hz}), 1.47-1.37(5 \mathrm{H}, \mathrm{m}), 1.26(3 \mathrm{H}, \mathrm{s}), 1.25$ $(3 \mathrm{H}, \mathrm{s}), 1.20(3 \mathrm{H}, \mathrm{d}, J=6.2 \mathrm{~Hz}), 0.87(9 \mathrm{H}, \mathrm{s}), 0.060(3 \mathrm{H}, \mathrm{s}), 0.058(3 \mathrm{H}, \mathrm{s}) ;{ }^{13} \mathrm{C} \mathrm{NMR}$ $\left(150 \mathrm{MHz}, \mathrm{CDCl}_{3}\right) \delta 169.5,138.8,138.5,128.3,128.2,127.6,127.5,127.4,127.2,99.0$, 83.6, 83.4, 82.8, 82.1, 79.8, 79.6, 78.9, 78.4 (x2), 77.55, 77.47, 77.2 (x2), 77.0 (x2), $76.8,76.7,76.3,76.04,75.98,75.8,75.7,75.6,75.0,73.0,72.1,72.0,71.4,70.8,70.23$, 70.16, 68.8, 67.3, 48.1, 44.6, 44.2, 39.2, 38.4, 38.2, 37.6, 37.4, 36.3, 35.19, 35.16, 33.01, $32.95,31.9,29.5,29.3,25.7,25.1,25.0,21.2,18.2,17.9,16.7,16.6,-4.2,-4.8$; HRFABMS $m / z$ calcd for $\mathrm{C}_{75} \mathrm{H}_{108} \mathrm{O}_{20} \mathrm{SiNa}\left(\mathrm{MNa}^{+}\right)$1379.7101, found 1379.7103.

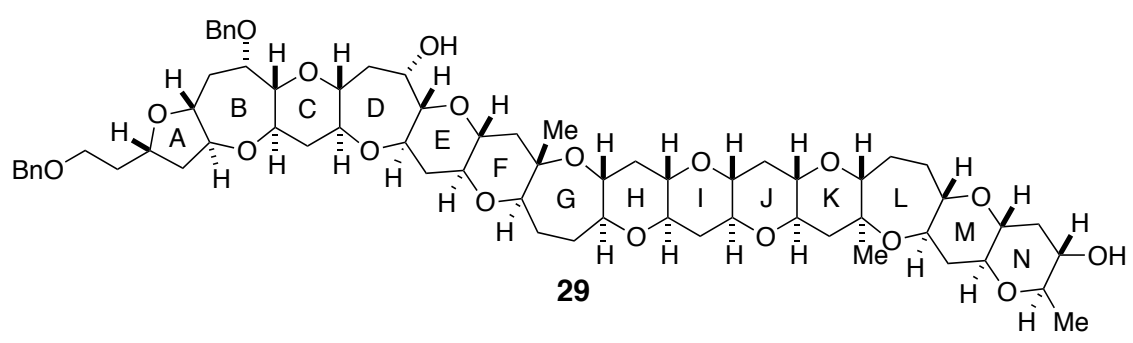

Tetradecacyclic diol 29. To a solution of methyl acetal S16 (64 mg, $0.023 \mathrm{mmol})$ in $\mathrm{CH}_{2} \mathrm{Cl}_{2}(2.5 \mathrm{~mL})$ at $-20{ }^{\circ} \mathrm{C}$ were added $\mathrm{Et}_{3} \mathrm{SiH}(0.074 \mathrm{~mL}, 0.46 \mathrm{mmol})$ and TMSOTf $(0.017 \mathrm{~mL}, 0.092 \mathrm{mmol})$, and the reaction mixture was stirred at $-20^{\circ} \mathrm{C}$ for $15 \mathrm{~h}$. The reaction was quenched with saturated aqueous $\mathrm{NaHCO}_{3}$ solution, and the reaction mixture was extracted with EtOAc. The extract was washed with brine, dried, and concentrated under reduced pressure to afford crude tetradecacyclic acetate $(32.7 \mathrm{mg})$.

A mixture of the above crude tetradecacyclic acetate and $\mathrm{NaOH}(1.0 \mathrm{~mL}, 2.5 \mathrm{M}$ solution in $\mathrm{MeOH}, 2.5 \mathrm{mmol})$ in THF $(1.5 \mathrm{~mL})$ was stirred at room temperature for 2.5 h. The reaction was quenched by saturated aqueous $\mathrm{NH}_{4} \mathrm{Cl}$ solution and the reaction mixture was extracted with EtOAc. The extract was washed with brine, dried, and concentrated under reduced pressure. Purification by flash chromatography $(100 \%$ EtOAc) afforded tetradecacyclic diol $29(23.4 \mathrm{mg}, 87 \%)$ as a colorless solid. Mp 268-271 ${ }^{\circ} \mathrm{C} ;[\alpha]^{25}{ }_{\mathrm{D}}+27.5\left(c 0.32, \mathrm{CHCl}_{3}\right)$; IR $\left(\mathrm{CHCl}_{3}\right)$ 3447, 2933, 2873, 2856, 1746, $1068 \mathrm{~cm}^{-1} ;{ }^{1} \mathrm{H}$ NMR $\left(600 \mathrm{MHz}, \mathrm{CDCl}_{3}\right) \delta$ 7.37-7.30 (8H, m), 7.28-7.25 (2H, m), 4.64 $(2 \mathrm{H}, \mathrm{s}), 4.49$ and 4.46 (each $1 \mathrm{H}, \mathrm{d}, J=11.9 \mathrm{~Hz}), 4.22(1 \mathrm{H}, \mathrm{ddd}, J=8.9,7.8,6.9 \mathrm{~Hz}$ ), $4.19(1 \mathrm{H}, \mathrm{ddd}, J=6.4,4.0,2.4 \mathrm{~Hz}), 4.12(1 \mathrm{H}$, quintet, $J=6.9 \mathrm{~Hz}), 3.94(1 \mathrm{H}, \mathrm{d}, J=5.5$ 
Hz), $3.93(1 \mathrm{H}, \mathrm{ddd}, J=11.6,8.6,5.0 \mathrm{~Hz}), 3.73(1 \mathrm{H}, \mathrm{ddd}, J=11.1,9.4,4.7 \mathrm{~Hz}), 3.62$ $(1 \mathrm{H}, \mathrm{ddd}, J=11.6,9.4,4.0 \mathrm{~Hz}), 3.57(1 \mathrm{H}, \mathrm{q}, J=8.5 \mathrm{~Hz}), 3.54(2 \mathrm{H}, \mathrm{t}, J=6.5 \mathrm{~Hz})$, 3.55-3.51 (2H, m), 3.32 (1H, ddd, $J=10.9,9.3,4.8 \mathrm{~Hz}), 3.26-3.21(2 \mathrm{H}, \mathrm{m}), 3.21-3.11$ (6H, m), 3.11-3.05 (4H, m), 3.05-2.92 (6H, m), 2.39-2.27 (8H, m), 2.22-2.16 (2H, m), 2.15-2.08 (2H, m), $2.05(1 \mathrm{H}, \mathrm{dd}, J=11.9,4.4 \mathrm{~Hz}), 2.03(1 \mathrm{H}, \mathrm{dd}, J=12.3,4.4 \mathrm{~Hz})$, 1.98-1.90 (3H, m), 1.89-1.82 (4H, m), 1.81-1.65 (6H, m), $1.53(1 \mathrm{H}, \mathrm{t}, J=11.9 \mathrm{~Hz})$, $1.52(1 \mathrm{H}, \mathrm{t}, J=11.9 \mathrm{~Hz}), 1.48(1 \mathrm{H}, \mathrm{q}, J=11.2 \mathrm{~Hz}), 1.48-1.39(5 \mathrm{H}, \mathrm{m}), 1.38(1 \mathrm{H}, \mathrm{q}, J=$ $11.2 \mathrm{~Hz}), 1.28(3 \mathrm{H}, \mathrm{d}, J=6.1 \mathrm{~Hz}), 1.26(3 \mathrm{H}, \mathrm{s}), 1.25(3 \mathrm{H}, \mathrm{s}) ;{ }^{13} \mathrm{C}$ NMR $(150 \mathrm{MHz}$, $\left.\mathrm{CDCl}_{3}\right) \delta 138.9,138.5,128.3,128.2,127.60,127.57,127.5,127.3,83.6,83.4,83.3$, 82.9, 82.1, 79.8, 79.6, 78.9, 78.7, 78.1, 77.7, 77.51, 77.47, 77.03 (x2), 77.0, 76.8, 76.7, 76.4, 76.1, 76.0, 75.72, 75.69, 75.66, 75.0, 73.2, 73.0, 72.3, 71.5, 70.8, 70.2, 70.1, 69.8, 67.2, 44.6 (x2), 38.6, 38.3, 38.1, 37.5, 37.4, 36.8, 36.6, 36.3, 35.18, 35.16, 33.4, 29.4, 29.3, 25.08, 25.06, 17.8, 16.6 (x2); HRFABMS $m / z$ calcd for $\mathrm{C}_{66} \mathrm{H}_{90} \mathrm{O}_{18} \mathrm{Na}\left(\mathrm{MNa}^{+}\right)$ 1193.6025, found 1193.6010 .

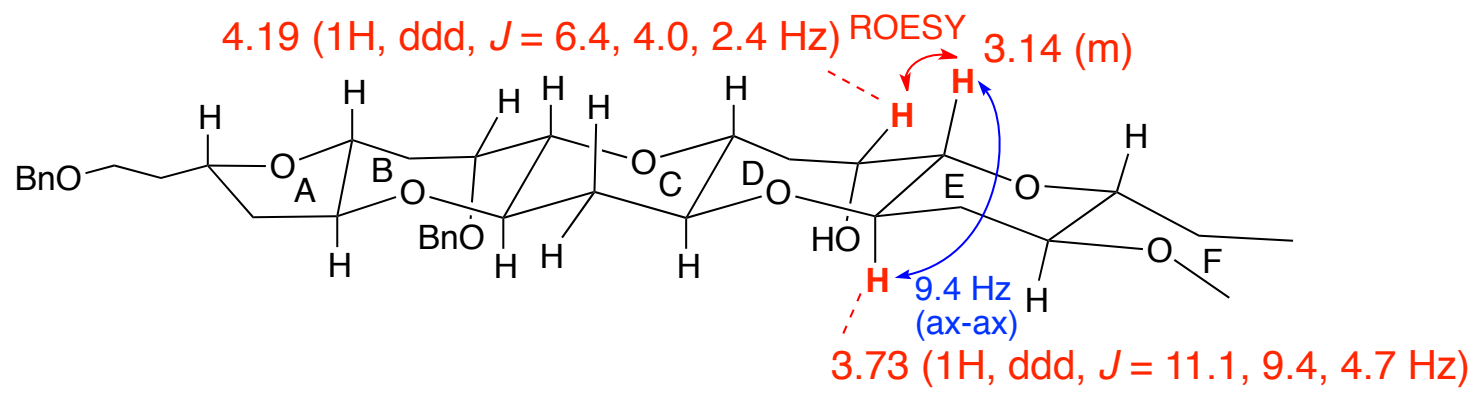

Tetradecacyclic diol 29 from dihydroxyl acetal $28 \mathrm{~b}$.



Diacetyled product S17. To a solution of dihydroxy acetal $\mathbf{2 8 b}(5.9 \mathrm{mg}, 0.0049 \mathrm{mmol})$ in pyridine $(0.5 \mathrm{~mL})$ and acetic anhydride $(0.5 \mathrm{~mL})$ was added $\mathrm{N}, \mathrm{N}$-dimethylaminopyridine $(1.0 \mathrm{mg}, 0.0082 \mathrm{mmol})$. The reaction mixture was stirred at room temperature for $7 \mathrm{~h}$, and then concentrated under reduced pressure. Purification by flash chromatography $(50 \% \rightarrow 70 \%$ EtOAc in hexane) afforded acetylated acetal S17 (6.3 mg, quant.) as a colorless amorphous solid. $[\alpha]^{27}+50.4\left(c 0.53, \mathrm{CHCl}_{3}\right)$; IR 
$\left(\mathrm{CHCl}_{3}\right)$ 2946, 2874, 1742, 1238, $1066 \mathrm{~cm}^{-1} ;{ }^{1} \mathrm{H}$ NMR (600 MHz, $\left.\mathrm{CDCl}_{3}\right) \delta$ 7.36-7.29 $(8 \mathrm{H}, \mathrm{m}), 7.29-7.23(2 \mathrm{H}, \mathrm{m}), 5.25(1 \mathrm{H}, \mathrm{t}, J=4.0 \mathrm{~Hz}), 4.61$ and 4.58 (each $1 \mathrm{H}, \mathrm{d}, J=$ $12.3 \mathrm{~Hz}), 4.51(1 \mathrm{H}, \mathrm{ddd}, J=11.2,9.5,4.8 \mathrm{~Hz}), 4.49$ and 4.46 (each $1 \mathrm{H}, \mathrm{d}, J=11.9 \mathrm{~Hz})$, $4.22(1 \mathrm{H}, \mathrm{ddd}, J=8.6,8.0,7.2 \mathrm{~Hz}), 4.12(1 \mathrm{H}$, quintet, $J=7.0 \mathrm{~Hz}), 3.92$ (1H, ddd, $J=$ 11.7, 9.0, $5.0 \mathrm{~Hz}), 3.90(1 \mathrm{H}, \mathrm{d}, J=6.2 \mathrm{~Hz}), 3.87(1 \mathrm{H}, \mathrm{dd}, J=11.9,4.4 \mathrm{~Hz}), 3.61-3.50$ $(6 \mathrm{H}, \mathrm{m}), 3.42-3.36(2 \mathrm{H}, \mathrm{m}), 3.29(3 \mathrm{H}, \mathrm{s}), 3.27-3.17(5 \mathrm{H}, \mathrm{m}), 3.12(1 \mathrm{H}, \mathrm{d}, J=9.0 \mathrm{~Hz})$, $3.12-3.05$ (3H, m), 3.05-2.93 (6H, m), 2.43 (1H, ddd, $J=16.6,8.8,4.2 \mathrm{~Hz}), 2.41-2.26$ $(5 \mathrm{H}, \mathrm{m}), 2.21-2.16(2 \mathrm{H}, \mathrm{m}), 2.14-2.07(3 \mathrm{H}, \mathrm{m}), 2.05(3 \mathrm{H}, \mathrm{s}), 2.05(1 \mathrm{H}, \mathrm{dd}, J=12.1,4.4$ $\mathrm{Hz}), 1.98(3 \mathrm{H}, \mathrm{s}), 1.96-1.91(3 \mathrm{H}, \mathrm{m}), 1.90-1.64(11 \mathrm{H}, \mathrm{m}), 1.55-1.36(8 \mathrm{H}, \mathrm{m}), 1.25(6 \mathrm{H}$, s), $1.17(3 \mathrm{H}, \mathrm{d}, J=6.2 \mathrm{~Hz}) ;{ }^{13} \mathrm{C} \mathrm{NMR}\left(150 \mathrm{MHz}, \mathrm{CDCl}_{3}\right) \delta 170.0,169.5,138.8,138.4$, $128.3,128.2,127.6,127.5,127.4,127.2$, 99.0, 83.6, 83.4, 82.8, 82.0, 79.8, 79.6, 78.9, 78.4, 77.5, 77.4, 77.20, 77.16, 77.00, 76.97, 76.8, 76.7, 76.31, 76.27, 76.0, 75.8, 75.7, 75.4, 75.2, 75.0, 73.0, 72.3, 72.0, 71.4, 70.8, 70.2, 70.1, 68.8, 67.3, 48.1, 44.6, 44.2, 38.3, 38.1, 37.45, 37.43, 36.3, 35.2 (x2), 35.1, 33.0, 32.9, 31.9, 29.33, 29.31, 25.04, 25.00, 21.2, 21.1, 17.8, 16.7, 16.6; HRFABMS $m / z$ calcd for $\mathrm{C}_{71} \mathrm{H}_{96} \mathrm{O}_{21} \mathrm{Na}\left(\mathrm{MNa}^{+}\right)$ 1307.6342, found 1307.6365 .

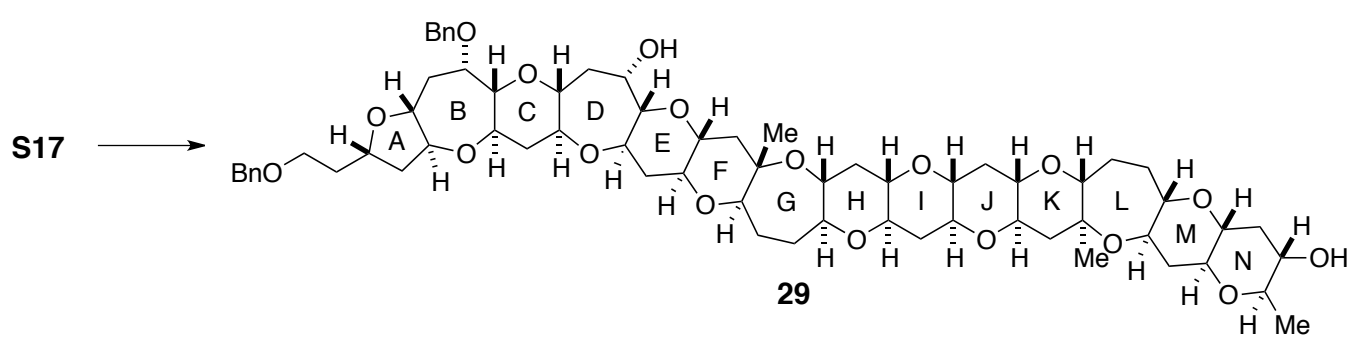

Reduction of S17. To a solution of methyl acetal S17 (6.3 $\mathrm{mg}, 0.0049 \mathrm{mmol})$ in $\mathrm{CH}_{2} \mathrm{Cl}_{2}(0.2 \mathrm{~mL})$ at $-20{ }^{\circ} \mathrm{C}$ were added $\mathrm{Et}_{3} \mathrm{SiH}(0.0157 \mathrm{~mL}, 0.098 \mathrm{mmol})$ and TMSOTf $(0.0035 \mathrm{~mL}, 0.0196 \mathrm{mmol})$, and the reaction mixture was stirred at $-20{ }^{\circ} \mathrm{C}$ for $16 \mathrm{~h}$. The reaction was quenched with saturated aqueous $\mathrm{NaHCO}_{3}$ solution, and the reaction mixture was extracted with EtOAc. The extract was washed with brine, dried, and concentrated under reduced pressure to afford crude tetradecacyclic acetate $(7.0 \mathrm{mg})$.

A mixture of above crude tetradecacyclic acetate and $\mathrm{NaOH}(0.2 \mathrm{~mL}, 2.5 \mathrm{M}$ solution in $\mathrm{MeOH}, 0.5 \mathrm{mmol})$ in THF $(0.3 \mathrm{~mL})$ was stirred at room temperature for 2.5 $h$. The reaction was quenched by saturated aqueous $\mathrm{NH}_{4} \mathrm{Cl}$ solution and the reaction mixture was extracted with EtOAc. The extract was washed with brine, dried, and concentrated under reduced pressure. Purification by flash chromatography $(100 \%$ 
EtOAc) afforded tetradecacyclic ether $29(4.5 \mathrm{mg}, 78 \%)$ as a colorless solid.

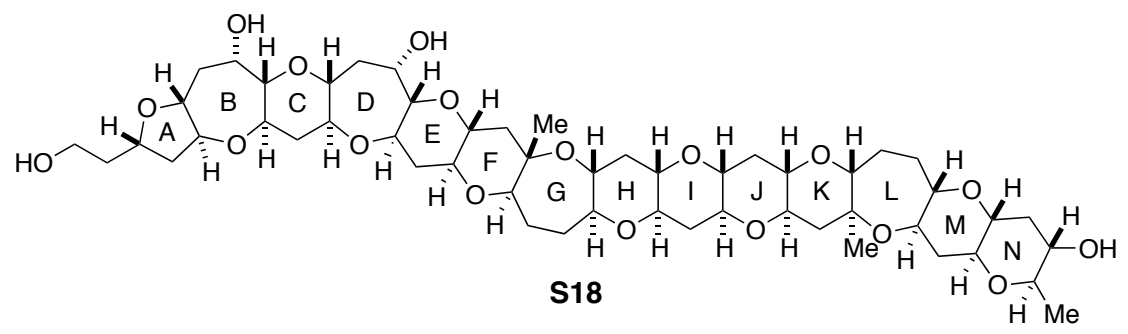

Tetraol S18. A mixture of dibenzyl ether $29(27.9 \mathrm{mg}, 0.0238 \mathrm{mmol})$ and $20 \% \mathrm{w} / \mathrm{w}$ $\mathrm{Pd}(\mathrm{OH})_{2}-\mathrm{C}(2.8 \mathrm{mg})$ in THF $(1 \mathrm{~mL})$ was stirred under a hydrogen atmosphere at room temperature for $24 \mathrm{~h}$ and $50{ }^{\circ} \mathrm{C}$ for $7 \mathrm{~h}$. The resulting gray suspension was filtered through a Celite pad and the pad was washed with $\mathrm{CH}_{2} \mathrm{Cl}_{2}$ and pyridine. The filtrate and washings were combined and concentrated under reduced pressure. Purification by flash chromatography $\left(10 \% \mathrm{MeOH}\right.$ in $\left.\mathrm{CH}_{2} \mathrm{Cl}_{2}\right)$ afforded tetraol $\mathbf{S 1 8}(19.6 \mathrm{mg}, 83 \%)$ as a colorless solid. $\mathrm{Mp}>320{ }^{\circ} \mathrm{C}$; $[\alpha]^{22}{ }_{\mathrm{D}}+31.5$ (c 0.17, $\left.\mathrm{CHCl}_{3}\right)$; IR $\left(\mathrm{CHCl}_{3}\right)$ 3427, 2937, 2873, 1457, $1067 \mathrm{~cm}^{-1}$; ${ }^{1} \mathrm{H}$ NMR (600 MHz, pyridine-d $\left.)_{5}\right) 6.86(2 \mathrm{H}$, br s), $6.73(1 \mathrm{H}, \mathrm{br}$ s), $5.88(1 \mathrm{H}$, br s), $4.75(1 \mathrm{H}, \mathrm{ddd}, J=9.3,7.8,6.6 \mathrm{~Hz}), 4.57(1 \mathrm{H}, \mathrm{d}, J=6.1 \mathrm{~Hz}), 4.45$ $(1 \mathrm{H}, \mathrm{m}), 4.41(1 \mathrm{H}, \mathrm{ddd}, J=11.7,9.2,4.8 \mathrm{~Hz}), 4.41-4.34(2 \mathrm{H}, \mathrm{m}), 4.24(1 \mathrm{H}, \mathrm{ddd}, J=$ 11.3, 9.2, $4.5 \mathrm{~Hz}), 3.99(1 \mathrm{H}, \mathrm{dq}, J=10.6,6.8 \mathrm{~Hz}), 3.97(1 \mathrm{H}, \mathrm{dq}, J=10.6,6.8 \mathrm{~Hz}), 3.79$ $(1 \mathrm{H}, \mathrm{q}, J=8.3 \mathrm{~Hz}), 3.73(1 \mathrm{H}, \mathrm{ddd}, J=11.2,9.3,5.0 \mathrm{~Hz}), 3.72(1 \mathrm{H}, \mathrm{ddd}, J=11.2,9.3$, $4.8 \mathrm{~Hz}), 3.61(1 \mathrm{H}, \mathrm{m}), 3.57(1 \mathrm{H}, \mathrm{td}, J=9.3,3.3 \mathrm{~Hz}), 3.50(1 \mathrm{H}, \mathrm{dq}, J=8.8,6.0 \mathrm{~Hz})$, 3.40-3.34 (2H, m), 3.33-3.10 (14H, m), 2.70-2.62 (3H, m), 2.57 (1H, dt, $J=11.0,4.0$ $\mathrm{Hz}), 2.54-2.49$ (3H, m), $2.44(1 \mathrm{H}, \mathrm{dt}, J=11.9,4.4 \mathrm{~Hz}), 2.40(1 \mathrm{H}, \mathrm{dt}, J=12.1,4.4 \mathrm{~Hz})$, $2.34(1 \mathrm{H}, \mathrm{ddd}, J=15.6,4.6,3.3 \mathrm{~Hz}), 2.28(1 \mathrm{H}, \mathrm{dd}, J=11.5,4.2 \mathrm{~Hz}), 2.26(1 \mathrm{H}, \mathrm{dd}, J=$ 11.5, 4.0 Hz), 2.23-2.08 (4H, m), 2.07-2.01 (2H, m), 1.63-1.97 (16H, m), 1.50 (3H, d, $J=6.1 \mathrm{~Hz}), 1.36(3 \mathrm{H}, \mathrm{s}), 1.35(3 \mathrm{H}, \mathrm{s}) ;{ }^{13} \mathrm{C}$ NMR $\left(150 \mathrm{MHz}\right.$, pyridine-d $\left.\mathrm{d}_{5}\right) \delta 85.7,84.1$, 83.8, 83.6, 83.0, 80.6, 80.1, 79.9, 79.4, 79.3, 77.9, 77.7, 77.5, 77.4, 77.1 (x2), 76.63, 76.60, 76.57, 76.45, 76.39, 76.3, 76.2, 75.3, 73.4, 71.3, 71.0, 70.9, 70.6, 70.5, 70.2, 59.2, $45.4,45.3,40.4,39.9,39.8,38.9,38.5,38.2,37.9,37.4,36.8,35.92,35.88,29.9,29.8$, 25.6, 25.5, 18.6, 16.8 (x2); HRFABMS $m / z$ calcd for $\mathrm{C}_{52} \mathrm{H}_{78} \mathrm{O}_{18} \mathrm{Na}\left(\mathrm{MNa}^{+}\right)$1013.5086, found 1013.5109. The spectral data of $\mathbf{S 1 8}$ was identical to the reported data: Tsukano, C.; Ebine, M.; Sasaki, M. J. Am. Chem. Soc. 2005, 127, 4326-4335. 


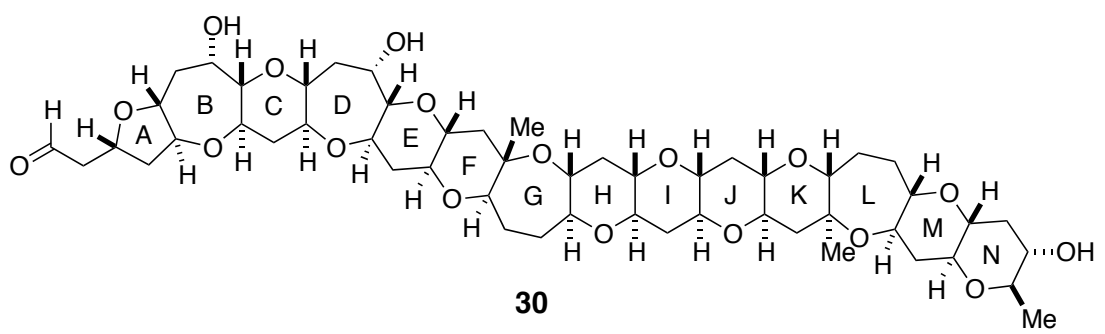

Aldehyde 30. To a suspension of tetraol $\mathbf{S 1 8}(15.6 \mathrm{mg}, 0.0157 \mathrm{mmol})$ in $\mathrm{CH}_{2} \mathrm{Cl}_{2}(2 \mathrm{~mL})$ was added DMN-AZADO $\left(0.28 \mathrm{~mL}, 3.8 \mathrm{mg} / \mathrm{mL}\right.$ solution in $\mathrm{CH}_{2} \mathrm{Cl}_{2}, 1.1 \mathrm{mg}, 0.0066$ $\mathrm{mmol})$ and $\mathrm{PhI}(\mathrm{OAc})_{2}(7.6 \mathrm{mg}, 0.024 \mathrm{mmol})$, and the reaction mixture was stirred at room temperature for $3 \mathrm{~h}$. The additional $\mathrm{PhI}(\mathrm{OAc})_{2}(6.9 \mathrm{mg}, 0.021 \mathrm{mmol})$ was added, and the reaction mixture was stirred for an additional $3 \mathrm{~h}$. At the end of the reaction, the reaction mixture was turned into a solution. The reaction mixture was directly purified by flash chromatography $\left(\mathrm{CH}_{2} \mathrm{Cl}_{2} \rightarrow 10 \% \mathrm{MeOH} / \mathrm{CH}_{2} \mathrm{Cl}_{2}\right)$. Aldehyde 30 (13.5 mg, 87\%) was obtained as a colorless solid. Mp 260-280 ${ }^{\circ} \mathrm{C}$ (decomposed); $[\alpha]^{26}{ }_{\mathrm{D}}+26.8$ (c 1.13, $\left.\mathrm{CHCl}_{3}\right)$; IR $\left(\mathrm{CHCl}_{3}\right)$ 3434, 2947, 2874, 1722, 1456, $1069 \mathrm{~cm}^{-1} ;{ }^{1} \mathrm{H}$ NMR $(600 \mathrm{MHz}$, $\left.\mathrm{CDCl}_{3}\right) \delta 9.79(1 \mathrm{H}, \mathrm{t}, J=1.7 \mathrm{~Hz}), 4.48(1 \mathrm{H}$, quintet, $J=6.8 \mathrm{~Hz}), 4.25(1 \mathrm{H}, \mathrm{d}, J=8.1$ $\mathrm{Hz}), 4.20(1 \mathrm{H}, \mathrm{m}), 4.10(1 \mathrm{H}, \mathrm{ddd}, J=9.2,7.7,7.0 \mathrm{~Hz}), 3.86(1 \mathrm{H}, \mathrm{ddd}, J=11.4,9.1,4.9$ $\mathrm{Hz}), 3.73(1 \mathrm{H}, \mathrm{ddd}, J=11.7,9.2,4.6 \mathrm{~Hz}), 3.67$ (1H, ddd, $J=11.6,9.6,3.9 \mathrm{~Hz})$, 3.61-3.52 (3H, m), 3.38-3.30 (2H, m), 3.29-3.24 (2H, m), 3.23-3.18 (3H, m), 3.15-2.95 (12H, m), $2.88(1 \mathrm{H}$, br s), $2.78(1 \mathrm{H}$, br s), $2.71(1 \mathrm{H}, \mathrm{ddd}, J=16.7,7.0,2.0$ Hz), 2.60 (1H, ddd, $J=16.7,5.5,1.5 \mathrm{~Hz}), 2.51$ (1H, dt, $J=14.7,7.6 \mathrm{~Hz}), 2.42-2.28$ (6H, m), 2.26-2.20 (2H, m), 2.17-1.98 (7H, m), 1.92-1.84 (2H, m), 1.80-1.65 (5H, m), $1.60(1 \mathrm{H}$, br s), 1.60-1.38 (9H, m), 1.31 (3H, d, $J=6.1 \mathrm{~Hz}), 1.29(3 \mathrm{H}, \mathrm{s}), 1.27(3 \mathrm{H}, \mathrm{s})$; ${ }^{13} \mathrm{C}$ NMR $\left(150 \mathrm{MHz}, \mathrm{CDCl}_{3}\right) \delta$ 200.6, 84.1, 83.61, 83.59, 82.7, 81.9, 80.0, 79.8, 79.6, 78.9, 78.1, 77.5, 77.1 (x2), 77.0 (x2), 76.8 (x2), 76.5, 76.1, 76.0, 75.71 (x2), 75.66, 73.7, $72.1,71.5,70.5,70.3,70.2,70.1,69.9,49.8,44.6,44.5,38.6,38.3,37.9,37.5,37.4$, 36.5, 36.0, 35.2, 35.1, 34.4, 29.40, 29.38, 25.1 (x2), 17.8, 16.60, 16.58; HRFABMS m/z calcd for $\mathrm{C}_{52} \mathrm{H}_{76} \mathrm{O}_{18} \mathrm{Na}\left(\mathrm{MNa}^{+}\right)$1011.4929, found 1011.4946. 


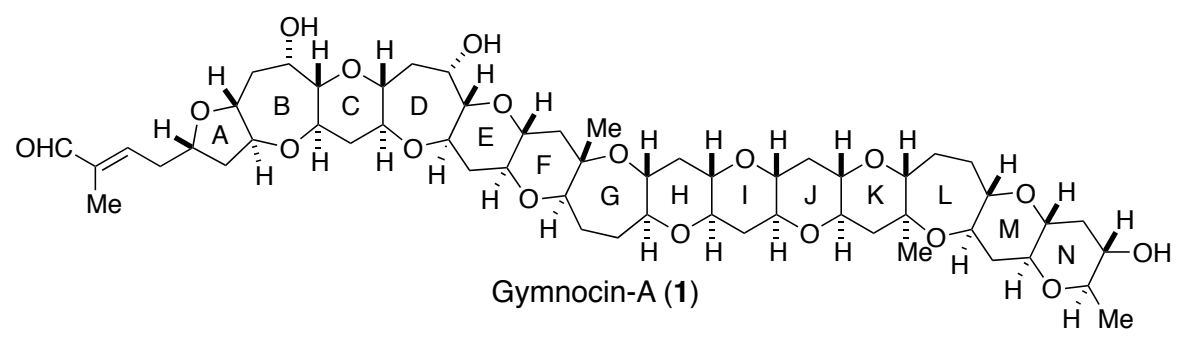

Gymnocin-A (1). A solution of aldehyde 30 (13.5 $\mathrm{mg}, 0.0136 \mathrm{mmol})$ and 2-(triphenylphosphoranylidene)propionaldehyde (31) $(70 \mathrm{mg}, 0.220 \mathrm{mmol})$ in $\mathrm{C}_{6} \mathrm{H}_{5} \mathrm{Cl}$ $(1.4 \mathrm{~mL})$ was stirred at $100{ }^{\circ} \mathrm{C}$ for $7 \mathrm{~h}$. The reaction mixture was allowed to cool to room temperature, and then directly purified by flash chromatography $\left(\mathrm{CH}_{2} \mathrm{Cl}_{2} \rightarrow 10 \%\right.$ $\left.i-\mathrm{PrOH} / \mathrm{CH}_{2} \mathrm{Cl}_{2}\right)$ to afford gymnocin-A (1) $(6.7 \mathrm{mg}, 48 \%$, trans:cis $=91: 9)$ as a colorless solid. $\mathrm{Mp}>320{ }^{\circ} \mathrm{C} ;[\alpha]^{26}{ }_{\mathrm{D}}+22.9\left(c 0.56, \mathrm{CHCl}_{3}\right)$; IR $\left(\mathrm{CHCl}_{3}\right) 3419,2933,2873,1683$, $1067 \mathrm{~cm}^{-1} ;{ }^{1} \mathrm{H} \mathrm{NMR}\left(600 \mathrm{MHz}, \mathrm{CDCl}_{3}\right) \delta 9.42(1 \mathrm{H}, \mathrm{s}), 6.51(1 \mathrm{H}, \mathrm{tq}, J=7.0,1.3 \mathrm{~Hz})$, $4.23(1 \mathrm{H}, \mathrm{d}, J=7.7 \mathrm{~Hz}), 4.21-4.14(2 \mathrm{H}, \mathrm{m}), 4.06(1 \mathrm{H}, \mathrm{ddd}, J=9.0,8.0,7.0 \mathrm{~Hz}), 3.83$ $(1 \mathrm{H}, \mathrm{ddd}, J=11.3,8.9,5.0 \mathrm{~Hz}), 3.71(1 \mathrm{H}, \mathrm{ddd}, J=11.2,9.3,4.6 \mathrm{~Hz}), 3.64(1 \mathrm{H}, \mathrm{ddd}, J$ $=11.2,9.5,4.2 \mathrm{~Hz}), 3.57(1 \mathrm{H}, \mathrm{dt}, J=10.1,7.5 \mathrm{~Hz}), 3.56-3.49(2 \mathrm{H}, \mathrm{m}), 3.32(1 \mathrm{H}, \mathrm{ddd}, J$ $=10.6,9.5,5.0 \mathrm{~Hz}), 3.29(1 \mathrm{H}, \mathrm{ddd}, J=9.1,8.1,4.2 \mathrm{~Hz}), 3.26-3.21(2 \mathrm{H}, \mathrm{m}), 3.21-3.14$ $(3 \mathrm{H}, \mathrm{m}), 3.11(1 \mathrm{H}, \mathrm{d}, J=9.0 \mathrm{~Hz}), 3.10-2.92(11 \mathrm{H}, \mathrm{m}), 2.82(1 \mathrm{H}$, br s $), 2.70(1 \mathrm{H}, \mathrm{br} \mathrm{s})$, 2.60-2.53 (2H, m), 2.49 (1H, ddd, $J=15.0,7.9,7.0 \mathrm{~Hz}), 2.40-2.25(6 \mathrm{H}, \mathrm{m}), 2.21(1 \mathrm{H}$, $\mathrm{dt}, J=12.4,4.6 \mathrm{~Hz}), 2.19(1 \mathrm{H}, \mathrm{dt}, J=12.4,4.6 \mathrm{~Hz}), 2.15-2.07(2 \mathrm{H}, \mathrm{m}), 2.06(1 \mathrm{H}, \mathrm{dd}, J$ $=12.3,4.2 \mathrm{~Hz}), 2.02(1 \mathrm{H}, \mathrm{dd}, J=12.3,4.2 \mathrm{~Hz}), 2.02-1.94(3 \mathrm{H}, \mathrm{m}), 1.89-1.81(2 \mathrm{H}, \mathrm{m})$, 1.80-1.65 (5H, m), $1.75(3 \mathrm{H}, \mathrm{d}, J=1.3 \mathrm{~Hz}), 1.55(1 \mathrm{H}, \mathrm{br} \mathrm{s}), 1.53(1 \mathrm{H}, \mathrm{t}, J=12.1 \mathrm{~Hz})$, $1.50(1 \mathrm{H}, \mathrm{t}, J=12.1 \mathrm{~Hz}), 1.49-1.38(6 \mathrm{H}, \mathrm{m}), 1.38(1 \mathrm{H}, \mathrm{q}, J=11.2 \mathrm{~Hz}), 1.28(3 \mathrm{H}, \mathrm{d}, J=$ $6.1 \mathrm{~Hz}), 1.26(3 \mathrm{H}, \mathrm{s}), 1.24(3 \mathrm{H}, \mathrm{s}) ;{ }^{13} \mathrm{C} \mathrm{NMR}\left(150 \mathrm{MHz}, \mathrm{CDCl}_{3}\right) \delta$ 195.1, 149.5, 141.0, 83.9, 83.63, 83.56, 82.7, 81.9, 80.1, 79.8, 79.6, 78.9, 78.1, 77.5, 77.1 (x2), 77.0 (x2), 76.8, 76.7, 76.5, 76.1, 76.0, 75.8, 75.71 (x2), 75.66, 73.5, 71.5, 70.5, 70.2 (x2), 70.1, 69.9, 44.60, 44.56, 38.6, 38.3, 37.7, 37.5, 37.4, 36.5, 36.1, 35.5, 35.2 (x2), 34.5, 29.42, 29.38, 25.1 (x2), 17.8, 16.6 (x2), 9.5; HRFABMS $m / z$ calcd for $\mathrm{C}_{55} \mathrm{H}_{80} \mathrm{O}_{18} \mathrm{Na}\left(\mathrm{MNa}^{+}\right)$ 1051.5242, found 1051.5269 . 


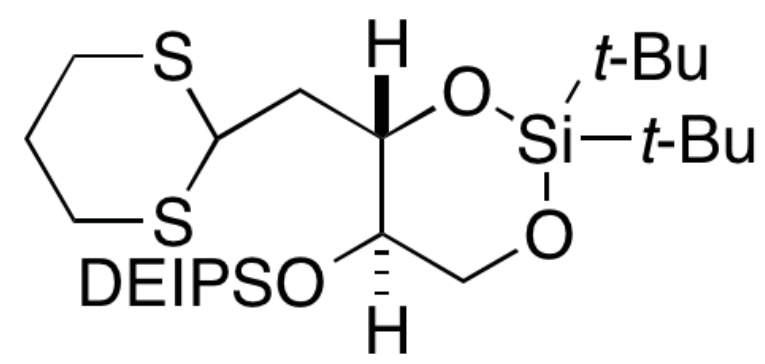

S2

${ }^{1} \mathrm{H}$ NMR $\left(\mathrm{CDCl}_{3}, 500 \mathrm{MHz}\right)$

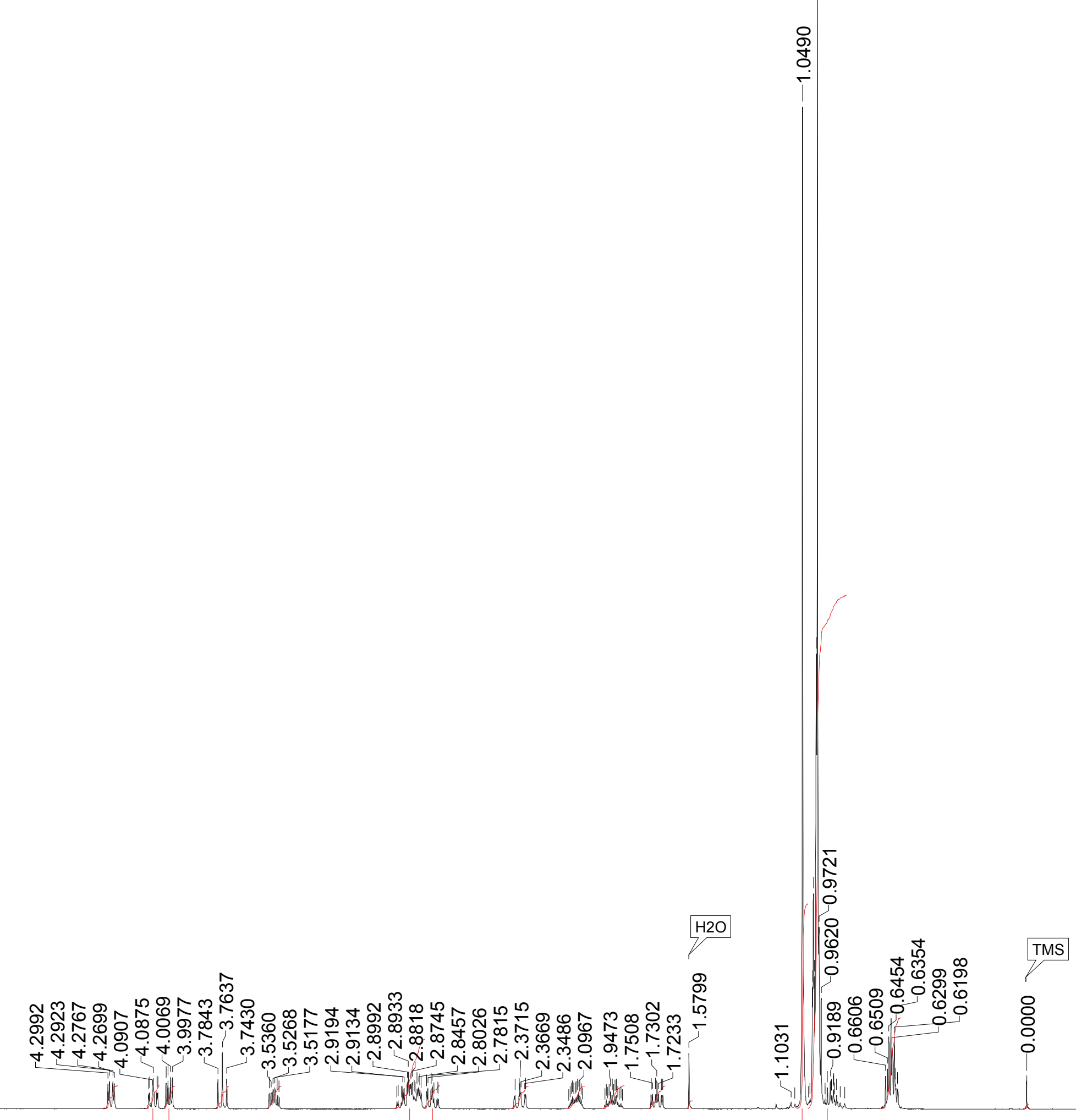

0.27

$\begin{array}{llllllll}1.011 .021 .00 & 1.00 & 1.00 & 3.061 .02 & 1.00 & 1.01 & 1.02 & 1.01 \\ 0.36\end{array}$

$9.0422 .67 \quad 4.02$ 0.18 
This report was created by ACD/NMR Processor Academic Edition. For more information go to www.acdlabs.com/nmrproc/

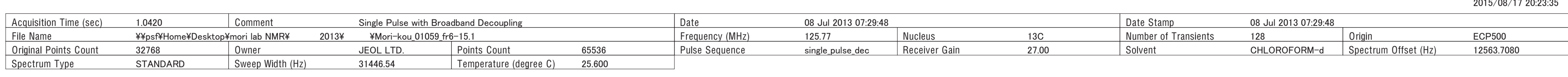

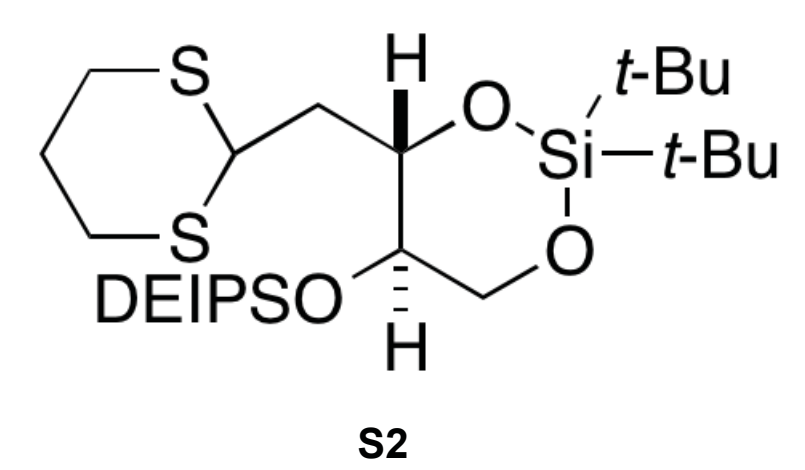

${ }^{13} \mathrm{C} \mathrm{NMR}\left(\mathrm{CDCl}_{3}, 125 \mathrm{MHz}\right)$
$\mathrm{CDCl} 3$

$$
\begin{aligned}
& \text { 융 }
\end{aligned}
$$

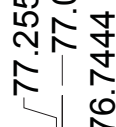
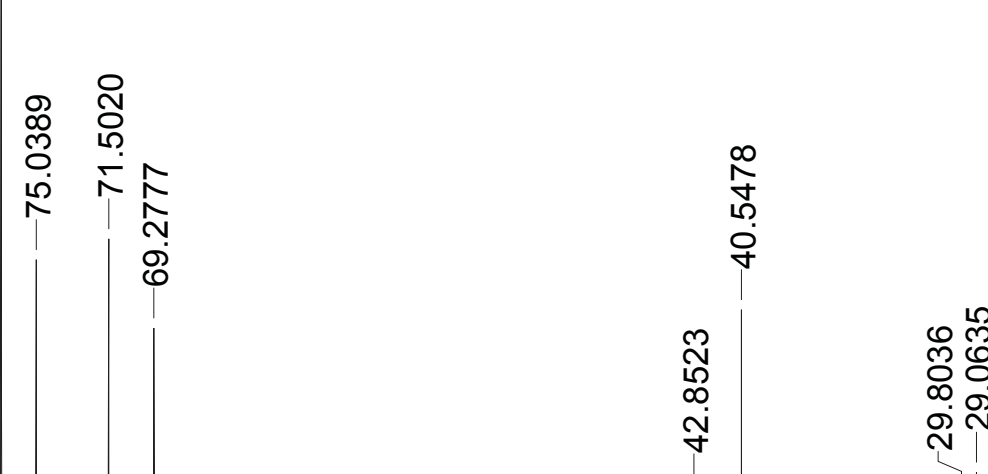
This report was created by ACD/NMR Processor Academic Edition. For more information go to www.acdlabs.com/nmrproc/
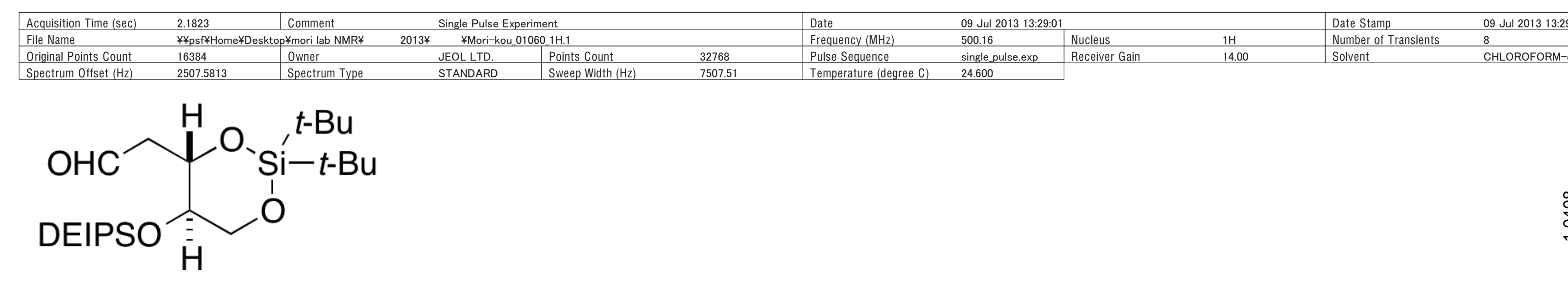

S3

${ }^{1} \mathrm{H} \mathrm{NMR}\left(\mathrm{CDCl}_{3}, 500 \mathrm{MHz}\right)$

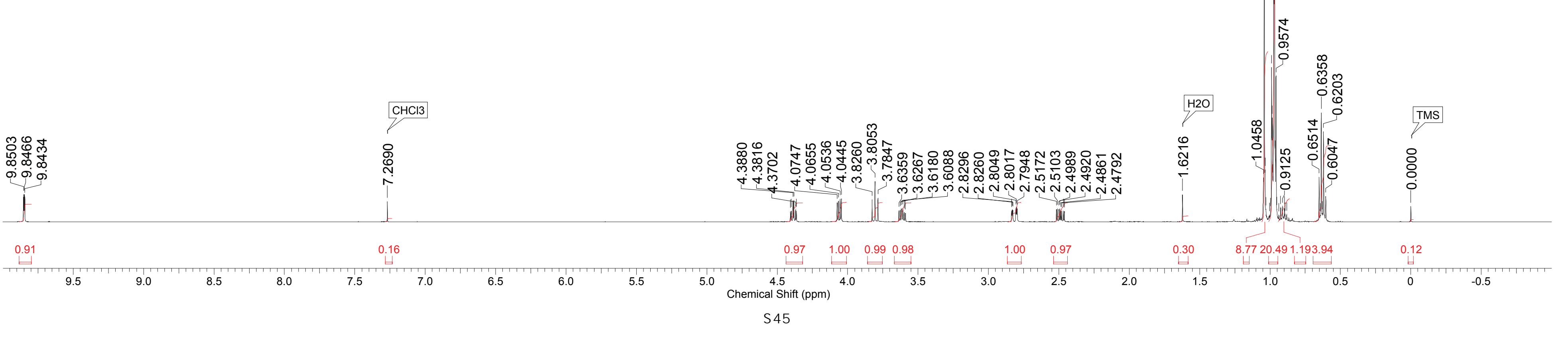


This report was created by ACD/NMR Processor Academic Edition. For more information go to www.acdlabs.com/nmrproc/



Spectrum Type JEOLLTD. Temperature (degree C) $\quad 2655360$ Pulse Sequence Receiver Gain

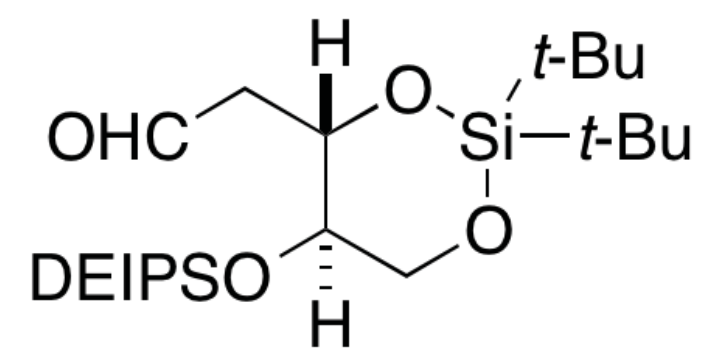

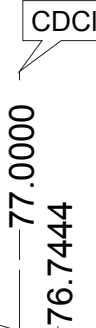

${ }^{13} \mathrm{C}$ NMR $\left(\mathrm{CDCl}_{3}, 125 \mathrm{MHz}\right)$
స్త్ర

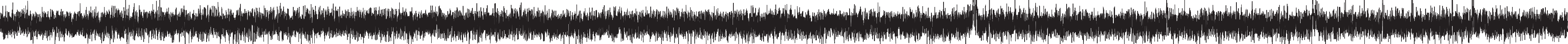


This report was created by ACD/NMR Processor Academic Edition. For more information go to www.acdlabs.com/nmrproc/
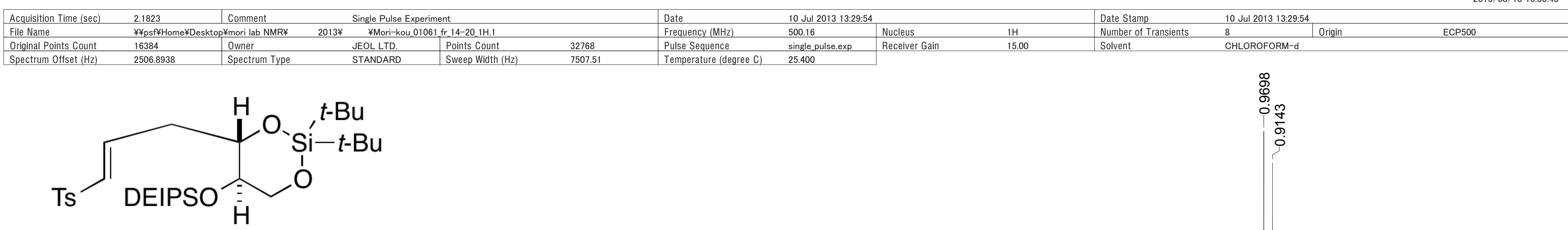

S4

${ }^{1} \mathrm{H} \mathrm{NMR}\left(\mathrm{CDCl}_{3}, 500 \mathrm{MHz}\right)$

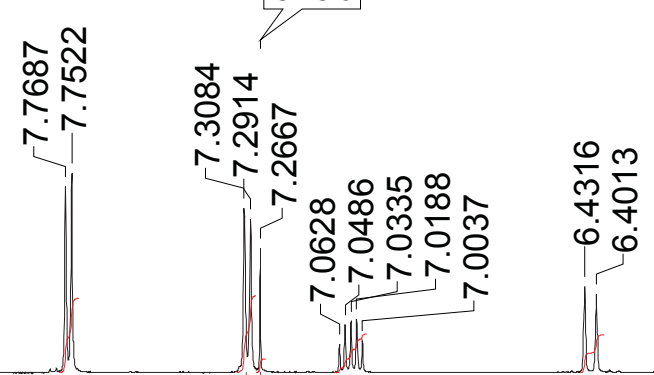

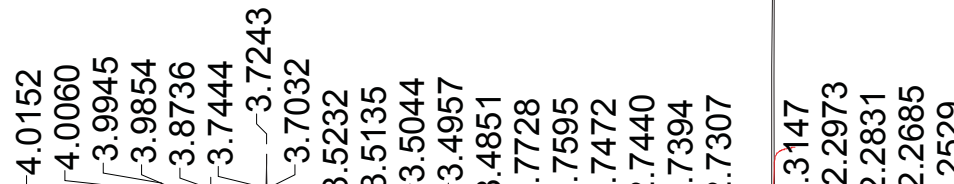

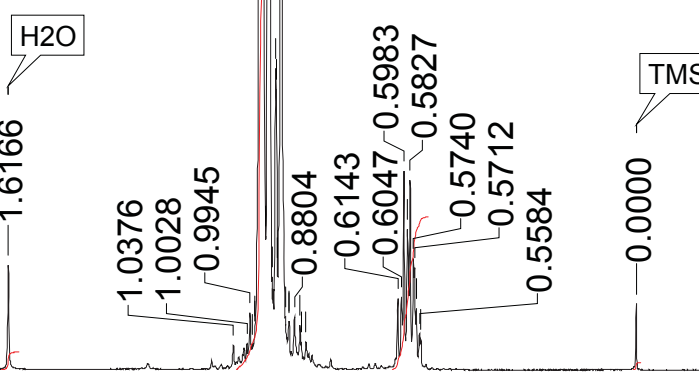

$1.92 \quad 1.970 .350 .99$

6.5

6.0

$1.001 .021 .04 \quad 1.01$

$0.99 \quad 3.071 .05$

0.46

$31.80 \quad 3.94$

0.18 
This report was created by ACD/NMR Processor Academic Edition. For more information go to www.acdlabs.com/nmrproc/
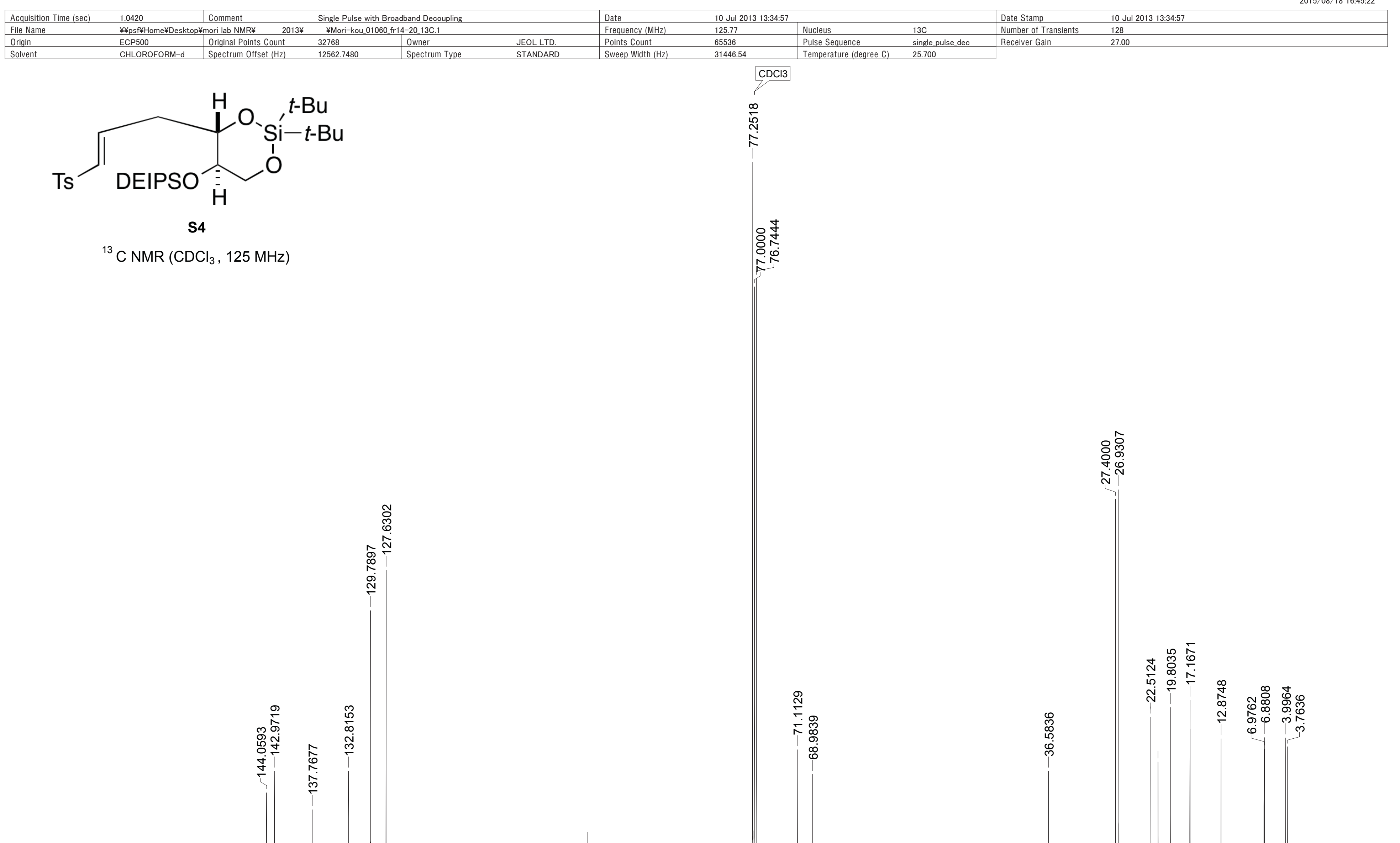

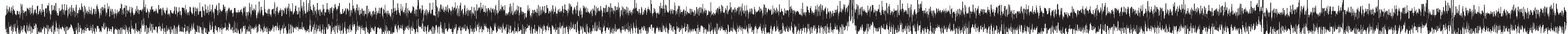

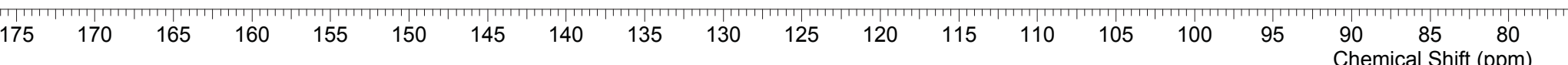




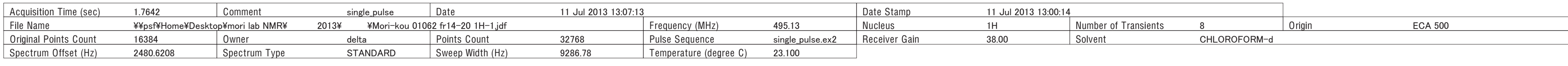

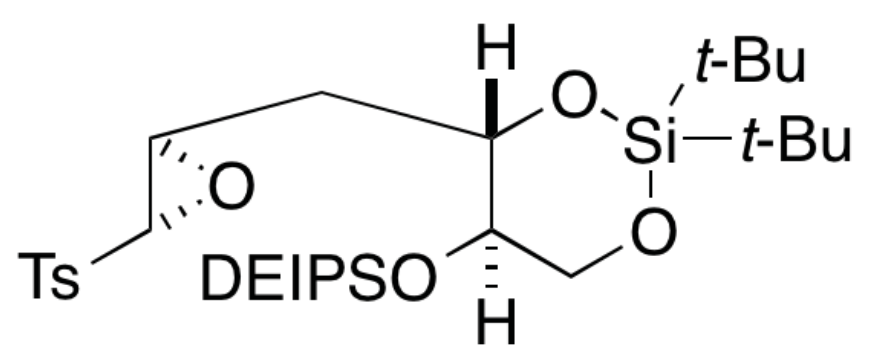

$8(\mathrm{dr}=86: 14)$

${ }^{1} \mathrm{H}$ NMR $\left(\mathrm{CDCl}_{3}, 500 \mathrm{MHz}\right)$

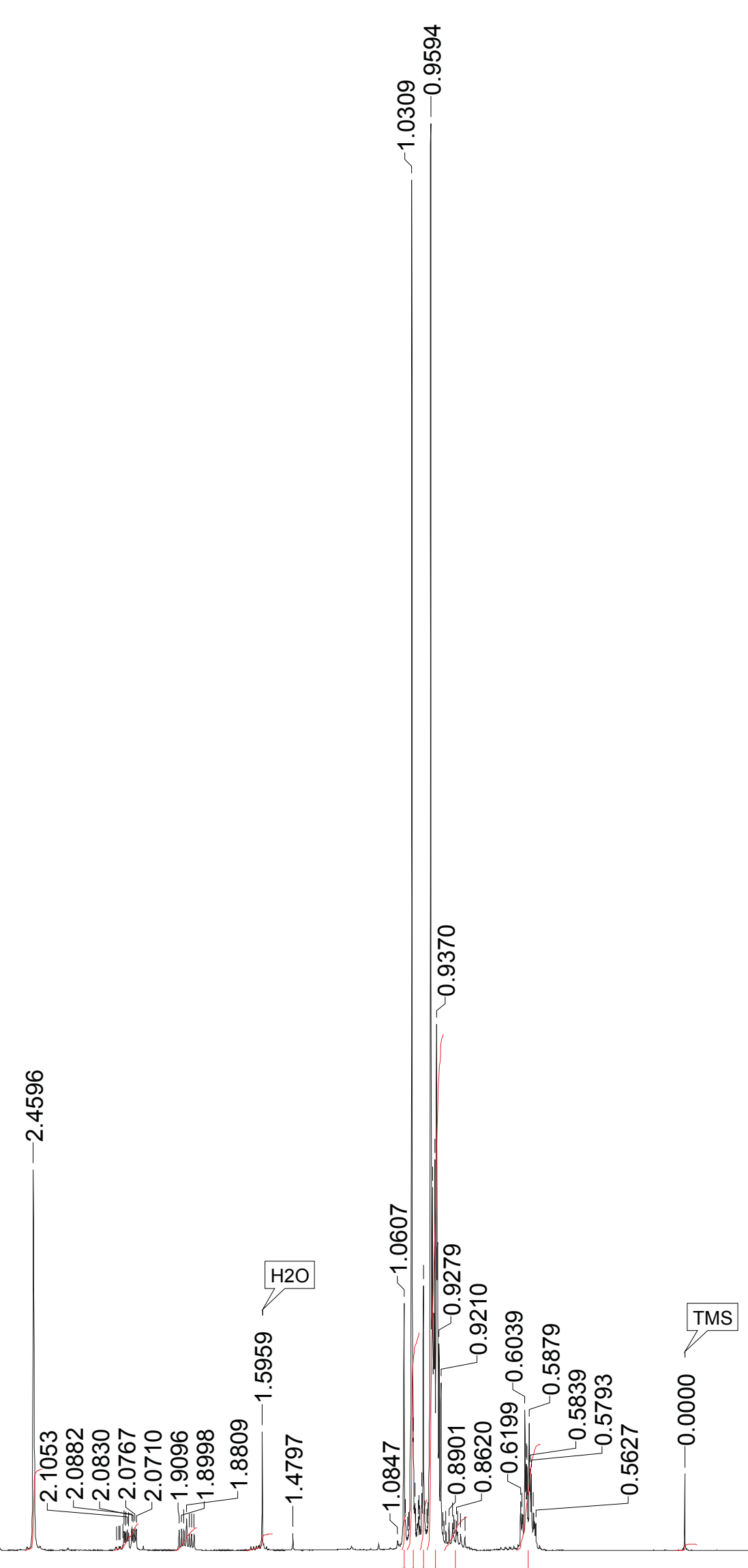

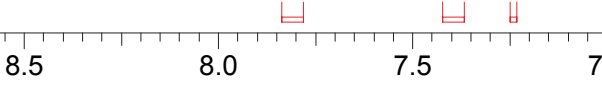

0.830 .990 .350 .851 .981 .00

3.00

$\begin{array}{llll}0.97 & 0.82 & 0.60 & 1.358 .031 .88 \\ 19.011 .233 .91\end{array}$ 0.21 
This report was created by ACD/NMR Processor Academic Edition. For more information go to www.acdlabs.com/nmrproc/



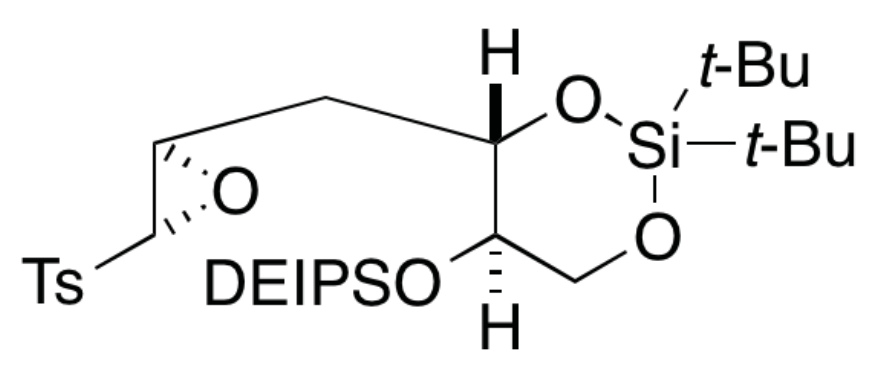

$8(\mathrm{dr}=86: 14)$

${ }^{13} \mathrm{C} \mathrm{NMR}\left(\mathrm{CDCl}_{3}, 125 \mathrm{MHz}\right)$

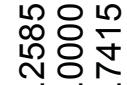 \\ 章它}

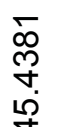

ลัก

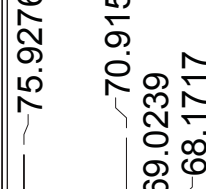

0
$\mathbb{N}$
0
10
10

0
$\stackrel{1}{L}$
$\infty$
$m$
$m$

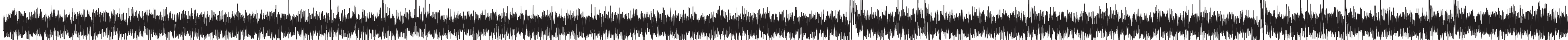


This report was created by ACD/NMR Processor Academic Edition. For more information go to www.acdlabs.com/nmrproc/

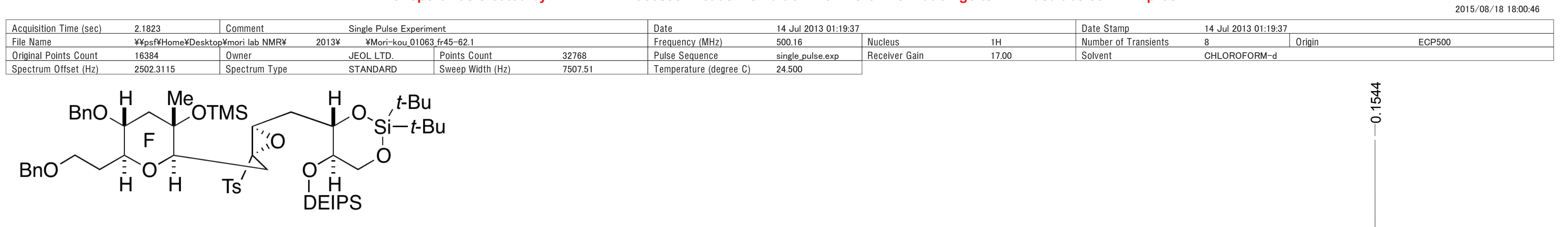

$10(\mathrm{dr}=91: 9)$

${ }^{1} \mathrm{H} \mathrm{NMR}\left(\mathrm{CDCl}_{3}, 500 \mathrm{MHz}\right)$

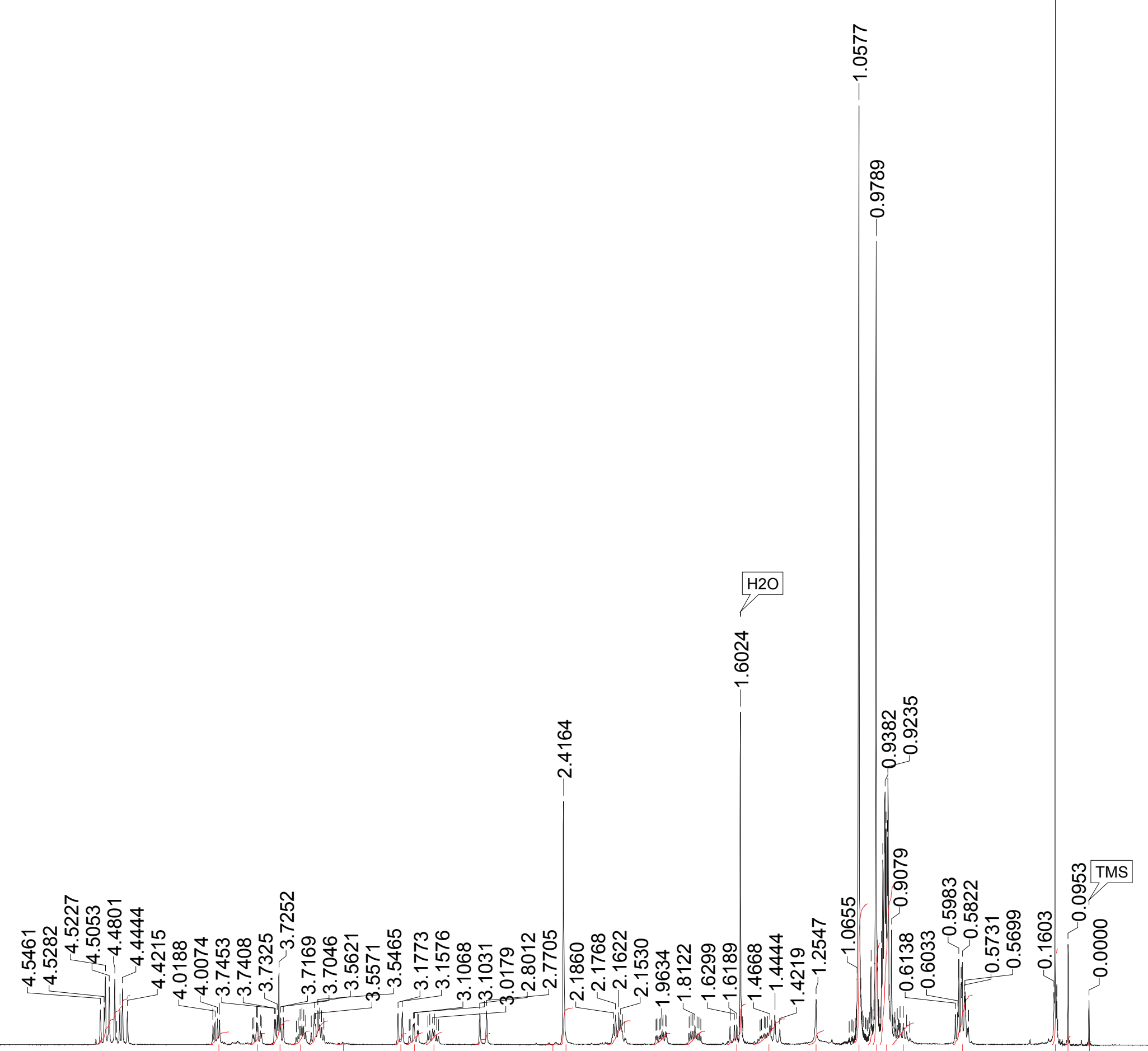

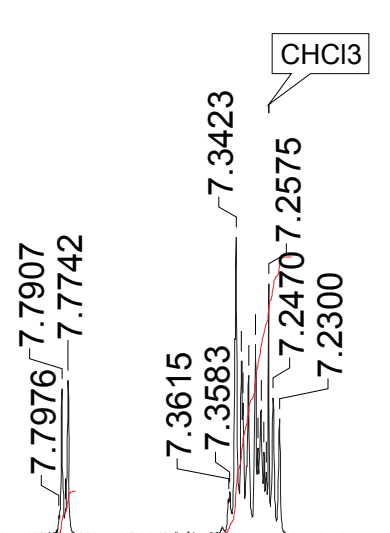

$1.94 \quad 12.37$

$3.93 \quad 1.000 .911 .921 .101 .890 .120 .890 .940 .970 .89$

$0.092 .93 \quad 1.91 \quad 0.921 .073 .232 .071 .1211 .210 .998 .0912 .581 .743 .947 .620 .630 .25$ 
This report was created by ACD/NMR Processor Academic Edition. For more information go to www.acdlabs.com/nmrproc/

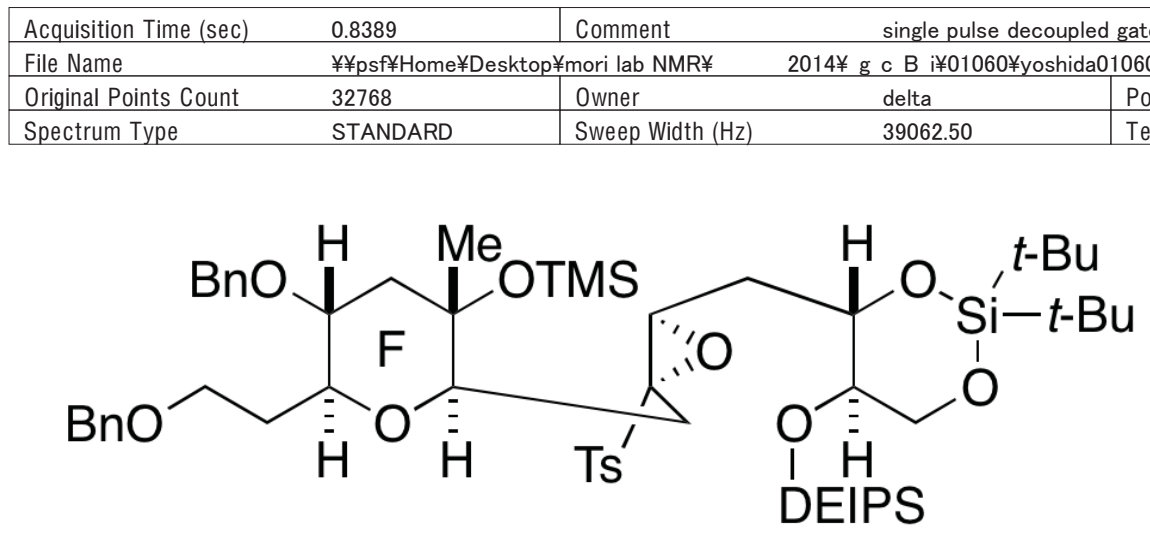

$10(d r=91: 9)$

${ }^{13} \mathrm{C}$ NMR $\left(\mathrm{CDCl}_{3}, 125 \mathrm{MHz}\right)$
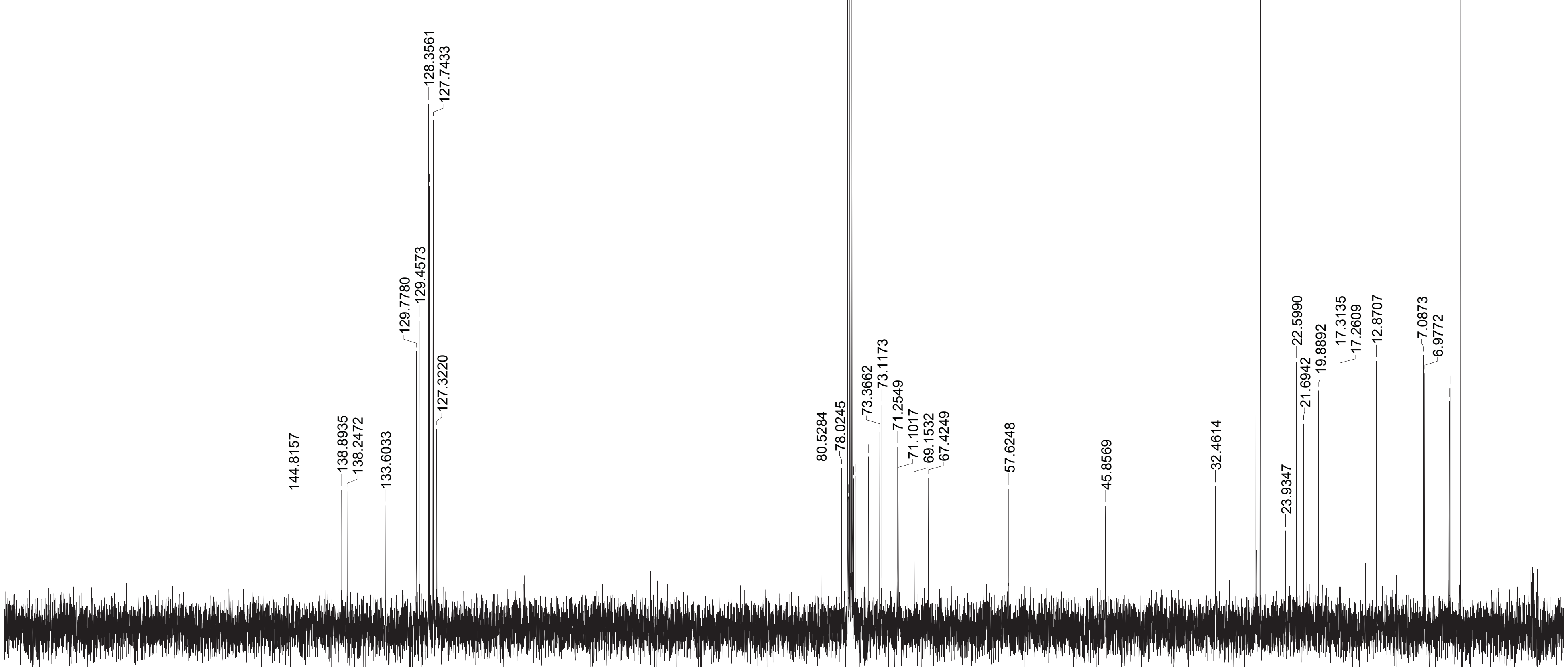

$\mathrm{CDCl3}$

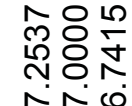

$$
\begin{aligned}
& \text { 송ำ }
\end{aligned}
$$

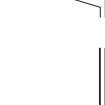



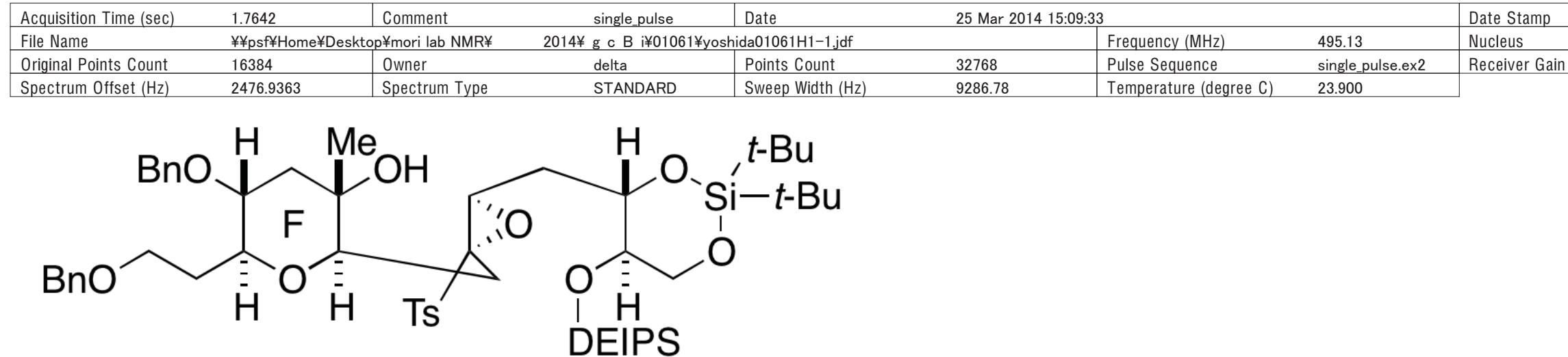

25 Mar 2014 15:00:52 Number of Transients
Solvent

S5 $(d r=91: 9)$

${ }^{1} \mathrm{H} \mathrm{NMR}\left(\mathrm{CDCl}_{3}, 500 \mathrm{MHz}\right)$

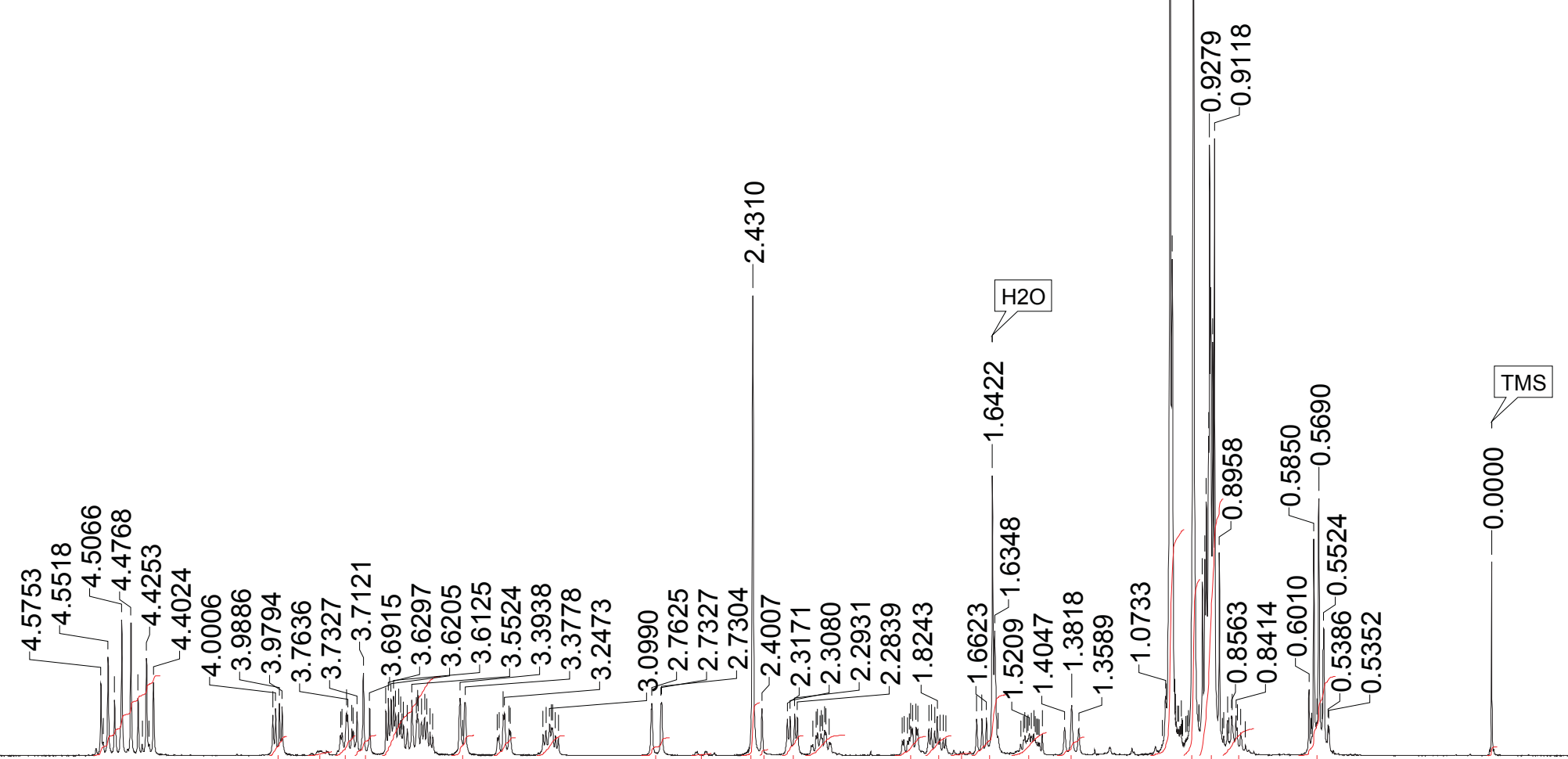

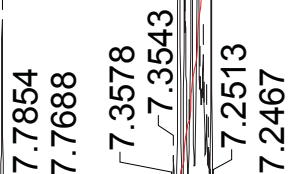

$\begin{array}{lll}4.10 & 0.97 & 0.191 .031 .03 \\ 4.041 .03 & 0.931 .010 .910 .092 .710 .301 .031 .040 .991 .020 .143 .131 .170 .9411 .589 .0513 .171 .374 .05\end{array}$ 
This report was created by ACD/NMR Processor Academic Edition. For more information go to www.acdlabs.com/nmrproc/

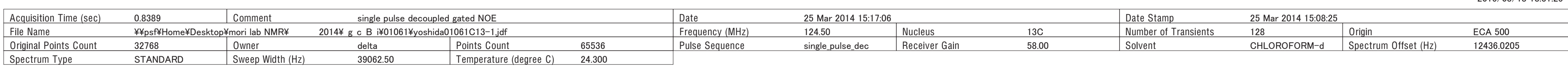

Spectrum Type

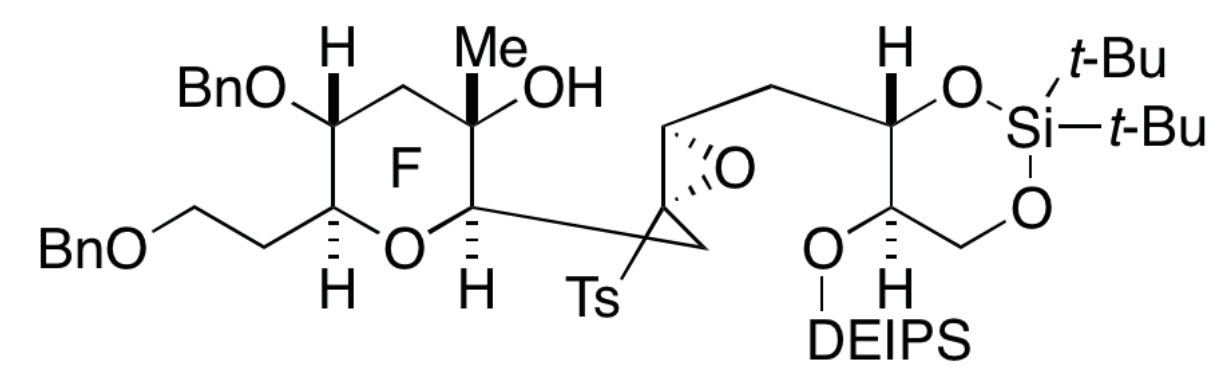

$\mathrm{CDCl3}$

S5 $(\mathrm{dr}=91: 9)$

${ }^{13} \mathrm{C} \mathrm{NMR}\left(\mathrm{CDCl}_{3}, 125 \mathrm{MHz}\right)$

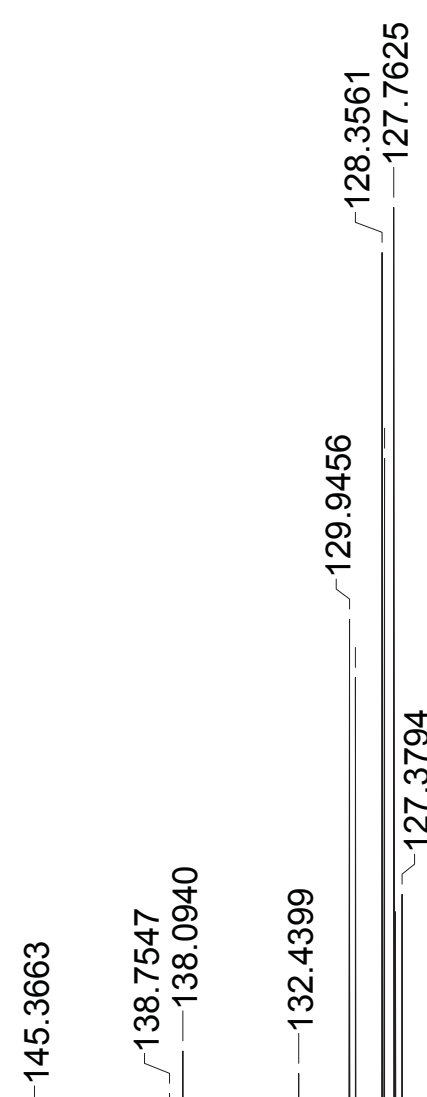

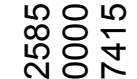 \\ Nise}
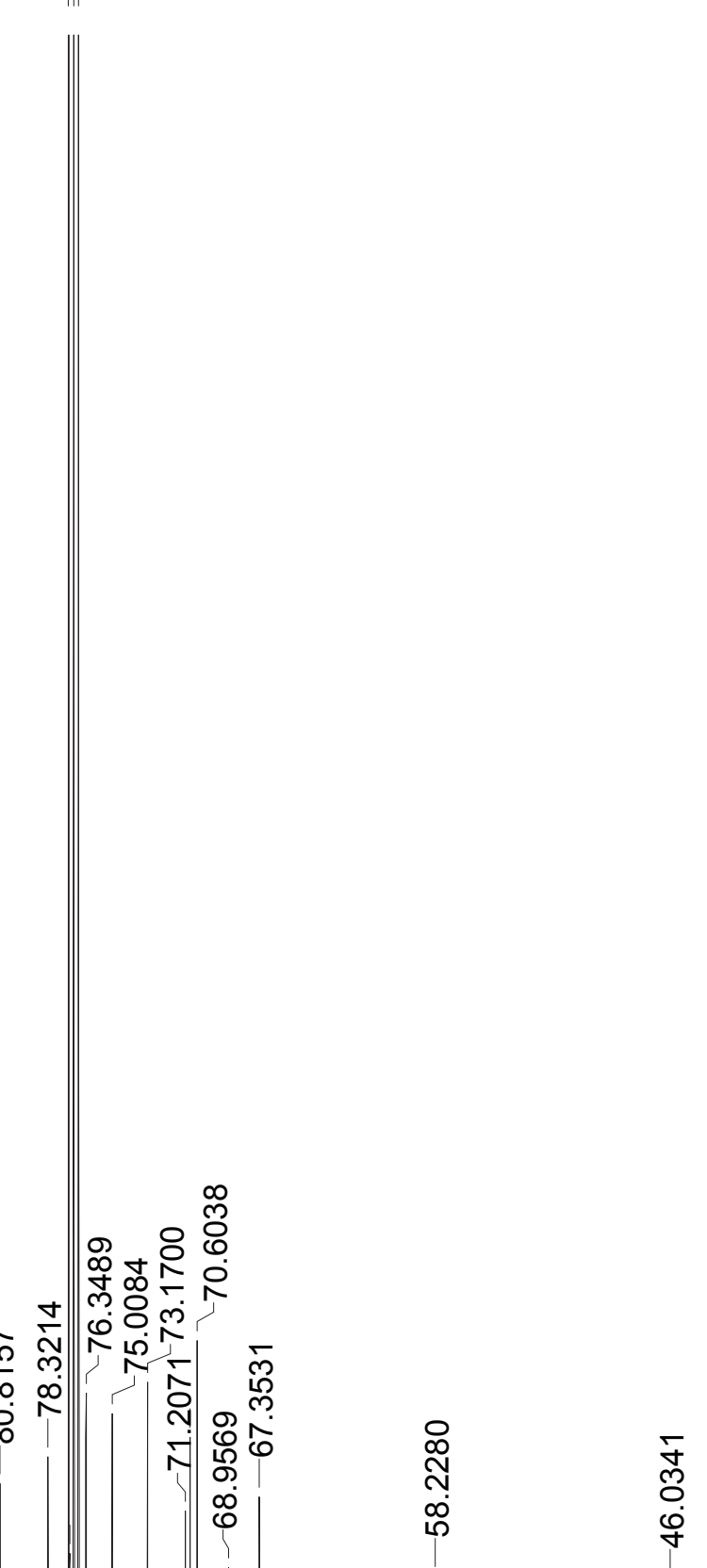

亦 กู่

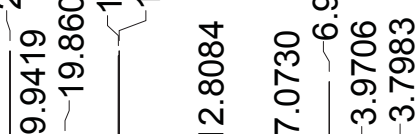



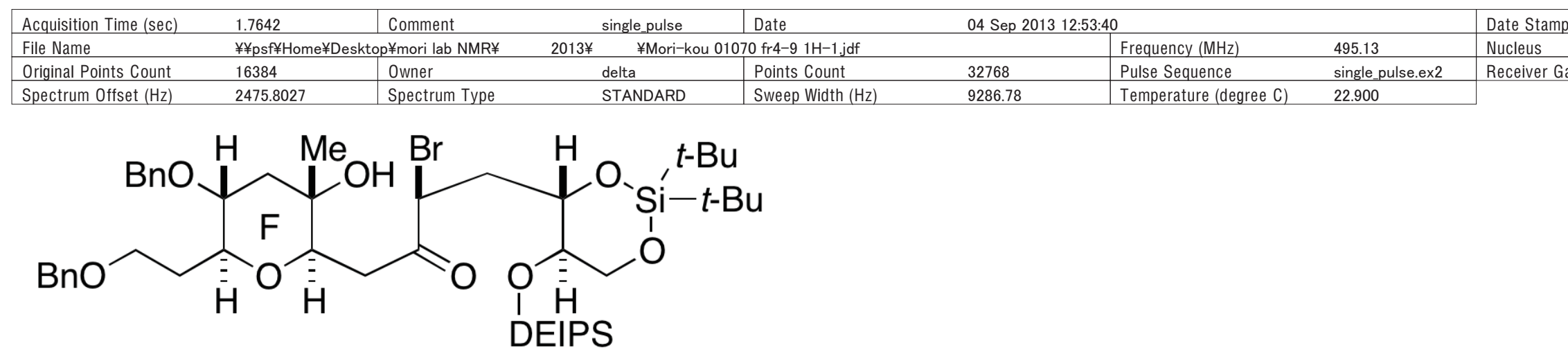

$04 \operatorname{Sep} 2013$ 12:51:52

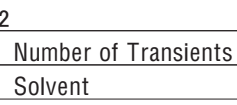

$11(\mathrm{dr}=91: 9)$

${ }^{1} \mathrm{H} \mathrm{NMR}\left(\mathrm{CDCl}_{3}, 500 \mathrm{MHz}\right)$

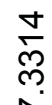
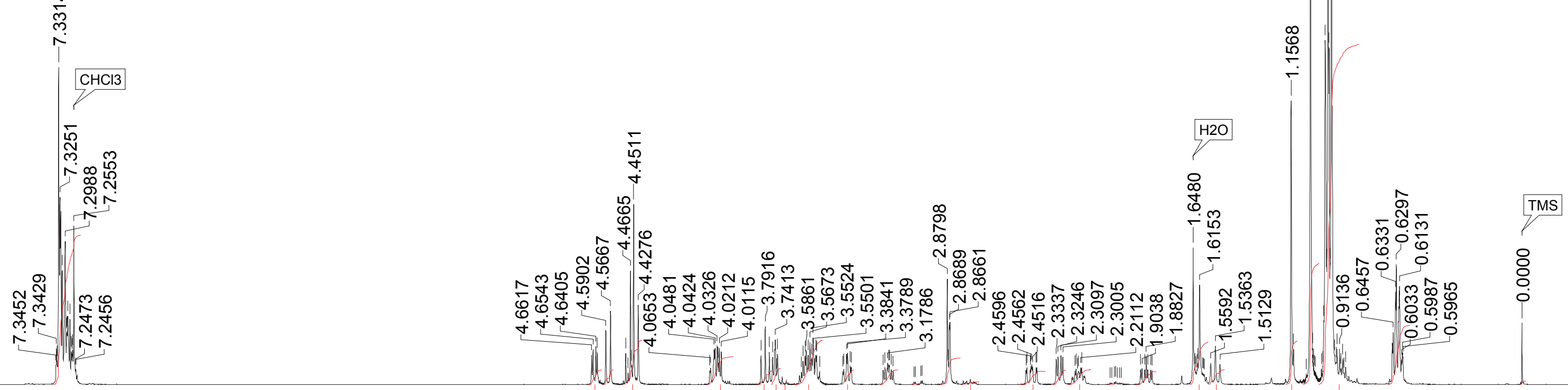

10.47

1.021 .023 .09

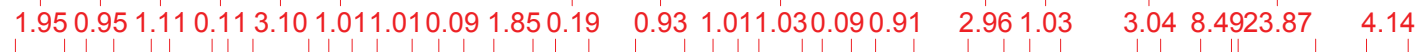


This report was created by ACD/NMR Processor Academic Edition. For more information go to www.acdlabs.com/nmrproc/

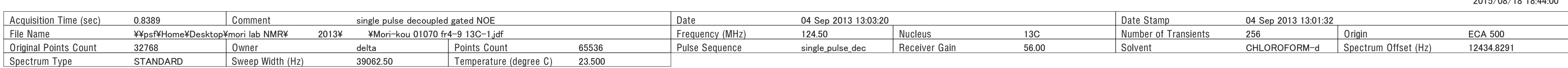

Spectrum Type

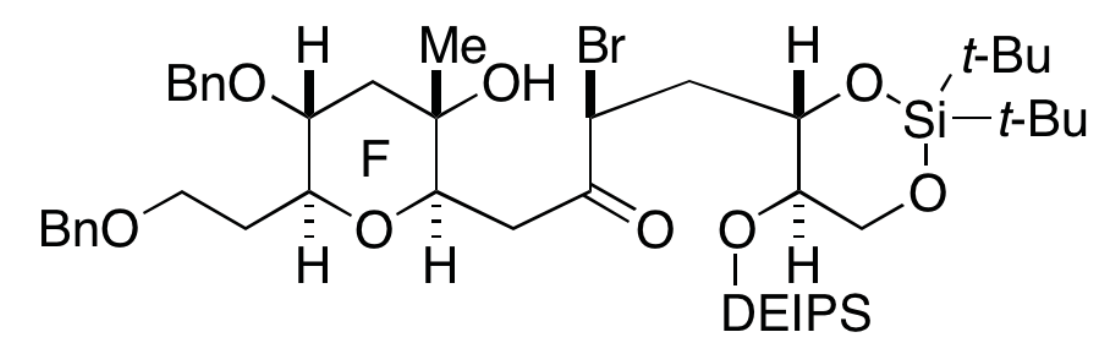

$11(\mathrm{dr}=91: 9)$

${ }^{13} \mathrm{C} \mathrm{NMR}\left(\mathrm{CDCl}_{3}, 125 \mathrm{MHz}\right)$

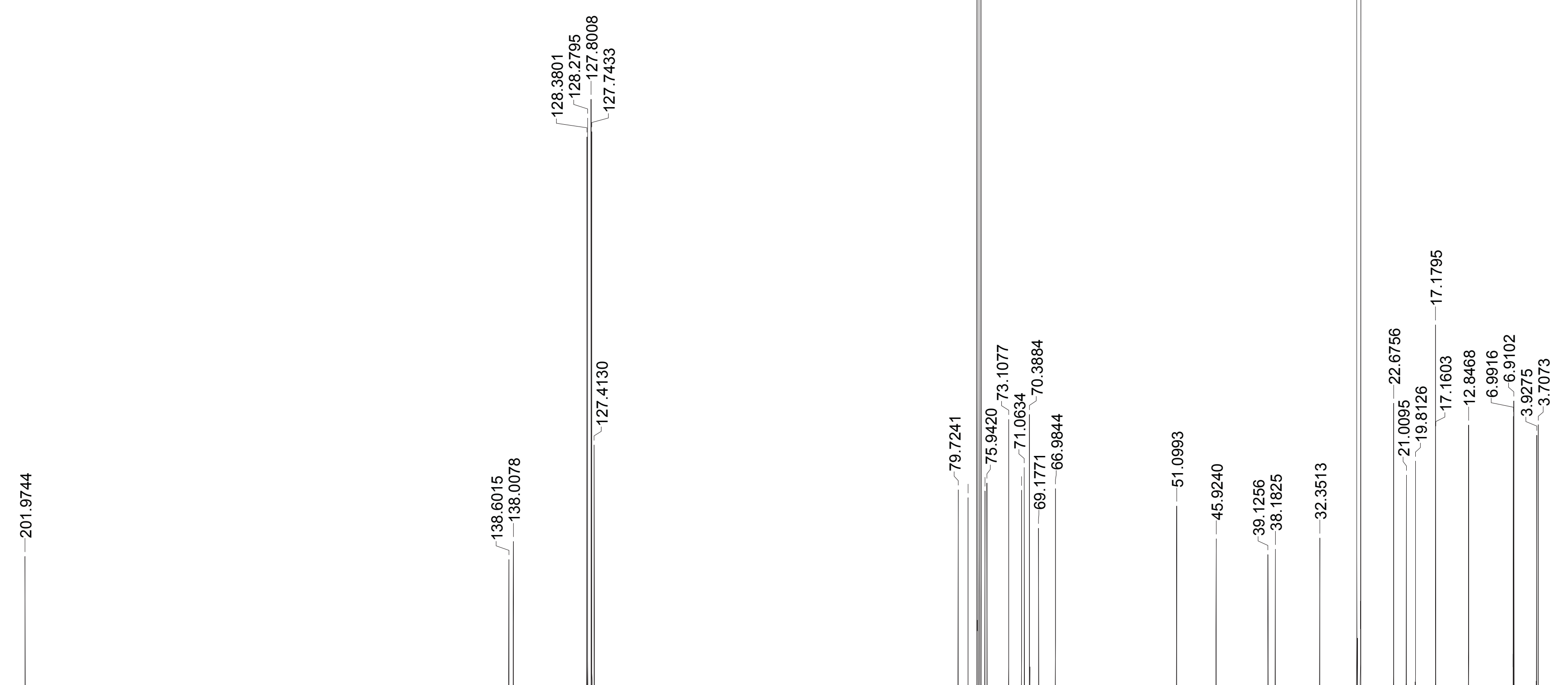

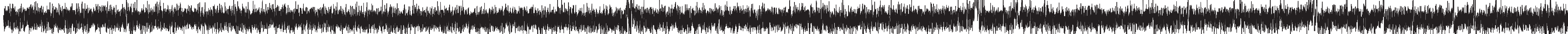


This report was created by ACD/NMR Processor Academic Edition. For more information go to www.acdlabs.com/nmrproc/

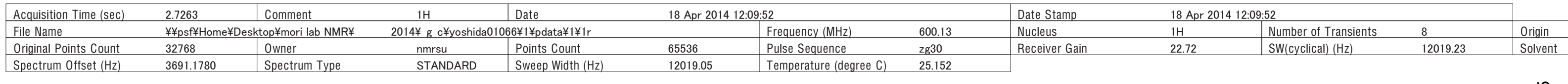

spect
CHLOROFORM-d

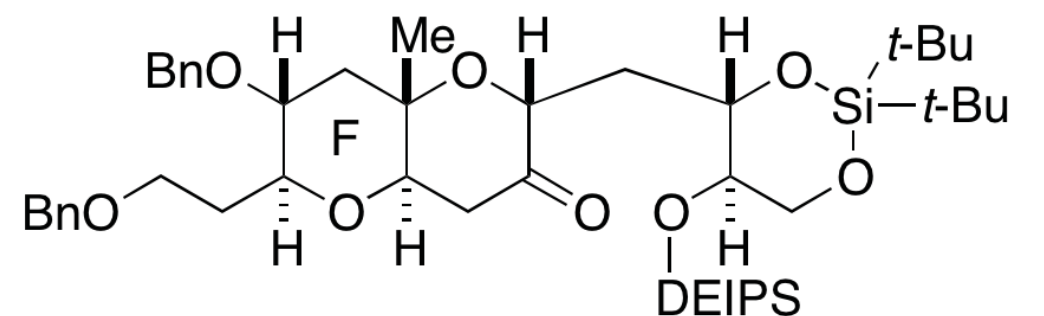

12

${ }^{1} \mathrm{H} \mathrm{NMR}\left(\mathrm{CDCl}_{3}, 600 \mathrm{MHz}\right)$
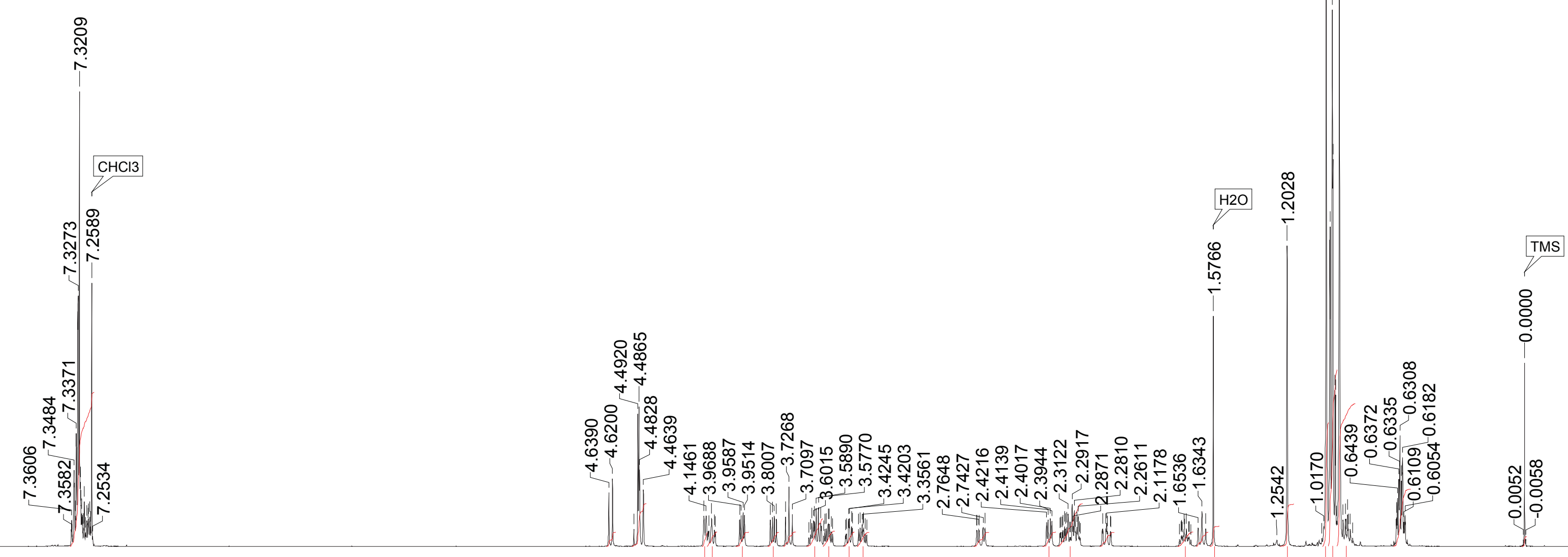

11.0

1.013.050.99 1.02 1.01 1.00 1.021.98 1.101.01 1.00

$1.00 \quad 1.013 .041 .00$

1.021 .011 .433 .018 .8912 .6510 .214 .10 
This report was created by ACD/NMR Processor Academic Edition. For more information go to www.acdlabs.com/nmrproc/

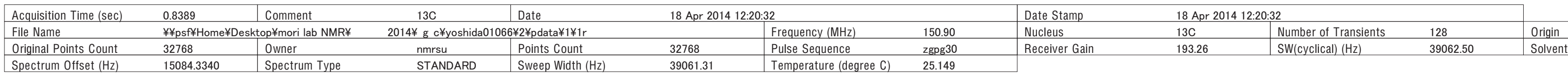

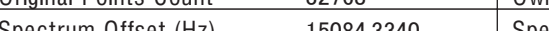
\begin{tabular}{l|ll}
32768 & Pullese Sequence & za.950 \\
39061.31 & Temperature (degree C) & 25.149 \\
& &
\end{tabular}

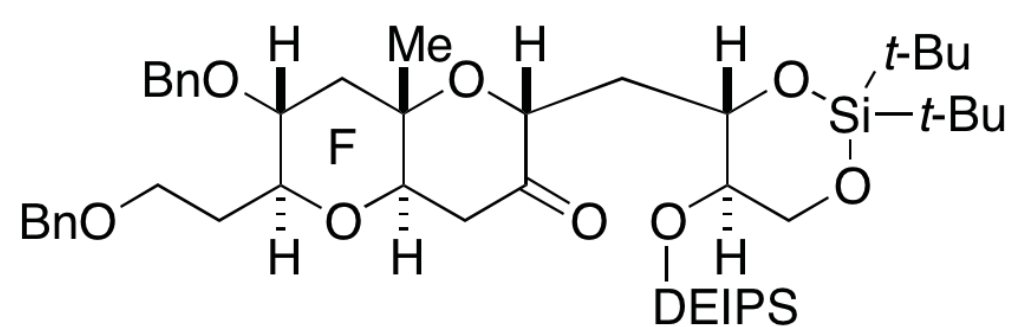

12

${ }^{13} \mathrm{C} \mathrm{NMR}\left(\mathrm{CDCl}_{3}, 150 \mathrm{MHz}\right)$
范客总

Кำ

怘

(ִ.)

总

1)

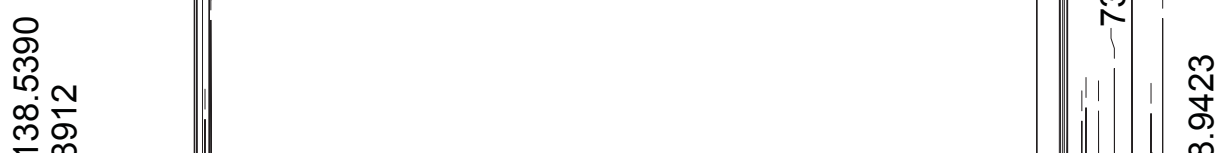

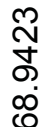

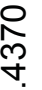

$\infty$
$\stackrel{8}{\circ}$
$\infty$
$\stackrel{0}{0}$

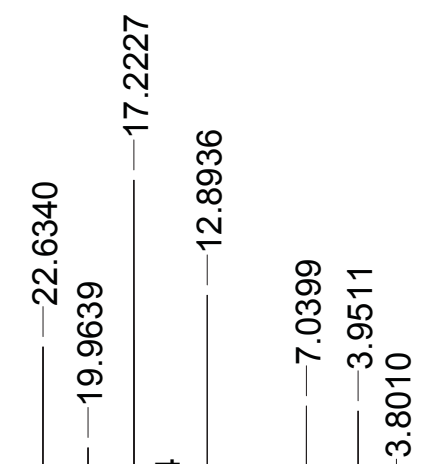




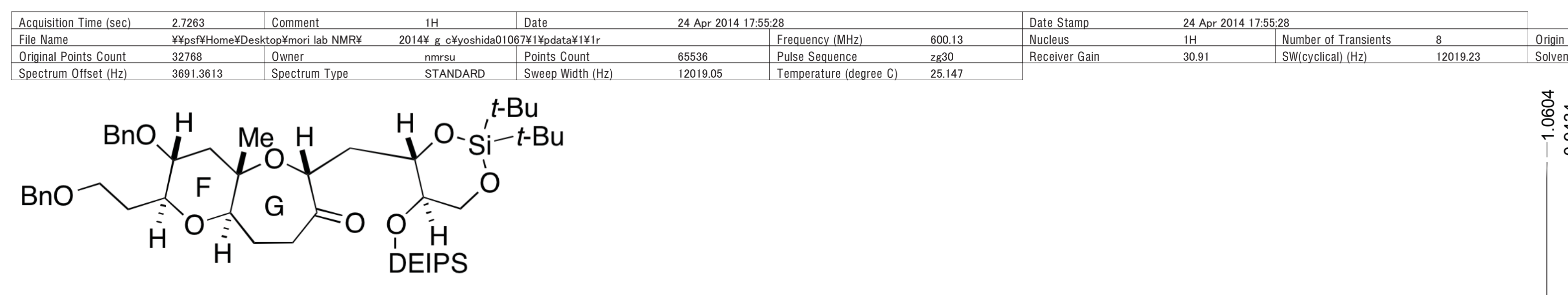

13

${ }^{1} \mathrm{H} \mathrm{NMR}\left(\mathrm{CDCl}_{3}, 600 \mathrm{MHz}\right)$
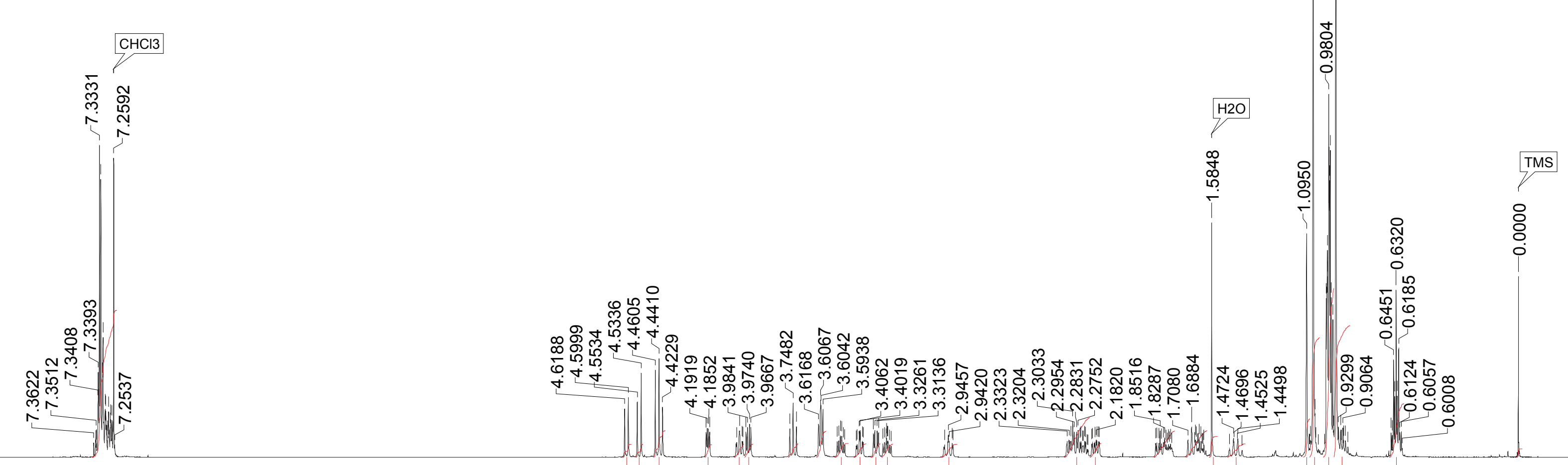

$\underbrace{11.03}_{7.5}$

0.970 .971 .990 .940 .990 .980 .991 .921 .030 .991 .000 .980 .96

2.951 .03

$951.98 \quad 1.560 .992 .898 .9712 .699 .894 .00$ 
This report was created by ACD/NMR Processor Academic Edition. For more information go to www.acdlabs.com/nmrproc/

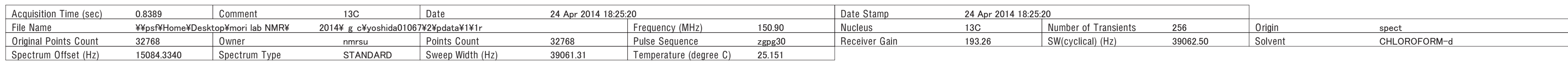

Spectrum Offset (Hz) 150843340

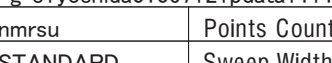

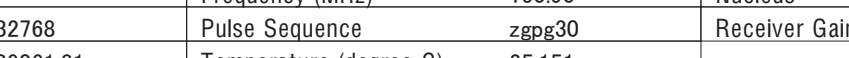
$\mathrm{CDCl} 3$

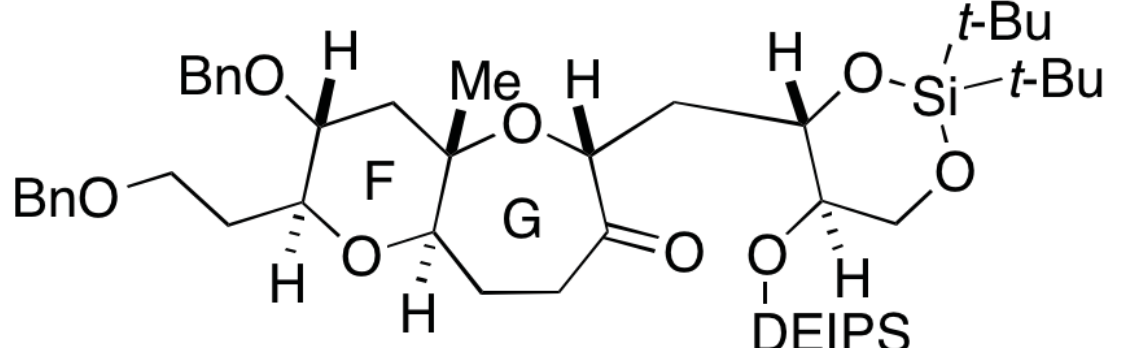

13

${ }^{13} \mathrm{C} \mathrm{NMR}\left(\mathrm{CDCl}_{3}, 150 \mathrm{MHz}\right)$

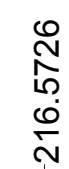

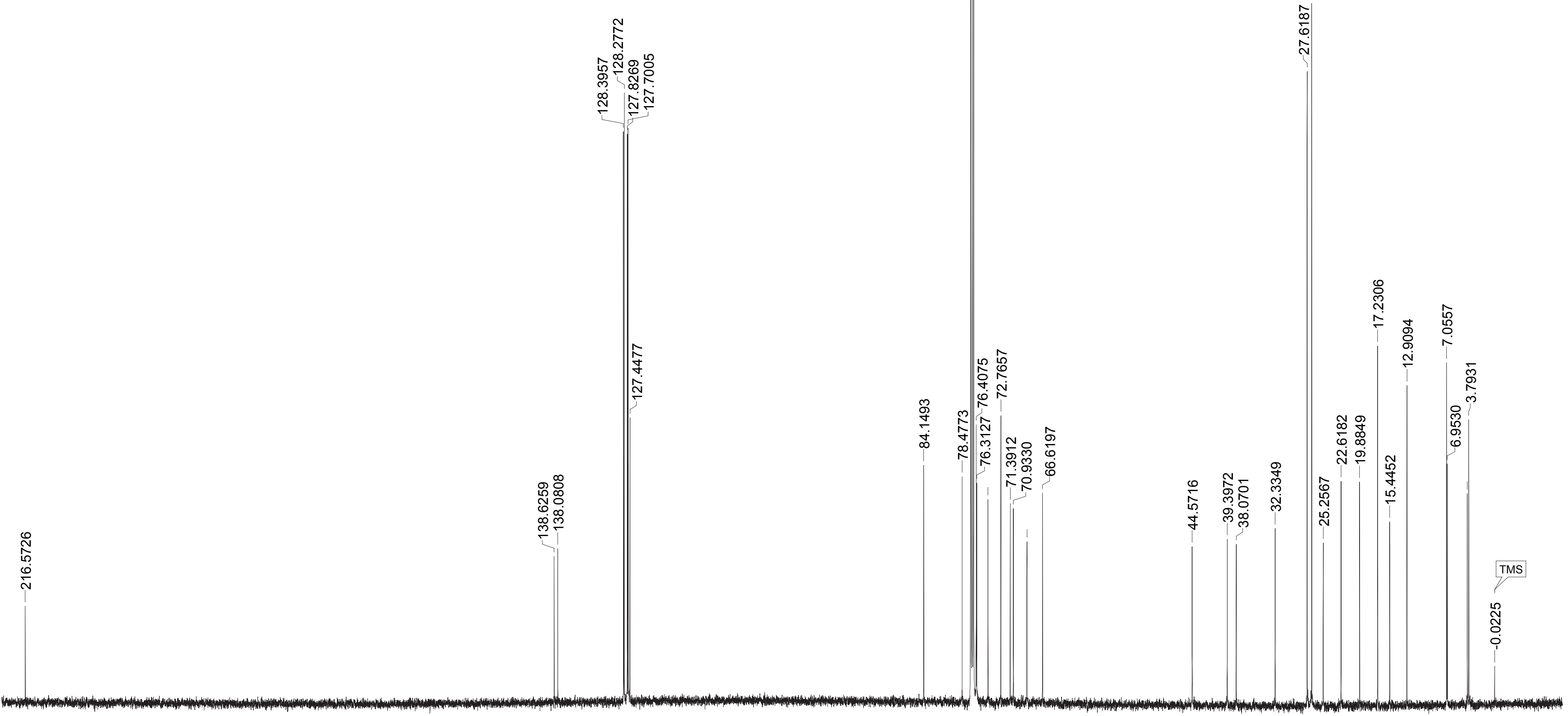

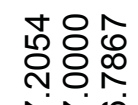

Кำ 
This report was created by ACD/NMR Processor Academic Edition. For more information go to www.acdlabs.com/nmrproc/

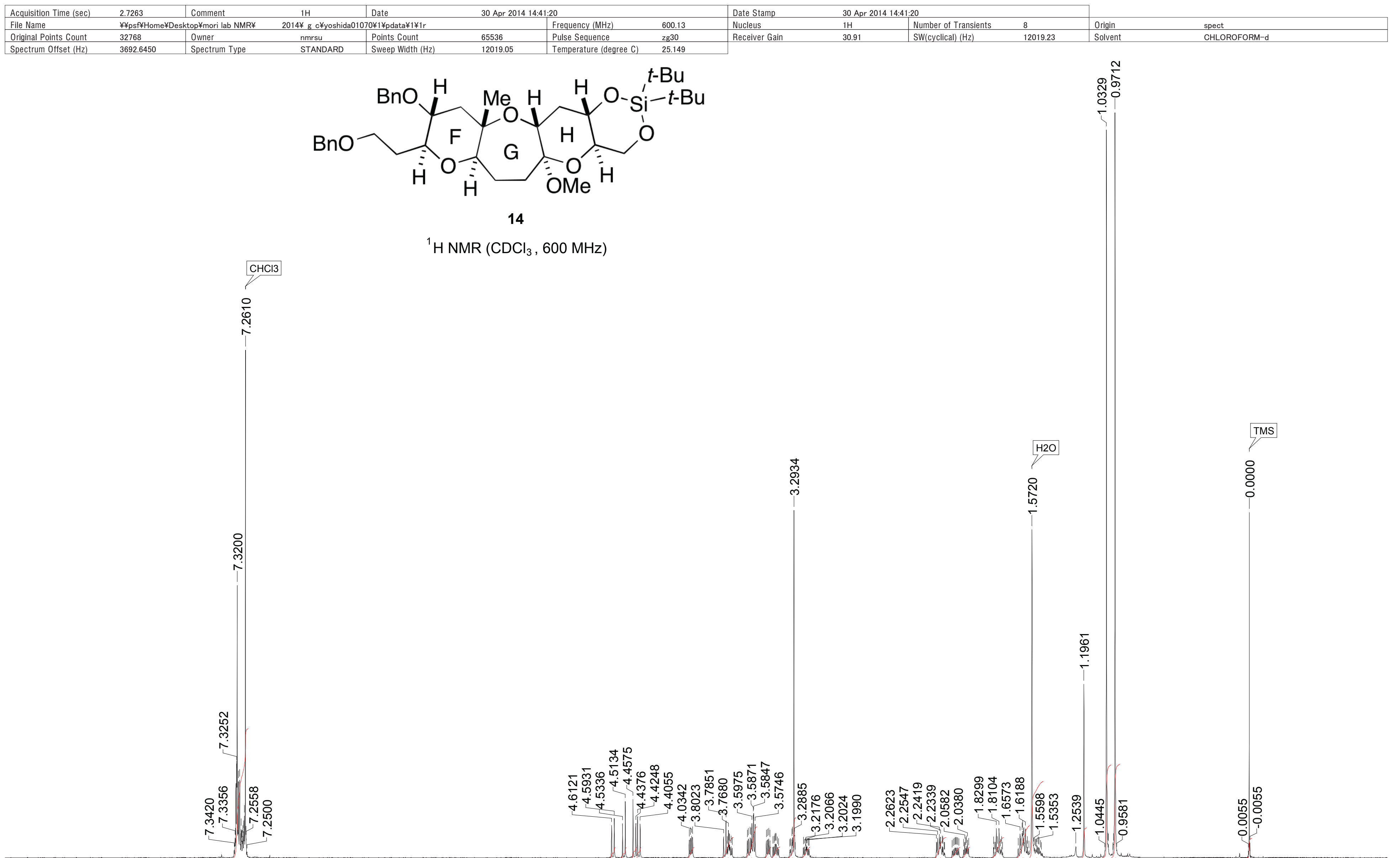

12.86

$1.001 .001 .021 .00 \quad 1.00 \quad 2.023 .061 .021 .054 .001 .06$

$2.021 .011 .042 .013 .217 .61-2.979 .269 .33$

1.91 
This report was created by ACD/NMR Processor Academic Edition. For more information go to www.acdlabs.com/nmrproc/

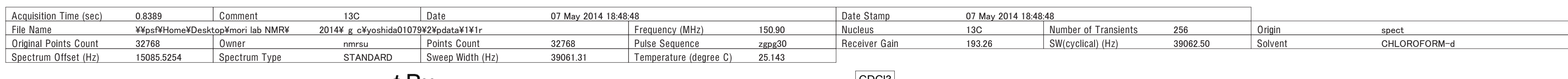

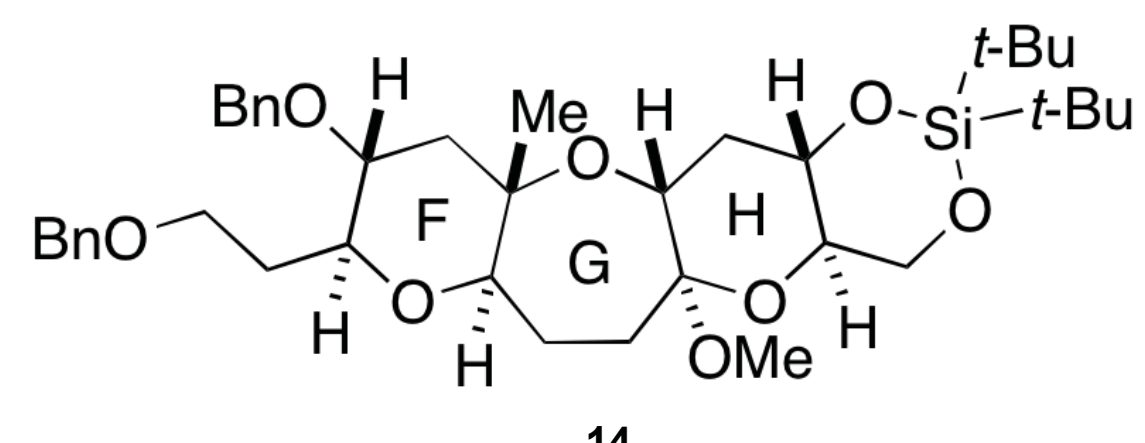

${ }^{13} \mathrm{CNMR}\left(\mathrm{CDCl}_{3}, 150 \mathrm{MHz}\right)$

$\underset{\substack{\check{\infty} \\ \stackrel{\Gamma}{\sim}}}{\stackrel{\Gamma}{\sim}}$

공

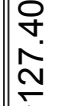

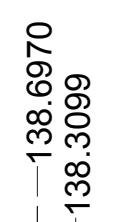

$\mathrm{CDCl} 3$

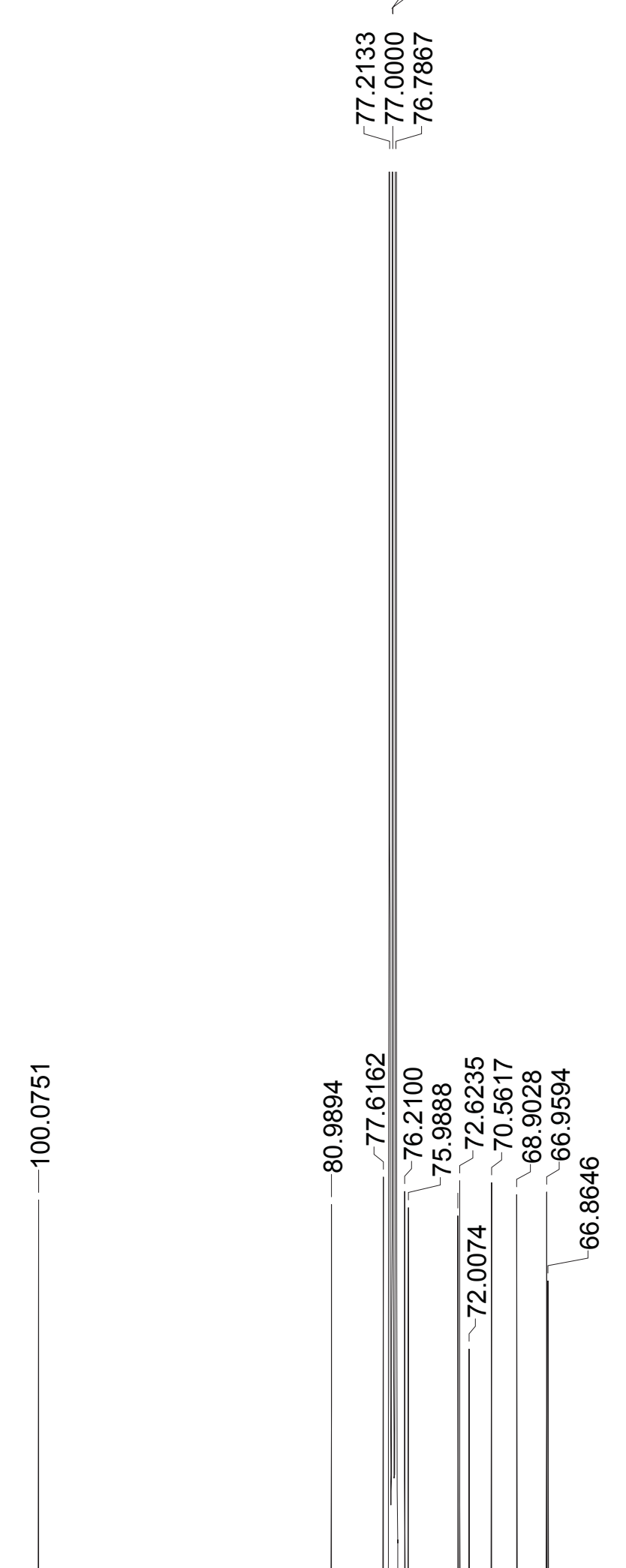

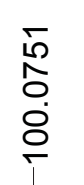

TMS 


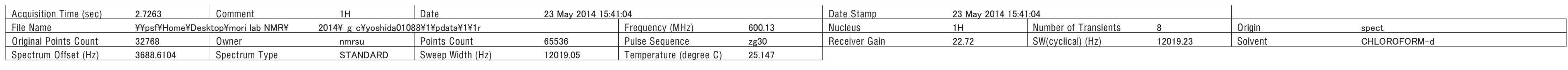

(-

${ }^{1} \mathrm{H} \mathrm{NMR}\left(\mathrm{CDCl}_{3}, 600 \mathrm{MHz}\right)$ 
This report was created by ACD/NMR Processor Academic Edition. For more information go to www.acdlabs.com/nmrproc/

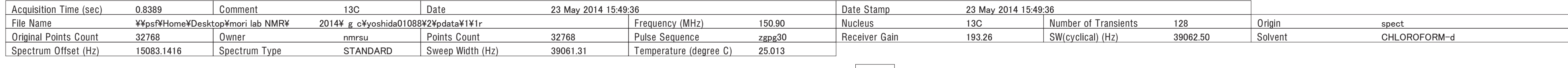



15

${ }^{13} \mathrm{C} \mathrm{NMR}\left(\mathrm{CDCl}_{3}, 150 \mathrm{MHz}\right)$
$\mathrm{CDCl} 3$

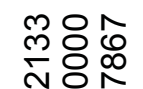

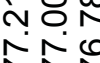

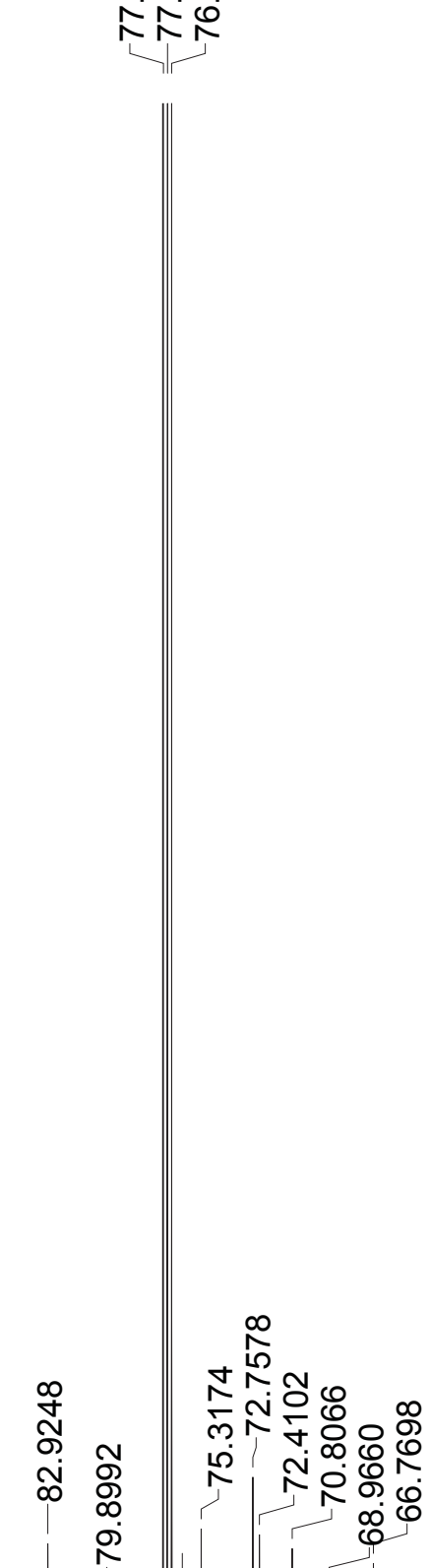


This report was created by ACD/NMR Processor Academic Edition. For more information go to www.acdlabs.com/nmrproc/



12.39

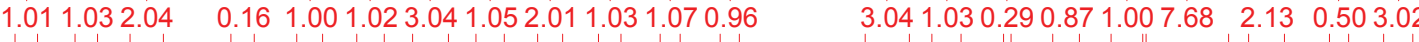

1.61 
This report was created by ACD/NMR Processor Academic Edition. For more information go to www.acdlabs.com/nmrproc/

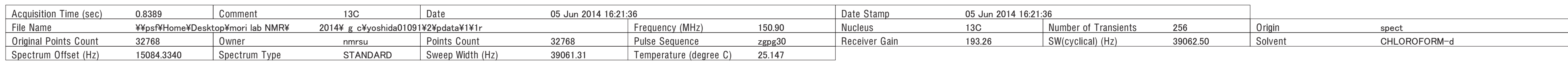

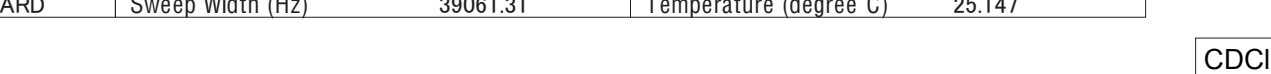

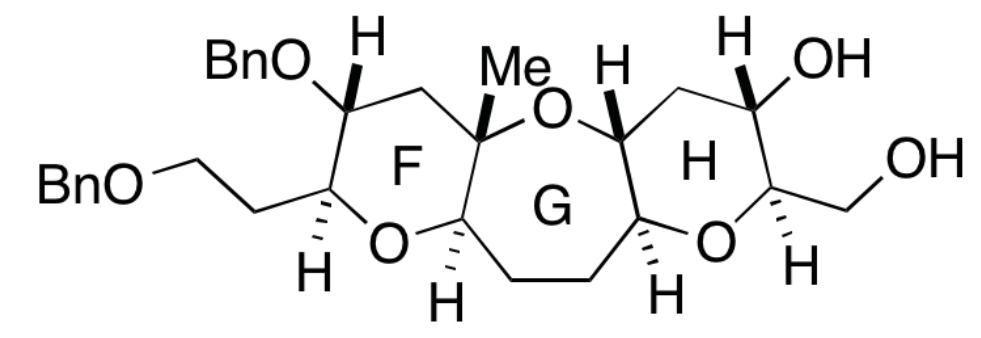

S6

${ }^{13} \mathrm{C} \mathrm{NMR}\left(\mathrm{CDCl}_{3}, 150 \mathrm{MHz}\right)$

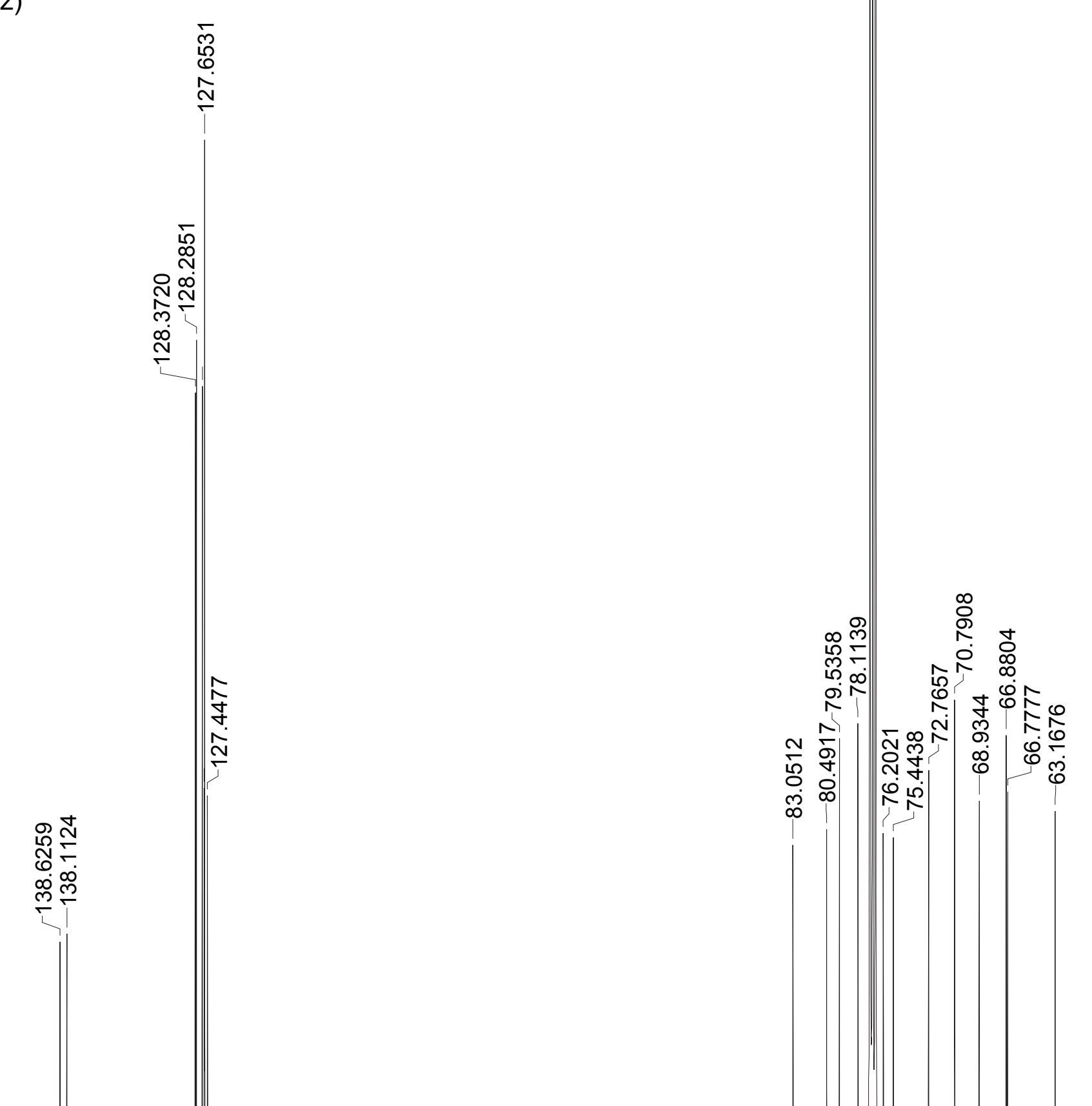

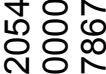

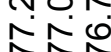



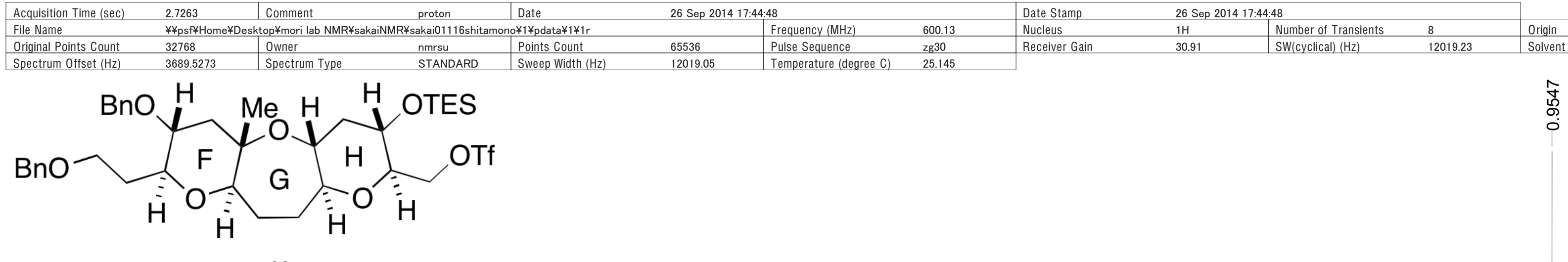

16

${ }^{1} \mathrm{H} \mathrm{NMR}\left(\mathrm{CDCl}_{3}, 600 \mathrm{MHz}\right)$

$\stackrel{\text { ্ָ }}{\stackrel{N}{*}}$

$\mathrm{CHCl} 3$

$\underset{\substack{\infty \\ \stackrel{\infty}{N}}}{\stackrel{1}{N}}$

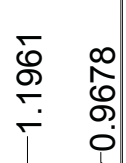

เับ

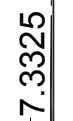

ำ

趈

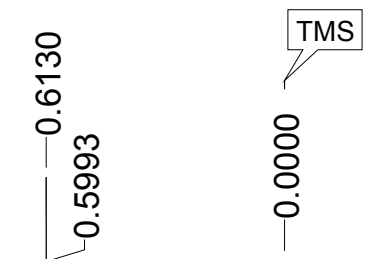

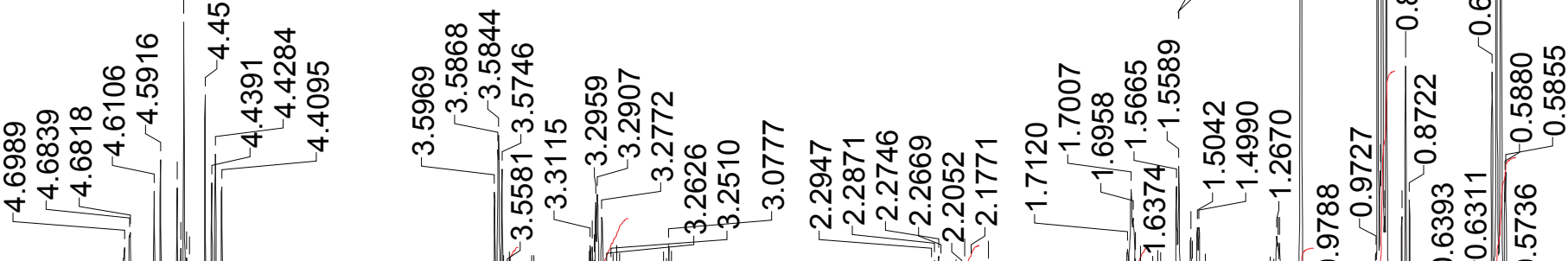

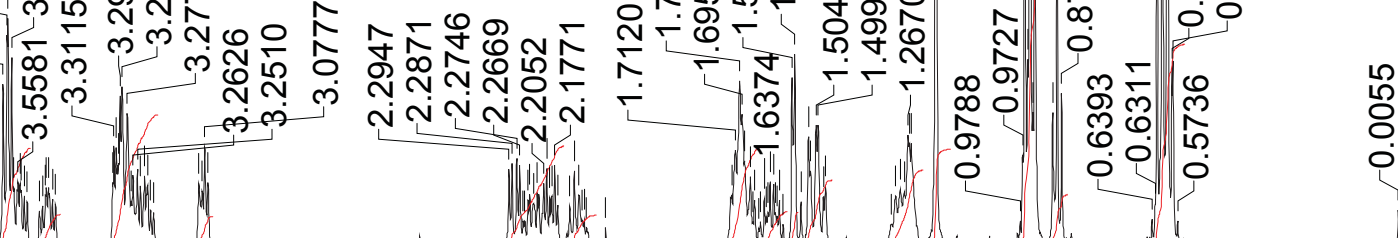

2.971 .003 .940 .95

$3.020 .98 \quad 2.991 .091 .072 .012 .322 .938 .981 .60 \quad 6.03$

0.970 .971 .961 .020 .98

$3.5 \quad 3.0$

2.5

1.5

$1.0 \quad 0.5$ 
This report was created by ACD/NMR Processor Academic Edition. For more information go to www.acdlabs.com/nmrproc/

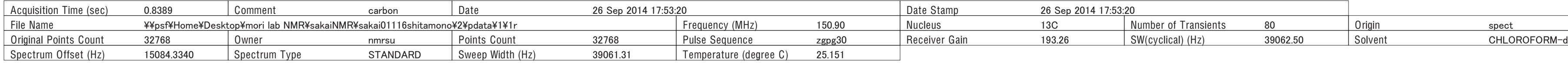

Orignal Points Count

STANDARD SW SW

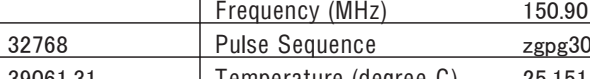
$\mathrm{CDCl3}$

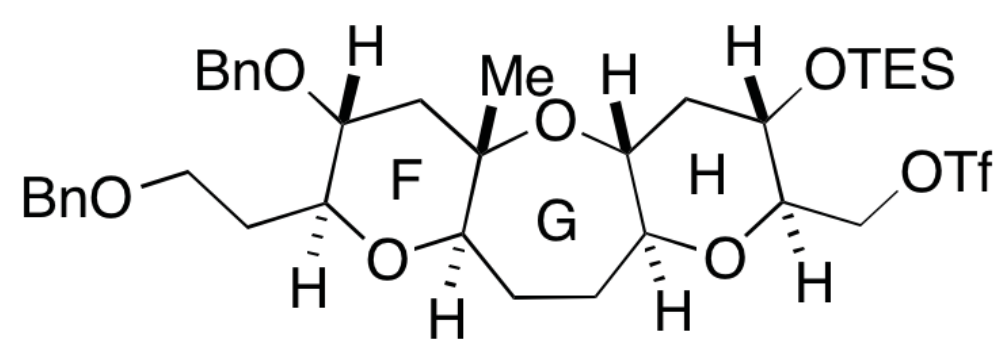

16

${ }^{13} \mathrm{C} \mathrm{NMR}\left(\mathrm{CDCl}_{3}, 150 \mathrm{MHz}\right)$

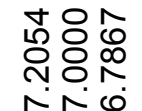

ลำ

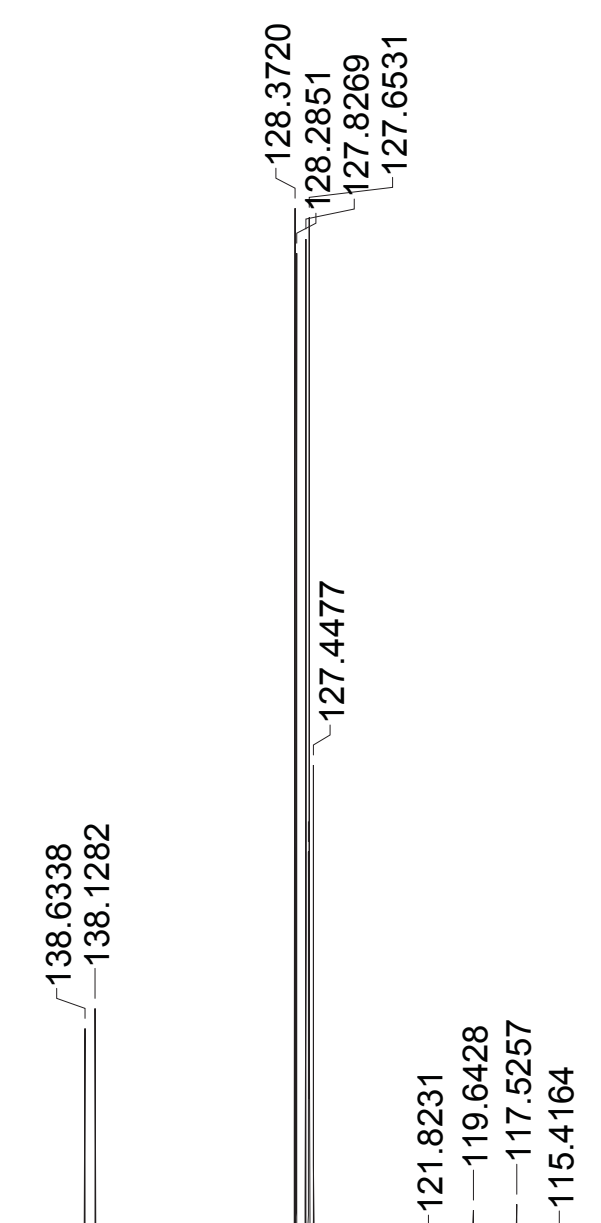

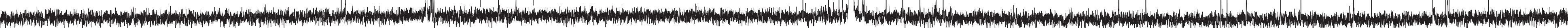

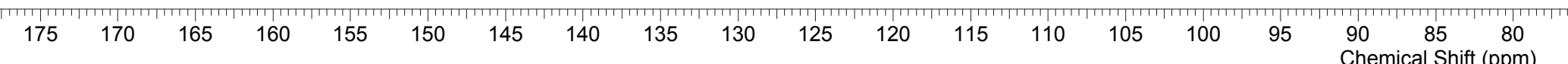




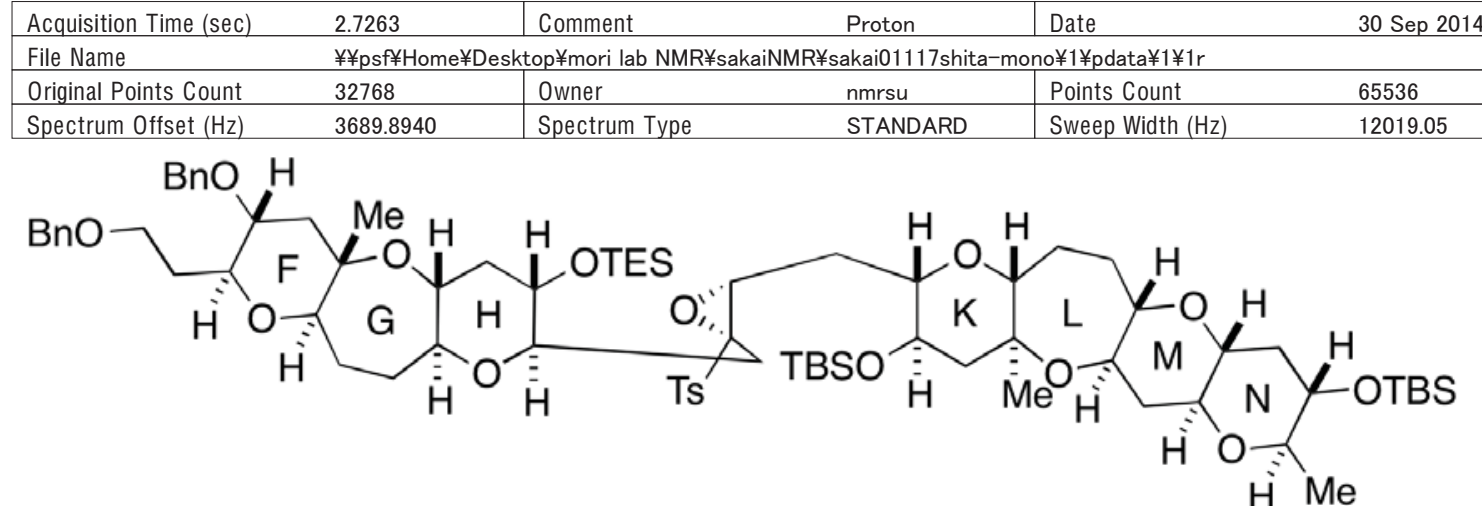

$17(\mathrm{dr}=94: 6)$

${ }^{1} \mathrm{H}$ NMR $\left(\mathrm{CDCl}_{3}, 600 \mathrm{MHz}\right)$

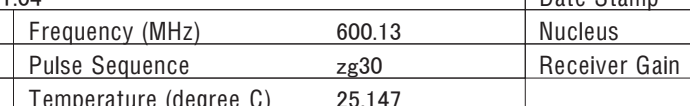
\begin{tabular}{ll} 
Frequency (MH & 600.13 \\
Pulse esequence & 2830 \\
\hline Temperature (degree C) & 25.147 \\
\hline
\end{tabular} 22.72 \begin{tabular}{|l}
\hline Number of Transients \\
\hline SW(cyclical) (Hz) \\
\hline
\end{tabular}

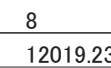
8

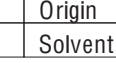
spect
CHLOROFORM-d
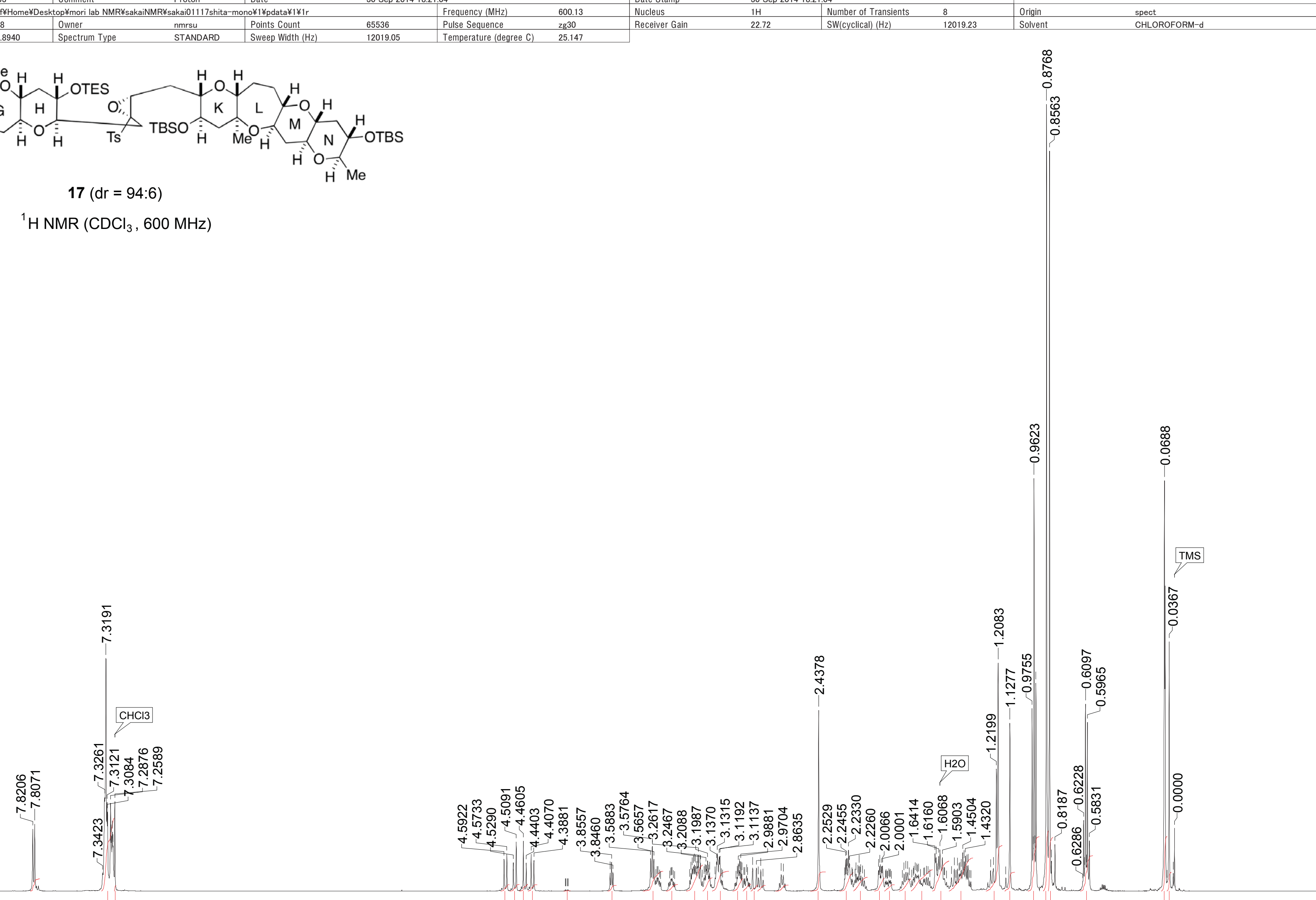

1.02 1.06 1.05 1.04 0.06 0.94 3.22 1.06 4.27 2.183.23 2.02 1.08 0.96 0.940.963.14 3.02 2.27 2.11 1.05 2.00 3.48 4.90 5.397.44 3.08 8.88 9.79 8.76 5.76 $\quad 8.923 .07$ 


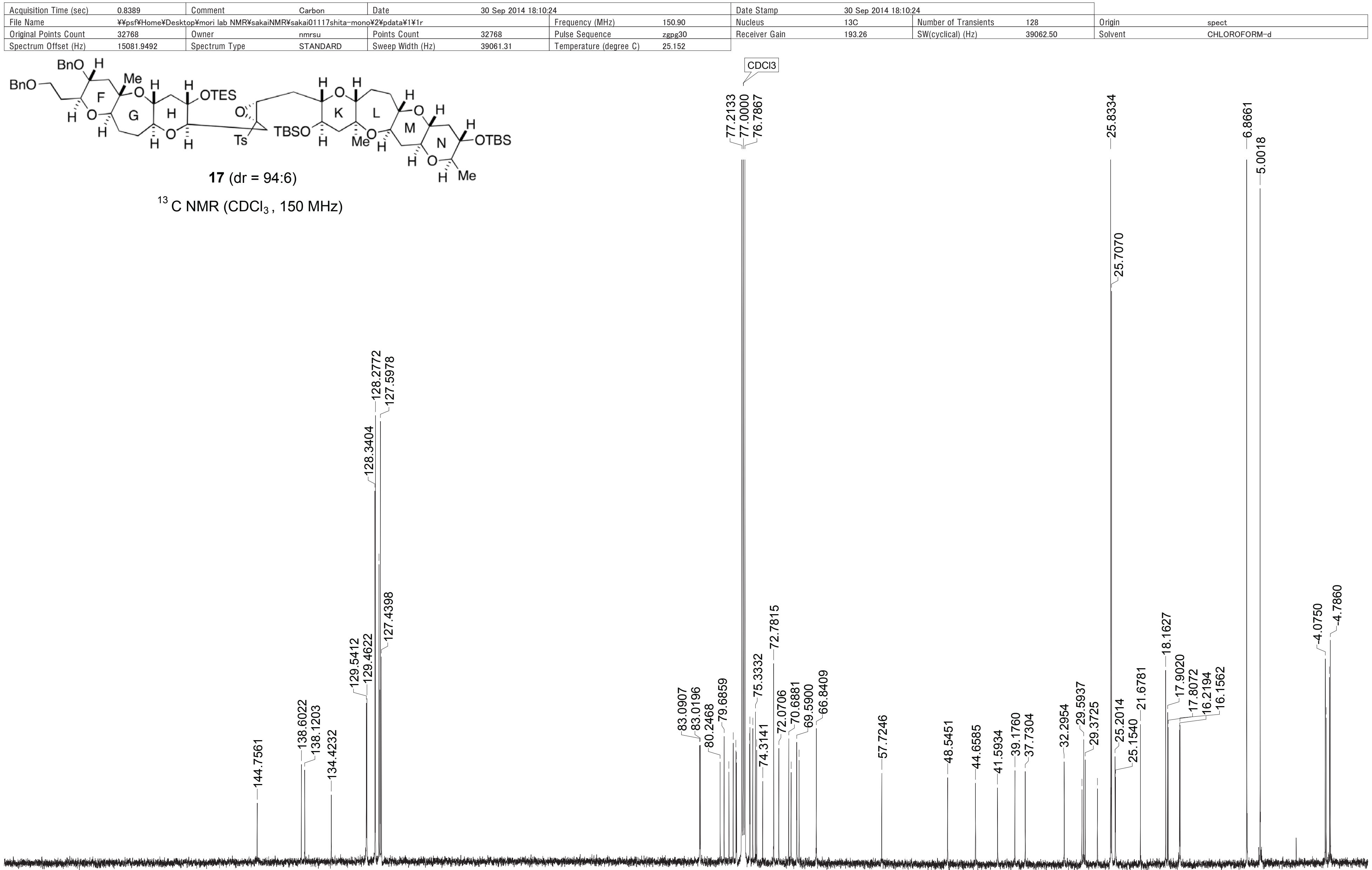






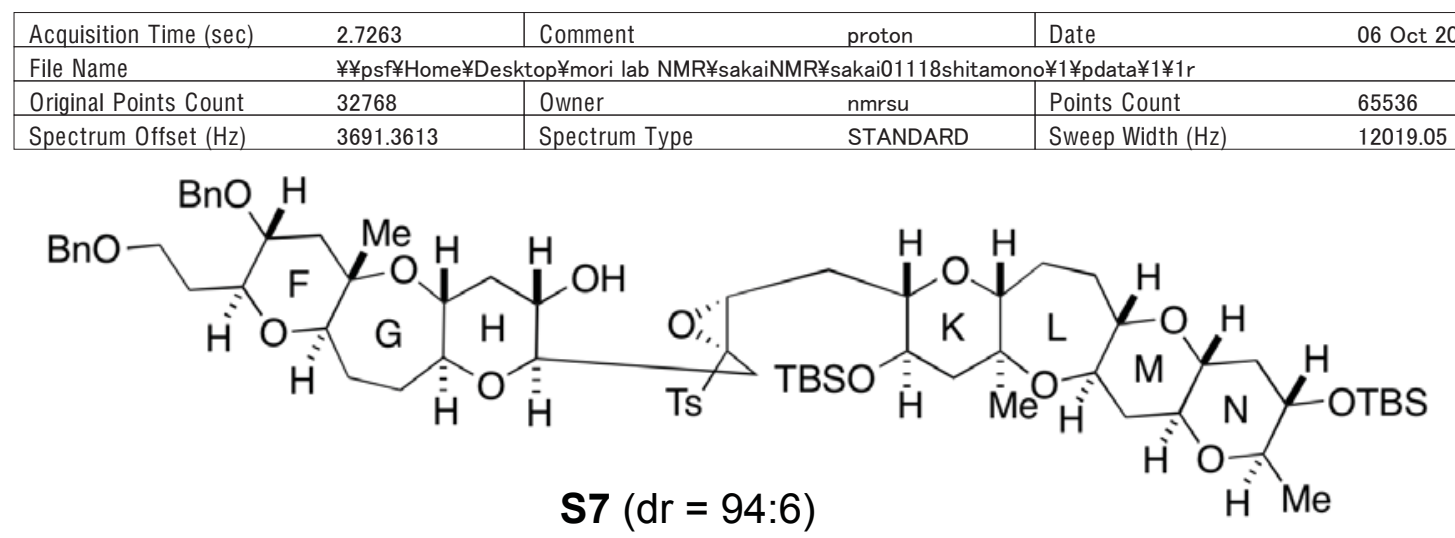

S7 $(\mathrm{dr}=94: 6)$

${ }^{1} \mathrm{H} \operatorname{NMR}\left(\mathrm{CDCl}_{3}, 600 \mathrm{MHz}\right)$

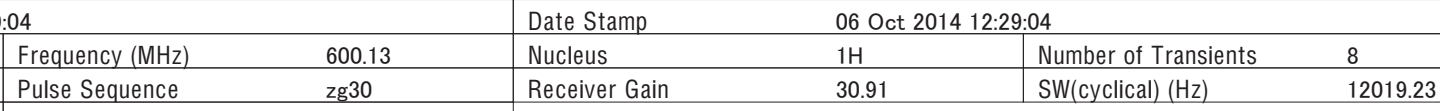

\begin{tabular}{|l|l} 
Origin \\
Solvent
\end{tabular}

spect
CHLOROFORM-d



$2.03 \quad 14.66$

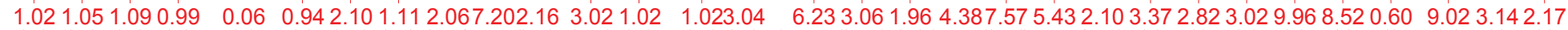




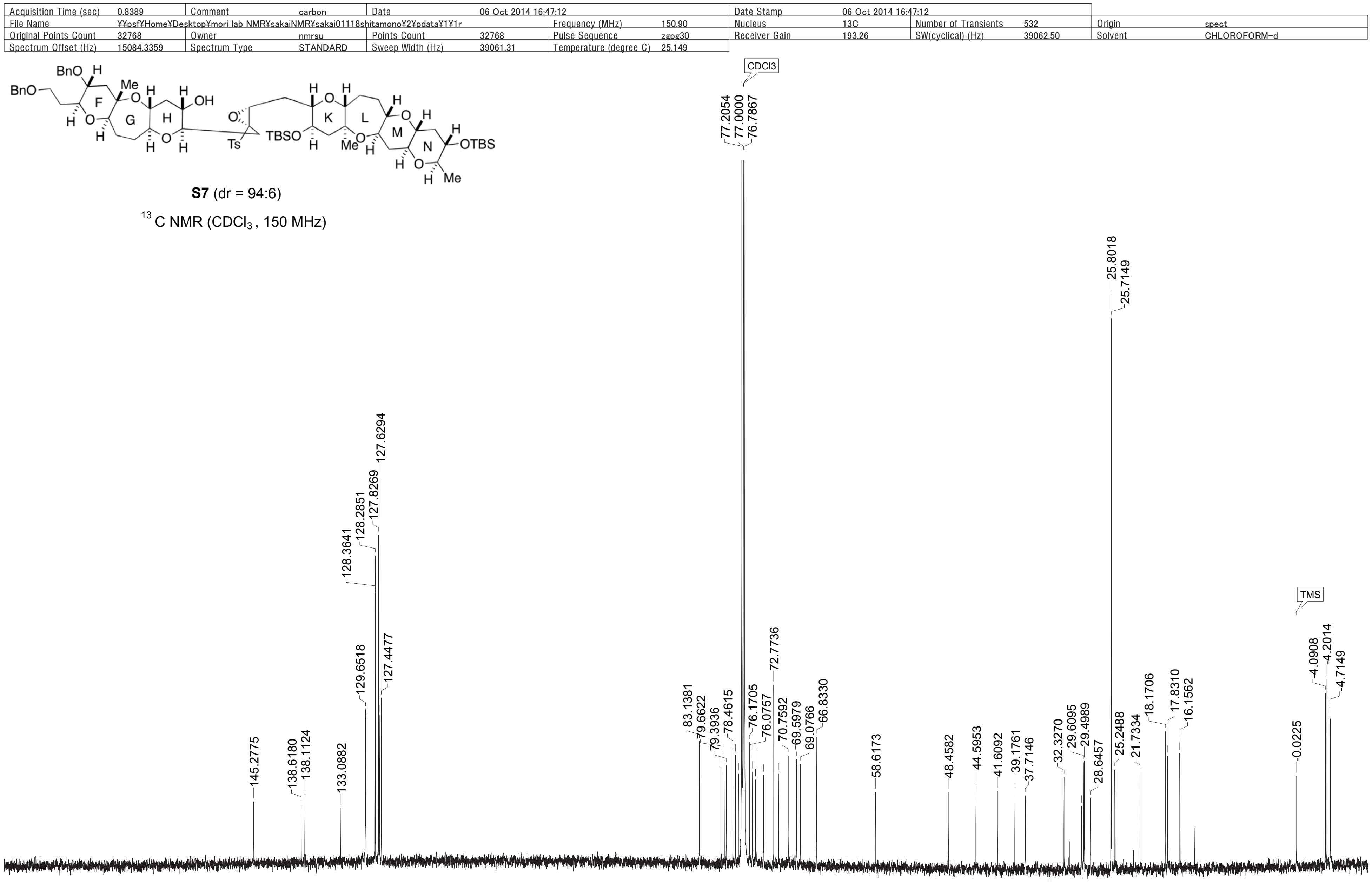




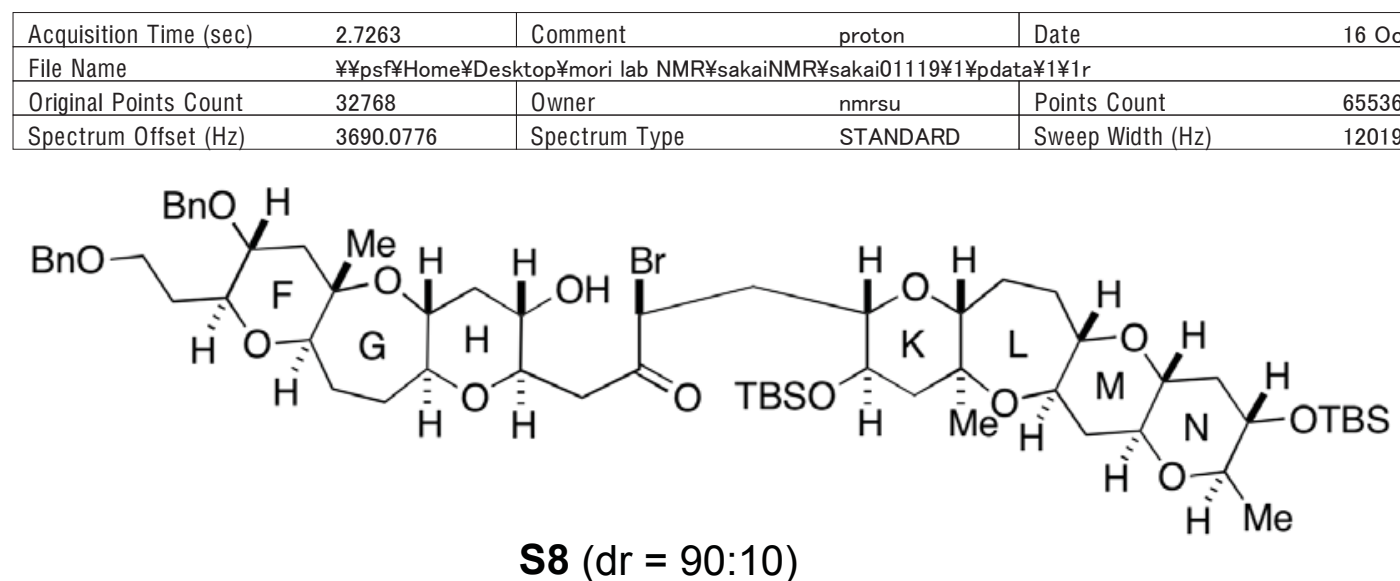

S8 $(\mathrm{dr}=90: 10)$

${ }^{1} \mathrm{H}$ NMR $\left(\mathrm{CDCl}_{3}, 600 \mathrm{MHz}\right)$

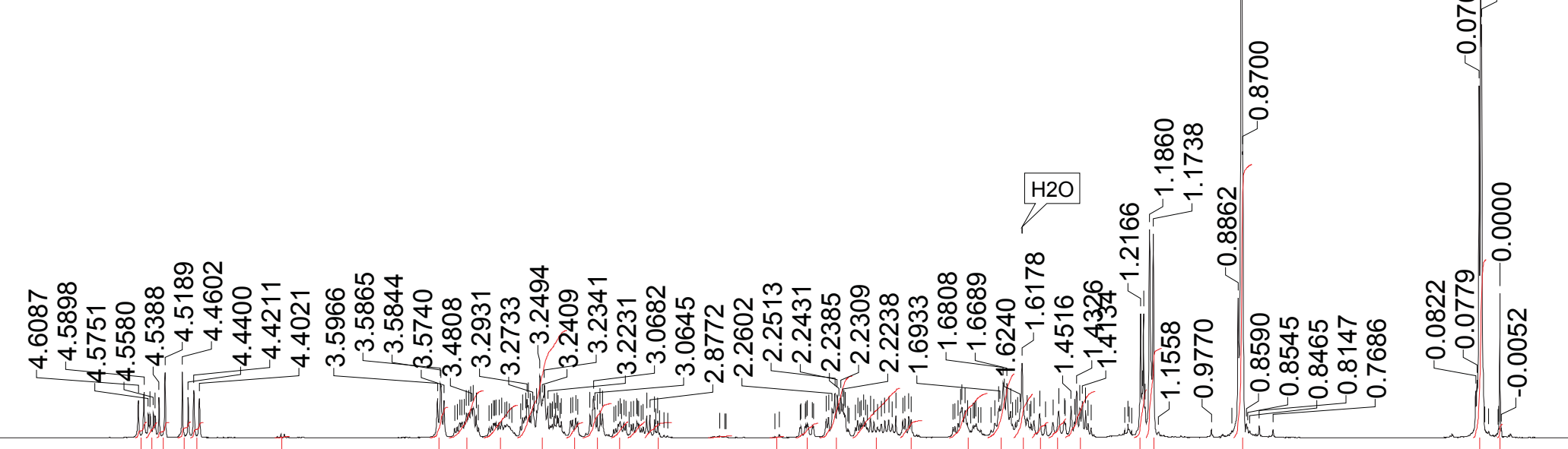

0.97 0.93 0.96 0.98 0.94 0.07 1.91 2.93 2.04 6.72 0.95 2.150.98 1.000.960.10 0.06 0.90 3.87 3.10 1.08 2.78 4.01 2.72 0.96 1.17 2.90 3.00 5.59 17.13 11.150 .85 


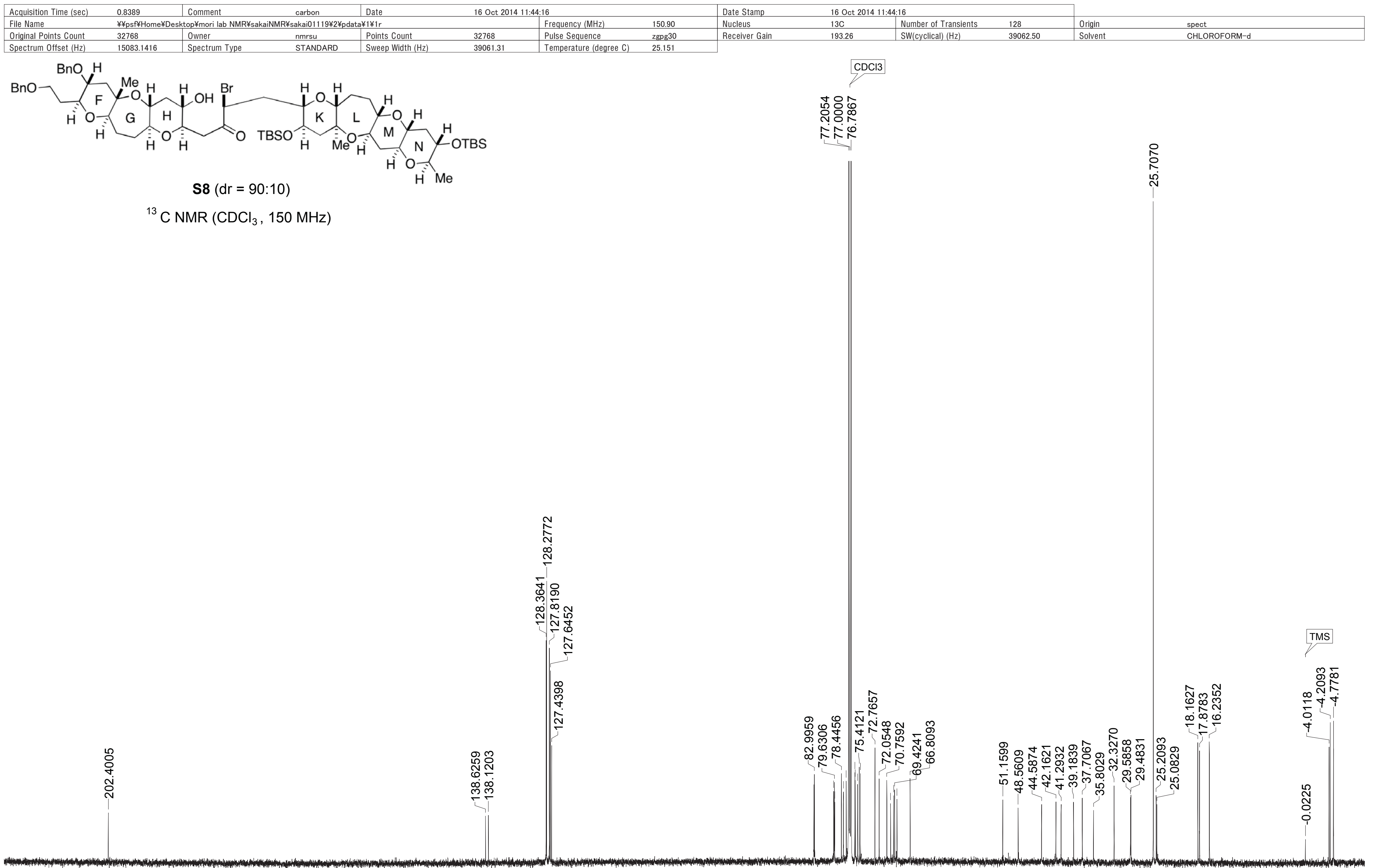



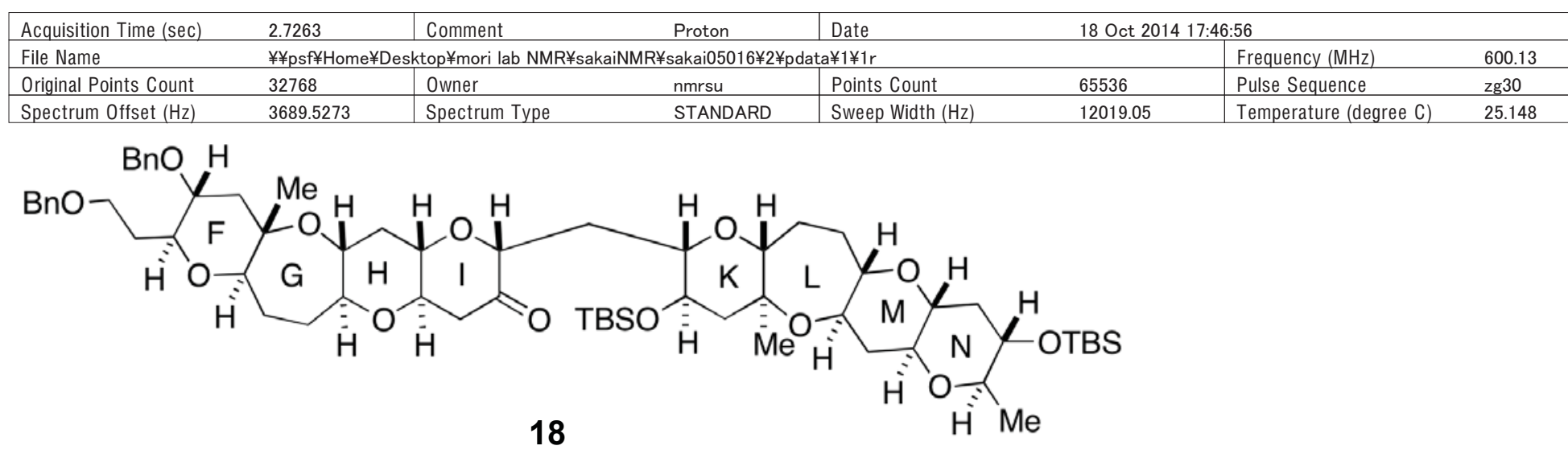

\section{${ }^{1} \mathrm{H} \mathrm{NMR}\left(\mathrm{CDCl}_{3}, 600 \mathrm{MHz}\right)$}
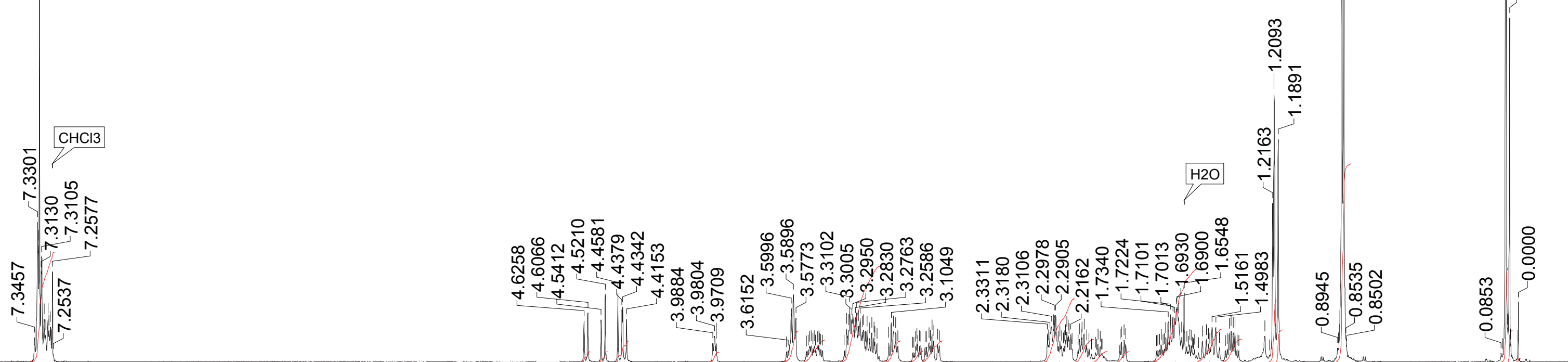

10.85

$\begin{array}{llll}1.011 .022 .07 & 0.98 & 3.072 .16 & 9.48 \\ 2.091 .072 .07\end{array}$

$6.242 .261 .051 .009 .213 .232 .266 .213 .18 \quad 19.57$

9.403 .120 .38 


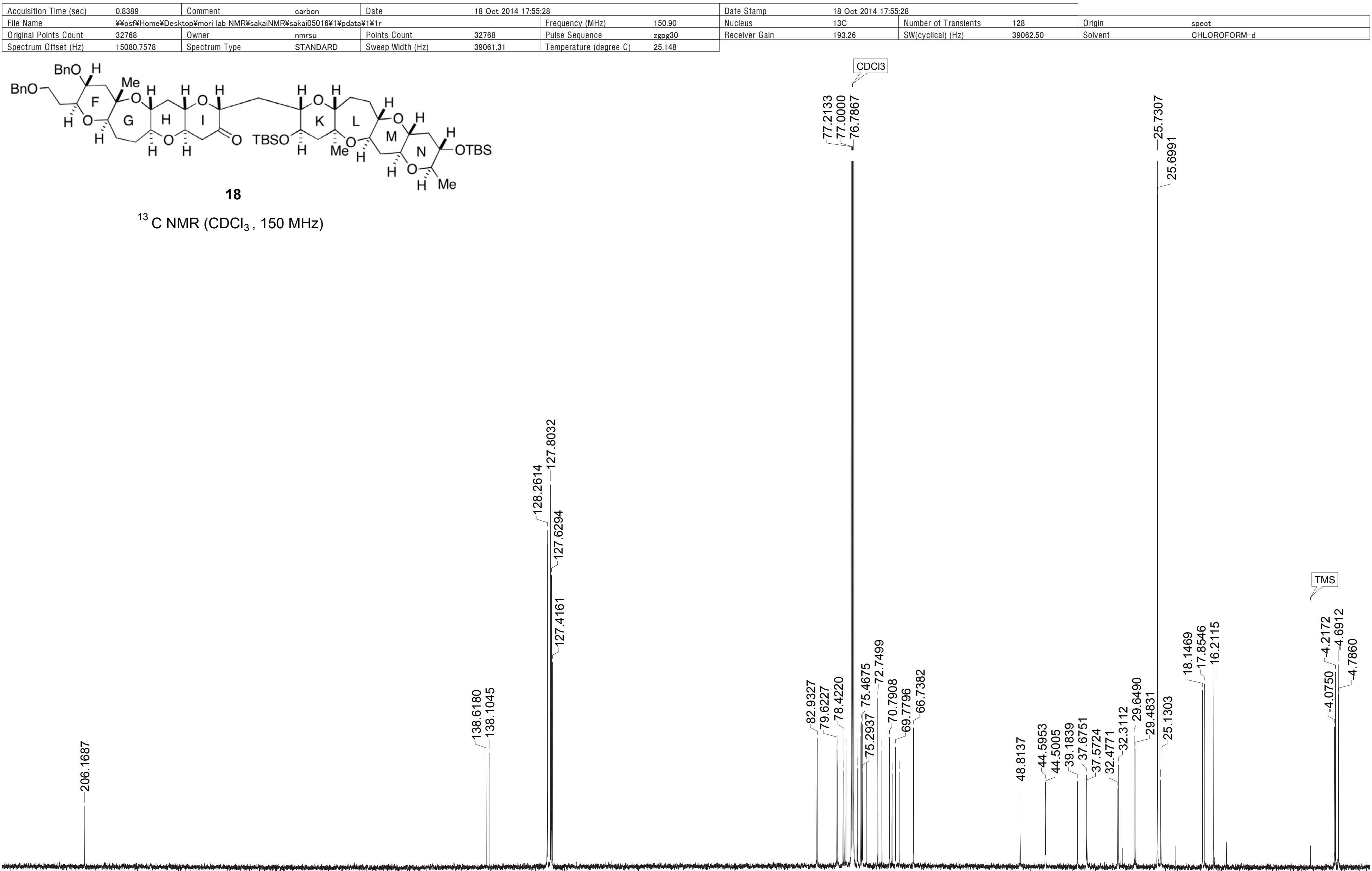




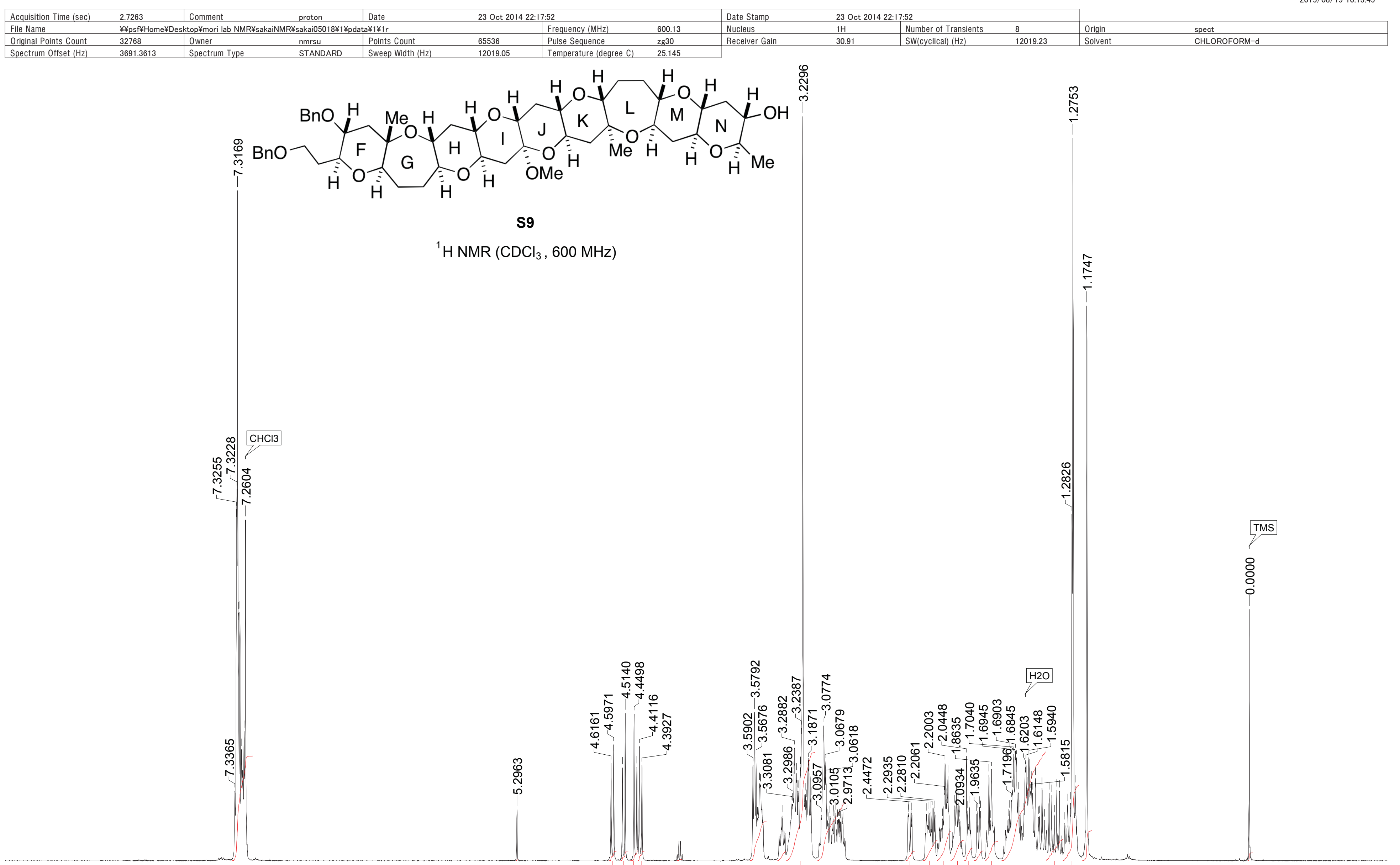




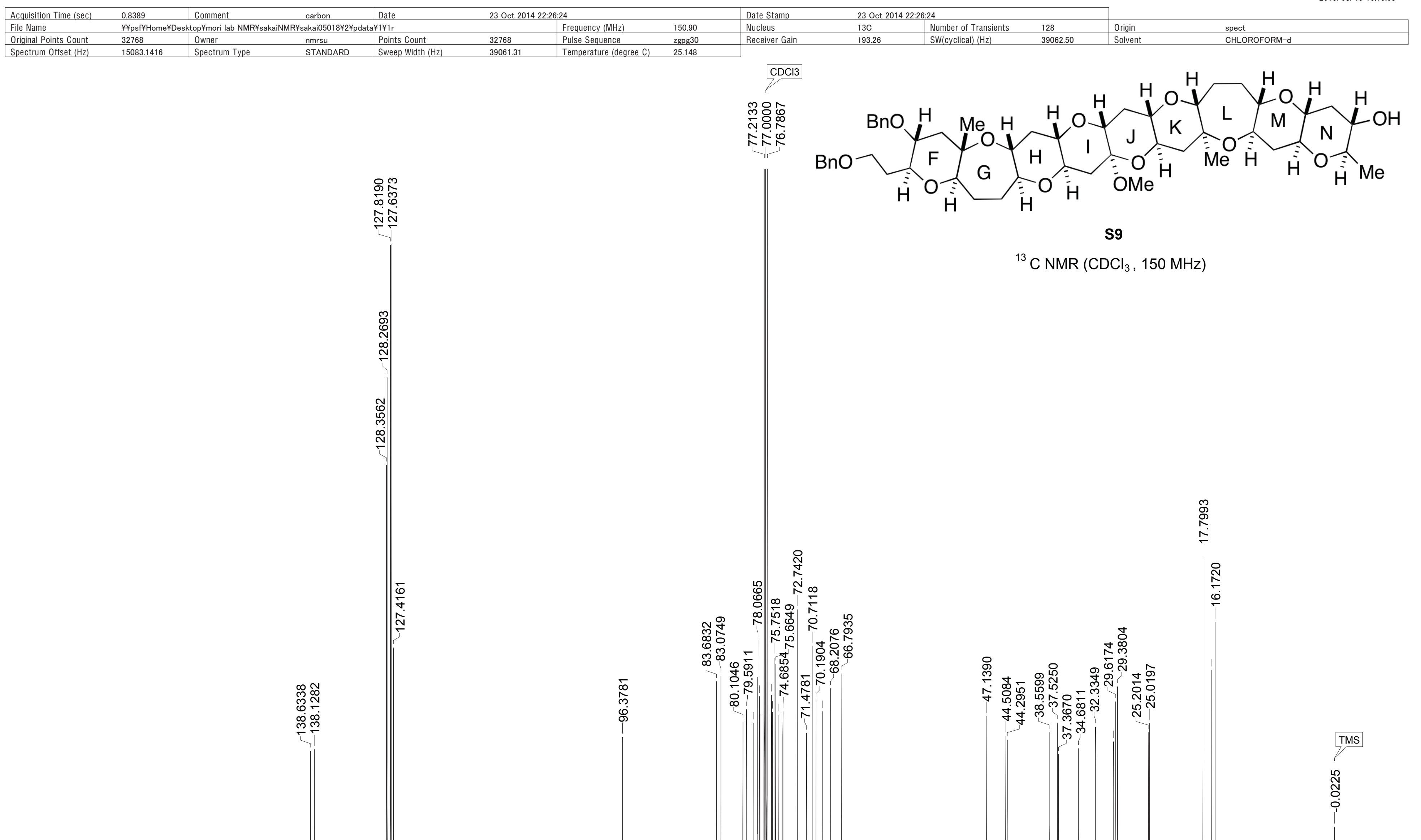




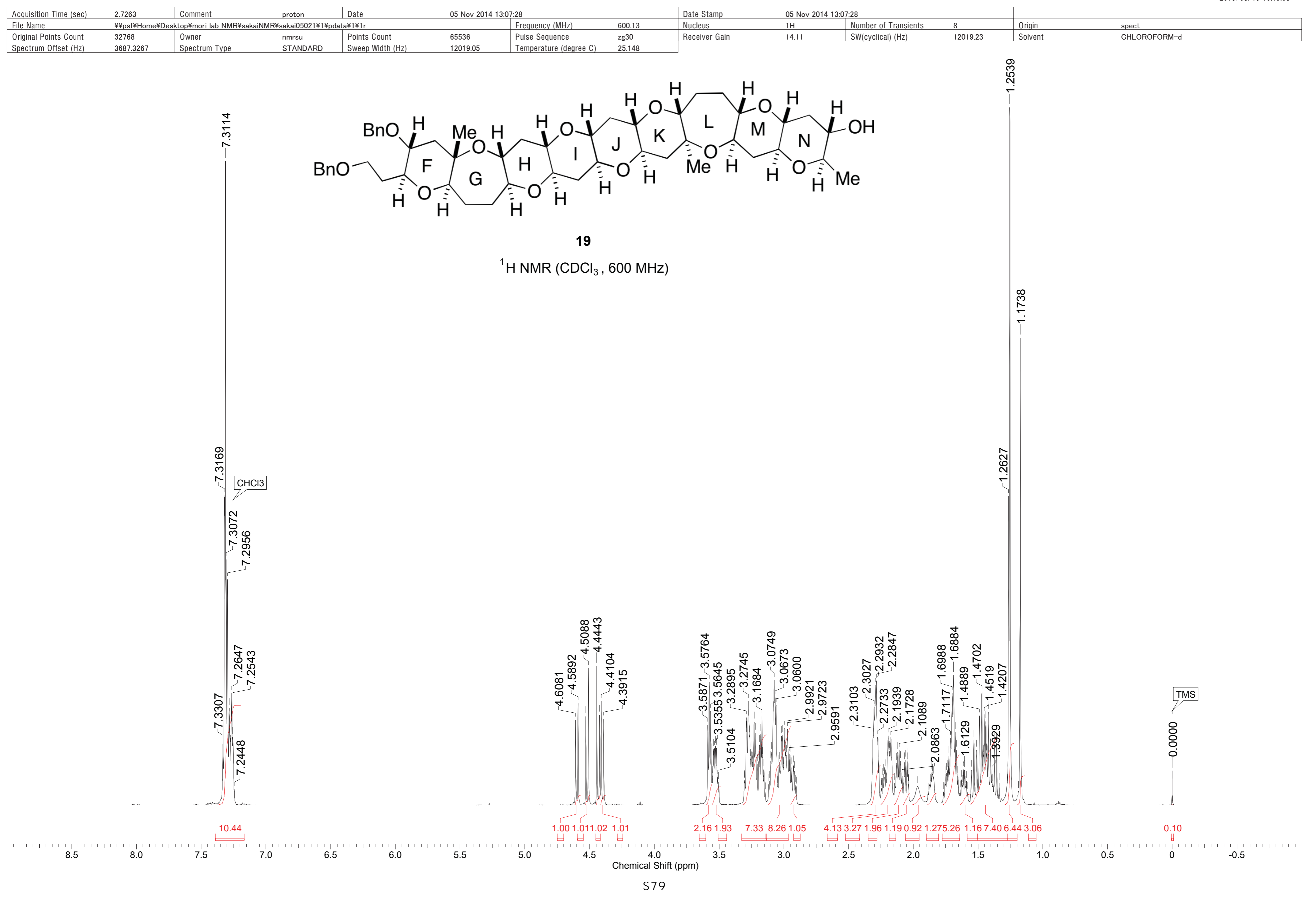




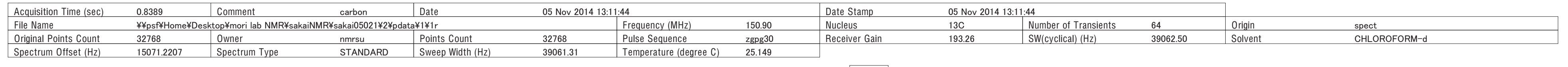

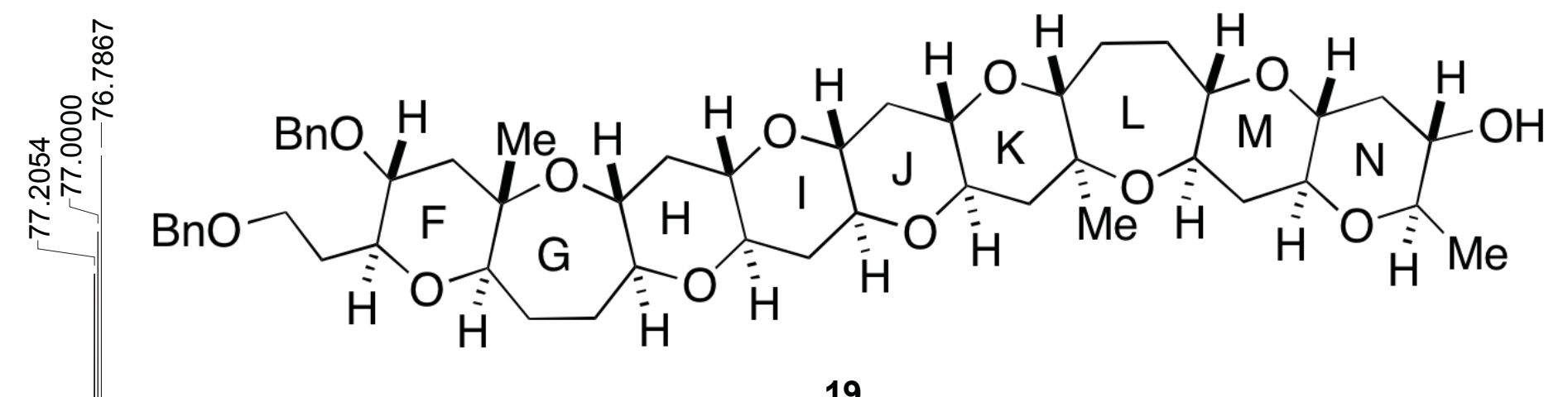

${ }^{13} \mathrm{C} \mathrm{NMR}\left(\mathrm{CDCl}_{3}, 150 \mathrm{MHz}\right)$
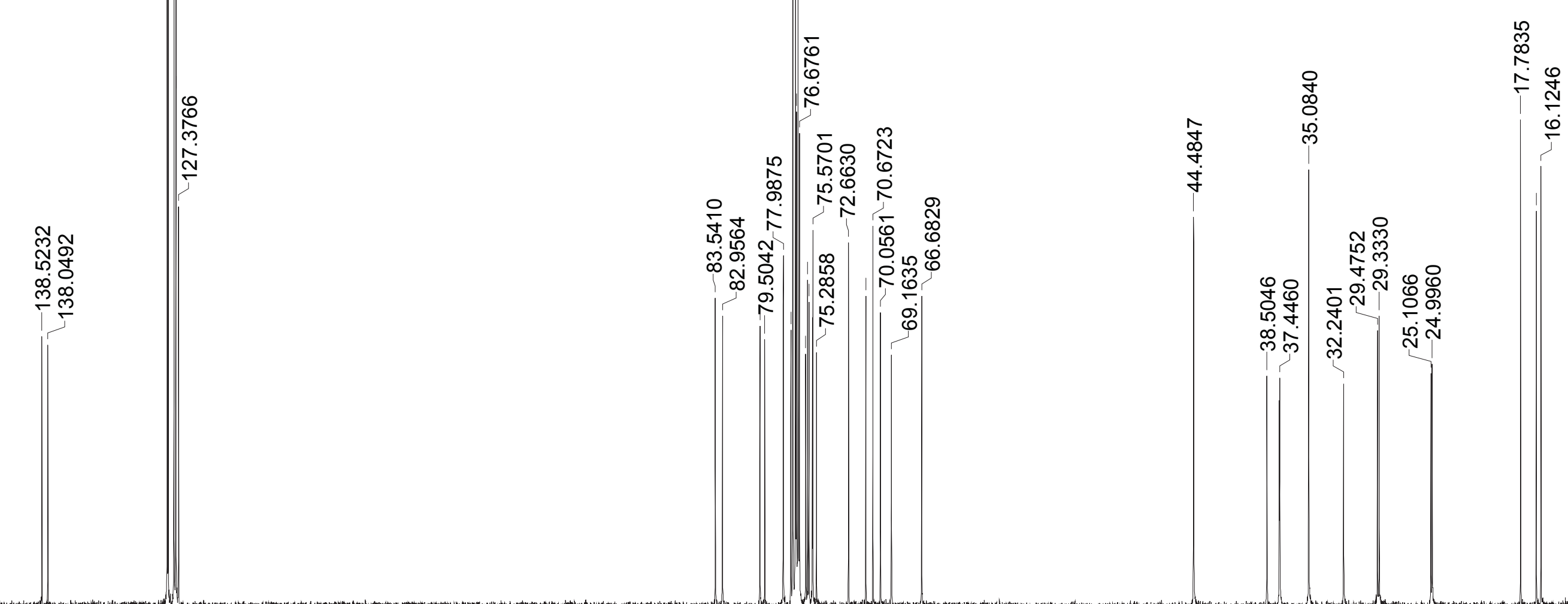


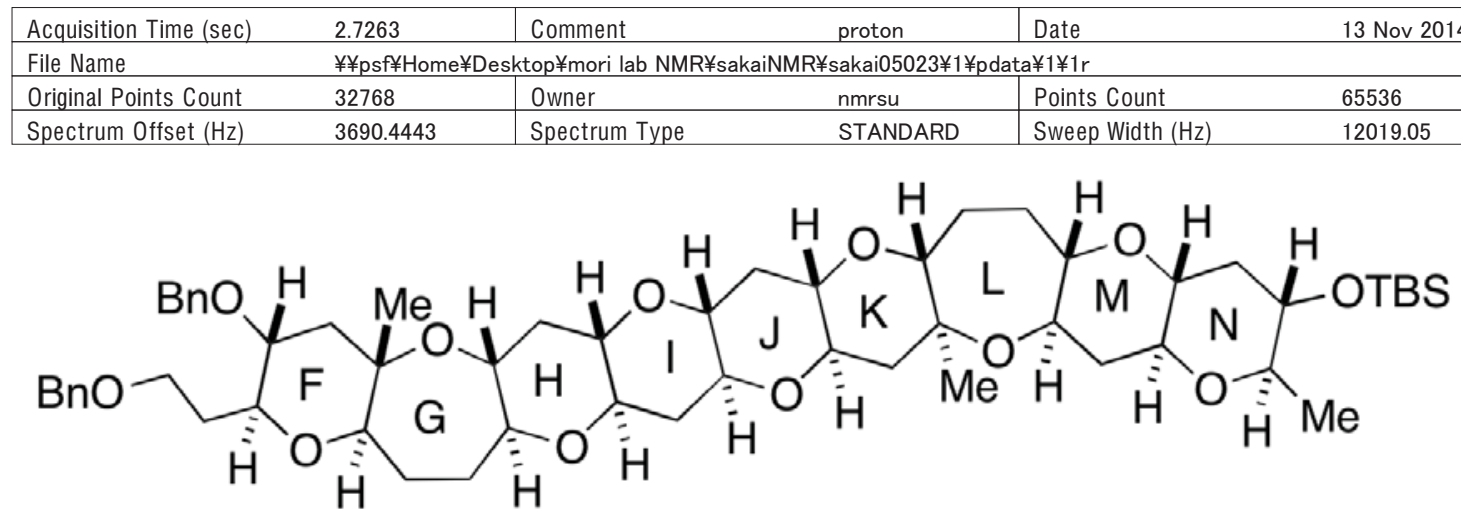

S10

${ }^{1} \mathrm{H} \mathrm{NMR}\left(\mathrm{CDCl}_{3}, 600 \mathrm{MHz}\right)$
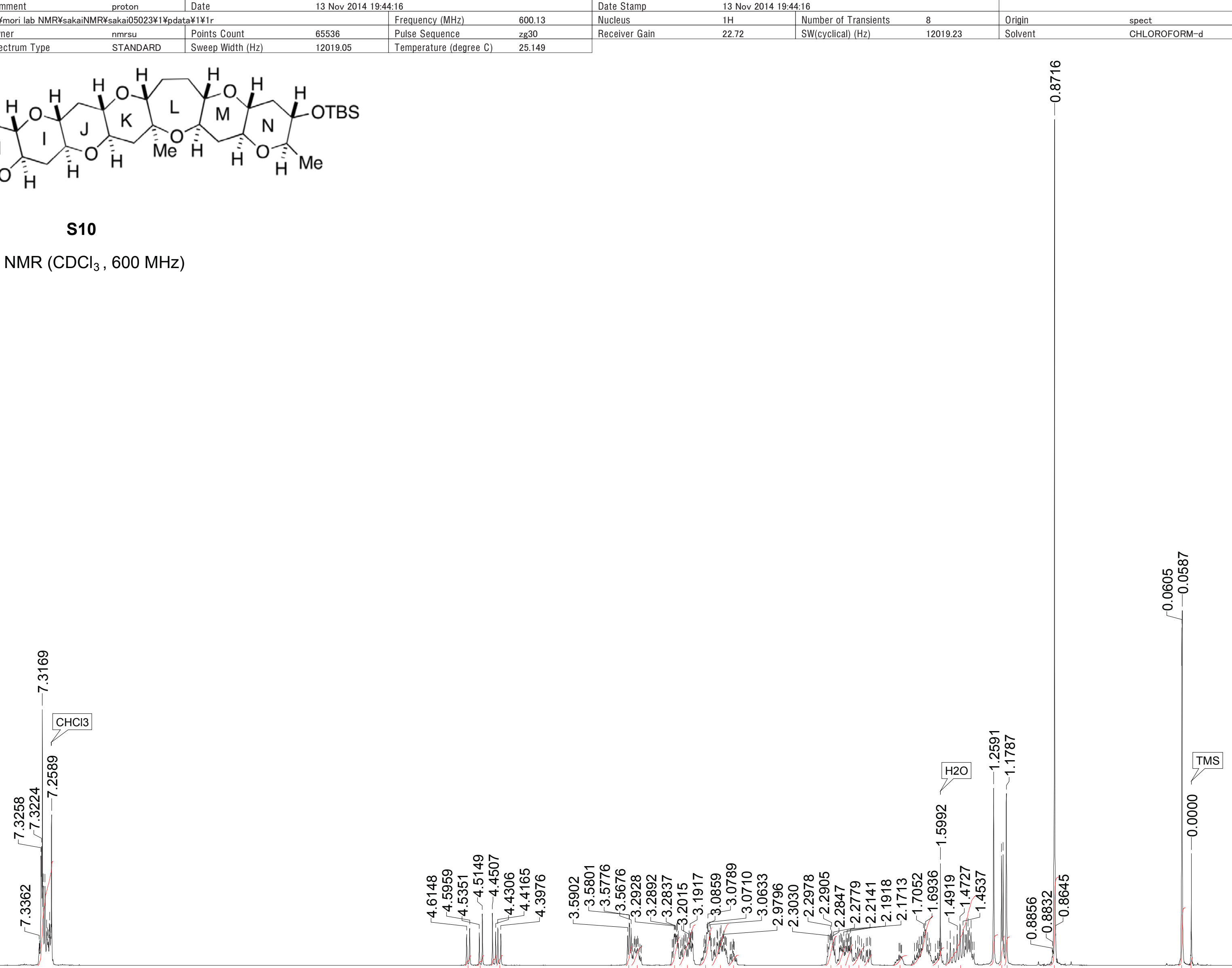

11.01

1.001 .021 .021 .03 2.05 2.03 3.00 4.27 4.014.36 1.08 3.08 2.042.18 2.001.12 1.03 5.201.87 7.31 3.253.10 3.029.36 


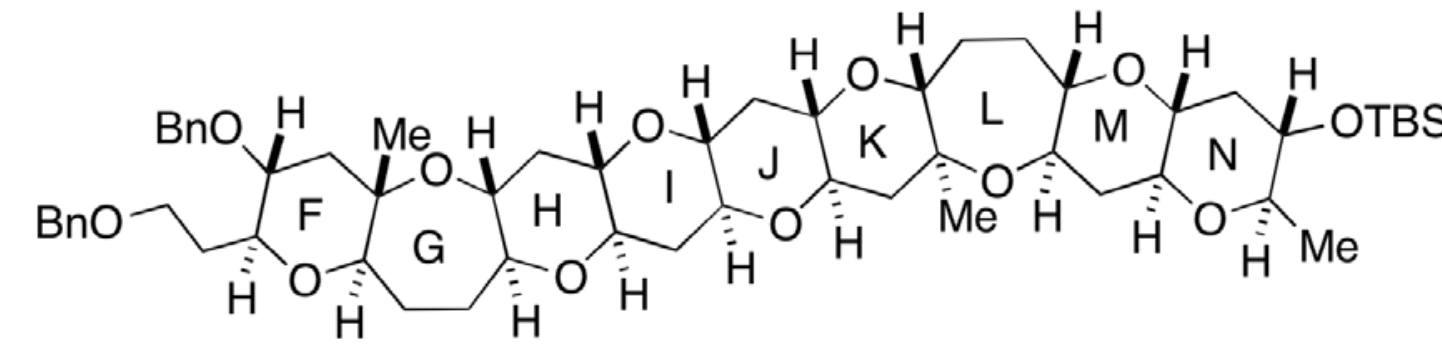

S10

${ }^{13} \mathrm{C} \mathrm{NMR}\left(\mathrm{CDCl}_{3}, 150 \mathrm{MHz}\right)$

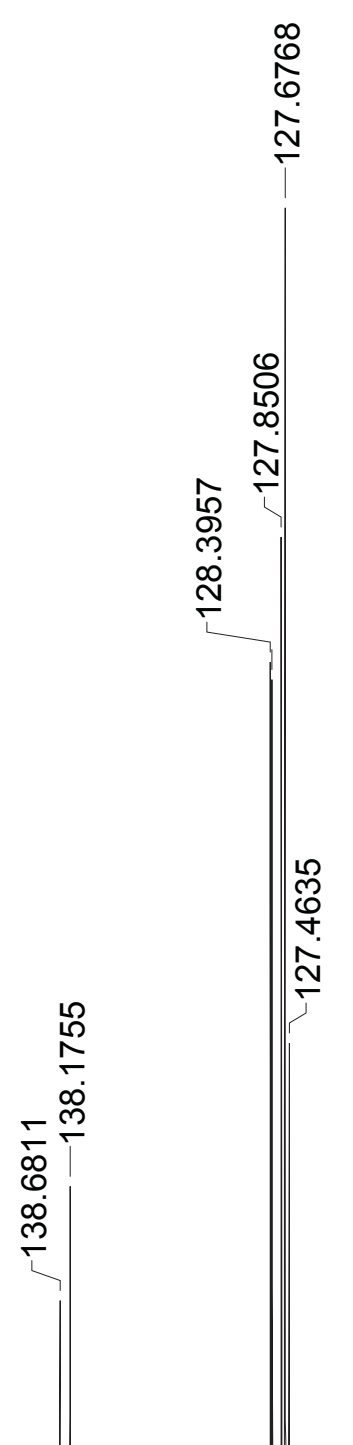

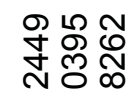

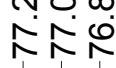

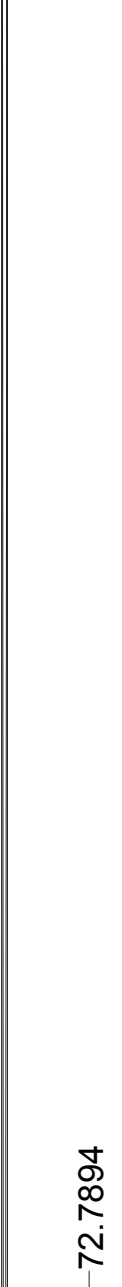

ì
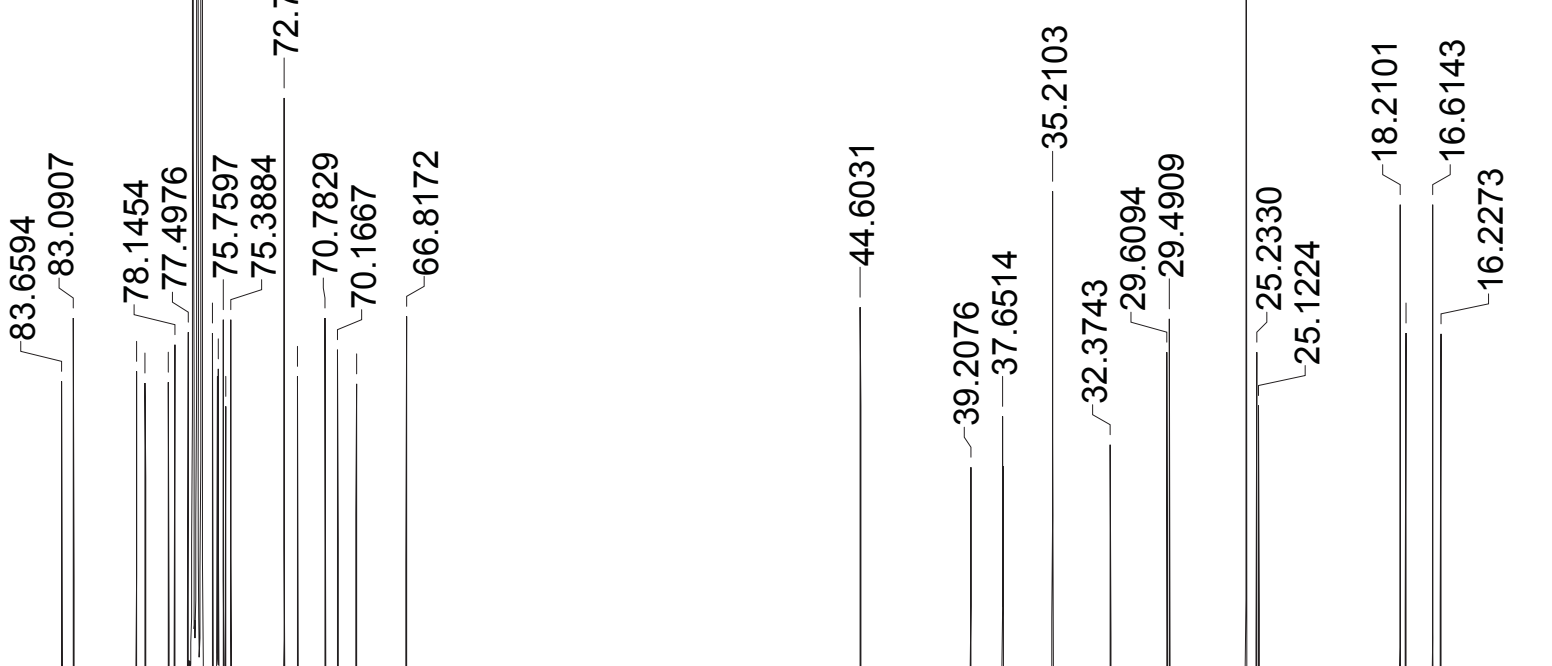


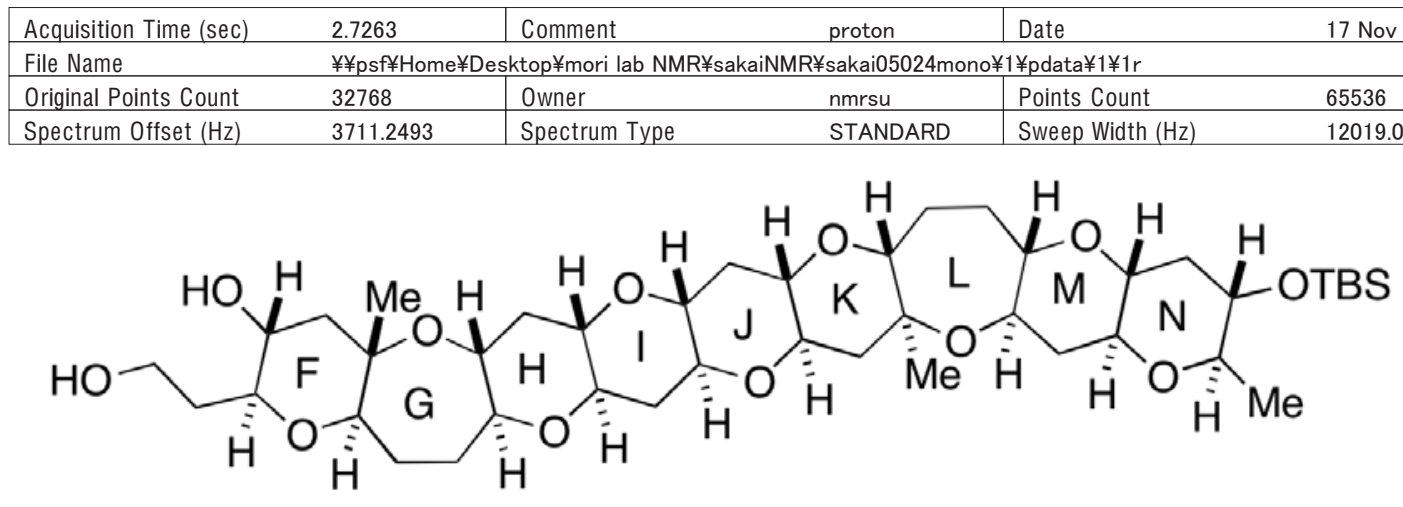

20

${ }^{1} \mathrm{H}$ NMR (pyridine-d5 , $600 \mathrm{MHz}$ )

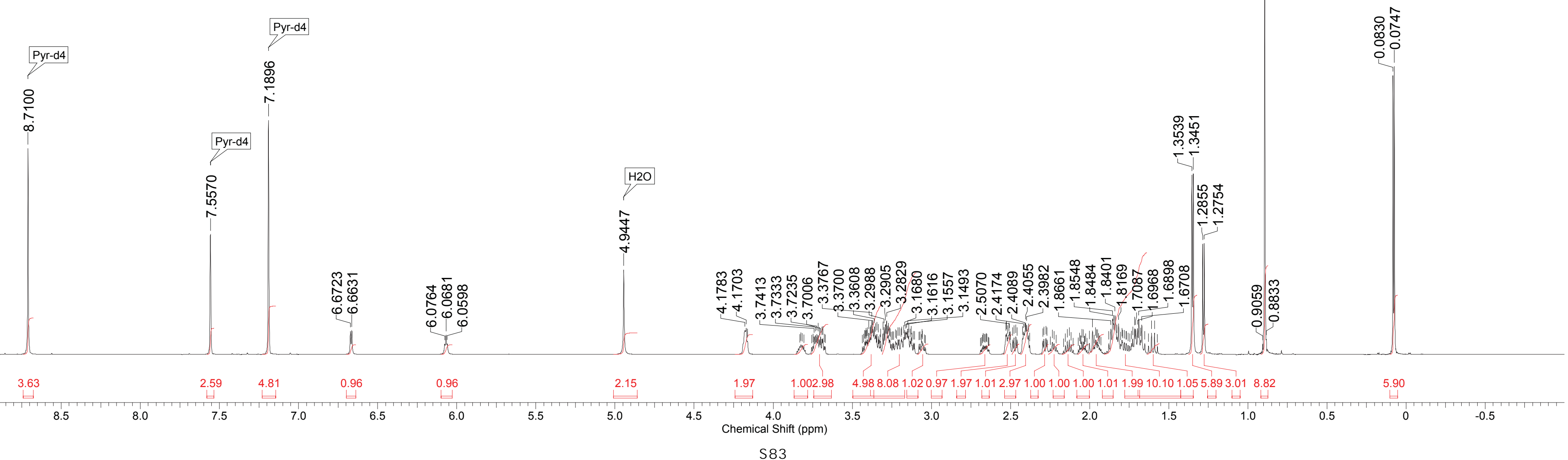


This report was created by ACD/NMR Processor Academic Edition. For more information go to www.acdlabs.com/nmrproc/

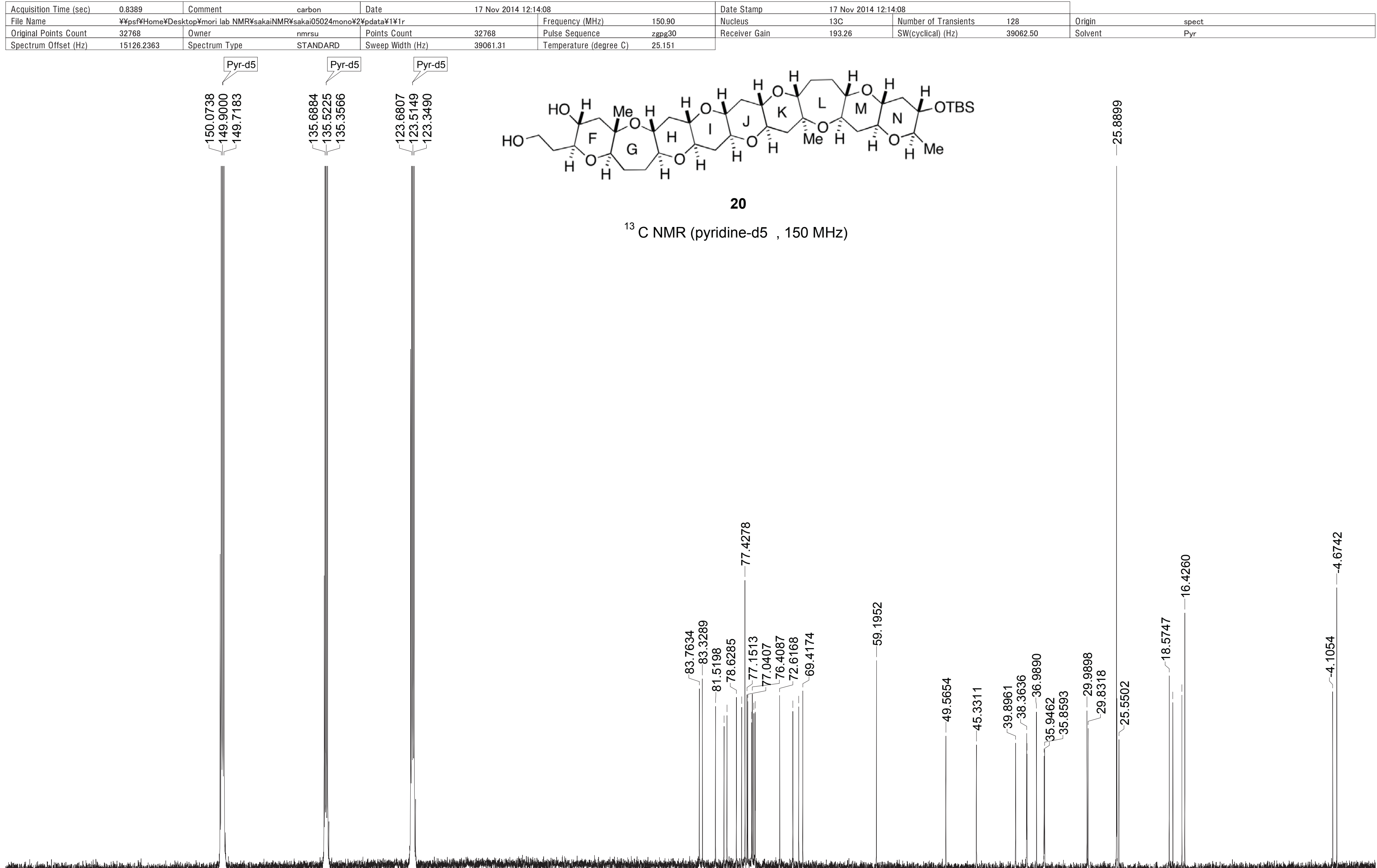




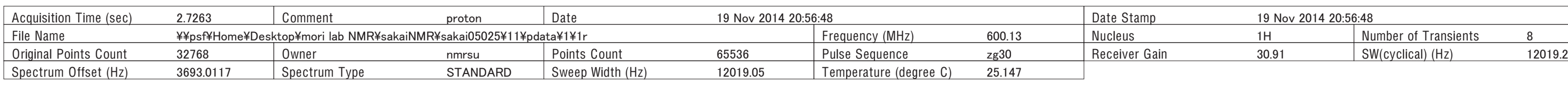

\begin{tabular}{|l} 
Origin \\
Solvent
\end{tabular}

Spectrum Offset (Hz)

S11

${ }^{1} \mathrm{H} \mathrm{NMR}\left(\mathrm{CDCl}_{3}, 600 \mathrm{MHz}\right)$

$\mathrm{CHCl} 3$

$\underset{\substack{0 \\ \stackrel{0}{N}}}{\stackrel{\infty}{\Gamma}}$

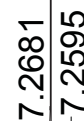

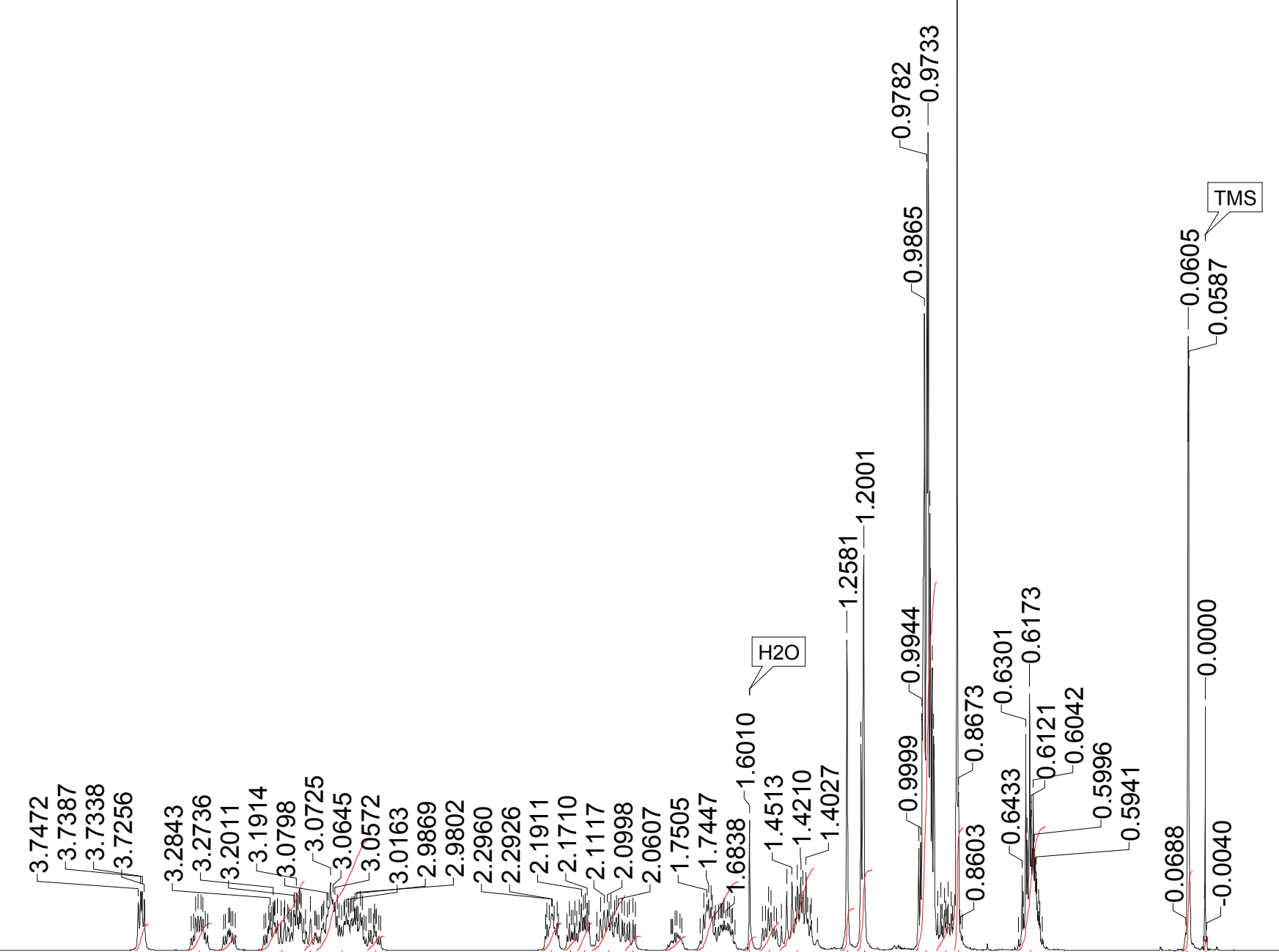

1.10

1.922 .001 .005 .081 .068 .071 .042 .0011 .022 .024 .051 .051 .015 .050 .992 .066 .063 .115 .9227 .132 .589 .039 .085 .820 .92 


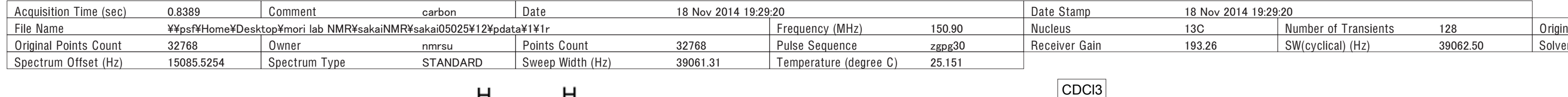

DEIPSO-

\section{S11}

${ }^{13} \mathrm{C} \mathrm{NMR}\left(\mathrm{CDCl}_{3}, 150 \mathrm{MHz}\right)$

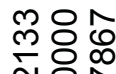

全金
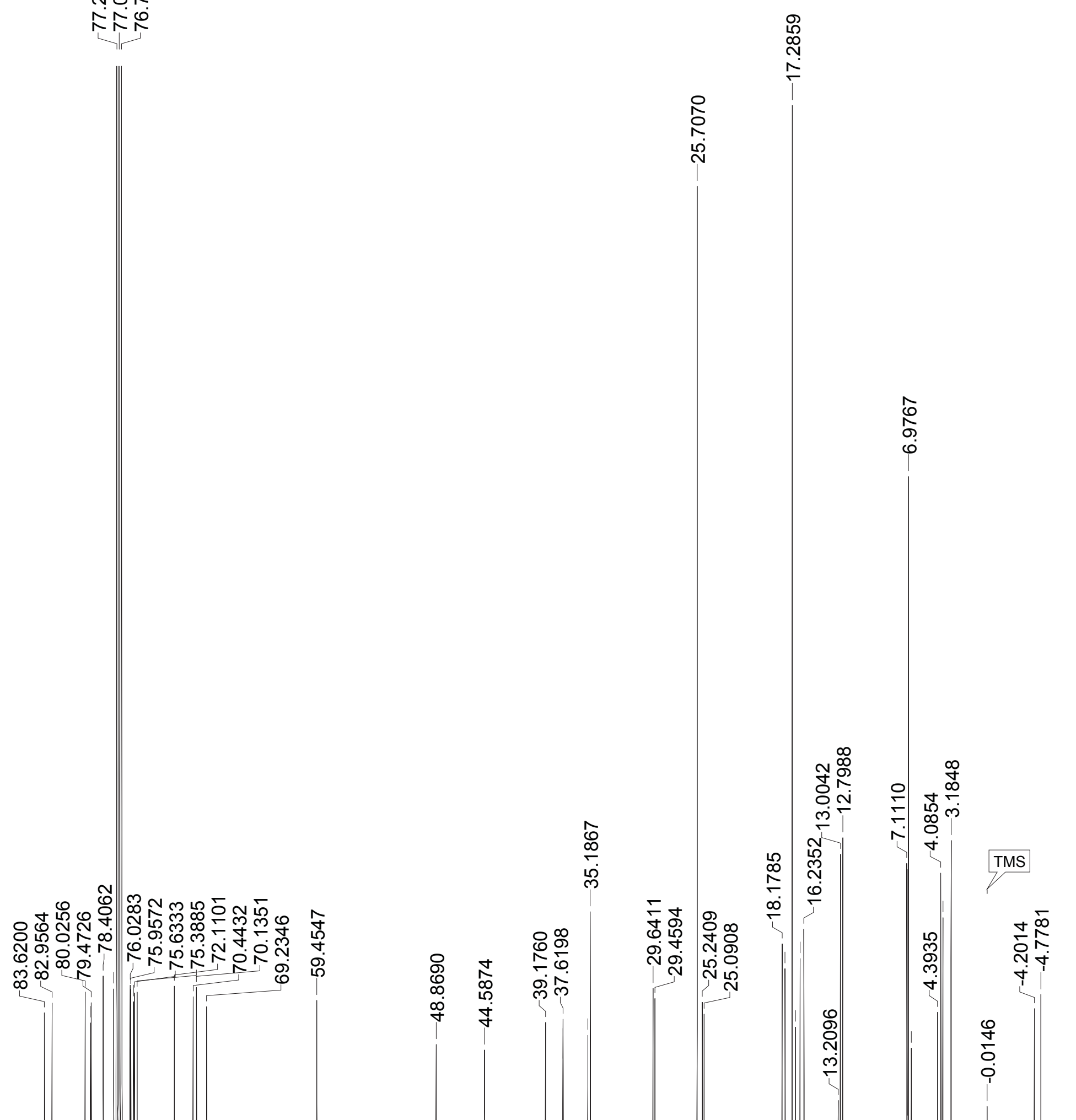
${ }^{1} \mathrm{H}$ NMR $\left(\mathrm{CDCl}_{3}, 600 \mathrm{MHz}\right)$

$\mathrm{CHCl} 3$

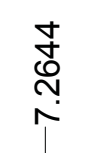

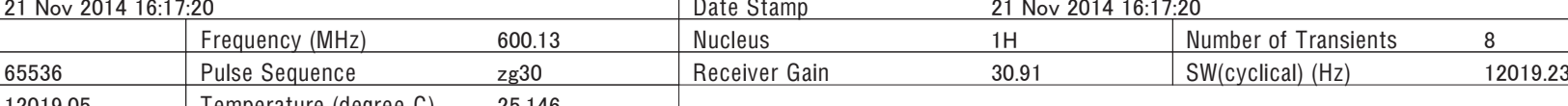
Number of Transients
SW(cyclical) (Hz)

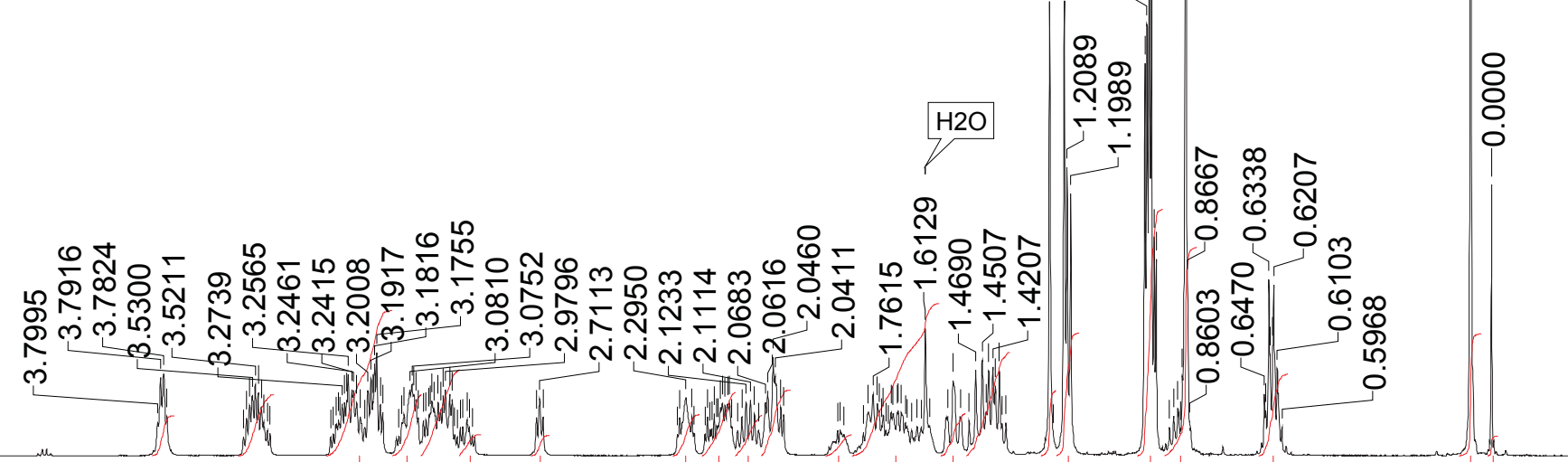

$\begin{array}{lllll}1.99 & 3.03 & 7.183 .024 .261 .041 .00 & 2.033 .131 .993 .251 .037 .532 .085 .10 & 3.226 .0812 .2010 .314 .04\end{array}$ 


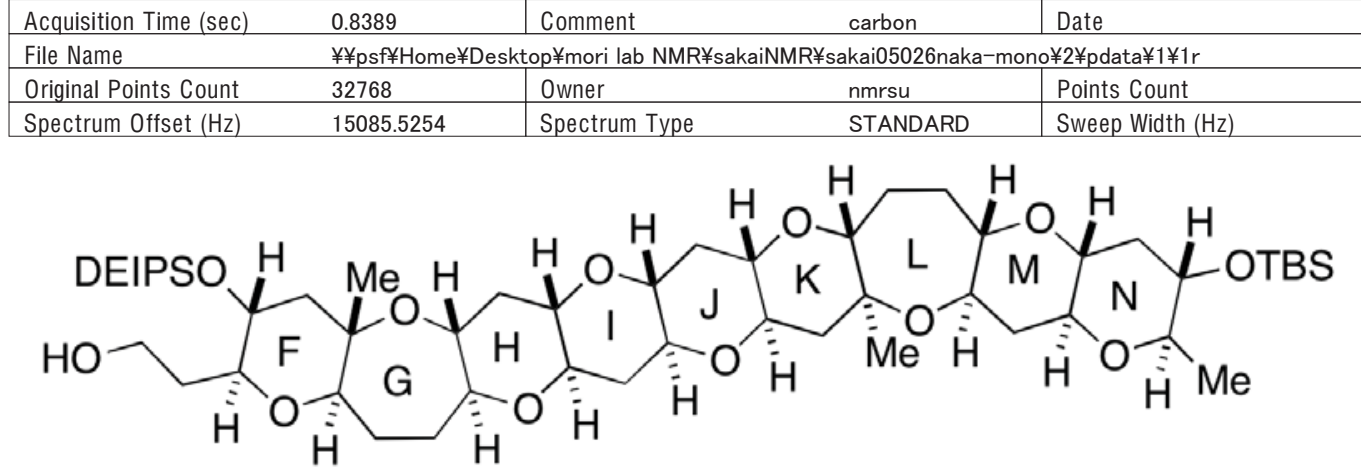

$\mathrm{S} 12$

${ }^{13} \mathrm{C} \mathrm{NMR}\left(\mathrm{CDCl}_{3}, 150 \mathrm{MHz}\right)$

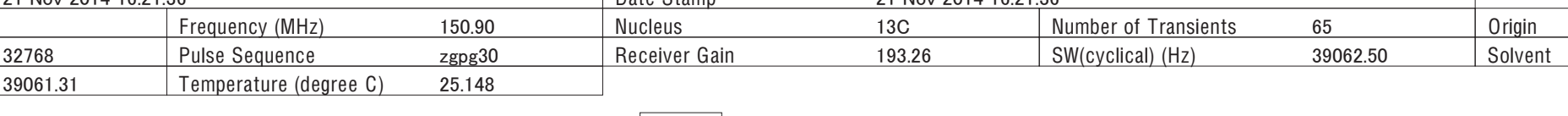

$\mathrm{CDCl3}$

i্co

Níg

is Spect 


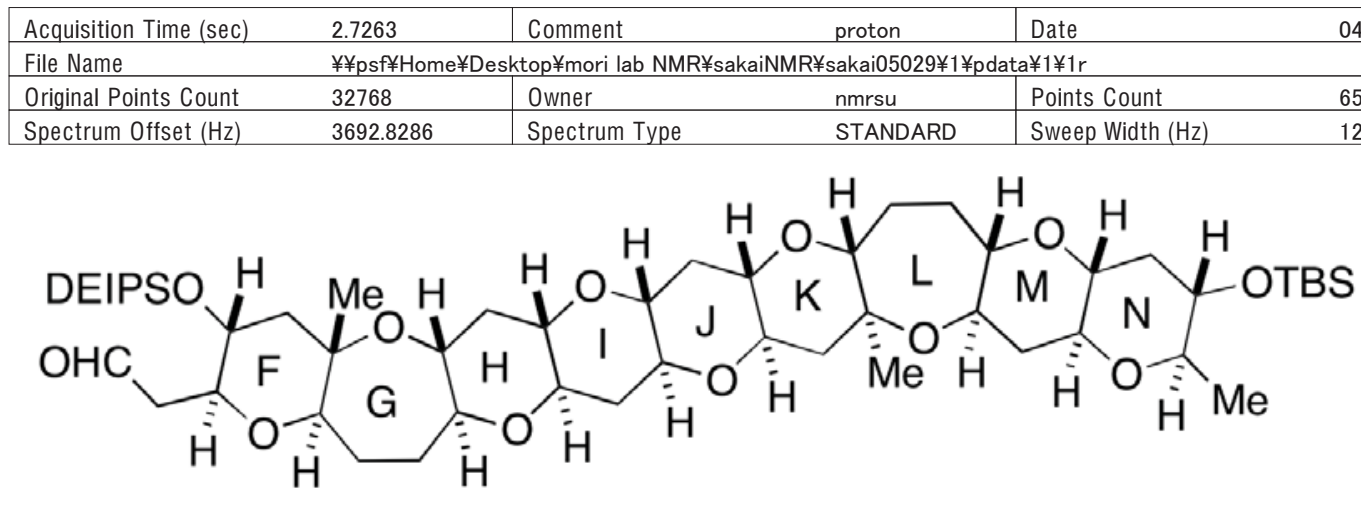

${ }^{1} \mathrm{H} \mathrm{NMR}\left(\mathrm{CDCl}_{3}, 600 \mathrm{MHz}\right)$

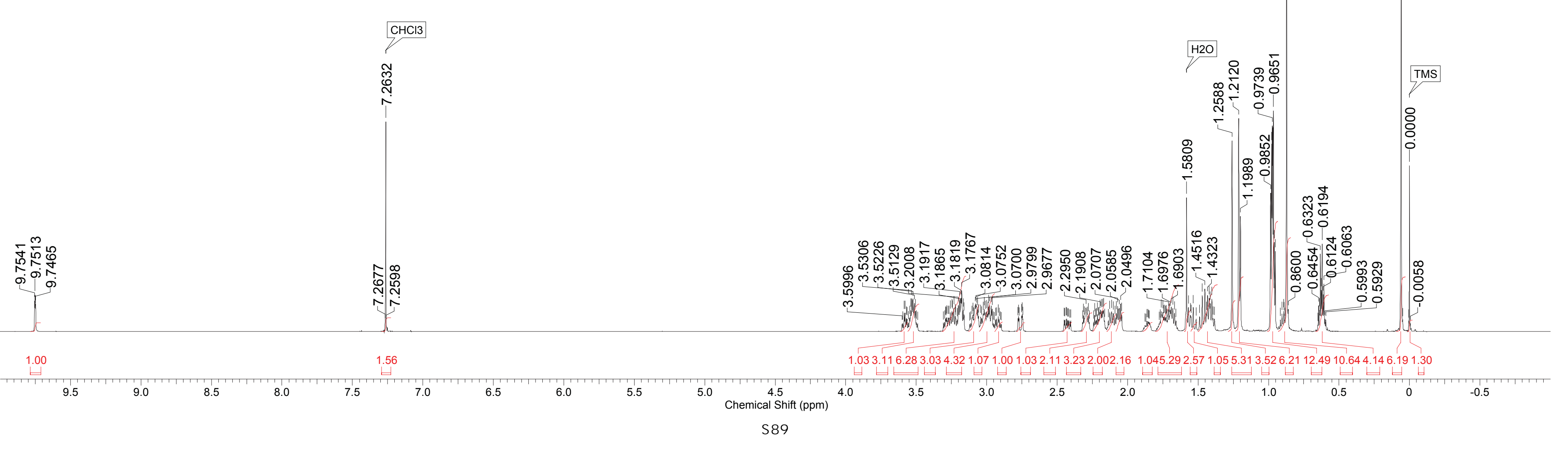




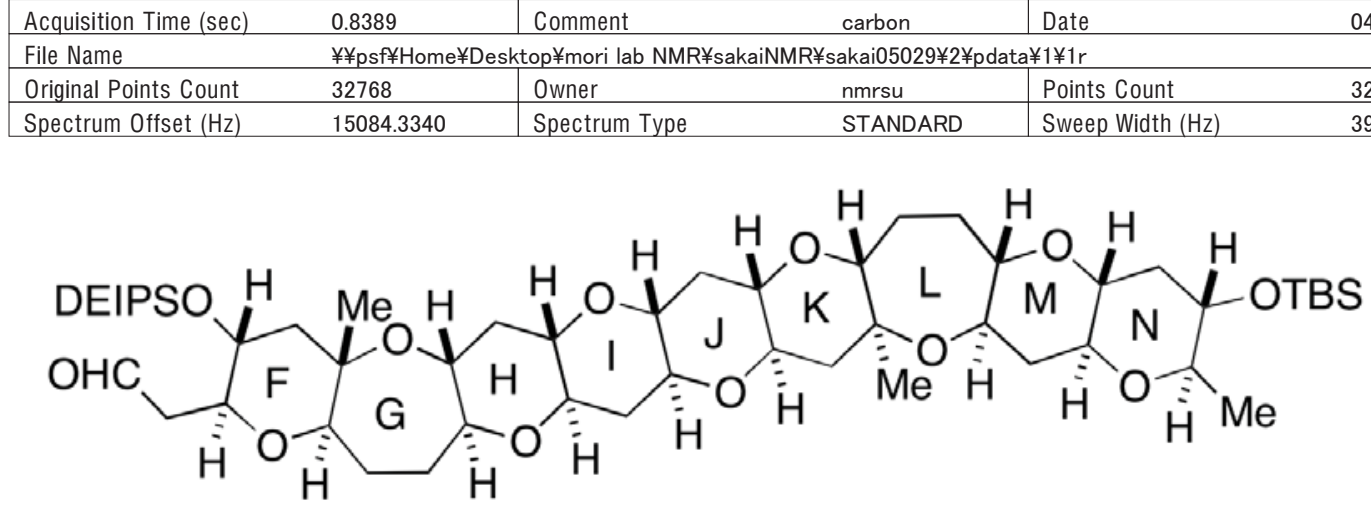

21

${ }^{13} \mathrm{C} \mathrm{NMR}\left(\mathrm{CDCl}_{3}, 150 \mathrm{MHz}\right)$

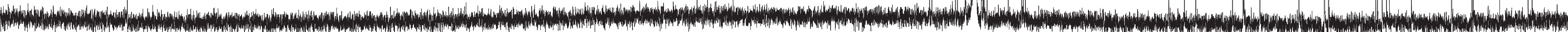

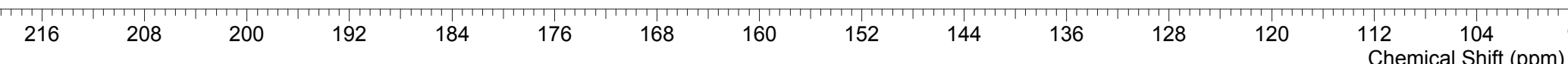

曽 


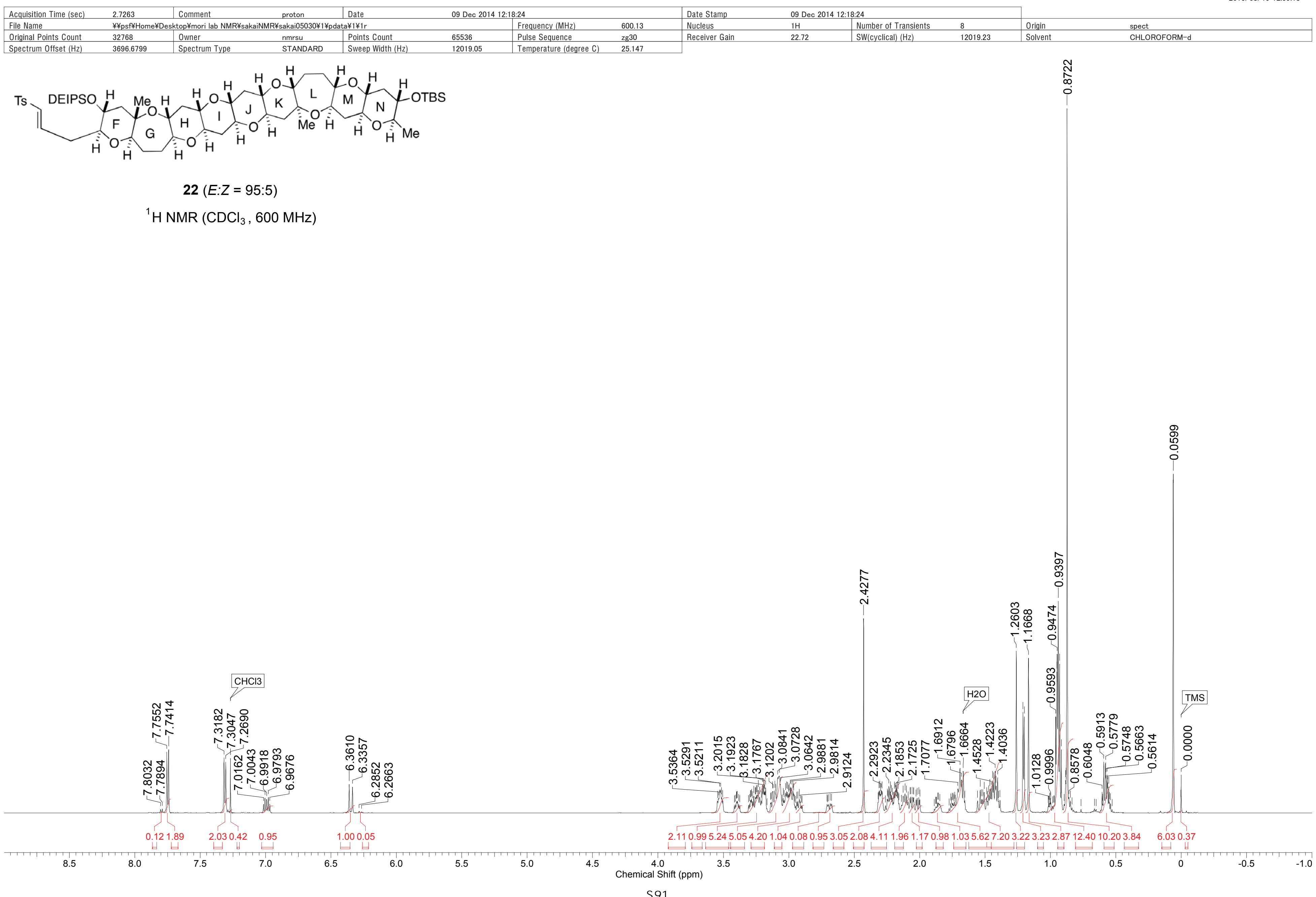






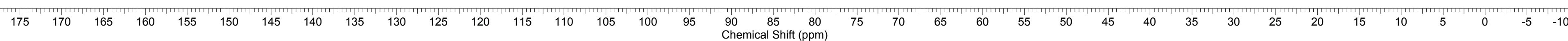




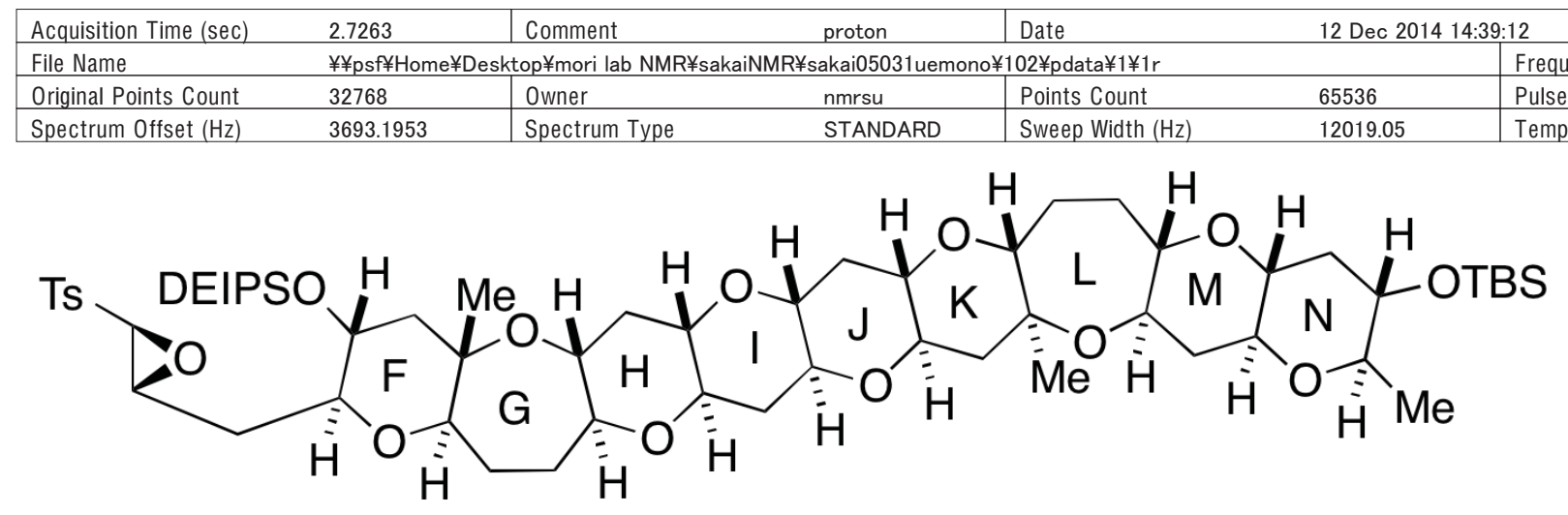

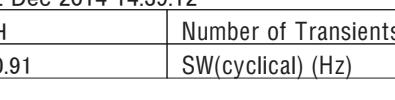

8

Origin

spect

$23(\mathrm{dr}=66: 34)$

${ }^{1} \mathrm{H} \mathrm{NMR}\left(\mathrm{CDCl}_{3}, 600 \mathrm{MHz}\right)$

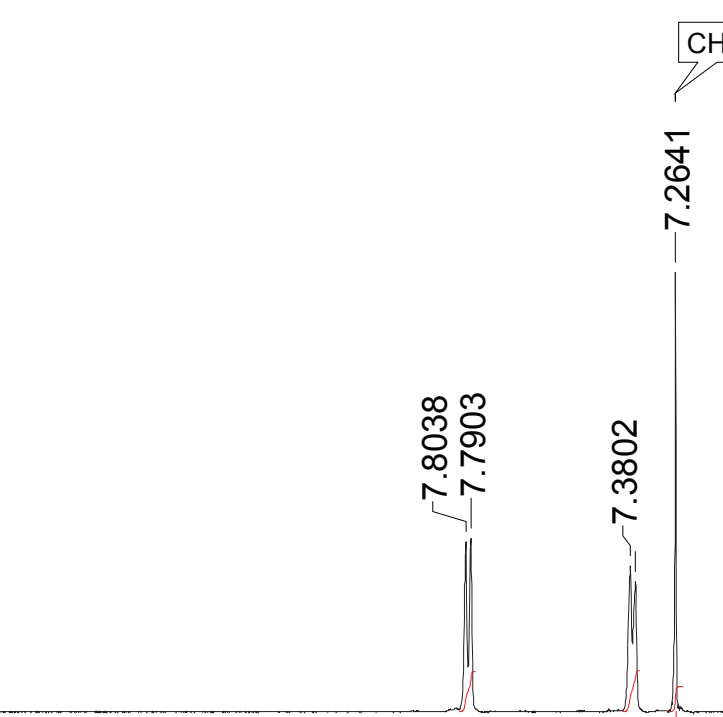

$\mathrm{CHCl3}$

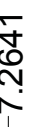

$1.95 \quad 1.981 .22$

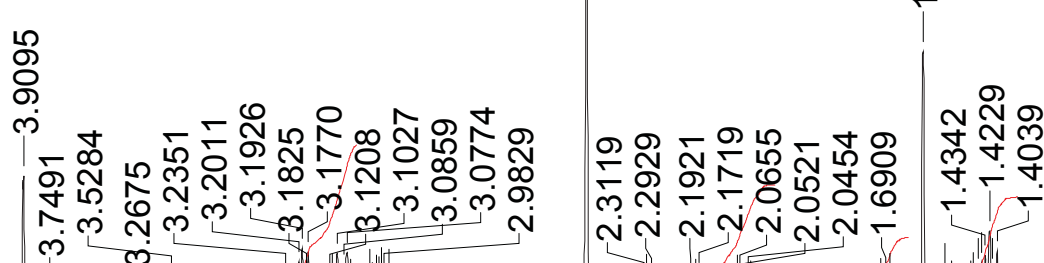

if ${ }^{2}$.

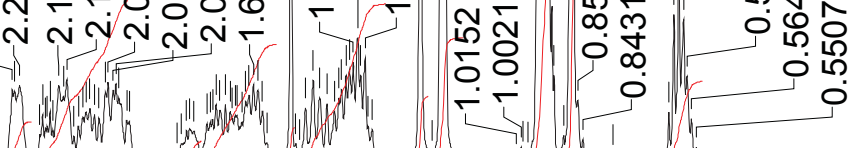




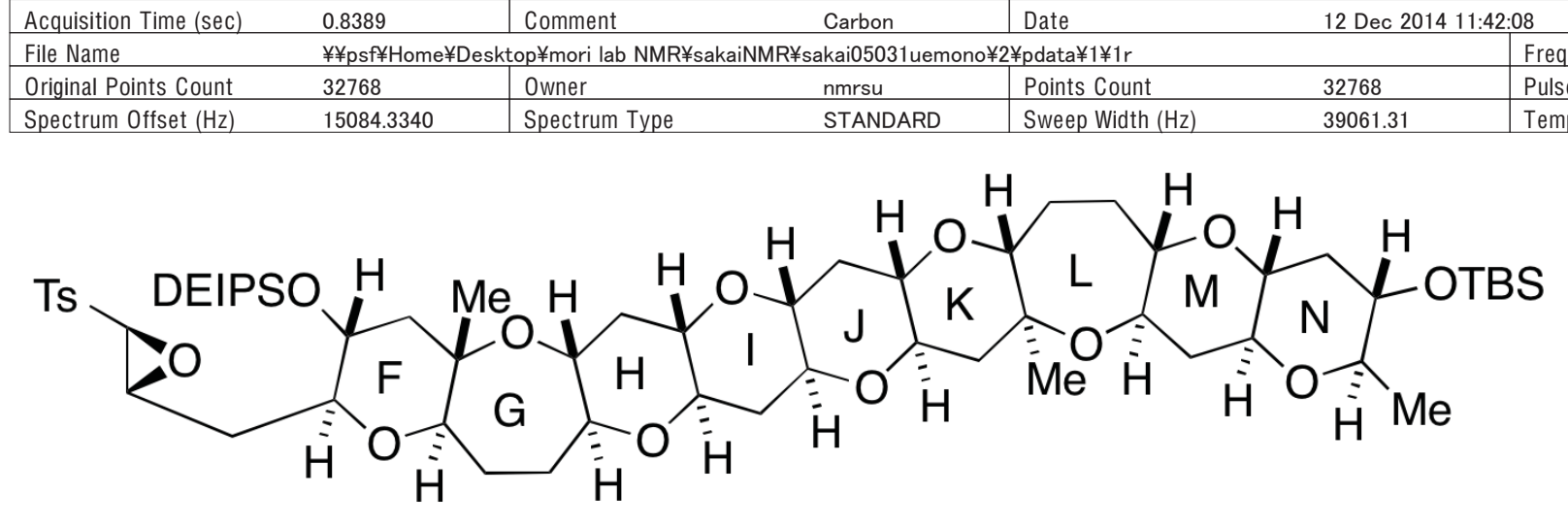

$23(\mathrm{dr}=66: 34)$

${ }^{13} \mathrm{C} \mathrm{NMR}\left(\mathrm{CDCl}_{3}, 150 \mathrm{MHz}\right)$
$\mathrm{CDCl3}$
Origin
Solvent

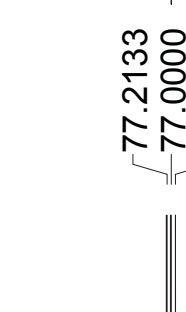

CNMR (CDCl, $150 \mathrm{MHz})$

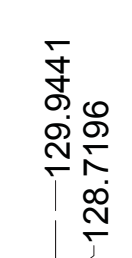

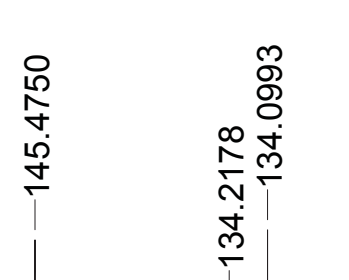

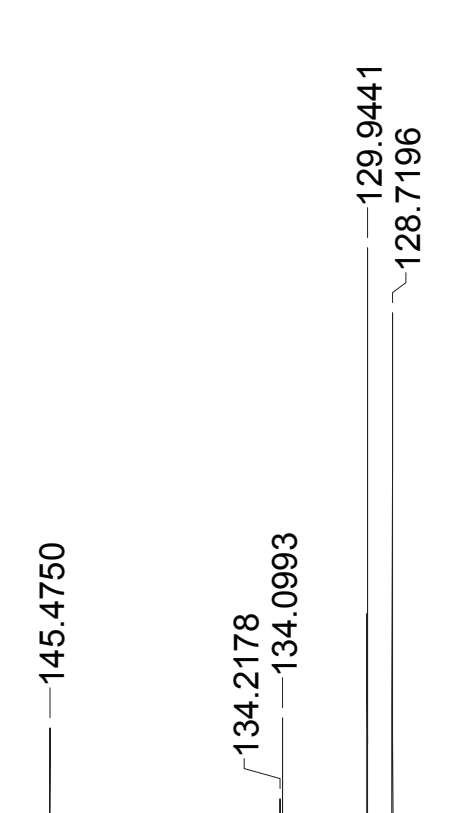

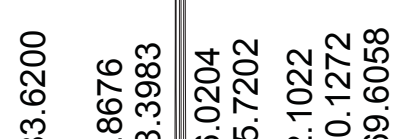

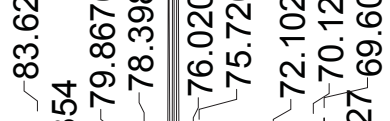



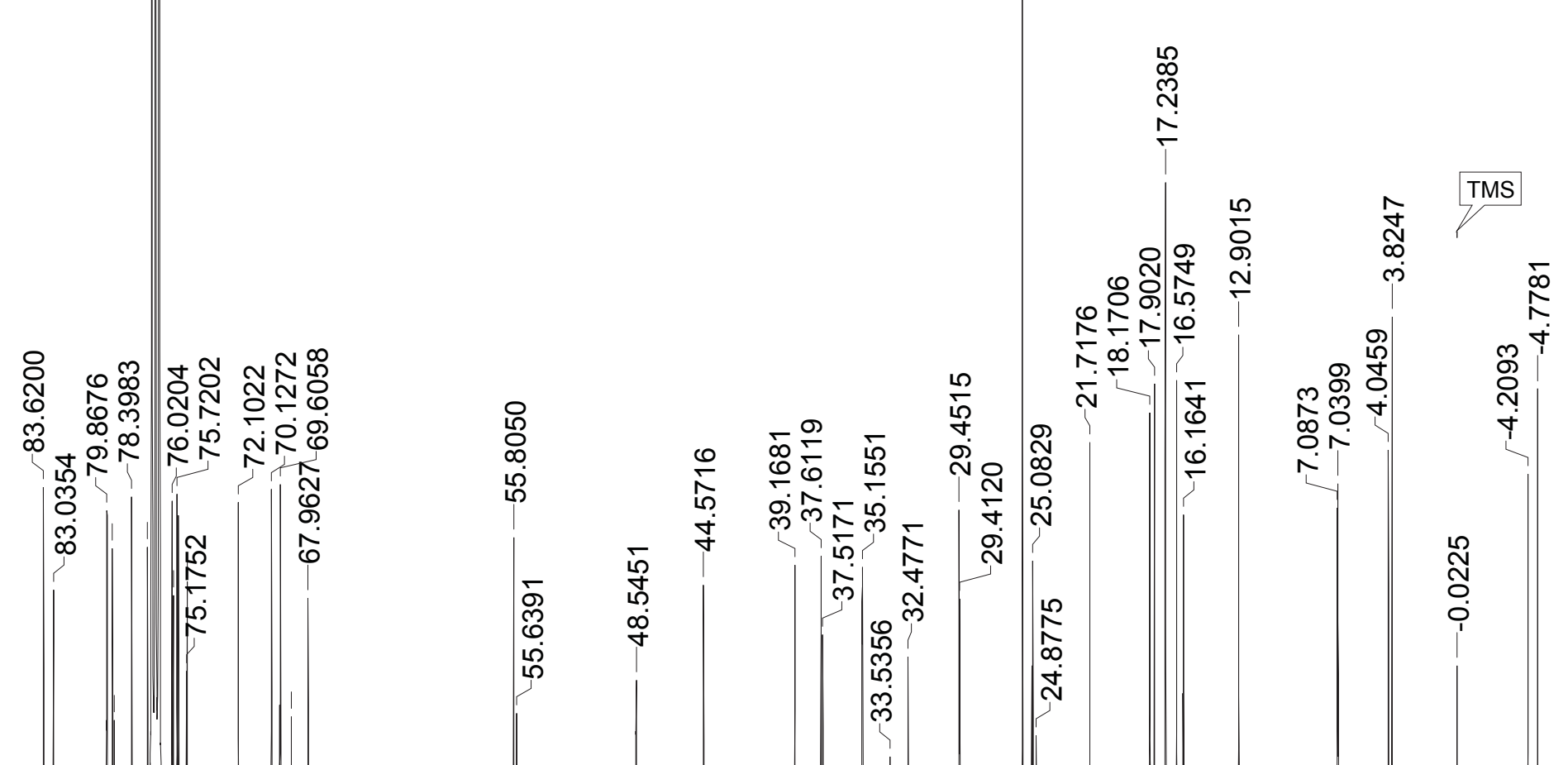

Whowats

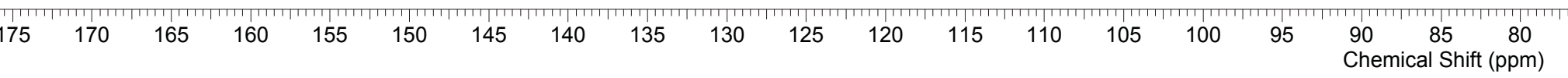




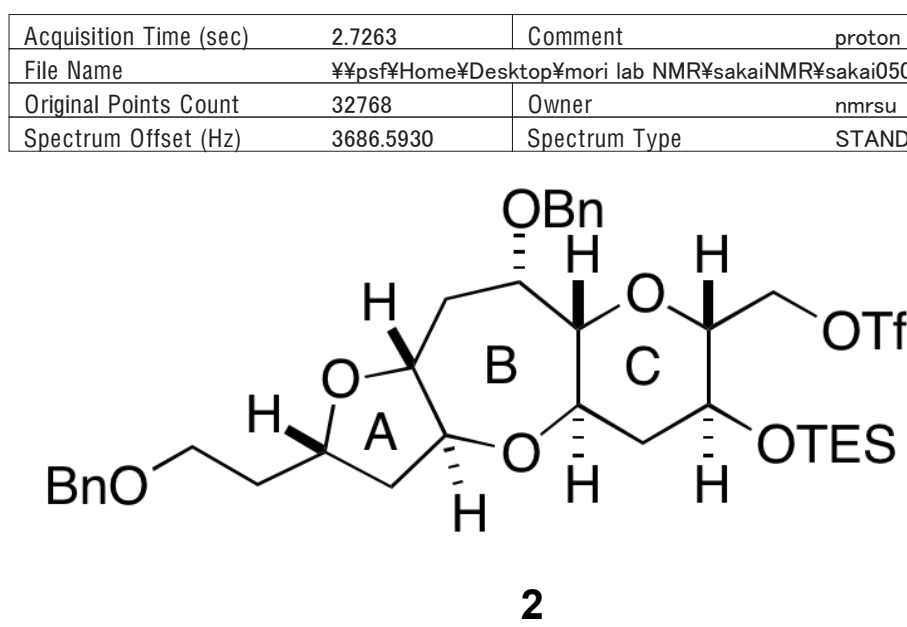

\begin{tabular}{l|ll|l} 
& Frequency (MHz) & 600.13 & Nucleus \\
\hline 65536 & Pulse Sequence & 2830 & Receiver Gain \\
\hline
\end{tabular}

\begin{tabular}{ll} 
Number of Transients & 8 \\
\hline SW(cyclical) (Hz) & 1201923
\end{tabular}

Origin

spect

${ }^{1} \mathrm{H} \mathrm{NMR}\left(\mathrm{CDCl}_{3}, 600 \mathrm{MHz}\right)$

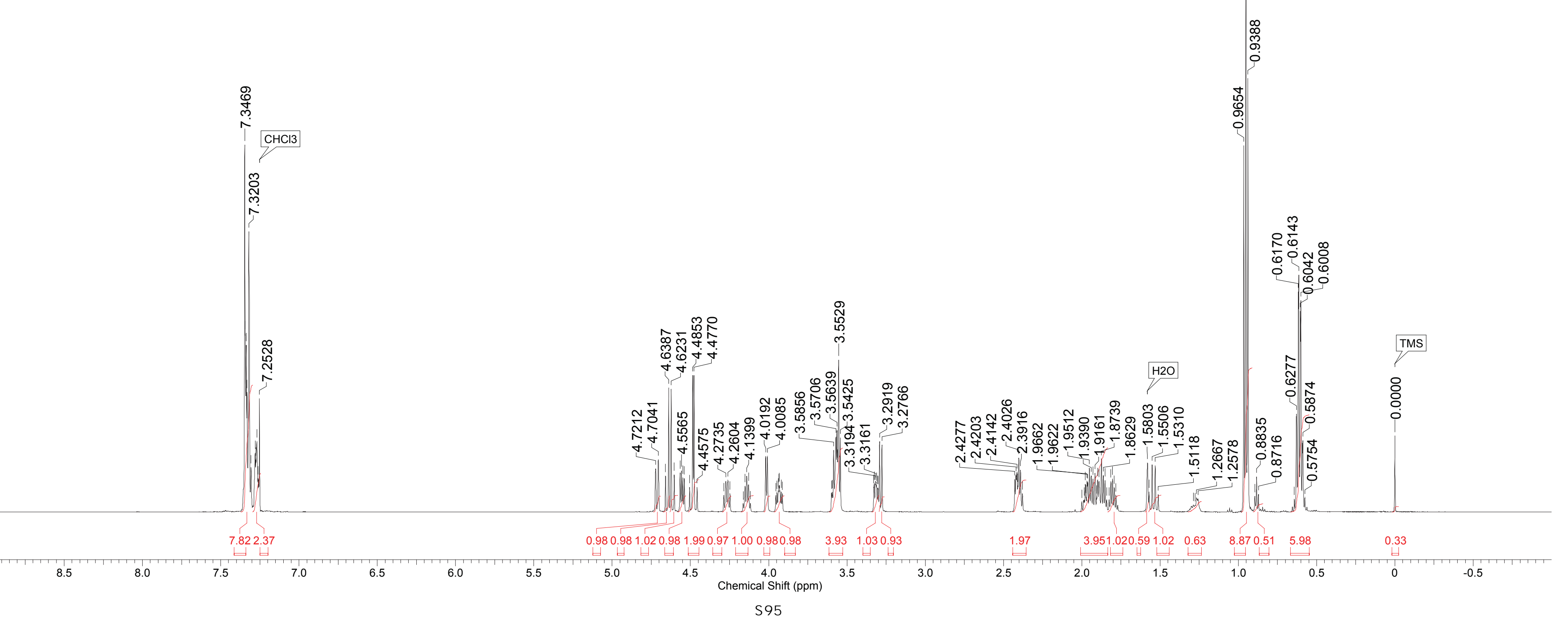


This report was created by ACD/NMR Processor Academic Edition. For more information go to www.acdlabs.com/nmrproc/

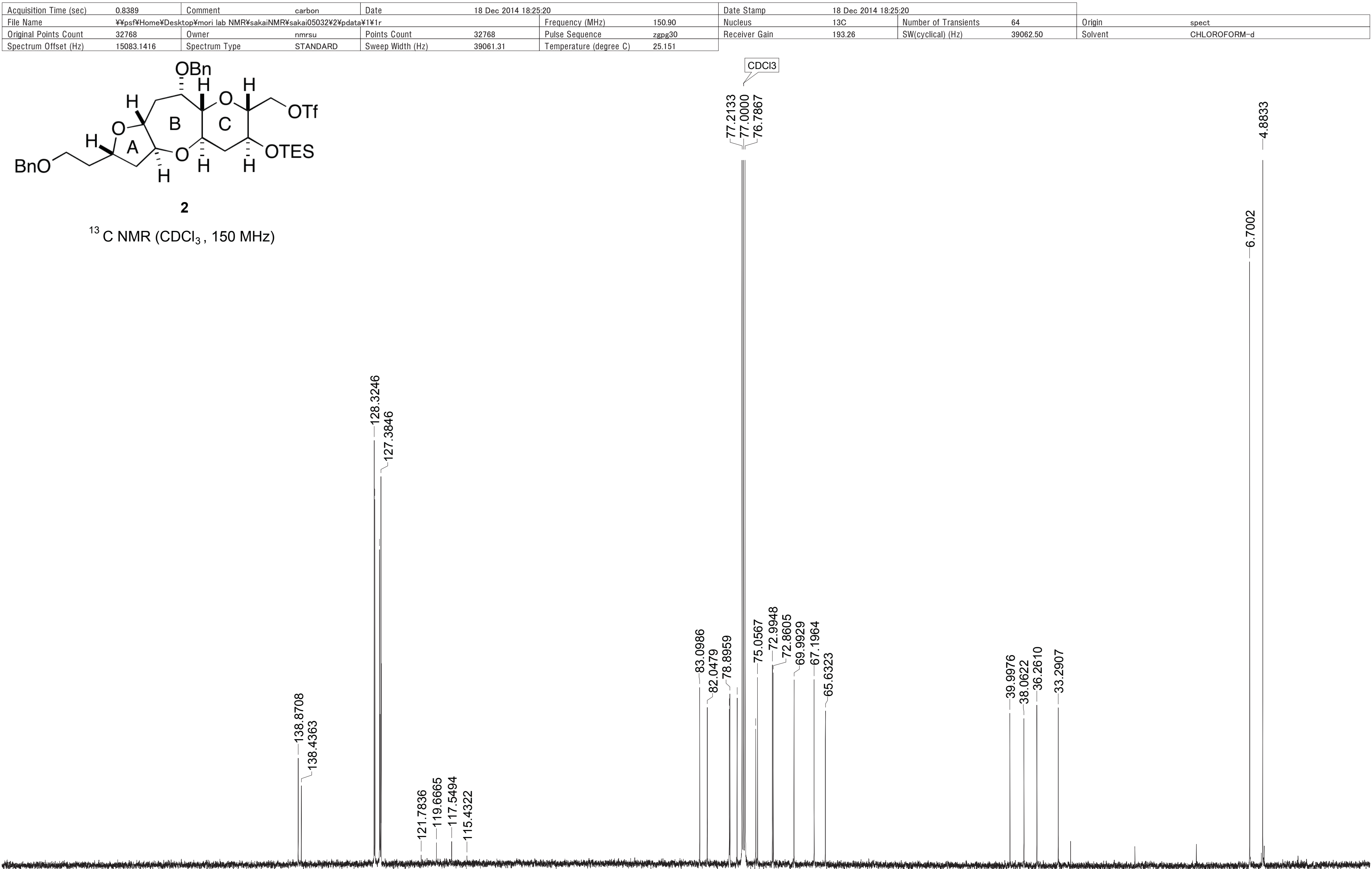




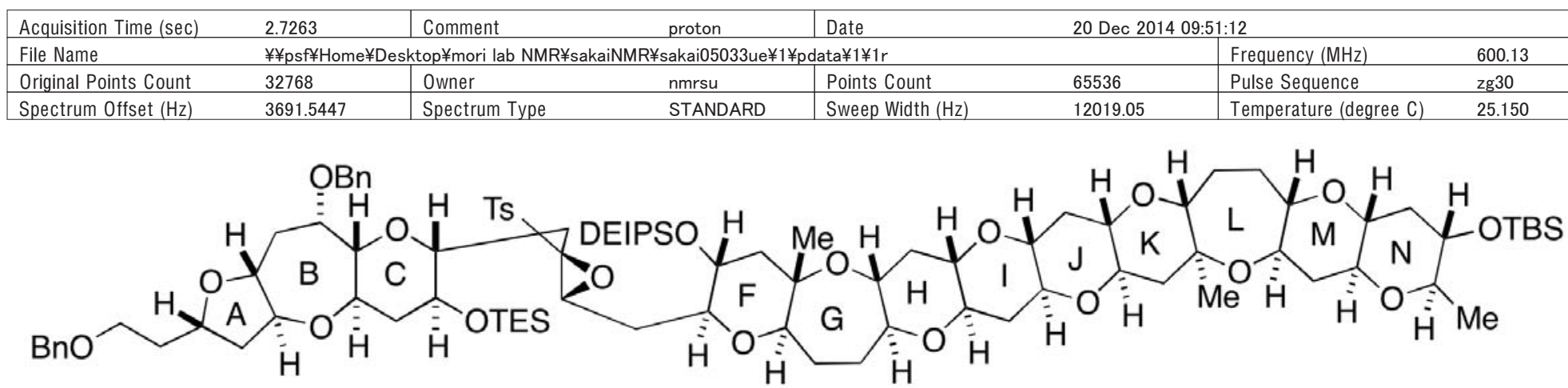

24 (major isomer)

${ }^{1} \mathrm{H}$ NMR $\left(\mathrm{CDCl}_{3}, 600 \mathrm{MHz}\right)$
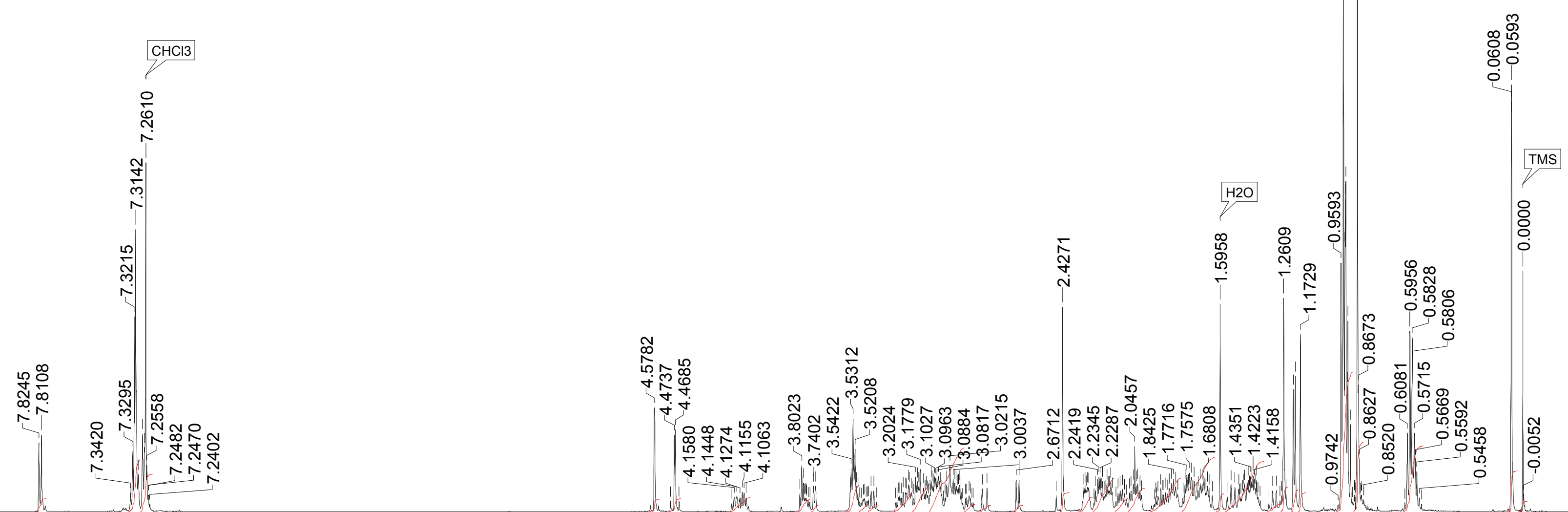

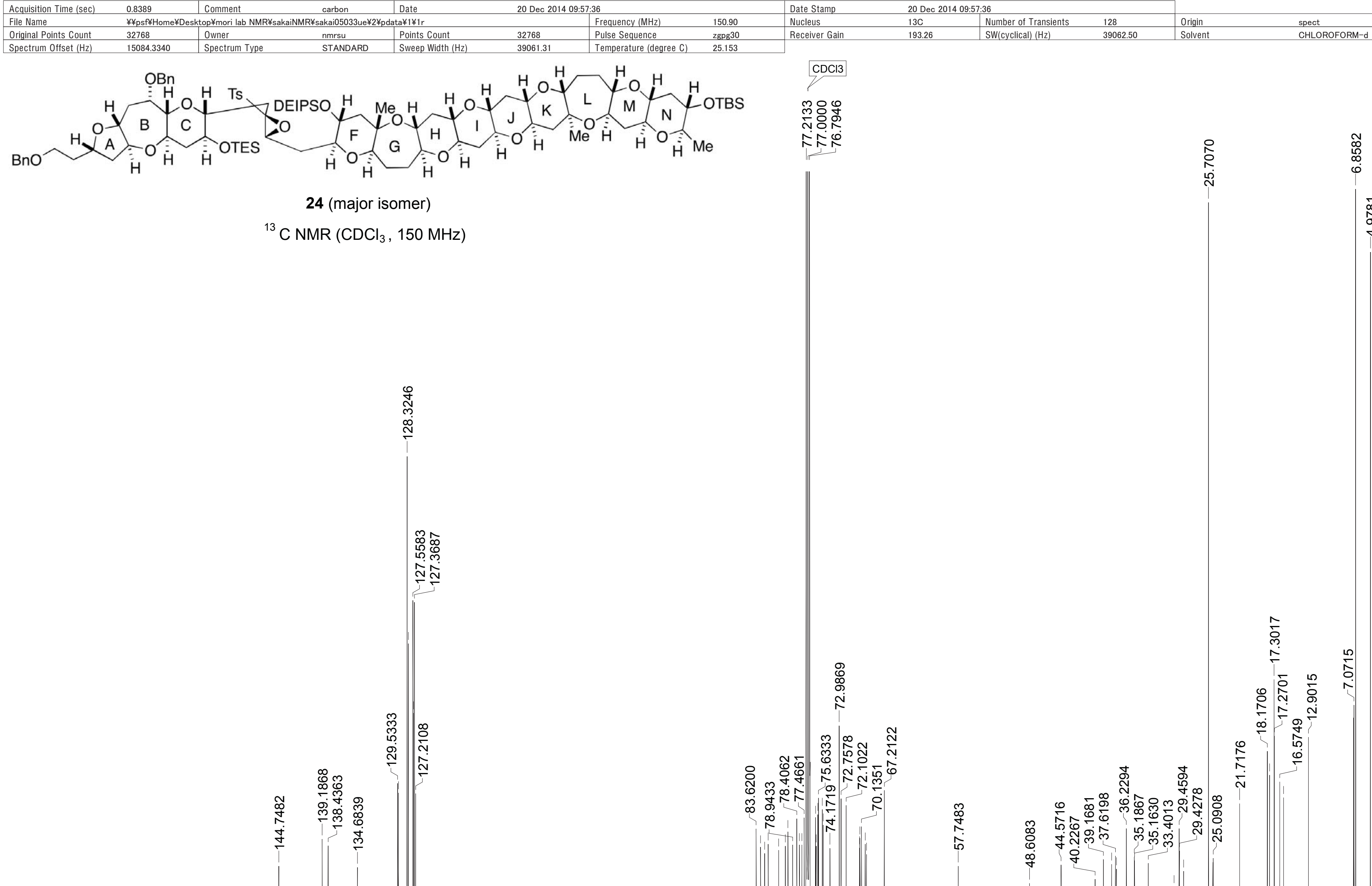


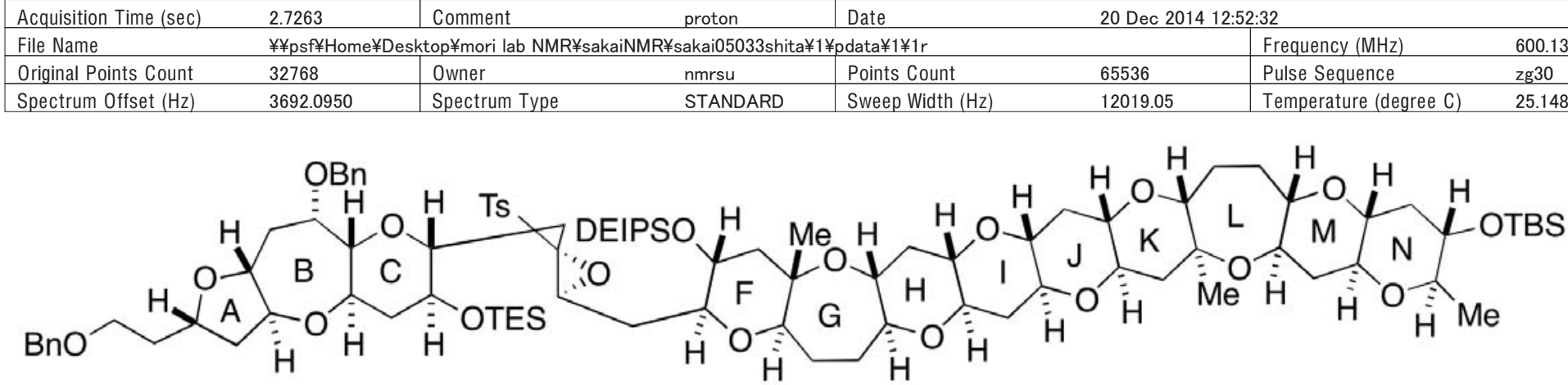

24 (minor isomer)

${ }^{1} \mathrm{H} \mathrm{NMR}\left(\mathrm{CDCl}_{3}, 600 \mathrm{MHz}\right)$

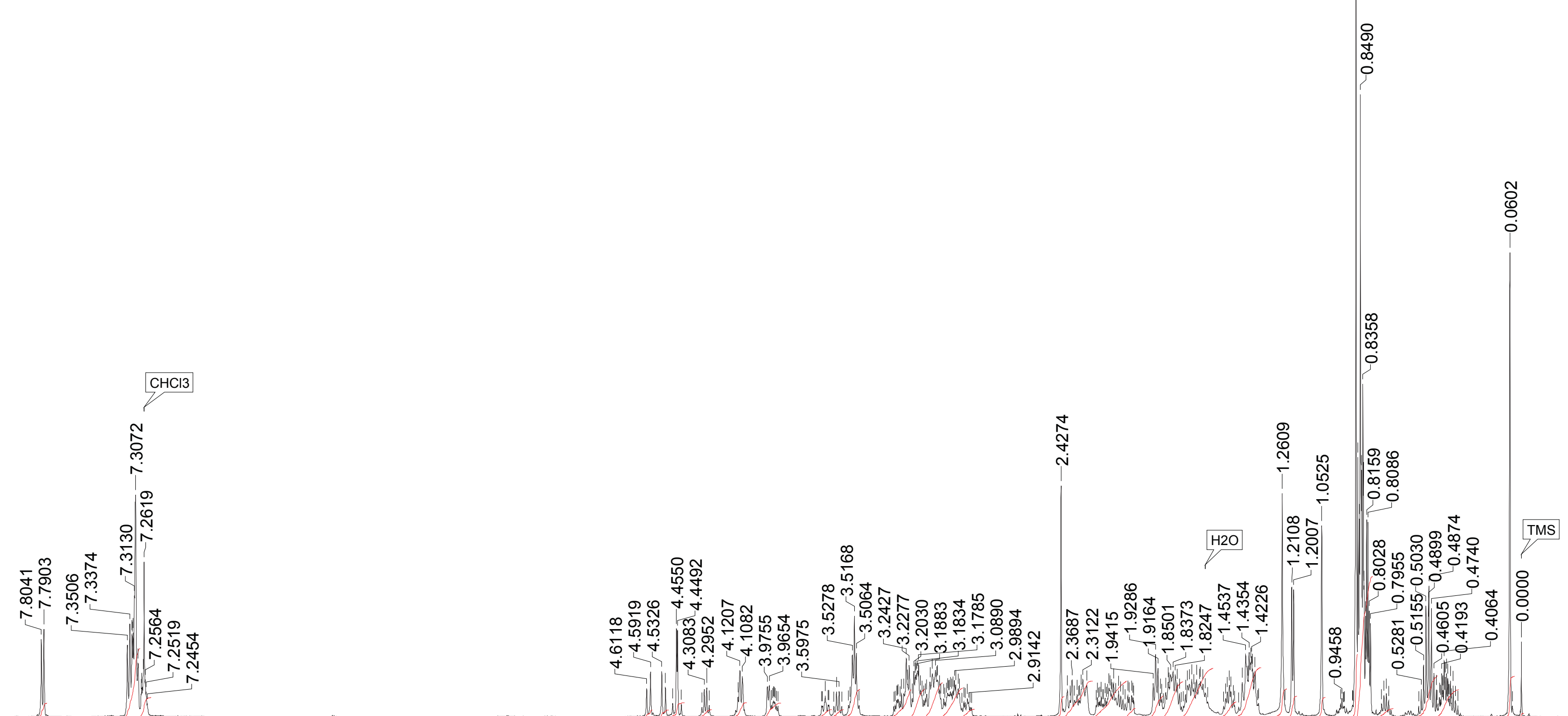

$1.94 \quad 10.042 .78$

0.960 .962 .031 .002 .041 .981 .011 .024 .023 .954 .054 .974 .211 .052 .945 .235 .201 .203 .045 .127 .112 .147 .214 .063 .002 .849 .1920 .621 .165 .993 .975 .910 .46

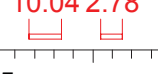

6.5

6.0

5.5

5.0

$4.5 \quad 4.0$
Chemical Shift (ppm) 


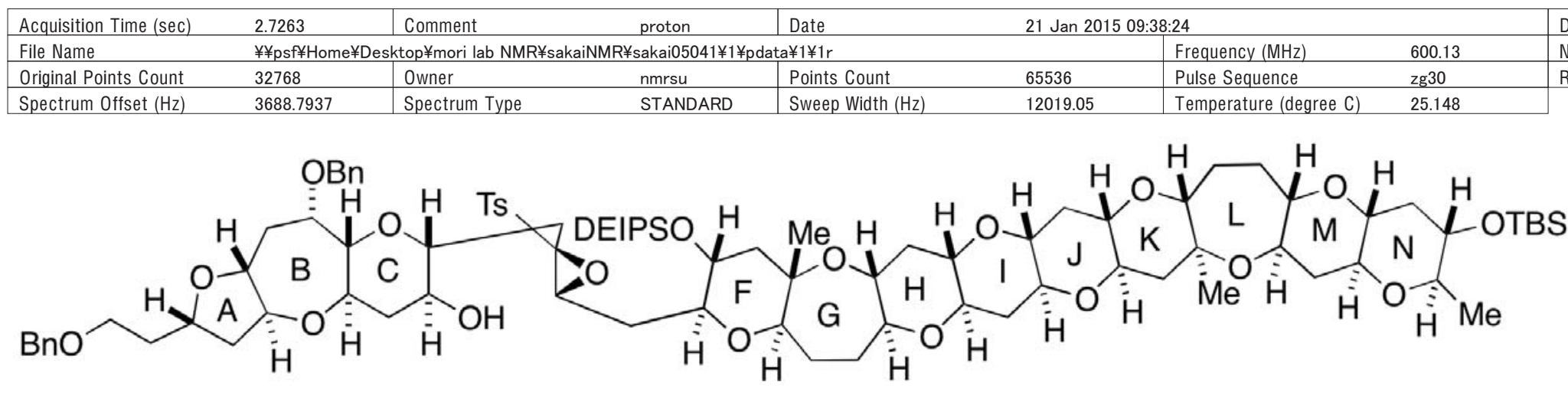

$\mathrm{S} 14(\mathrm{dr}=66: 34)$

${ }^{1} \mathrm{H} \mathrm{NMR}\left(\mathrm{CDCl}_{3}, 600 \mathrm{MHz}\right)$
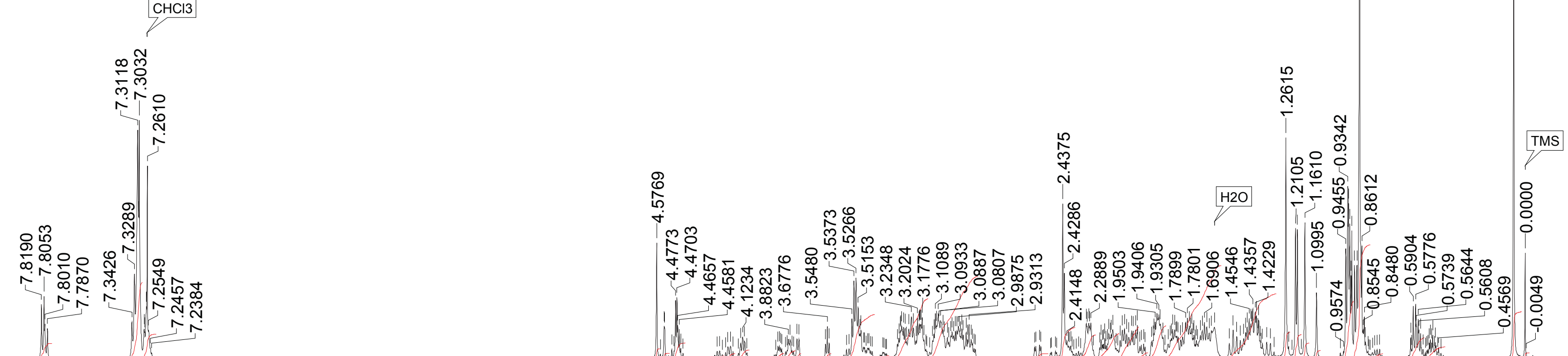

$2.05-10.313 .29$

1.32 0.72 2.11 0.34 0.66 1.14 0.68 1.030.66 0.65 5.930.37 8.1111.10 0.66 0.35 4.22 3.183.394.26 0.784.44 12.59 8.78 3.60 3.25 2.09 1.07 7.77 15.42 0.64 2.81 1.57 6.33 0.77

$8.5 \quad 8.0 \quad 7.5 \quad 7.0$




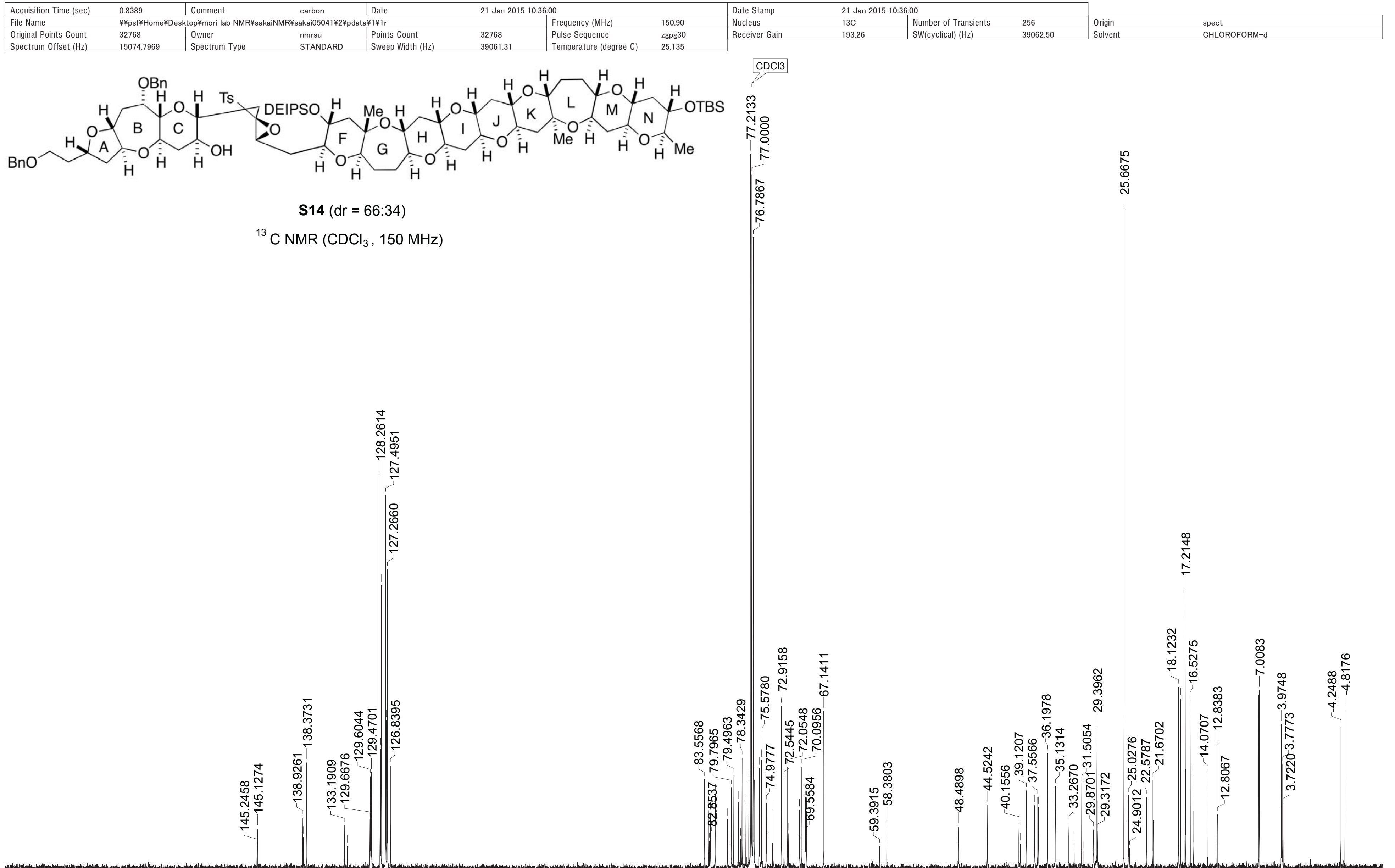



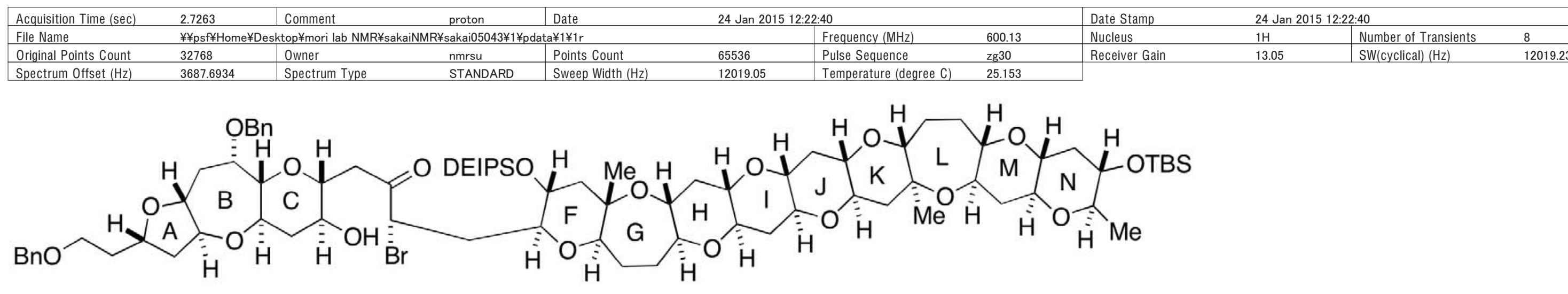

\begin{tabular}{|l|l|}
\hline Origin \\
\hline Solvent
\end{tabular}

spect
CHLOROFORM-d

S15 $(d r=71: 29)$

${ }^{1} \mathrm{H} \mathrm{NMR}\left(\mathrm{CDCl}_{3}, 600 \mathrm{MHz}\right)$

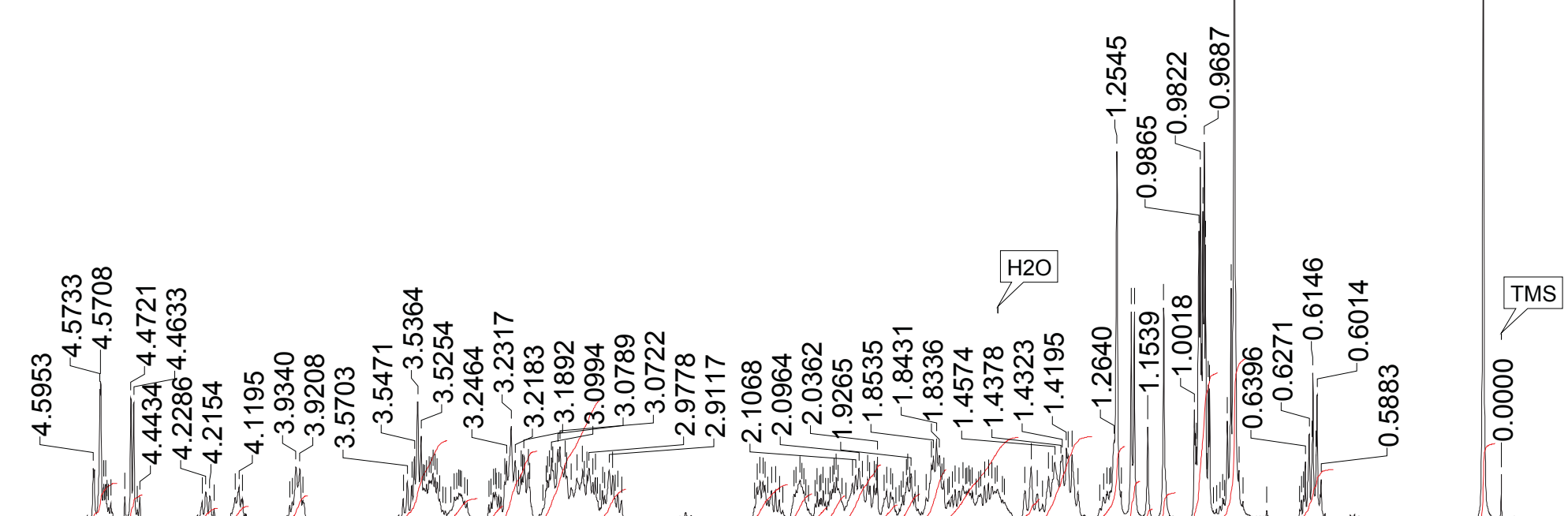




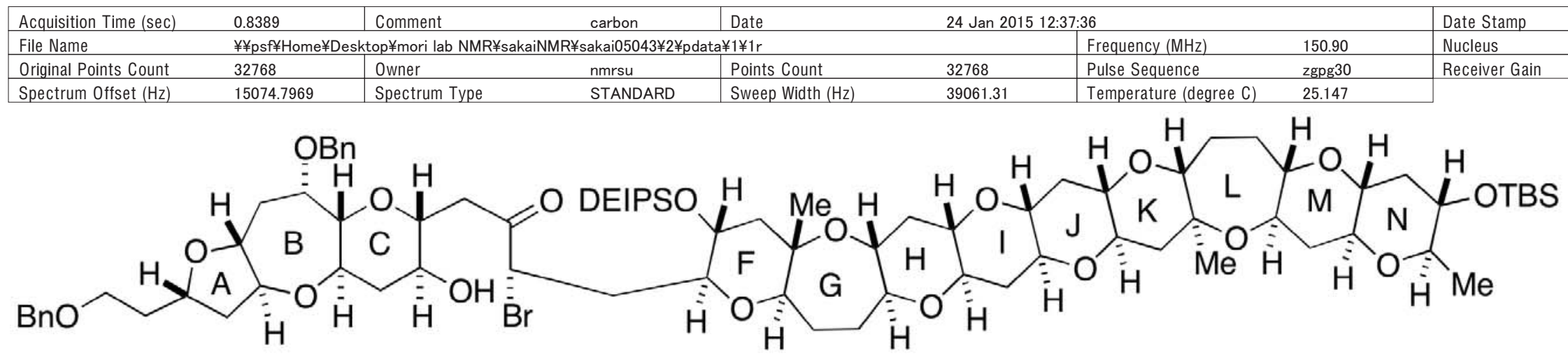

S15 $(\mathrm{dr}=71: 29)$

${ }^{13} \mathrm{C} \mathrm{NMR}\left(\mathrm{CDCl}_{3}, 150 \mathrm{MHz}\right)$

$\stackrel{\infty}{0}$

온

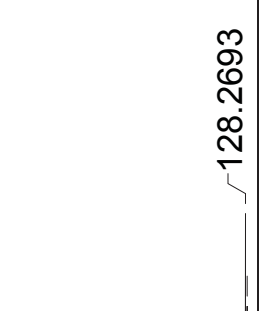

ํํำ

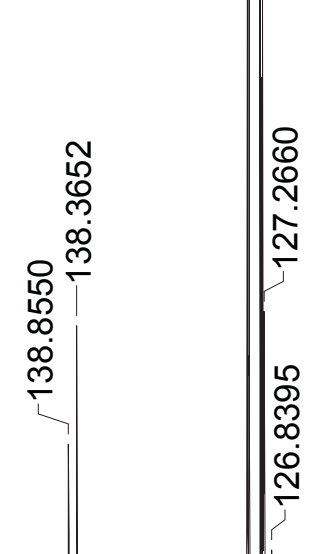

$\mathrm{CDCl3}$

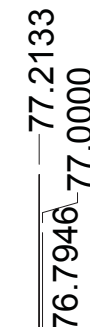

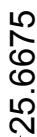

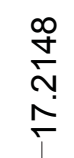

spect
CHLOROFORM-

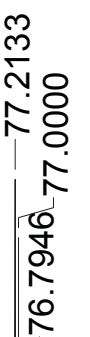

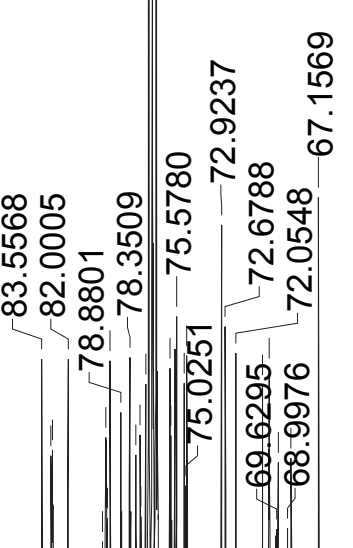

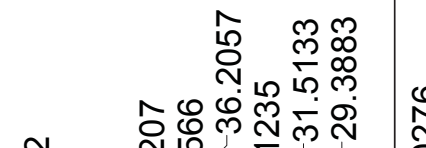

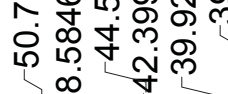

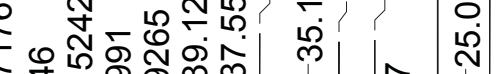




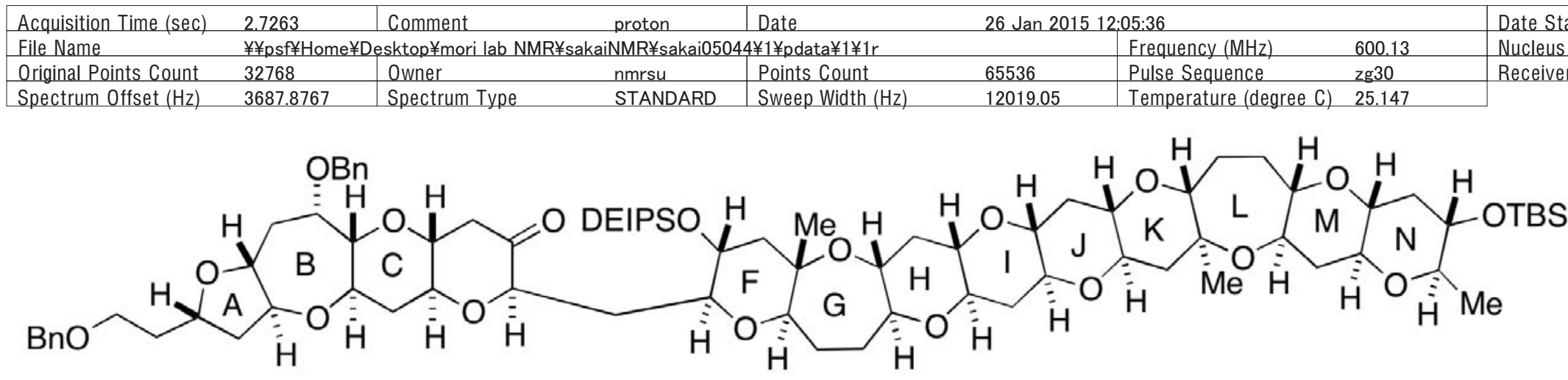

25

$\mathrm{HNMR}\left(\mathrm{CDCl}_{3}, 600 \mathrm{MHz}\right)$
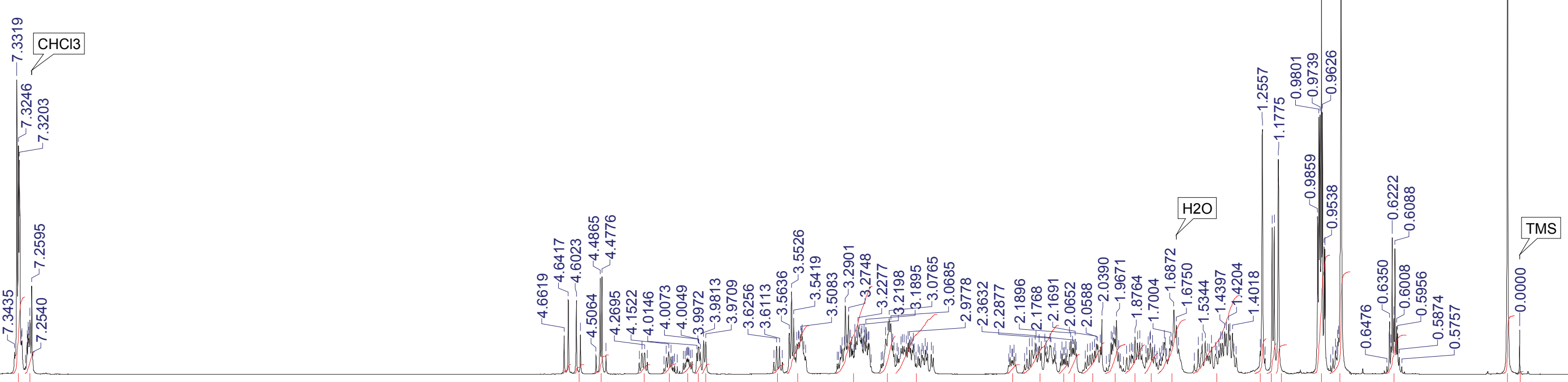

8.222 .33

0.97 $\quad 0.982 .010 .961 .030 .971 .000 .991 .005 .129 .374 .036 .320 .965 .171 .072 .033 .303 .123 .092 .185 .778 .363 .733 .223 .0912 .7010 .914 .116 .190 .19$

\begin{tabular}{|l}
\hline Origin \\
Solvent
\end{tabular} spect 


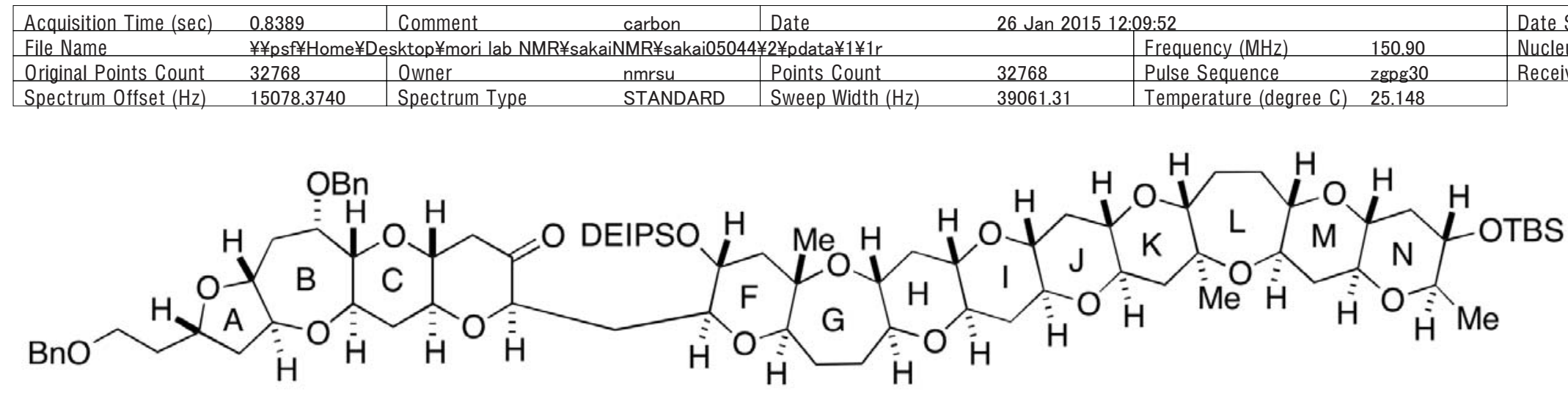

25

${ }^{13} \mathrm{C} \mathrm{NMR}\left(\mathrm{CDCl}_{3}, 150 \mathrm{MHz}\right)$

$\mathrm{CDCl} 3$

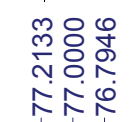

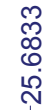

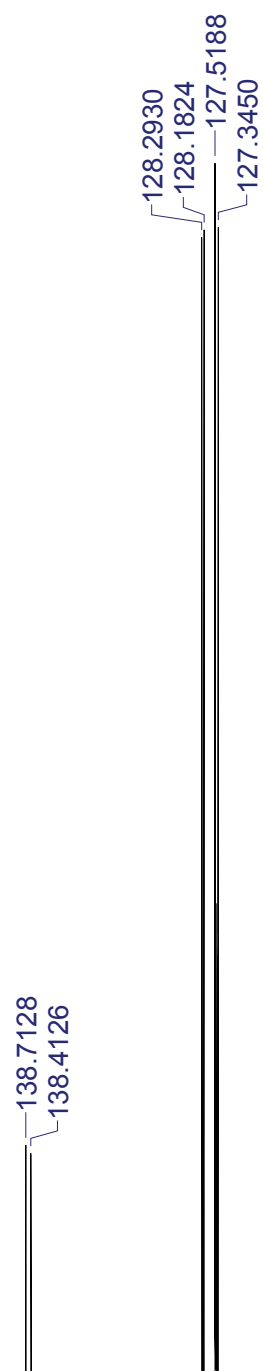

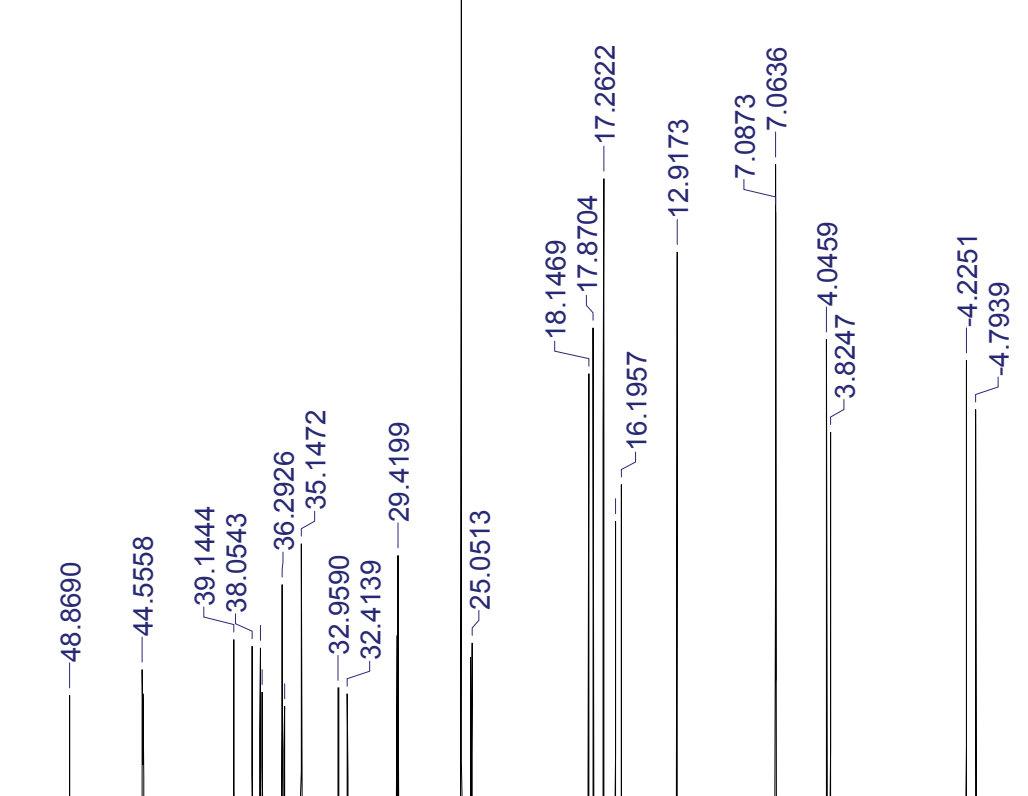






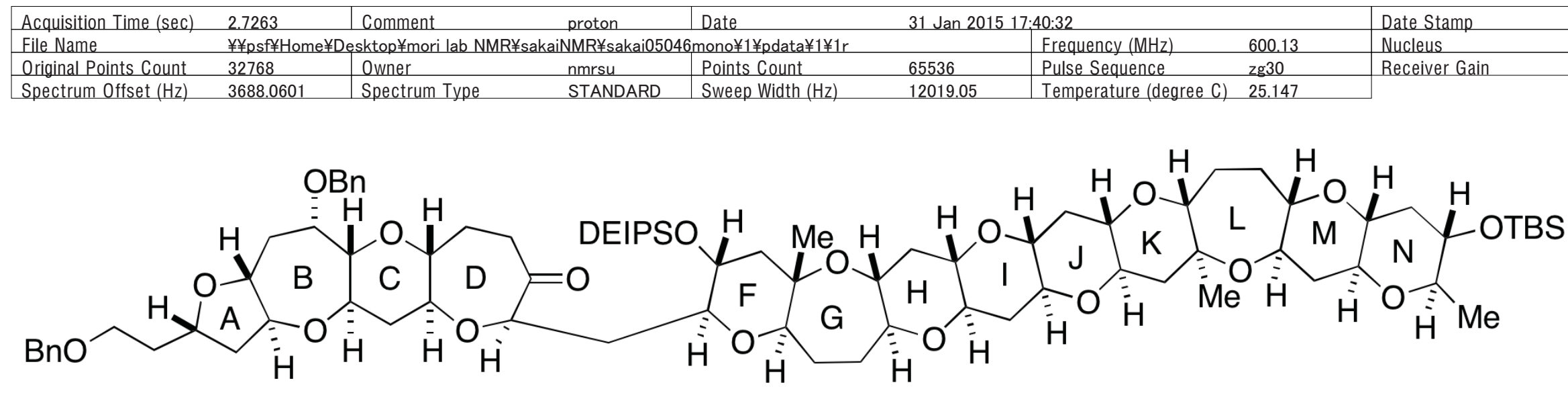

26

${ }^{1} \mathrm{HNMR}\left(\mathrm{CDCl}_{3}, 600 \mathrm{MHz}\right)$

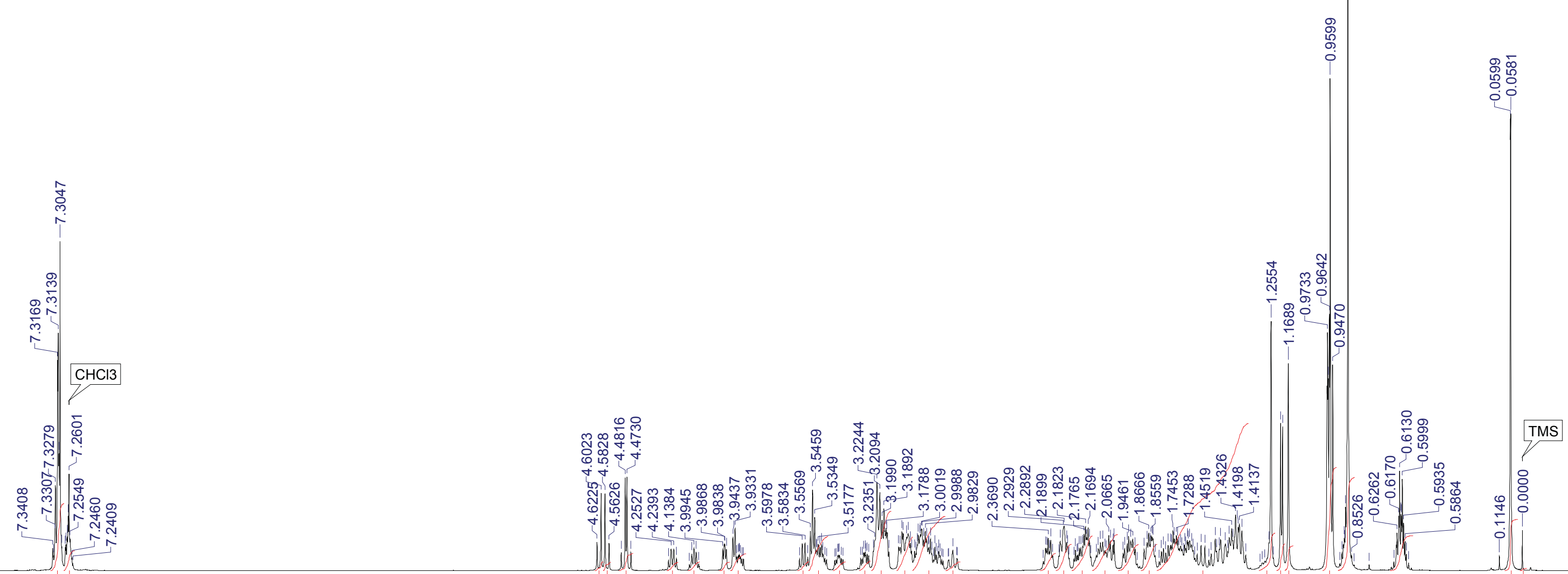

7.962 .36

1.03 0.97 2.02 0.98 1.031 1.00 1.98 1.07 4.07 1.02 1.15 7.05 3.996.431.02 2.05 3.114.124.27 3.103.1317.464.46 3.192.94 12.25 11.06 4.15 


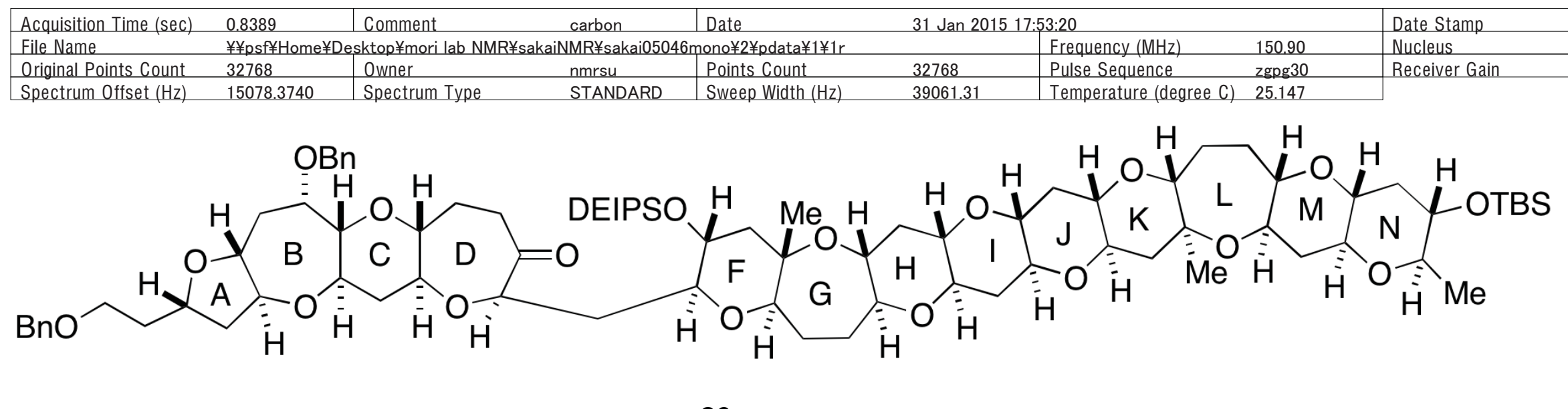

26

${ }^{13} \mathrm{C} \mathrm{NMR}\left(\mathrm{CDCl}_{3}, 150 \mathrm{MHz}\right)$
$13 \mathrm{C} \quad$ Number of Transients 200 \begin{tabular}{l|l|l}
$13 \mathrm{C}$ & Number of Trans \\
193.26 & SW(cyclical) (Hz)
\end{tabular}

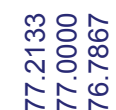

spect
CHLOROFORM-d

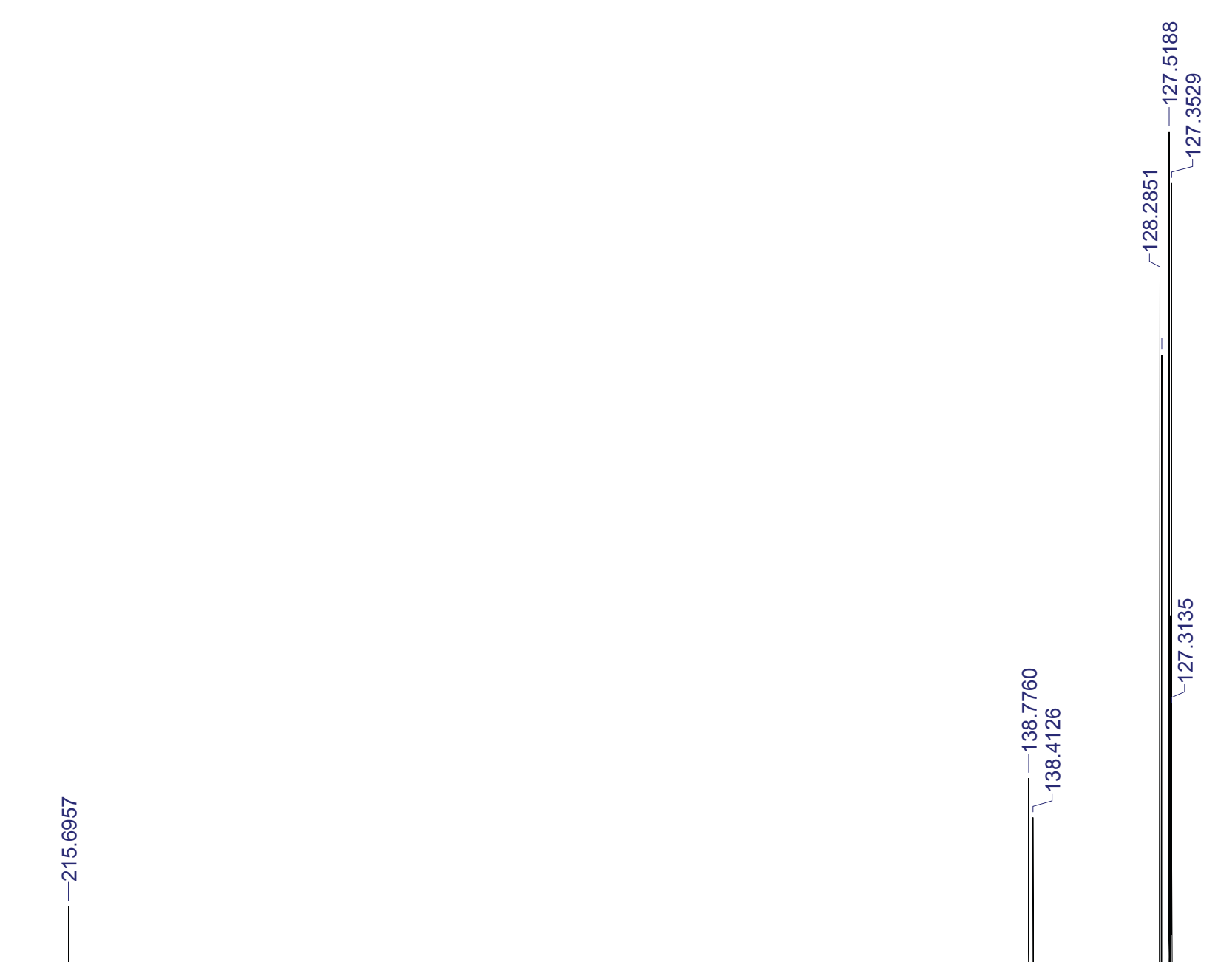




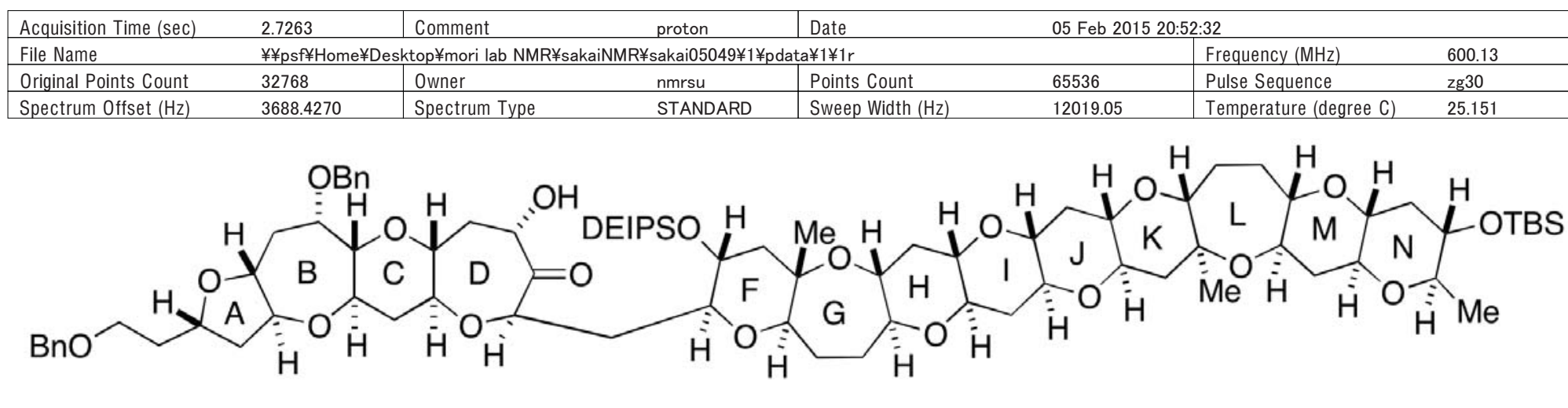

${ }^{1} \mathrm{H} \mathrm{NMR}\left(\mathrm{CDCl}_{3}, 600 \mathrm{MHz}\right)$
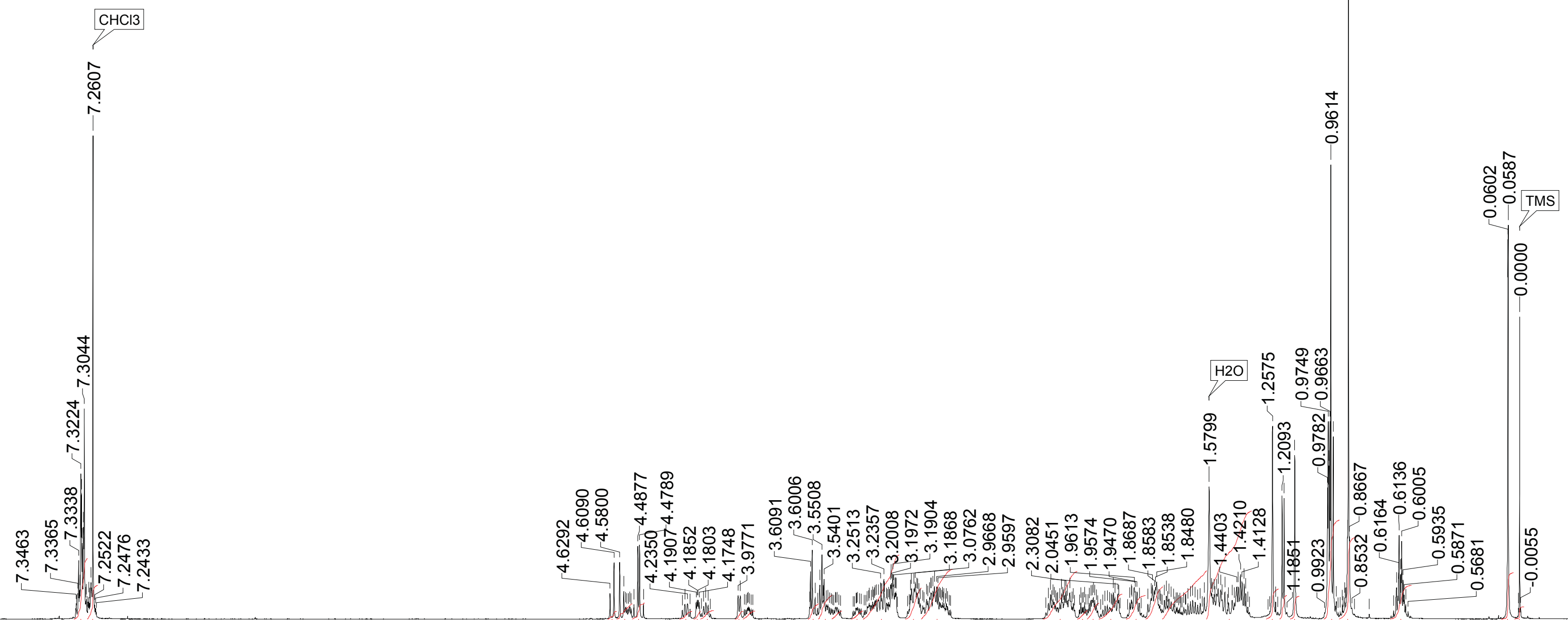

7.654 .24

$0.961 .932 .000 .970 .921 .040 .951 .001 .923 .021 .001 .008 .063 .916 .27 \quad 5.981 .052 .003 .132 .984 .066 .0913 .613 .792 .982 .8812 .4710 .354 .26$

5.841 .54 

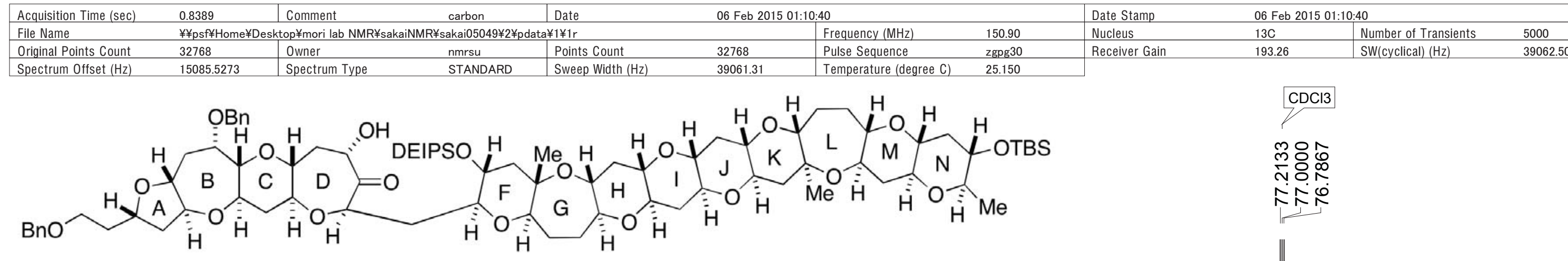

\begin{tabular}{|l|l|}
\hline Origin \\
\hline Solvent
\end{tabular} spect
CHLOROFORM-d

${ }^{13} \mathrm{C} \mathrm{NMR}\left(\mathrm{CDCl}_{3}, 150 \mathrm{MHz}\right)$

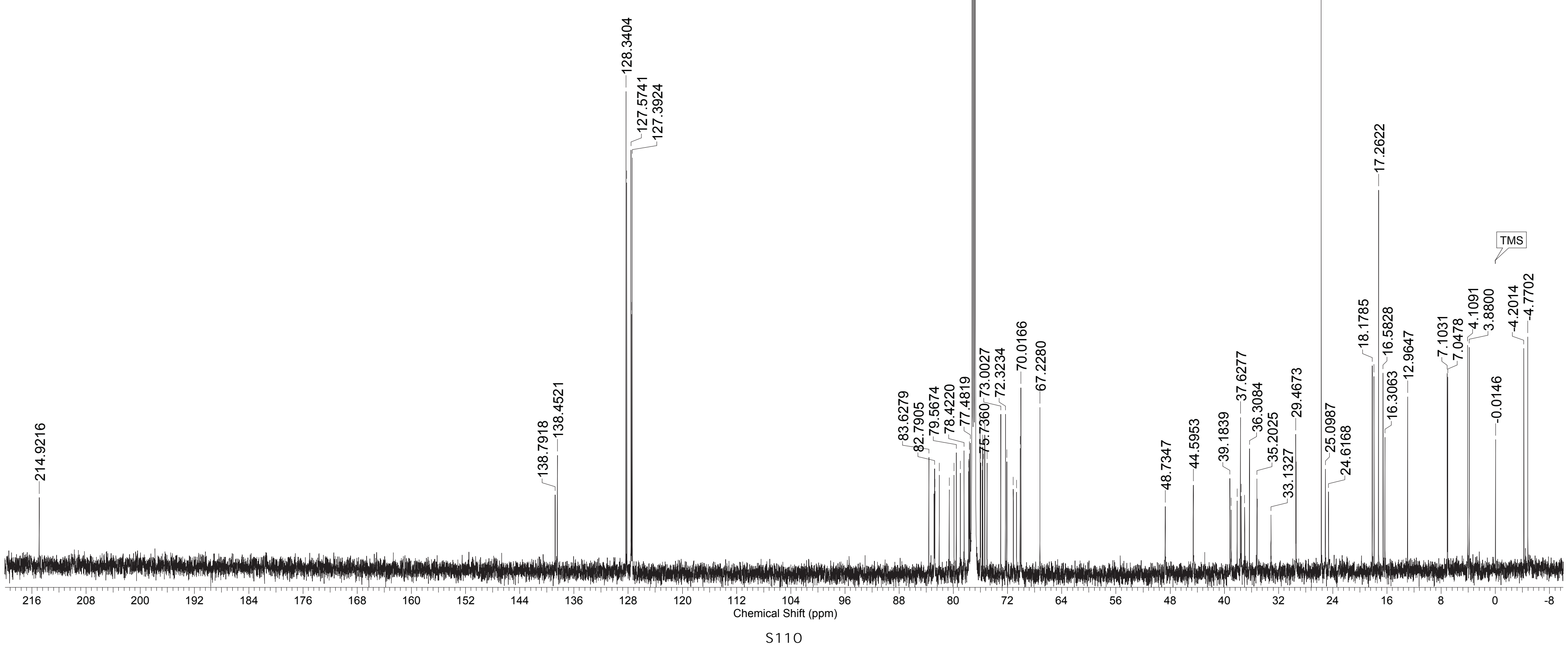




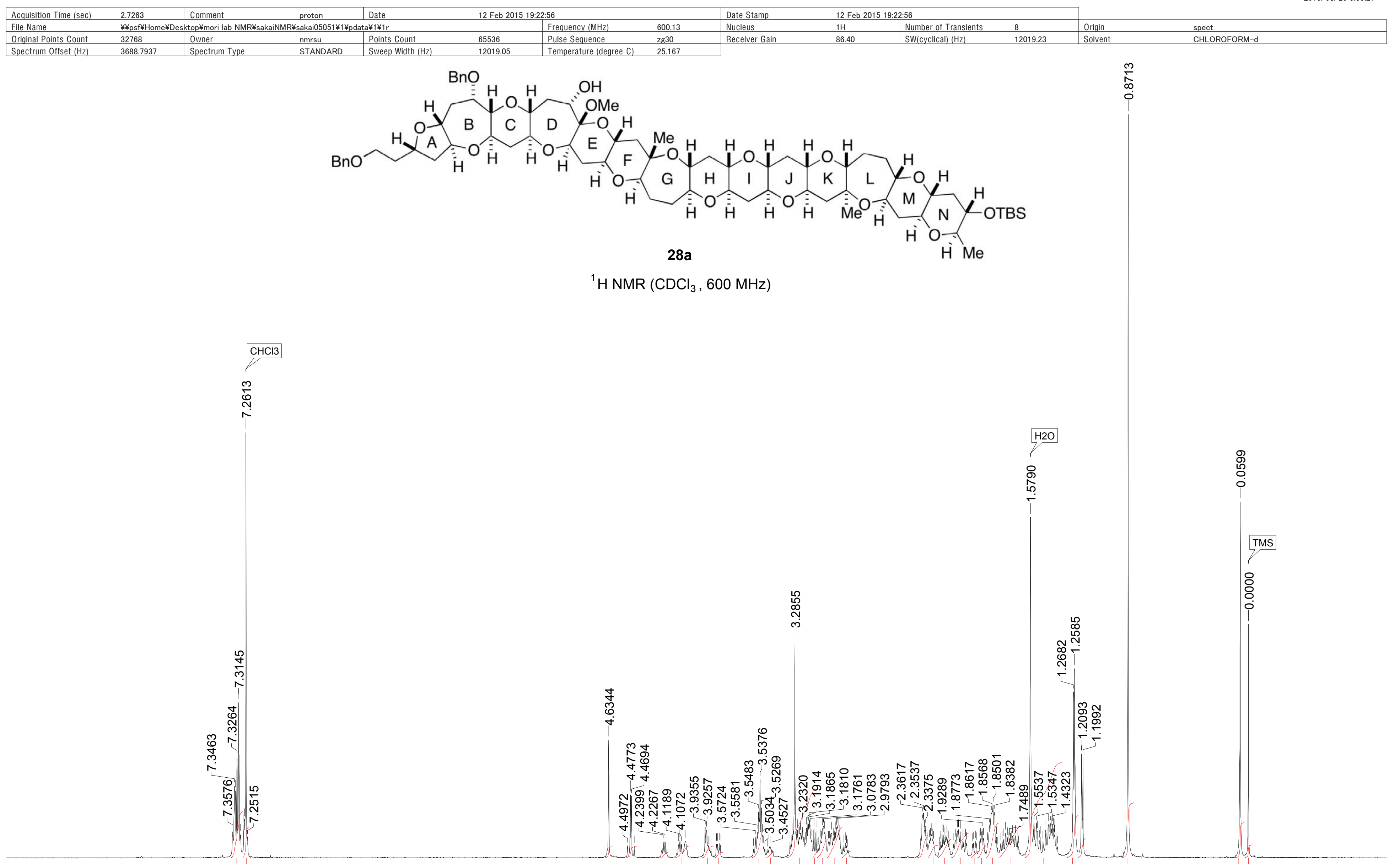




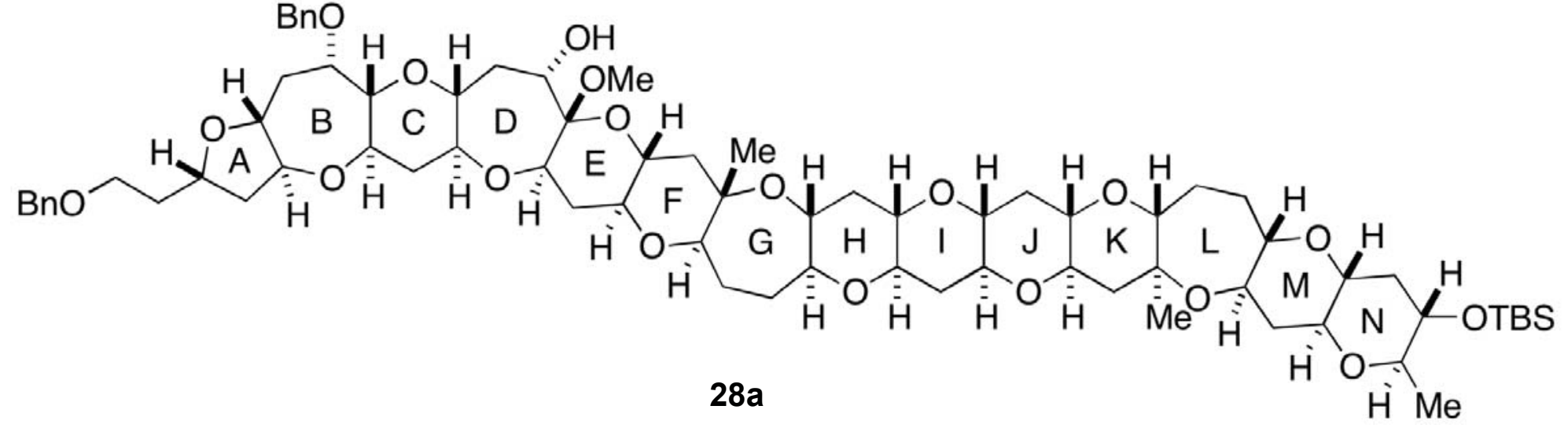

${ }^{13} \mathrm{C} \mathrm{NMR}\left(\mathrm{CDCl}_{3}, 150 \mathrm{MHz}\right)$ 


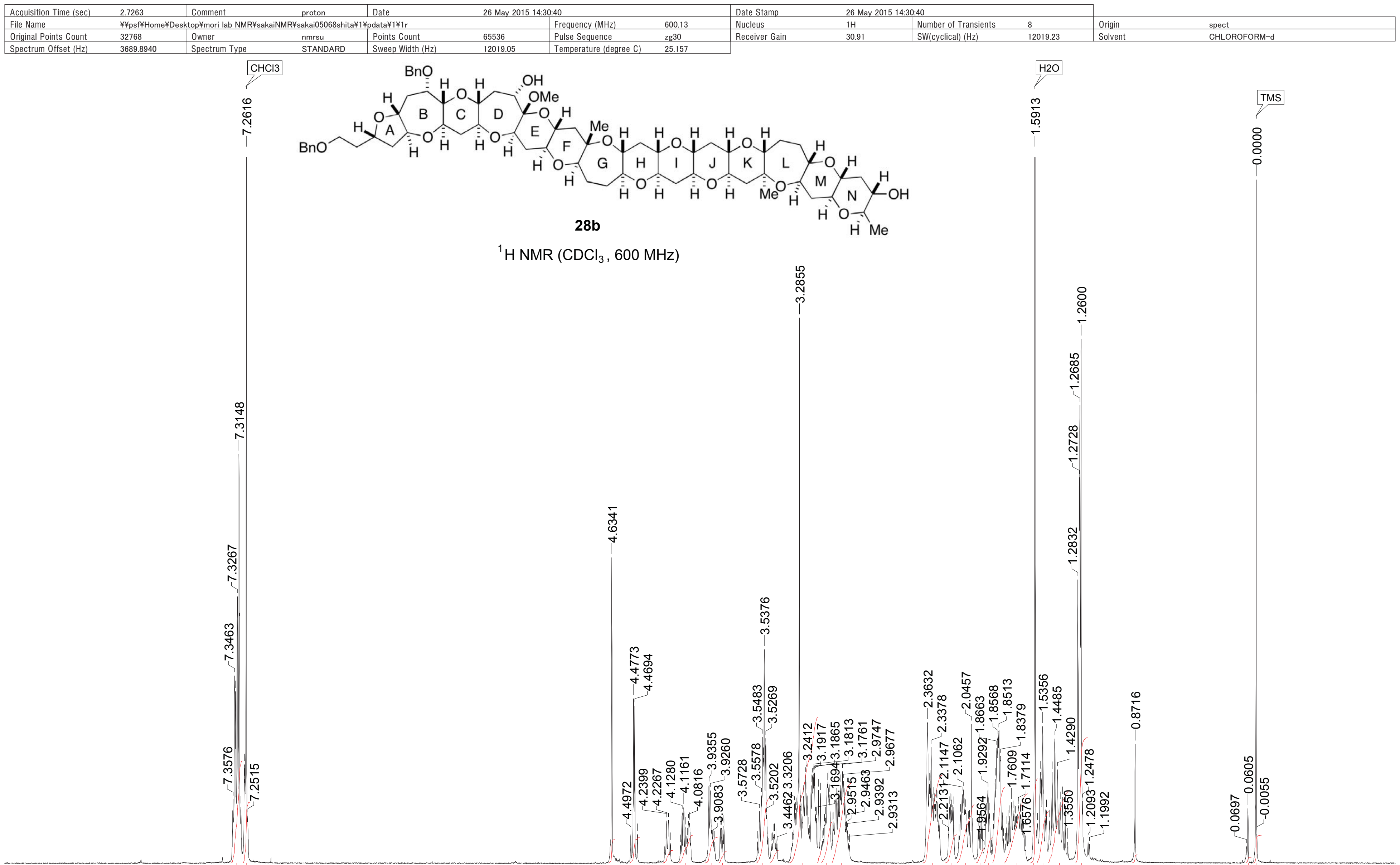

8.054 .81

$1.892 .03 \quad 1.002 .192 .030 .905 .121 .0011 .460 .993 .216 .30 \quad 6.912 .244 .591 .172 .065 .965 .456 .684 .065 .729 .90 \quad 0.66$ 


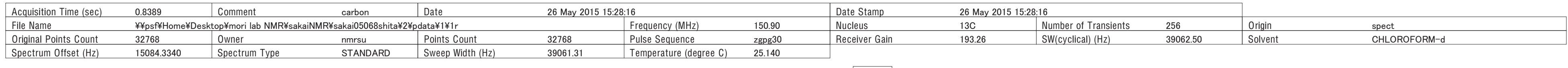

$\mathrm{CDCl3}$

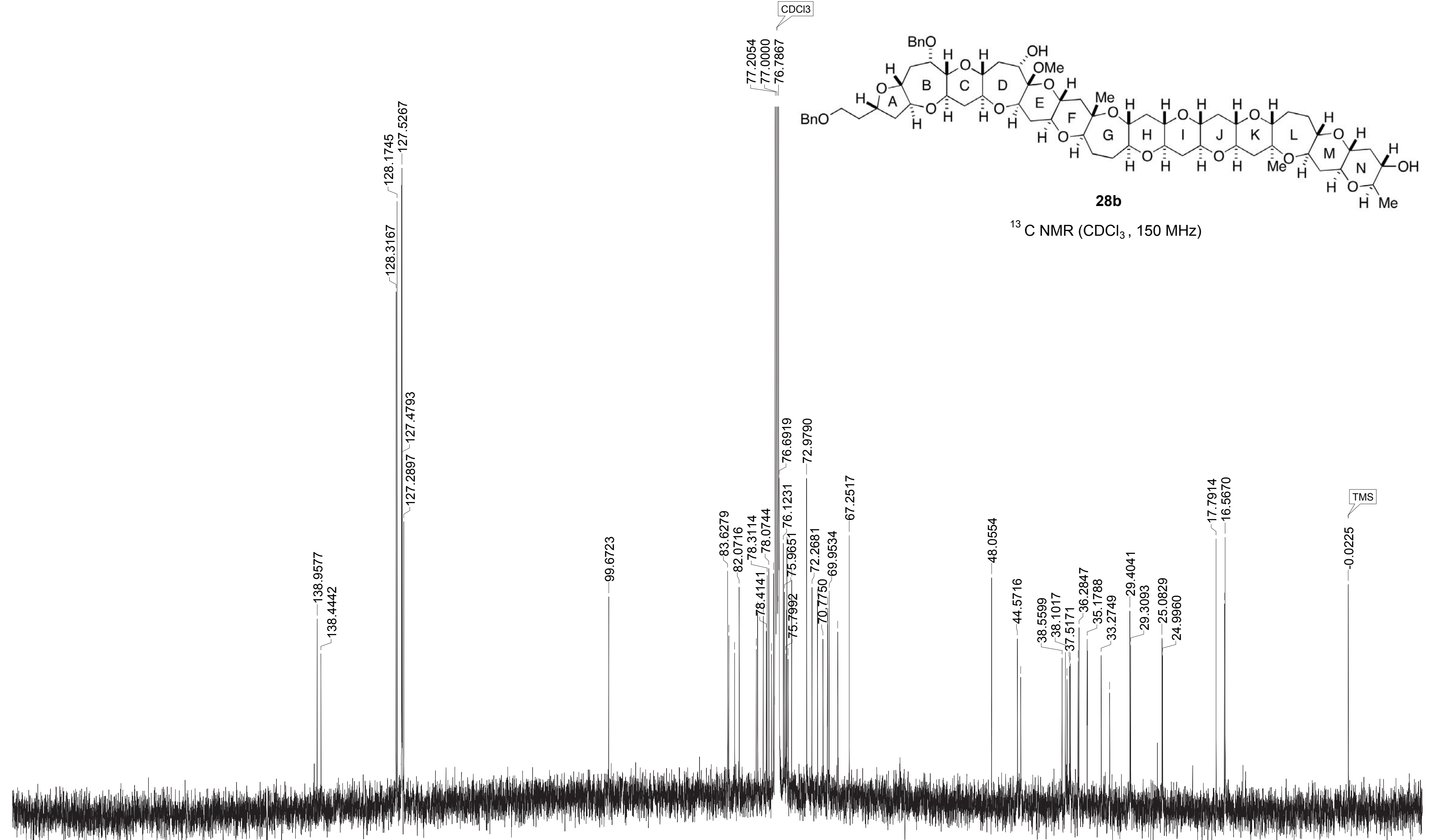




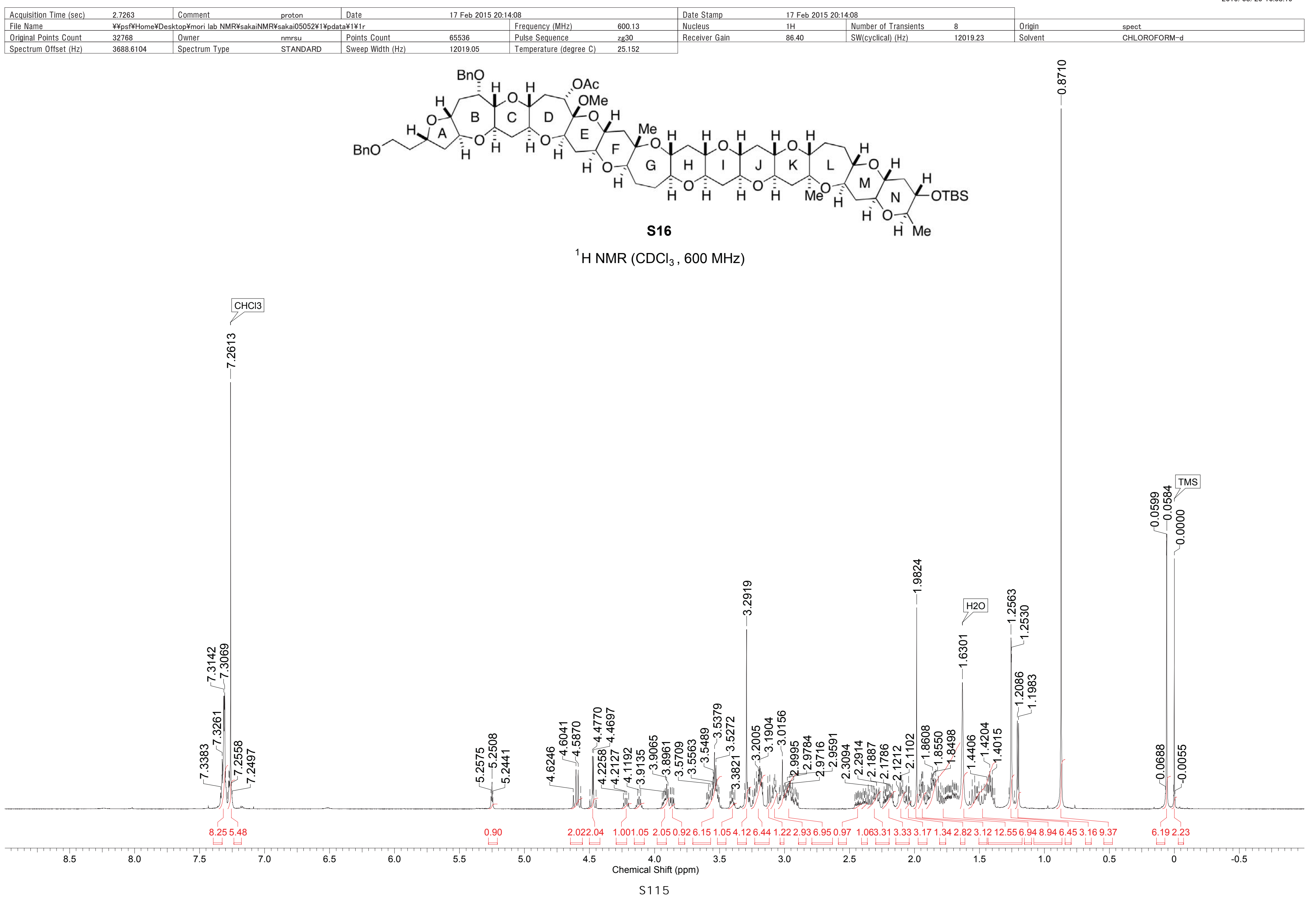




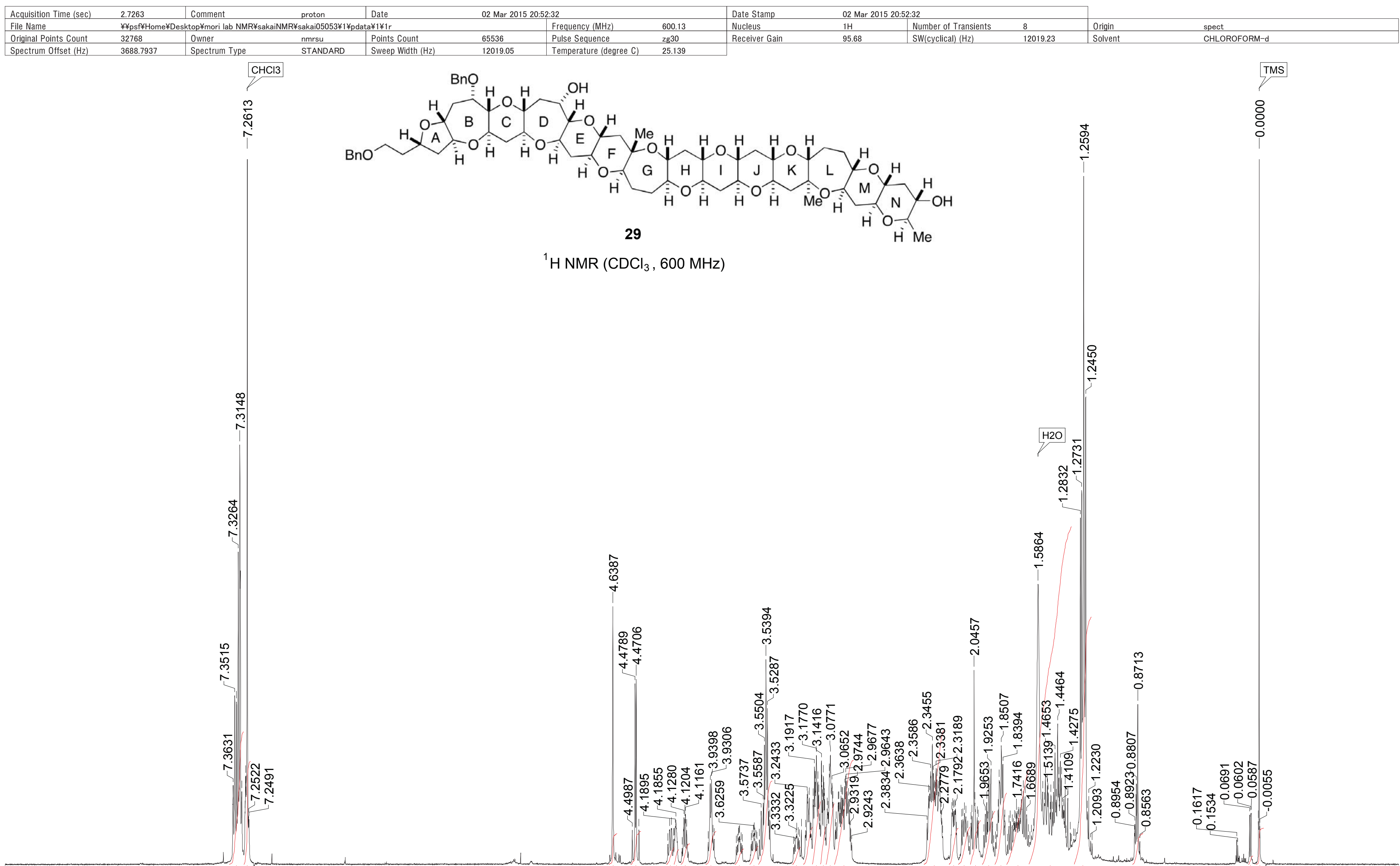




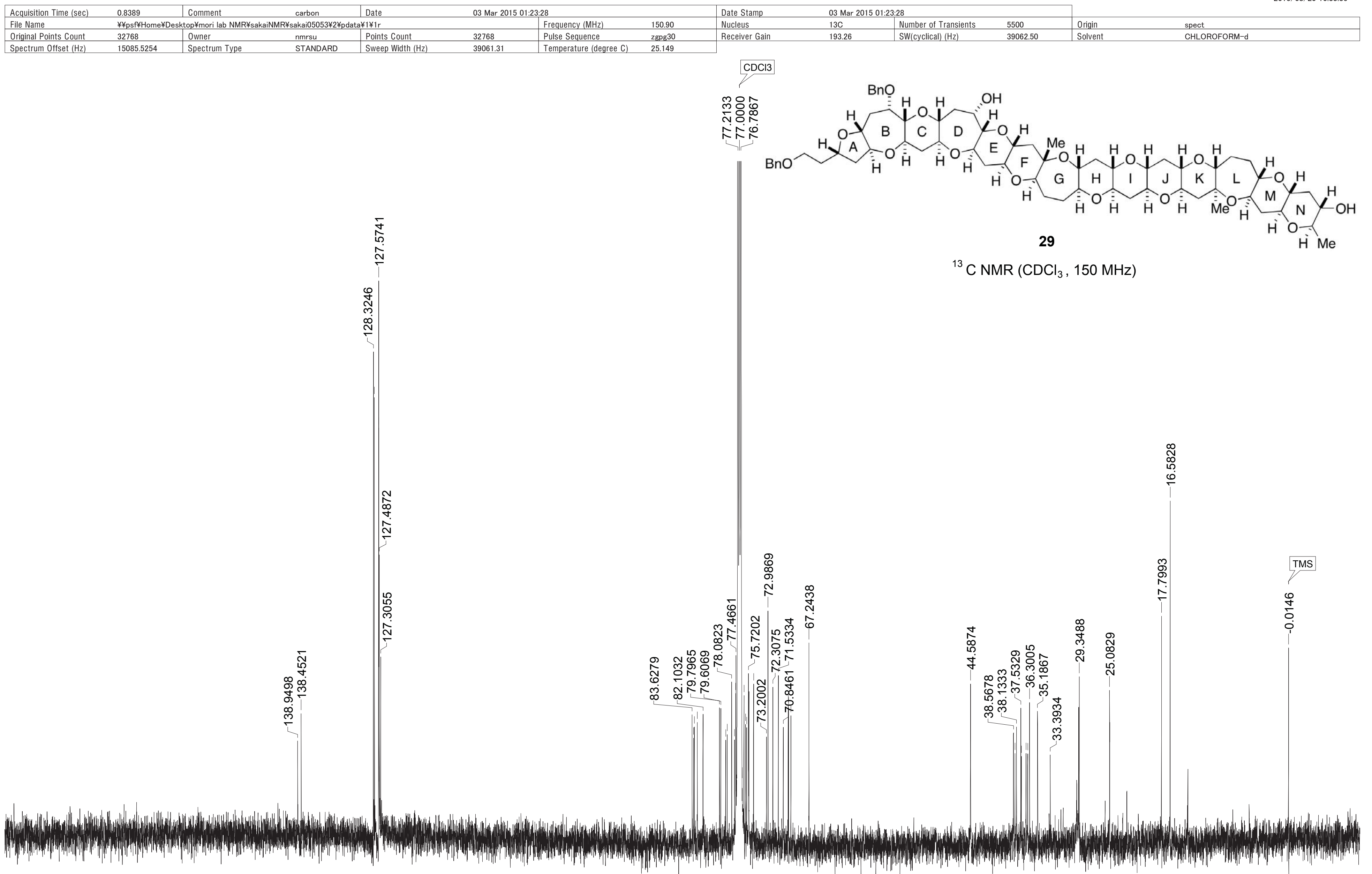




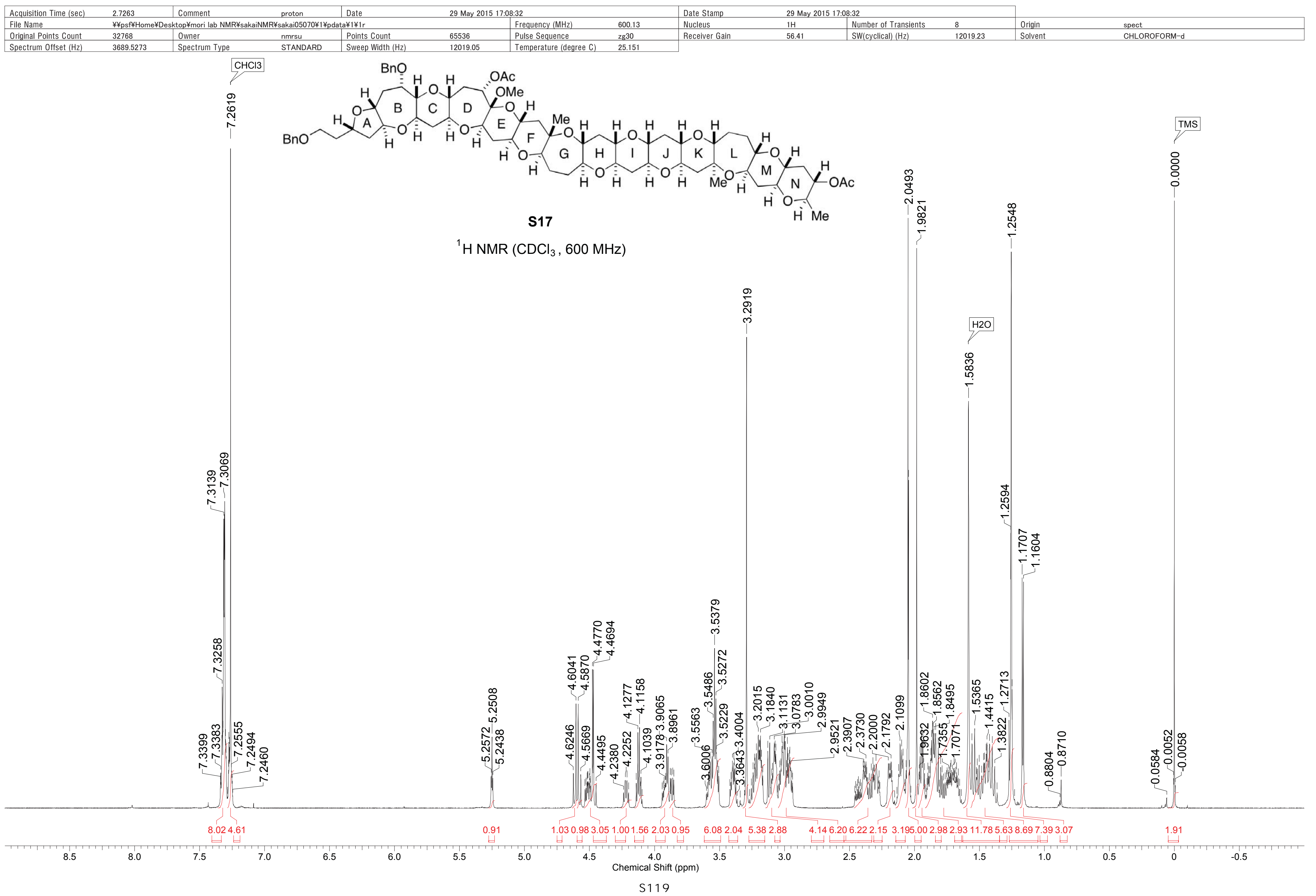




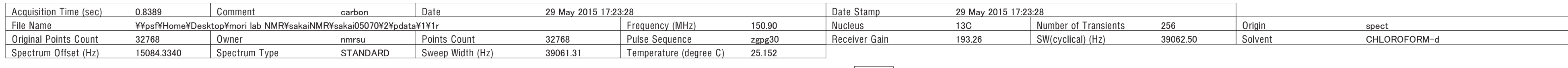

$\mathrm{CDCl3}$

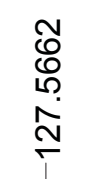

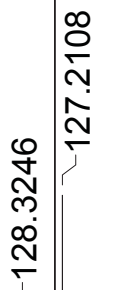

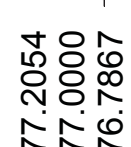

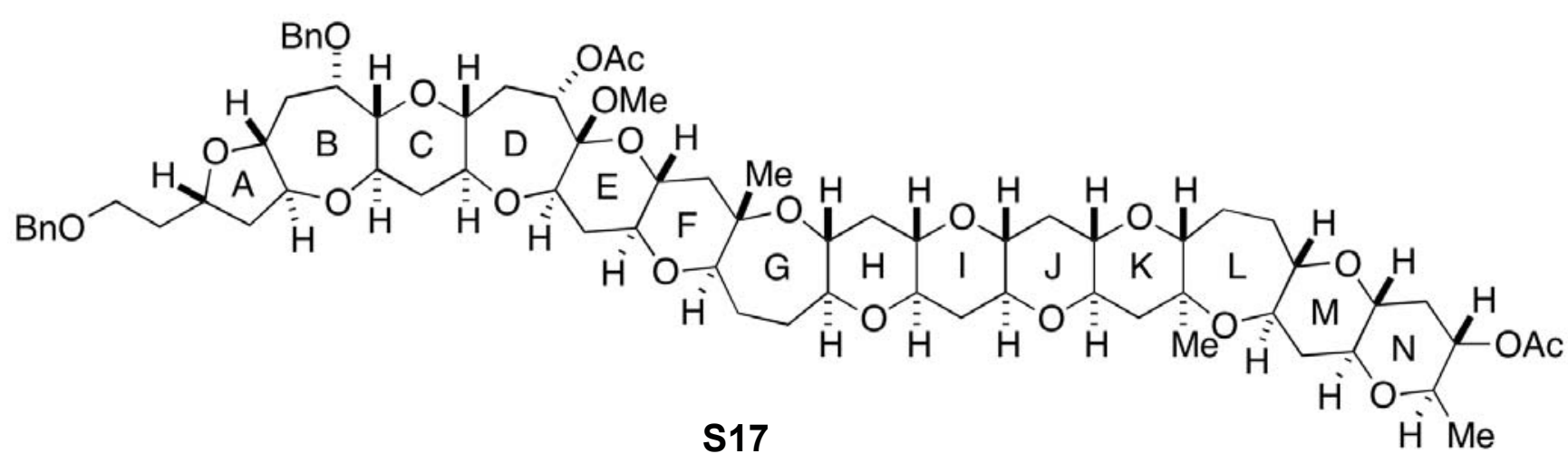

${ }^{13} \mathrm{C} \mathrm{NMR}\left(\mathrm{CDCl}_{3}, 150 \mathrm{MHz}\right)$



:

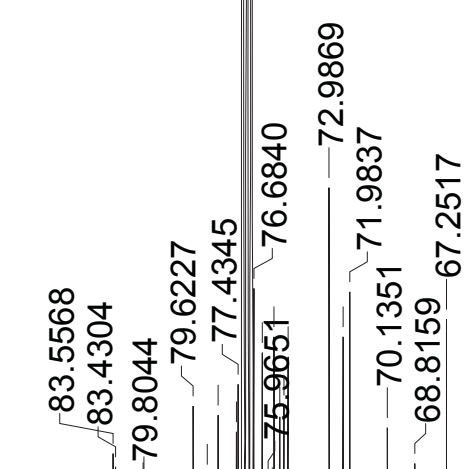


This report was created by ACD/NMR Processor Academic Edition. For more information go to www.acdlabs.com/nmrproc/

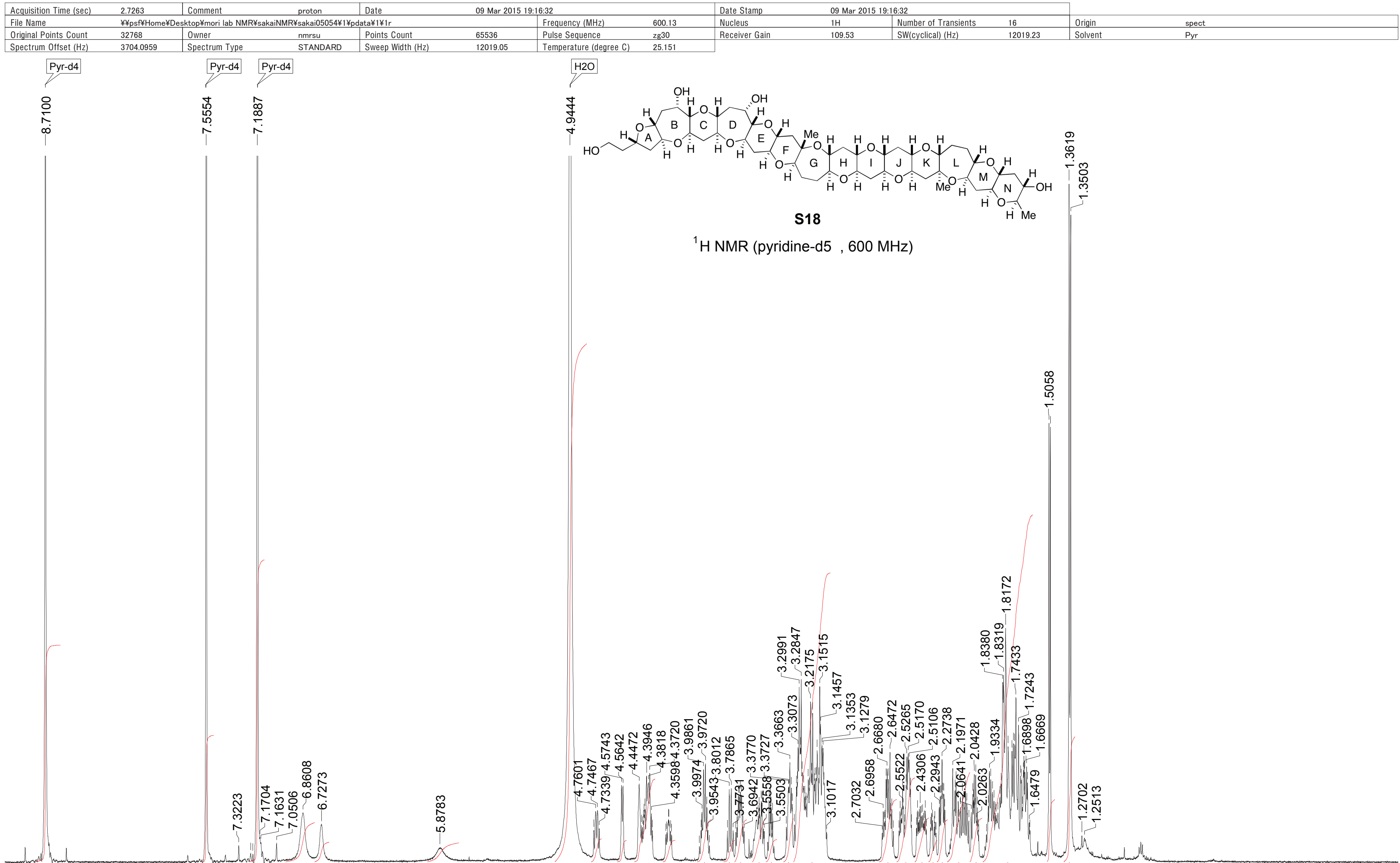


This report was created by ACD/NMR Processor Academic Edition. For more information go to www.acdlabs.com/nmrproc/

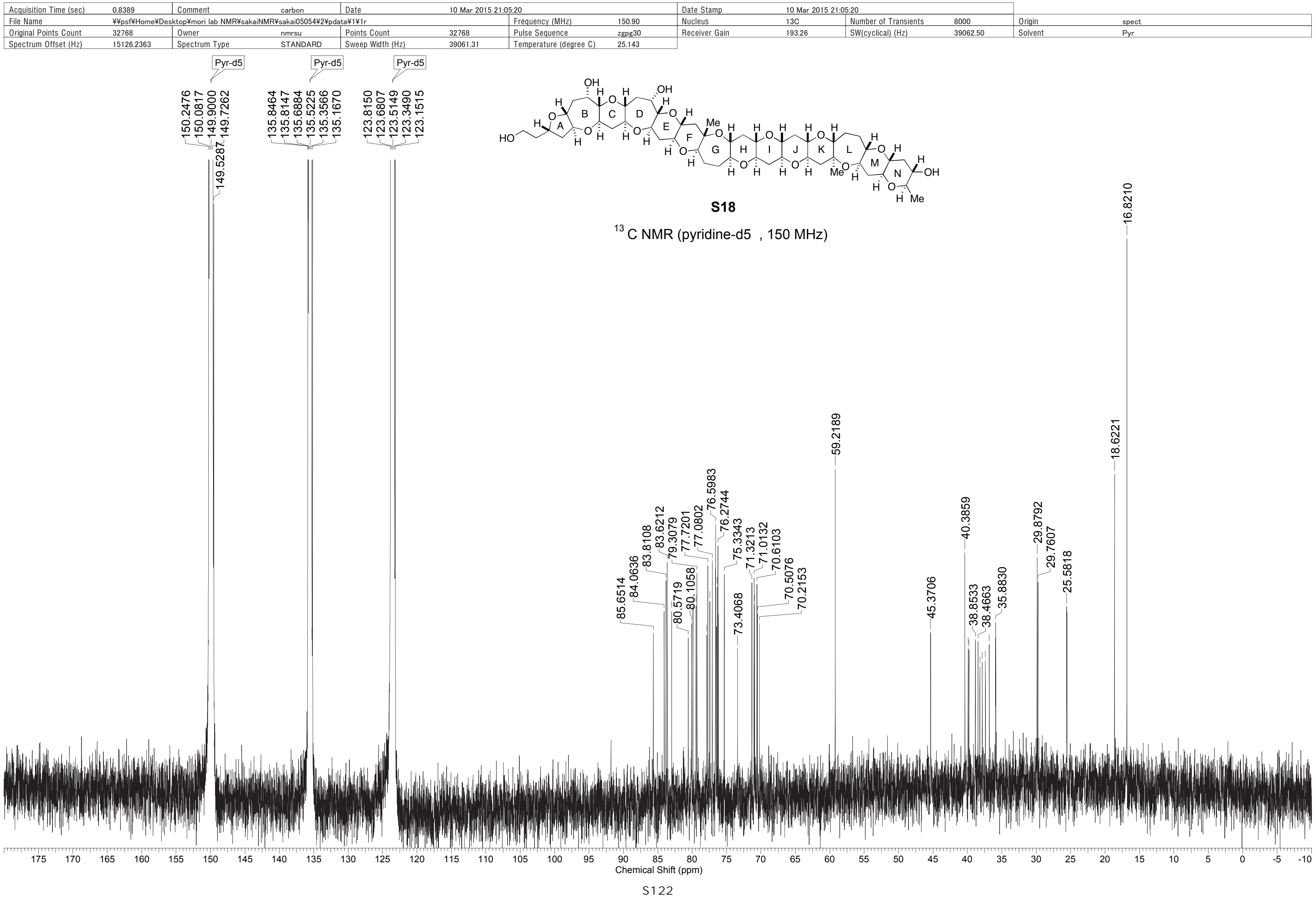




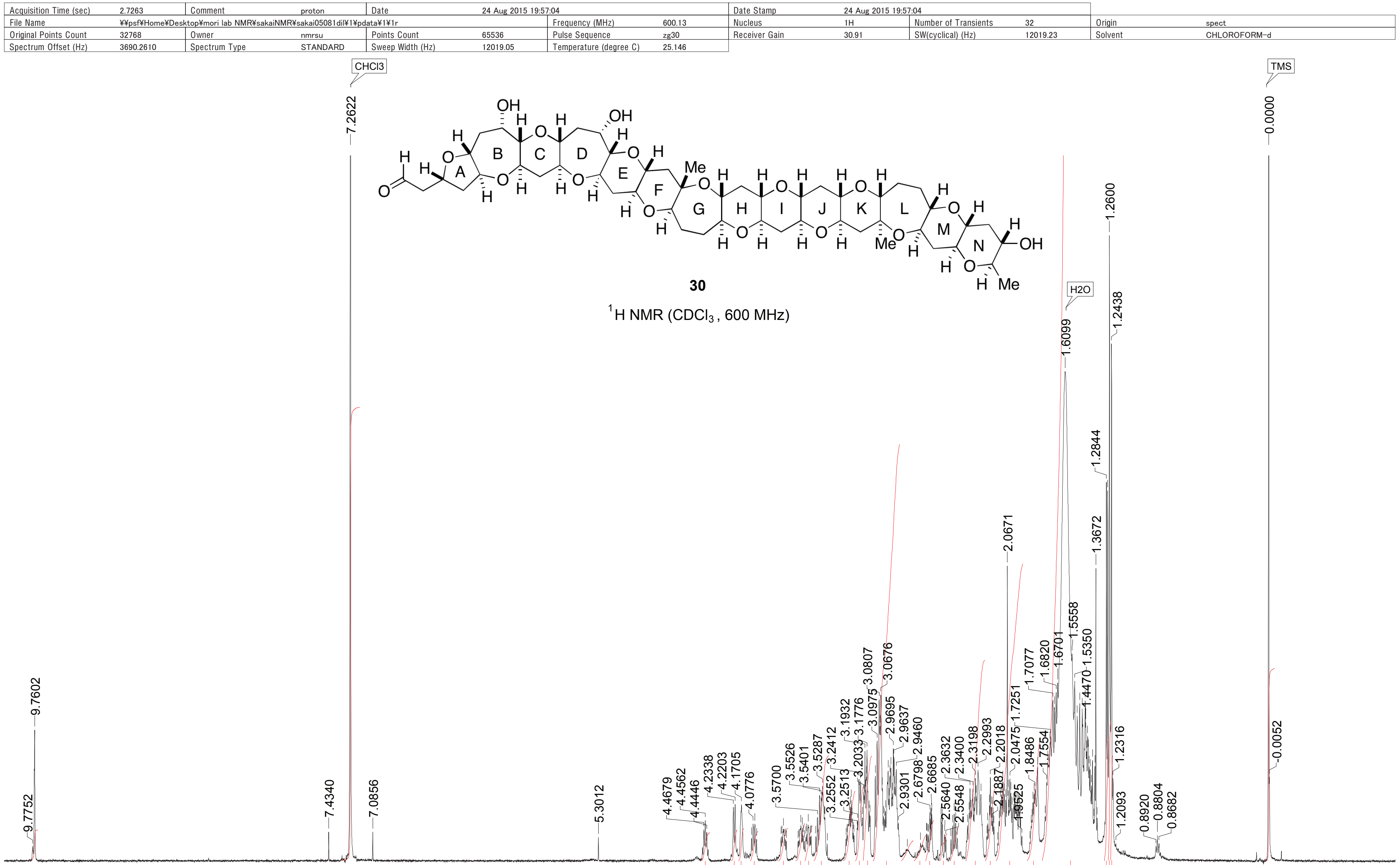




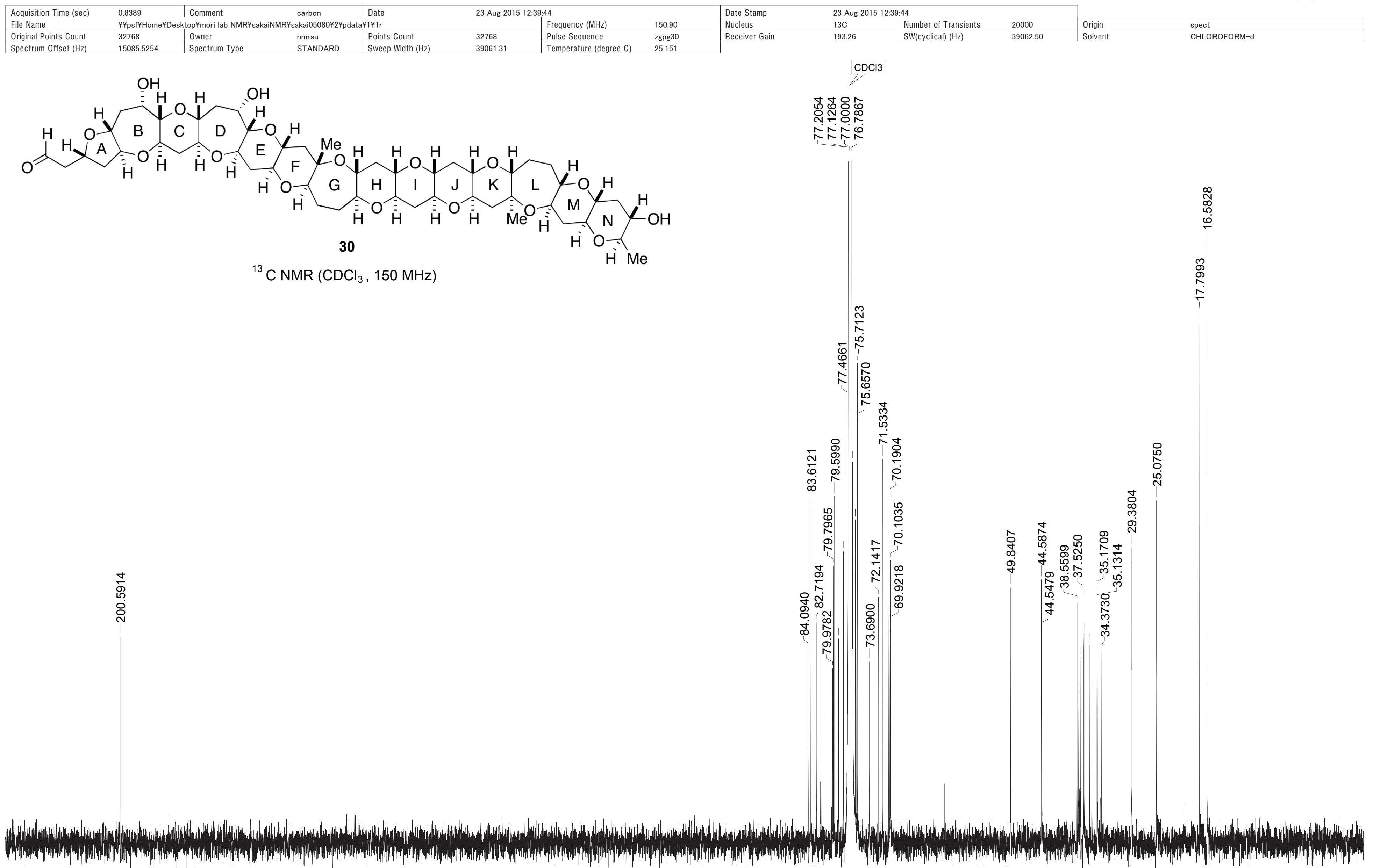


This report was created by ACD/NMR Processor Academic Edition. For more information go to www.acdlabs.com/nmrproc/






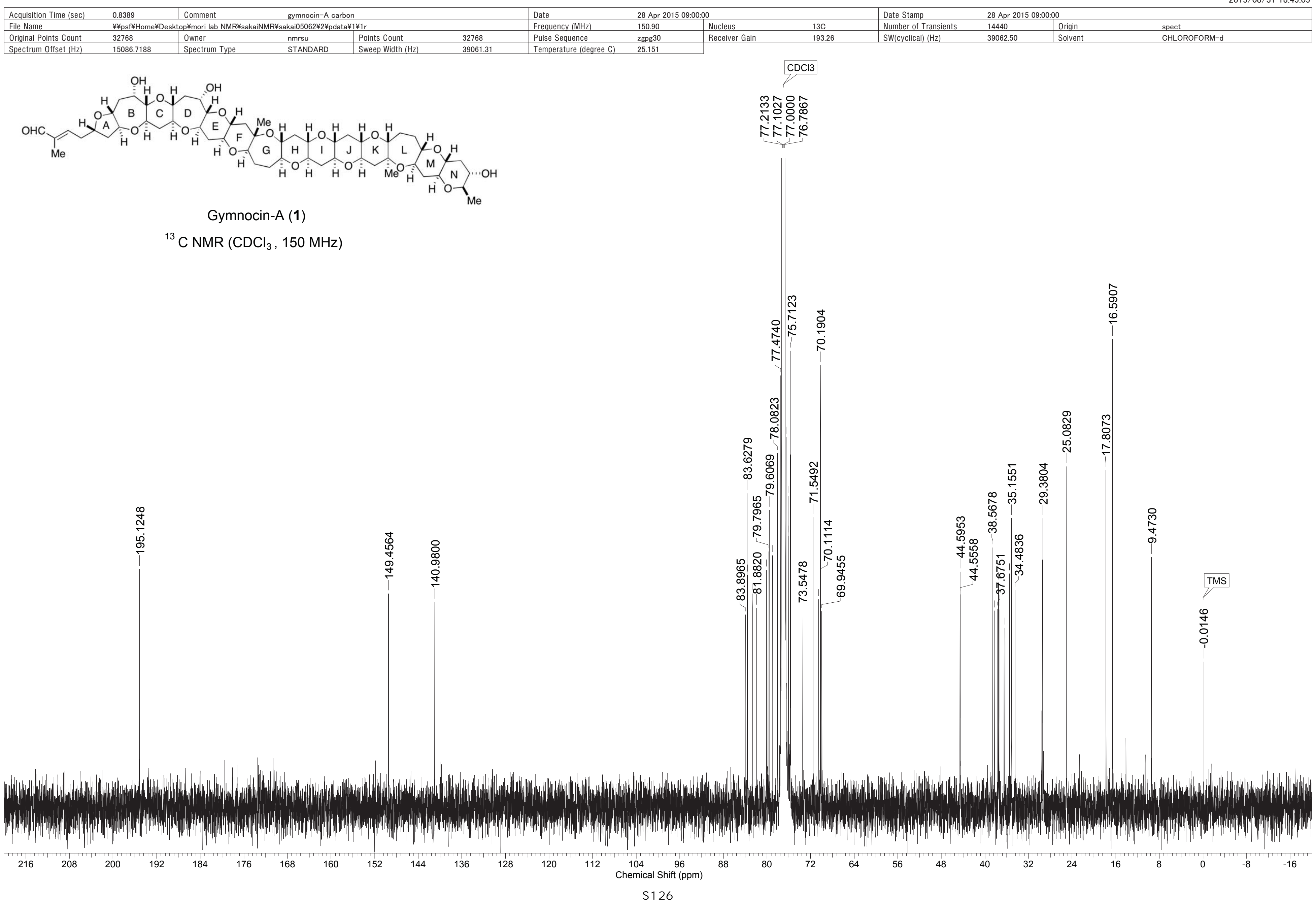









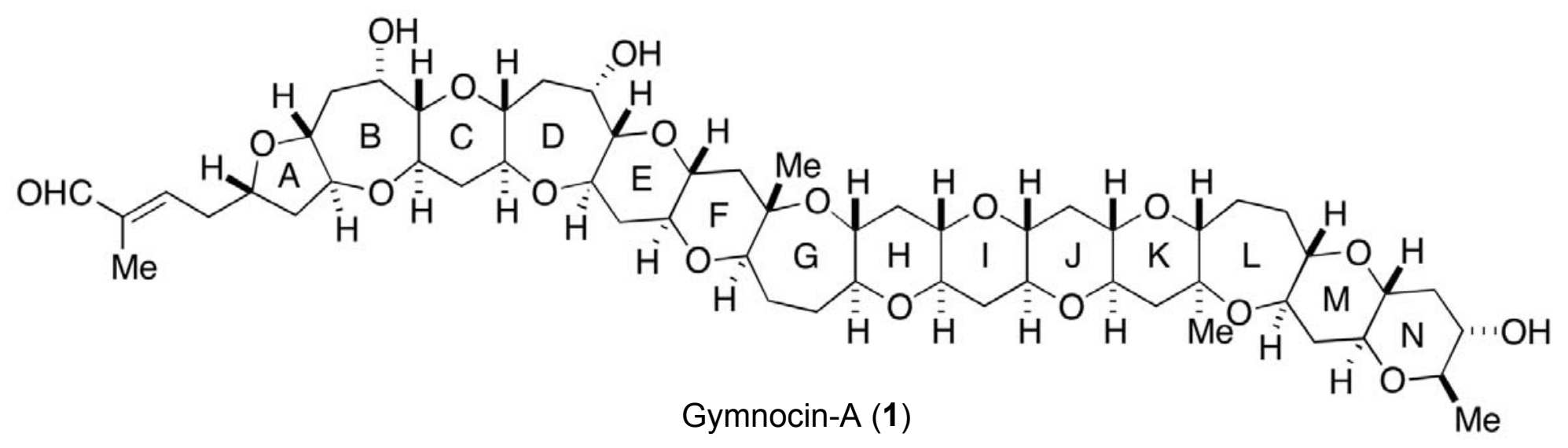

Synthetic

$\left(\mathrm{CDCl}_{3}, 150 \mathrm{MHz}\right)$

\section{Natural}

(Satake, M. et al. Tetrahedron Lett. 2002, 43, 5829)

$1\left(\mathrm{CDCl}_{3}, 150 \mathrm{MHz}\right)$

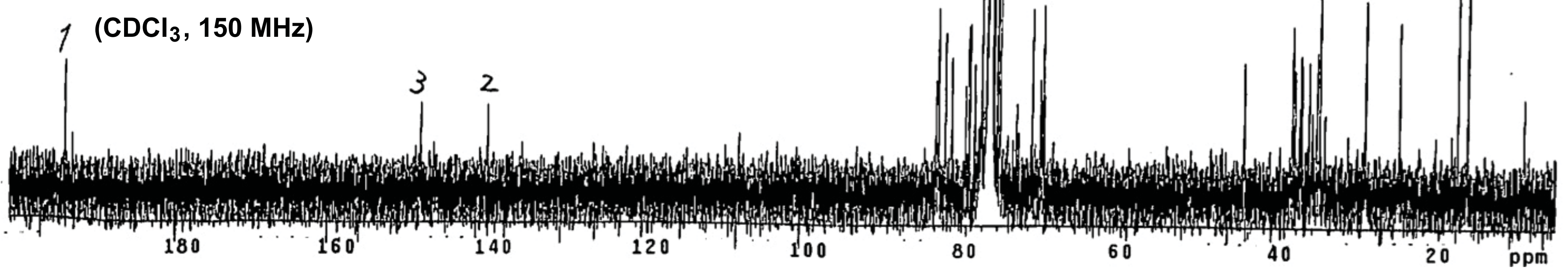

